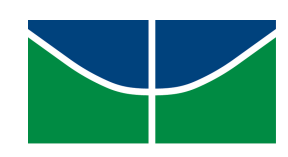

\author{
Universidade de Brasília \\ Instituto de Ciências Exatas \\ Departamento de Matemática
}

\title{
O Operador DE RUELlE EM ESPAÇOS DE ESTADOS COMPACTOS
}

\author{
por
}

Eduardo Antonio da Silva

Orientador: Leandro Martins Cioletti

Durante o desenvolvimento destre trabalho o autor recebeu auxílio financeiro do CNPq

Brasilia-DF

26 de agosto de 2016 



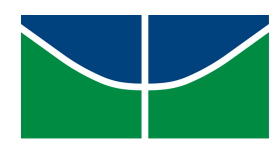

\author{
Universidade de Brasília \\ Instituto de Ciências Exatas \\ Departamento de Matemática
}

\title{
O OpERADOR DE RUELLE EM ESPAÇOS DE ESTADOS COMPACTOS
}

\author{
por
}

Eduardo Antonio da Silva

Orientador: Leandro Martins Cioletti

Tese apresentada ao Programa de Pós-Graduação em Matemática da Universidade de Brasília como requisito parcial à obtenção do título de Doutor em Matemática.

Brasilia-DF

26 de agosto de 2016 


\section{Comissão Julgadora:}

Leandro Martins Cioletti

UnB

\begin{tabular}{c} 
Artur Oscar Lopes \\
UFRGS \\
\hline
\end{tabular}

Ricardo Parreira da Silva

UnB
Carlos Tomei

PUC-Rio

Ricardo Ruviaro

$\mathrm{UnB}$ 


\section{AGRADECIMENTOS}

Quero agradecer a minha mãe e meu pai, os quais, cada qual à sua maneira, contribuiram pra este momento.

Sou grato ao meu irmão pelos vários momentos que compartilhamos juntos estudando e fazendo matemática.

Não posso deixar de agradecer a minha amada esposa Andréia, por estar ao meu lado nos momentos mais difíceis e nos momentos felizes também. Estar do seu lado deu um novo tom à minha vida.

Por fim gostaria de deixar um agradecimento especial aos professores Carlos Tomei e Leandro Cioletti, que desempenharam um papel fundamental em minha trajetória até aqui. A vocês, a minha profunda gratidão. 


\section{RESUMO}

Recentemente o Teorema de Perron Fröbenius foi provado para potenciais Hölder definidos no espaço simbólico $\Omega=M^{\mathbb{N}}$, onde o alfabeto $M$ é um espaço métrico compacto qualquer. Nesta tese estendemos este teorema para potenciais definidos no espaço de Walters $W(\Omega)$, em alfabetos similares. No resultado acima seguimos de perto o trabalho original [4], as diferenças principais da demonstração moram em argumentos a respeito do suporte da medida a priori.

Descrevemos em detalhes um procedimento abstrato para obter a analiticidade no sentido de Fréchet do operador de Ruelle sob condições bastantes gerais e usamos isso para obter a dependência analítica desse operador em ambos os espaços $C^{\gamma}(\Omega)$ e $W(\Omega)$. Este resultado é importante pois está intrinsecamente relacionado com a analiticidade da pressão. Para este fim foi necessário estabelecer em $W(\Omega)$ uma estrutura de álgebra de Banach, bem como sua invariância pelo operador de Ruelle. Esses resultados são novos tanto no contexto de espaço de estados compactos quanto no contexto de espaços de estados finitos.

A regularidade do funcional pressão é de grande interesse pois tem relação indissociável com transição de fase. Neste trabalho estabelecemos a analiticidade do funcional pressão no espaço dos potenciais Hölder. Nesta demonstração é usado de modo fundamental que para potenciais Hölder o operador de Ruelle possui a propriedade do buraco espectral.

Um decaimento exponencial de correlações é provado quando o operador de Ruelle tem a propriedade do buraco espectral.

Uma nova (e natural) família de potenciais na classe de Walters (em um alfabeto finito derivada do modelo de Ising) não possuindo um decaimento exponencial de correlações é apresentada. A idéia por trás desse resultado é usar o dicionário estabelecido em [9] entre o formalismo termodinâmico e o modelo de Ising para usar fortemente as desigualdades de GriffithsKelly-Sherman, já estabelecidas no contexto da mecânica estatística para mostrar um exemplo, também conhecido pela comunidade da mecânica estatística, com decaimento superpolinomial de correlações. Devido à ausência de decaimento de exponencial de correlações temos por conseguinte a ausência do buraco espectral para o operador de Ruelle.

A sistemática acima também fornece uma nova abordagem para a obtenção de exemplo de decaimento de correlações, que não pode ser recuperado pelos resultados apresentados em [42] a respeito de decaimentos de correlações subexponenciais nem do trabalho de Gouëzel em [25], que generaliza o anterior.

Palavras chave: Formalismo Termodinâmico, Operador de Ruelle, lattice uni-dimensional, Analiticidade da pressão, buraco espectral. 


\section{ABSTRACT}

Recently the Ruelle-Perron-Fröbenius theorem was proved for Hölder potentials defined on the symbolic space $\Omega=M^{\mathbb{N}}$, where (the alphabet) $M$ is any compact metric space. In this thesis, we extend this theorem to the Walters space $W(\Omega)$, in similar general alphabets. In the above result we follow closely [48], the main differences of the proof are in the argument respecting the support of the a priori measure.

We also describe in detail an abstract procedure to obtain the Fréchet-analyticity of the Ruelle operator under quite general conditions and we apply this result to prove the analytic dependence of this operator on both Walters and Hölder spaces. This result is important, because is closely related with the regularity of the pressure. To this end it was necessary establish in $W(\Omega)$ a Banach algebra structure, as well as its invariance by the Ruelle operator. These results are new in both contexts, finite or general compact space of states.

The regularity of the pressure functional is of great interest since it is closely related with the phase transition. In this work we establish the analiticity of the pressure functional on the Hölder spaces $C^{\gamma}(\Omega)$. In the proof it is used in a fundamental way the presence of the spectral gap.

An exponential decay of the correlations is shown when the Ruelle operator has the spectral gap property.

A new (and natural) family of Walters potentials (on a finite alphabet derived from the Ising model) not having an exponential decay of the correlations is presented. The idea behind this result is to use the dictionary established in [9] between the termodinamic formalism and the Ising model in order to apply the Griffiths-Kelly-Sherman inequalities to obtain a superpolynomial decai correlation. Because of the lack of exponential decay, for such potentials we have the absence of the spectral gap for the Ruelle operator.

Key-words: Thermodynamic formalism, Ruelle operator, one-dimensional lattice, Analyticity of Pressure, Spectral Gap. 


\section{Sumário}

\begin{tabular}{lr}
\hline Lista de Símbolos & 12
\end{tabular}

\begin{tabular}{|lll}
1 & O MÉTODO DO OPERADOR DE TRANSFERÊNCIA & 17
\end{tabular}

1.1 O TEOREMA DE PERRON-FRÖBENIUS $\ldots \ldots \ldots \ldots \ldots \ldots \ldots$

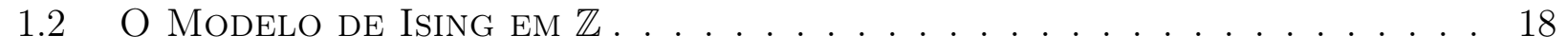

1.2 .1 O MODELO DE ISING A VOLUME FinITO . . . . . . . . . . . . . . . . 19

1.2 .2 O MODELO DE ISING UNIDIMENSIONAL (C.C. LIVRES) . . . . . . . 20

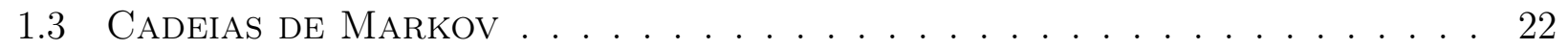

1.3 .1 CADEIAS DE MARKOV . . . . . . . . . . . . . . . . . . . 22

1.3 .2 MEDIDAS DE MARKOV . . . . . . . . . . . . . . . . . . . 23

1.4 O TEOREMA DE RUELLE-PERRON-FRÖBENIUS . . . . . . . . . . . . . . 25

1.5 APLICAÇÕES DO TEOREMA RPF . . . . . . . . . . . . . . . . . 28

1.5 .1 O FORMALISMO TERMODINÂMICO $\ldots \ldots \ldots \ldots \ldots \ldots$

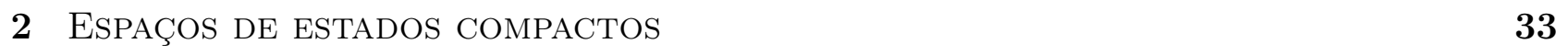

$2.1 \quad$ O ESPAÇO $M$ E MEDIDAS A PRIORI $\ldots \ldots \ldots \ldots \ldots$

$2.2 \quad$ O ESPAÇO DAS SEQUÊNCIAS $\ldots \ldots \ldots \ldots \ldots \ldots$

$2.2 .1 \quad$ OS ESPAÇOS $C(\Omega) \mathrm{E} C^{\gamma}(\Omega) \ldots \ldots \ldots \ldots \ldots \ldots$

2.3 O OPERADOR DE RUELLE $\ldots \ldots \ldots \ldots \ldots$

2.3 .1 O TEOREMA DE Ruelle-PERron-Fröbenius (RPF) . . . . . . . . 40

3 ANALITICIDADE DO FUNCIONAL PRESSÃO 49

3.1 ANALITICIDADE DO OPERADOR DE RUELLE . . . . . . . . . . . . . . . . . 49

3.2 O BuRACo EsPECTRAL E A ANALITICIDADE DA PRESSÃO . . . . . . . . . . 53

3.3 A DERIVADA DO FUNCIONAL PRESSÃO . . . . . . . . . . . . . . 56 
4 O TEOREMA DE Ruelle nO ESPAÇO DE WALters

4.1 O Operador de Ruelle nO EspaÇO DE WALTERS . . . . . . . . . . . . . . 63

4.2 O TeORema de Ruelle nO EsPAÇO DE WALTERS . . . . . . . . . . . . . . . 66

4.3 CaracterizhÇÕES DA CONDIÇÃO DE WALTERS . . . . . . . . . . . . . . . . . 70

\begin{tabular}{|llll}
5 & O OPERADOR DE RUELLE E O FORMALISMO DLR & 73
\end{tabular}

5.1 MEDIDAS DE GIBBS DUAIS . . . . . . . . . . . . . . . . . . . . . . 73

5.2 MEDIDAS DE GIBBS NO SENTIDO DLR . . . . . . . . . . . . . . . . . . . . 74

5.3 MedidAs de GibBS nO Limite TeRMOdinÂMiCO . . . . . . . . . . . . . . . . 75

$5.4 \mathcal{G}^{*} \subset \mathcal{G}^{T L} \subset \mathcal{G}^{D L R} \ldots \ldots \ldots \ldots \ldots \ldots \ldots \ldots$

\begin{tabular}{lll}
6 & DECAIMENTO DE CORRElaÇÕES & 77 \\
\hline
\end{tabular}

6.1 Buraco Espectral e Dechimento De CorrelaÇÕeS . . . . . . . . . . . . 77

6.2 Ausência de Buraco Espectral no EspaÇO DE WALters . . . . . . . . . 79

\begin{tabular}{|lr}
\hline A O FORMALISMO DLR & 87 \\
\hline
\end{tabular}

A.1 PRELIMINARES . . . . . . . . . . . . . . . . . . . . 87

A.2 ESPECIFICAÇÕES . . . . . . . . . . . . . . . . . . . . . . . . 90

A.3 $\lambda$-ESPECIFICAÇÕES . . . . . . . . . . . . . . . . . . . . . . . 91

A.4 ESPECIFICAÇÕES GIBBSSIANAS . . . . . . . . . . . . . . . . . . . 96

A.4.1 POTENCIAIS . . . . . . . . . . . . . . . . 96

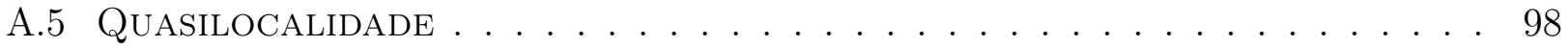

A.6 O MODELO DE ISING . . . . . . . . . . . . . . . . . . . . . . . . . . . . . . . . . . . 99

A.6.1 MOdELO DE ISING: FORMALISMO DLR . . . . . . . . . . . . . . . . . . 99

A.6.2 Modelo de Isign: Limite TERMOdinÂMiCO . . . . . . . . . . . . . . 101

A.6.3 DESIGUALDADES GKS-I E GKS-II . . . . . . . . . . . . . . . . . . . 103

\section{B Alguns Resultados Análise}

$\begin{array}{ll}\text { E TEORIA ESPECTRAL } & 109\end{array}$

B.1 ANALITICIDADE EM ESPAÇOS DE BANACH . . . . . . . . . . . . . . . . . . 109

B.2 Alguns Resultados de AnÁlise . . . . . . . . . . . . . . . . . . . . . . 110

B.3 ElEmentos DE TEORIA ESPECTRAL . . . . . . . . . . . . . . . . . . 110 


\section{LisTa DE SímBolos}

$\mathcal{G}^{*}$ : $\quad$ Medidas de Gibbs provenientes do formalismo do operador de Ruelle, página 73.

$\mathcal{G}^{D L R}$ : Medidas de Gibbs provenientes do Formalismo DLR, página 75.

$\mathcal{G}^{T L}:$ Medidas de Gibbs provenientes do Limite Termodinâmico, página 75.

$\mathcal{K}: \quad$ Subespaço normado completo de $C(\Omega)$, página 36.

$\mathcal{K}^{*}$ : Dual topológico do espaço $\mathcal{K}$, página 36 .

$\mathcal{L}(\mathcal{K}, \mathcal{K})$ : Espaço dos operadores linerares limitados de $\mathcal{K}$, página 36.

$\operatorname{Hol}(f)$ : Constante de Hölder de $f$, página 34 .

$\mathscr{L}_{f}$ : Operador de Ruelle associado ao potencial $f$, página 36.

$\mathscr{L}_{f}^{*}: \quad$ Dual do operador de Ruelle associado ao potencial $f$, página 36.

$\mu$ : $\quad$ Probabiliade definida nos Borelianos de $M$, página 33.

$\mu_{\Lambda, \beta}$ : Medida de Gibbs a volume $\Lambda$, ao inverso da temperatura $\beta>0$, página 19.

$\nu_{f}$ : $\quad$ Automedida do operador de Ruelle associado ao potencial $f$, página 41.

$\Omega: \quad$ Espaço produto $M^{\mathbb{N}}$, página 33.

$\Omega_{\Lambda}$ : Espaço de configurações a volume finito $\Lambda$, página 18.

$\pi_{T}$ : $\quad$ Projetor espectral associado ao operador $T$, página 55.

$\sigma: \quad$ Operador shift à equerda em $\Omega$, página 36 .

$\Theta$ : $\quad$ Mapa que associa o potencial $f$ ao seu operador de Ruelle $\mathscr{L}_{f}$, página 49.

$C(\Omega)$ : Espaço das funções contínuas definidas em $\Omega$, página 34 .

$C^{\gamma}(\Omega)$ : Espaço das funções Hölder contínuas em $\Omega$, página 34 .

$C_{\varphi_{1}, \varphi_{2}, \nu}(n)$ : Decaimento de correlações dos potenciais $\varphi_{1}, \varphi_{2}$, página 77.

$d_{\Omega}: \quad$ Métrica produto em $\Omega$, página 33. 
$f_{\Lambda, \beta, h}$ : Energia livre a volume $\Lambda$, ao inverso da temperatura $\beta>0$, página 20 .

$h_{f}$ : $\quad$ Autofunção do operador de Ruelle associado ao potencial $f$, página 41.

$H_{\Lambda}$ : Hamiltoniano a volume $\Lambda$, página 18.

M: $\quad$ Espaço métrico compacto, página 33.

$M_{\Lambda, \beta, h}$ : Magnetização a volume $\Lambda$, ao inverso da temperatura $\beta>0$, página 19 .

$P(f)$ : Pressão topológica do potencial $f$, página 54 .

$P_{\Lambda, \beta, h}$ : Pressão a volume $\Lambda$, ao inverso da temperatura $\beta>0$, página 20.

$W(\Omega)$ : Espaço dos potenciais satisfazendo a condição de Walters, página 63.

$Z_{\Lambda, \beta}$ : Função de partição a volume $\Lambda$, ao inverso da temperatura $\beta>0$, página 19 . 


\section{INTRODUÇÃO}

O Teorema de Ruelle-Perron-Fröbenius é um dos mais importantes resultados no formalismo termodinâmico moderno. Atualmente o operador de Ruelle tem se tornado uma ferramenta padrão em várias áreas dos sistemas dinâmicos, da Matemática e Física Matemática. A literatura a respeito do teorema de Ruelle-Perron-Fröbenius é vasta, a seguinte lista é apenas uma lista parcial de livros e artigos a respeito do assunto, [2, 6, 16, 17, 23, 37, 39, 47]. O formalismo termodinâmico clássico foi originalmente desenvolvido no espaço de Bernoulli $M^{\mathbb{N}}$, com $M$ sendo um conjunto (comumente chamado de alfabeto ou espaço de estados) finito, ver [37, 6]. Quando $M$ é finito podemos obter conjugações, via partições de Markov, de subshift no espaço simbólico com dinâmicas uniformemente hiperbólicas em variedades compactas.

O formalismo do operador de Ruelle tem sido bastante útil no contexto da análise multifractal. Bowen em um trabalho seminal em [7], estabeleceu uma relação entre a dimensão de Hausdorff de certos conjuntos fractais e a pressão topológica no contexto de dinâmicas conformes unidimensionais. Esta relação é chamada de equação de Bowen, e em trabalhos posteriores foi estendida por Manning e McCluskey para dinâmicas hiperbólicas em superfícies compactas, possibilitando o cálculo de dimensão de Hausdorff em ferraduras, para maiores detalhes veja [7, 35, 36], e também os textos introdutórios em [3, 38].

Motivações para considerar alfabetos mais gerais além dos considerados no contexto dos sistemas dinâmicos podem ser encontradas por exemplo, em [41, 43] onde são propostos modelos com um alfabeto infinito $M=\mathbb{N}$ para descrição de dinâmicas não uniformemente hiperbólicas, por exemplo, conhecidas como Manneville-Pomeau. Em Mecânica Estatística Clássica alfabetos não contáveis aparecem, por exemplo, nos modelos $O(n) \operatorname{com} n \geq 2$. Nesses modelos o alfabeto é $\mathbb{S}^{n-1}$ a esfera unitária no espaço Euclideano $\mathbb{R}^{n}$, para detalhes ver [19]. Alfabetos ilimitados, tais como os espaços Borel standard, o qual inclui alfabetos compactos e não compactos, são considerados em detalhes em [24]. Deve-se mencionar que problemas de otimização ergódica são considerados em alfabetos infinitos, veja [4, 13, 22, 41].

O trabalho de Walters em [48] marcou o inicio de três décadas de grande atividade no formalismo termodinâmico onde potenciais menos regulares que Hölder são considerados. Esta classe de potenciais é chamada de classe de Walters, alternativamente, espaço de Walters. Uma teoria bastante completa em espaços de Walters foi desenvolvida para alfabetos finitos e contáveis, ver [13. No trabalho de Walters, [48], a dinâmica é suposta misturadora e está definida em um conjunto compacto, os potenciais admitidos são potenciais com variação somável. Um dos principais objetivos desta tese de doutorado reside em estender a versão do Teorema de Ruelle obtida em [48]. Também provamos a dependência analítica do operador de Ruelle com relação ao potencial. Propriedades espectrais desse operador são estudadas e algumas consequências 
dessas propriedades, tais como existência ou não de buraco espectral, assim como a analiticidade ou não da pressão, são estabelecidas.

A dificuldade de definir o operador de Ruelle para alfabetos não contáveis é superada introduzindo-se uma medida de probabilidade a-priori em $M$. Esta estratégia é também adotada no contexto de Mecânica Estatística do equilibrio e do formalismo DLR para medida de Gibbs, ver [24].

Os principais resultados originais presentes nesta tese estão publicados no artigo Spectral Properties of the Ruelle Operator on the Walters Class over Compact Spaces, autores L. Cioletti e E. A. Silva, publicado online em 2016 em Nonlinearity, disponível em http://iopscience.iop.org/article/10.1088/0951-7715/29/8/2253.

Grande parte do Capítulo 3 é baseada também no seguinte artigo, deste autor e colaboradores The analyticity of a generalized Ruelle operator, publicado em 2014 na revista Bulletin of the Brazilian Mathematical Society, New Series, volume 45(1), páginas 53-72. Disponível em http://link.springer.com/article/10.1007/s00574-014-0040-3.

No que segue explicamos como está organizada esta tese de doutorado e quais são as contribuições originais deste trabalho.

\section{CAPÍtulo 1}

Este capítulo tem caráter essencialmente didático. Aqui, enunciaremos o Teorema de Perron Fröbenius para matrizes e abordaremos algumas de suas aplicações, tais como o cálculo da pressão no modelo de Ising, aplicações em cadeias de Markov discretas e em teoria ergódica. Depois apresentaremos um exemplo através do qual introduziremos o operador de Ruelle no contexto de alfabetos finitos. Enunciaremos o Teorema de Ruelle-Perron- Fröbenius. Falaremos brevemente de algumas de suas aplicações no formalismo termodinâmico e análise multifractal.

\section{Capítulo 2}

Neste capítulo apresentamos o operador de Ruelle agindo em $C(\Omega)$, onde $\Omega=M^{\mathbb{N}}$ e o alfabeto $M$ é um espaço métrico compacto. Uma hipótese natural sobre $M$ é que $M$ tenha base enumerável, para que assim o operador shift $\sigma: \Omega \rightarrow \Omega$ seja transitivo, propriedade fundamental na prova do Teorema de Ruelle tanto no contexto de alfabetos finitos, quanto neste novo contexto. Uma versão do Teorema de Ruelle é provada neste capítulo, quando o operador de Ruelle é definido por um potencial Hölder contínuo. Esta versão é apresentada em [1], para o caso $M=S^{1}$, o círculo unitário. Em [32], ainda para potenciais Hölder, resultados semelhantes são obtidos para o caso em que o alfabeto $M$ é espaço um métrico compacto arbitrário. A única diferença da apresentação feita neste capítulo para a apresentação feita em [1, 32] é que por motivos pessoais preferimos seguir o roteiro da demonstração feita em [37]. A diferença entre as duas abordagens difere apenas na demonstração da existência de uma autofunção positiva. O objetivo deste capítulo é familiarizar o leitor com as notações básicas, bem como com as principais técnicas utilizadas para o estudo do Operador de Ruelle.

\section{Resultados Obtidos Neste Trabalho}

Os principais resultados obtidos nesta tese de doutorado, baseada em [11], se encontram nos Capítulos 3,4,5 e 6, os quais passamos a descrever agora: 


\section{Capítulo 3}

Na Seção 3.1 deste capítulo é introduzido o conceito de subespaço invariante pelo operador de Ruelle. Mostramos que se o operador de Ruelle $\mathscr{L}_{f}$ deixa invariante uma álgebra de Banach $\mathcal{K} \subset C(\Omega)$ para todo $f \in \mathcal{K}$ então os mapas $\mathcal{K} \ni f \rightarrow \mathscr{L}_{f}$ e $\mathcal{K}^{*} \ni f \rightarrow \mathscr{L}_{f}^{*}$ são analíticos. Este resultado constitui uma versão consideravelmente mais forte dos resultados obtidos, por este autor e colaboradores em [44], onde $\mathcal{K}=C^{\gamma}(\Omega)$, e a analiticidade do dual não é discutida.

$\mathrm{Na}$ Seção 3.2 enunciamos e provamos um teorema que fornece condições suficientes para a existência de buraco espectral para o operador de Ruelle associado a potenciais Hölder contínuos. Usamos este resultado quando $\mathcal{K}=C^{\gamma}(\Omega)$ para provar a analiticidade da pressão topológica (real analítica) $P: C^{\gamma}(\Omega) \rightarrow \mathbb{R}$, o qual estende resultados análogos conhecidos em alfabetos finitos/discretos. Na Seção 3.3, a última deste capítulo a derivada do funcional pressão é calculada em todos os detalhes, ainda no contexto de espaços de estados compactos.

\section{Capítulo 4}

Neste capítulo, na Seção 4.1, introduzimos os espaços de Walters. Diferentemente do caso em que o espaço de estados é discreto, aqui, as várias maneiras de se formular a condição de Walters podem não coincidir, isso nos obriga a diferenciar entre condição de Walters e condição fraca de Walters. Como a própria nomenclatura sugere, todo potencial que satisfaz a condição de Walters satisfaz a condição fraca de Walters, entretanto a recíproca nem sempre é valida, um exemplo concreto é fornecido de modo a ilustrar esta situação. O conjunto dos potenciais satisfazendo a condição de Walters, $W(\Omega)$, chamaremos de Classe de Walters. Consideramos em $W(\Omega)$ a norma $\|\cdot\|_{W}$ introduzida em [5] a qual torna $W(\Omega)$ um espaço de Banach. Provamos que o par $\left(W(\Omega),\|\cdot\|_{W}\right)$ é uma algebra de Banach e usamos isso para provar que o espaço $W(\Omega)$ é invariante pelo operador de Ruelle. Usando estes dois fatos temos como corolário, que a aplicação $W(\Omega) \ni f \rightarrow \mathscr{L}_{f} \in L(W(\Omega), W(\Omega))$ é analítica (consequência do Capítulo 2).

Na Seção 4.2 uma versão do Teorema de Ruelle é enunciada e provada para potenciais $f$ satisfazendo a condição fraca de Walters, no contexto de espaços de estados compactos. Por uma versão queremos dizer que valem quase todas as propriedades do Teorema de Ruelle clássico para potenciais Hölder contínuos, com exceção da informação a respeito da existência do buraco espectral. Em outras palavras, não sabemos dizer se o maior autovalor obtido na prova do teorema é um polo simples. Vale salientar que mesmo no caso em que o espaço de estados é discreto, essa é a melhor versão que se conhece. Observamos que este teorema é uma generalização não trivial de alguns dos principais resultados de [1, 32] onde alfabetos compactos são considerados. A Seção 4.3, a última deste capítulo tem caráter apenas didático. Nela enunciamos de três maneiras diferentes a condição de Walters, no caso em que o alfabeto é finito e provamos que estas três condições, neste caso, são equivalentes.

\section{Capítulo 5}

Neste capítulo estudamos as relações que a medida de Gibbs proveniente do formalismo de Ruelle, i.e, as automedidas do dual do operador de Ruelle, as quais serão denotadas por $\mathcal{G}^{*}(f)$, tem com as outras noções de medidas de Gibbs, a saber, a noção de medida de Gibbs associadas ao limite termodinâmico, e as medidas de Gibbs segundo o formalismo DLR, respectivamente denotados por $\mathcal{G}^{T L}(f)$ e $\mathcal{G}^{D L R}(f)$. Mostramos que quando o potencial $f \in W(\Omega)$ então as medidas de Gibbs associadas ao operador de Ruelle também são medidas de Gibbs no sentido 
do limite termodinâmico e no sentido DLR, i.e, $\mathcal{G}^{*}(f) \subset \mathcal{G}^{T L}(f) \subset \mathcal{G}^{D L R}(f)$. Isso generaliza, em certo aspecto, um resultado de [9]. De modo mais preciso em [9] mostra-se, no contexto de alfabetos discretos, que quando o potencial $f$ satisfaz a condição de Walters todos estes conceitos de medidas de Gibbs coincidem, i.e, $\mathcal{G}^{*}(f)=\mathcal{G}^{T L}(f)=\mathcal{G}^{D L R}(f)$. A expressão "em certo aspecto" é usada acima para deixar claro que generalizamos apenas uma das direções das inclusões necessárias para a igualdade acima, a saber a direção fácil. A outra direção é aparentemente bem mais complicada e requer outros tipos de técnicas. Atualmente sabemos, através de comunicações privadas com o primeiro autor de [9], que mesmo no caso compacto a inclusão oposta também é verdadeira.

\section{Capítulo 6}

Na primeira seção deste capítulo estudamos as relações entre o buraco espectral no operador de Ruelle e a taxa de decaimento de correlações entre potenciais. Mostramos que quando o operador de Ruelle apresenta a propriedade do buraco espectral então o decaimento de correlações acontece com taxa exponecial. Na segunda seção uma "nova" família de potenciais para o qual o operador de Ruelle apresenta a ausência de buraco espectral é apresentada. A razão das aspas na palavra nova, é que esta família é nova apenas no contexto dos sistemas dinâmicos, enquanto que é uma velha conhecida na comunidade da Mecânica Estatística. A ideia para atingir o objetivo principal desta seção é exibir um par de potenciais para o qual o decaimento de correlações ocorre com taxa polinomial. Para este fim usamos vários teoremas de Mecânica Estatística, como as desigualdades de Griffiths-Kelly-Sherman (desigualdades GKS), ver [26, 27, 28, 30], entre outros resultados apresentados devidamente nos apêndices. Vale mencionar que o decaimento subexponencial obtido no Capítulo 6 não pode ser recuperado pelos resultados apresentados no trabalho em [42] a respeito de decaimentos de correlações subexponenciais nem do trabalho de Gouëzel em [25], que generaliza o anterior. Acreditamos que os exemplos apresentados no Capítulo 6 podem inspirar novas aplicações das desigualdades GKS no estudo da ausência de buraco espectral em outras situações. não pode ser recuperado pelos resultados apresentados em [42] a respeito de decaimentos de correlações subexponenciais nem do trabalho de Gouëzel em [25], que generaliza o anterior. Acreditamos que os exemplos apresentados no Capítulo 6 podem inspirar novas aplicações das desigualdades GKS no estudo da ausência de buraco espectral em outras situações. 


\section{Capítulo 1}

\section{O MÉTODO DO OpERADOR DE TRANSFERENCIA}

Este capítulo tem um caráter didático. Aqui, enunciaremos o Teorema de Perron-Fröbenius para matrizes e abordaremos algumas de suas aplicações tais como, o cálculo da pressão no modelo de Ising, Cadeias de Markov discretas e Teoria Ergódica. Depois apresentaremos um exemplo através do qual introduziremos o Operador de Ruelle. Será enunciado o Teorema de Ruelle-Perron-Fröbenius e discutiremos brevemente algumas de suas aplicações no Formalismo Termodinâmico.

\subsection{O TeOrema de Perron-FröBenius}

Antes de apresentar o enunciado do Teorema de Perron-Fröbenius vamos introduzir algumas terminologias. Uma matriz $A=\left(a_{i j}\right)$ de tamanho $n \times n$ é dita $n \tilde{a} o$ negativa se todas as suas entradas são reais e $a_{i j} \geq 0$ para todo $0 \leq i, j \leq n$. De modo análogo diremos que $A$ é positiva se todas as suas entradas são reais e $a_{i j}>0$ para todo $0 \leq i, j \leq n$. A mesma terminologia será usada para vetores em $\mathbb{C}^{n}$. Se para cada $0 \leq i, j \leq n$ existe $m(i, j)$ inteiro positivo tal que $A_{i j}^{m(i, j)}>0$ diremos $A$ é irredutivel. Se $i \in\{1, \ldots, n\}$ é tal que $A_{i i}^{n}=0$ para todo $n \geq 1$ dizemos que $i$ é um estado errante. Uma matriz $A$ será dita aperiódica quando existir um $m$ inteiro positivo tal que $A^{m}$ é uma matriz positiva. A multiplicidade geométrica de um autovalor $\lambda \in \mathbb{C}$ de uma matriz complexa $A$ de tamanho $n \times n$ é a dimensão do autoespaço $\left\{v \in \mathbb{C}^{n} ; A v=\lambda v\right\}$. A multiplicidade algébrica de $\lambda$ é a multiplicidade de $\lambda$ como raiz do polinômio característico $p(z)=\operatorname{det}(A-z I)$. Um terceiro e último conceito de multiplicidade é o índice de $\lambda$, que é a ordem de $\lambda$ como raiz do polinômio característico.

Teorema 1.1.1 (Perron-Fröbenius). Seja A uma matriz não negativa, de tamanho $n \times n$ sem estados errantes. Então A admite um autovalor $\lambda_{A}$ positivo e maximal, associado a um autovetor positivo. Se A é irredutivel então a multiplicidade algébrica de $\lambda_{A}$ é igual a 1, o autovetor correspondente é positivo, e não existe nenhum outro autovetor com todas entradas não negativas para nenhum autovalor de A. Se A é aperiódica então $\lambda_{A}$ é o único autovalor de módulo máximo. 


\section{Alguns comentários a respeito da prova do Teorema de Perron-Fröbenius}

Existem várias provas do Teorema de Perron-Fröbenius, talvez uma das mais interessantes seja a prova "dinâmica" deste teorema. A grosso modo esta prova consiste em observar como a matriz positiva $A$ age no "quadrante" positivo de $\mathbb{R}^{n}$. Inicialmente precisamos observar como as retas se comportam mediante a ação de $A$. Para isto é interessante considerar algum objeto matemático que detecte tais variações. Com este fim é introduzida a projetivização do quadrante positivo de $\mathbb{R}^{n}$ com a chamada "métrica projetiva", em relação à qual a transformação (também projetivizada) $F: \Delta \ni[x] \mapsto[(A x) /\|A\|] \in \Delta$ é uma contração. A prova é finalizada mostrando que existe um único ponto fixo $\left[x_{0}\right]$ de $F$ que satisfaz $\lim _{n \rightarrow \infty} F^{n}[y]=\left[x_{0}\right]$ para todo $[y] \in \Delta$. Veja uma apresentação detalhada desta prova em [2].

\section{MATRIZES EstocÁsTiCAS}

Uma matriz positiva $P=\left(p_{i j}\right)$ é dita uma matriz estocástica quando para todo $j=1, \ldots, n$ temos que $\sum_{i=1}^{n} P_{i j}=1$. Esta nomenclatura é motivada pela teoria dos Processos Estocásticos. Mais a frente, neste capítulo, veremos uma aplicação do Teorema de Perron-Föbenius neste contexto. O seguinte resultado a respeito de matrizes estocásticas é essencialmente um escólio da demonstração do Teorema de Perron-Fröbenius.

Corolário 1.1.2. Se P é uma matriz estocástica de tamanho $k \times k$ e aperiódica, então

(a) $\lambda=1$ é autovalor maximal de $P$;

(b) Existe um único v no simplexo unitário tal que $v P=v$;

(c) Se $w$ pertence ao simplexo unitário então $\lim _{n \rightarrow \infty} w P^{n}=v$.

\subsection{O Modelo De ISING EM $\mathbb{Z}$}

\section{O Latice Unidimensional e o EspaÇo de ConfiguraÇÕes}

Nosso modelo será descrito em intervalos simétricos $\Lambda=[-m, m] \cap \mathbb{Z}$, denotaremos a cardinalidade do conjunto $\Lambda$ por $|\Lambda|$. Cada $j \in \Lambda$ é chamado um sítio. Denotamos $\Omega_{\Lambda} \equiv\{-1,1\}^{\Lambda}$, e o conjunto $\Omega_{\Lambda}$ é dito o espaço de configurações. Um elemento genérico $\omega=\left(\omega_{i}\right)_{i \in \Lambda} \in \Omega_{\Lambda}$ é dito uma configuração. Consideraremos em $\Omega_{\Lambda}$ a sigma álgebra das partes. Também consideraremos em $\Omega_{\Lambda}$ uma topologia a qual será a topologia produto proveniente da topologia discreta em $\{-1,1\}$. Com está topologia temos que $\Omega_{\Lambda}$ é compacto. A cada sítio $j$ associamos a variável aleatória spin, $\sigma_{j}: \Omega_{\Lambda} \rightarrow\{-1,1\}$ definida por $\sigma_{j}(\omega)=\omega_{j}$.

\section{FunÇÃO DE ENERGIA}

O Hamiltoniano $H_{\Lambda}: \Omega_{\Lambda} \rightarrow \mathbb{R}$ é uma função que associa a cada configuração $\omega$ a energia correspondente $H_{\Lambda}(\omega)$. 


\subsubsection{O Modelo de Ising a Volume Finito}

Em uma de suas versões mais simples, cujas as condições de contorno são livres, o modelo de Ising é caracterizado pelo seguinte Hamiltoniano $H_{\Lambda}: \Omega_{\Lambda} \rightarrow \mathbb{R}$

$$
H_{\Lambda}(\omega)=-\sum_{\substack{\{i, j\} \subset \Lambda \\ i \neq j}} J_{i j} \omega_{i} \omega_{j}-\sum_{i \in \Lambda} \omega_{i} h_{i}
$$

onde a soma acima é feita sobre todos os pares não-ordenados de elementos de $\Lambda$ e $\left(J_{i j}\right)_{i, j \in \Lambda}$ e $\left(h_{i}\right)_{i \in \Lambda}$ são famílias de números reais fixados. As constantes $J_{i j}$ são chamadas no contexto de Mecânica Estatística de constantes de acoplamento ( ferromagnéticas quando $J_{i j} \geq 0$ ). O termo $-J_{i j} \omega_{i} \omega_{j}$ é interpretado como energia de interação entre os spins nos sítios $i$ e $j$. O número $h_{i}$ associado ao sítio $i \in \Lambda$ é interpretado como a ação de um campo magnético externo sobre este sítio.

Para completar a descrição do modelo de Ising usamos o Hamiltoniano acima, para definir uma medida de probabilidade que fornecerá a probabilidade de uma configuração $\omega \in \Omega_{\Lambda}$ ocorrer. Para isso seguiremos a tradição da termodinâmica. Para pesar as influências da aleatoriedade e da minimização de energia, a probabilidade de ocorrência de uma certa configuração $\omega$ deve diminuir exponencialmente com sua energia $H_{\Lambda}(\omega)$. Por razões que não serão discutidas neste texto estas observações nos leva a seguinte medida de probabilidade

$$
\mu_{\Lambda, \beta}(\{\omega\})=\frac{\exp \left[-\beta H_{\Lambda}(\omega)\right]}{\sum_{\omega \in \Omega_{\Lambda}} \exp \left[-\beta H_{\Lambda}(\omega)\right]}
$$

onde a constante $Z_{\Lambda, \beta}=\sum_{\omega \in \Omega_{\Lambda}} \exp \left[-\beta H_{\Lambda}(\omega)\right]$ é uma constante de normalização chamada função de partição, e a constante $\beta=1 / T$ é o inverso da temperatura. A medida de probabilidade dada por 1.2 é chamada a medida de Gibbs no volume $\Lambda$ ao inverso da temperatura $\beta>0$.

As razões que levam a definição 1.2 de medida de Gibbs provém da Física, e não entraremos no mérito de apresentá-las. Algumas explicações são esotéricas, outras adotam um ponto de vista matemático completamente axiomático. Para o leitor interessado na abordagem matemática, sugerimos a leitura do Capítulo 1, Seção A de [6] e a referência [29].

\section{Pressão, Energia Livre e MagnetizaÇÃo}

Nesta subseção assumiremos que o campo magnético $\left(h_{i}\right)_{i \in \Lambda}$ é constante, isto é, $h_{i} \equiv h$ para todo $i \in \Lambda$, para todo $\Lambda \subset \mathbb{Z}$. Seja $S_{\Lambda}$ a variável aleatória que em cada configuração $\omega \in \Omega_{\Lambda}$ é dada pela soma finita $S_{\Lambda}(\omega)=\sum_{i \in \Lambda} \omega_{i}$. A magnetização é definida como sendo o valor esperado de $S_{\Lambda}$ com relação à medida de Gibss (1.2),

$$
M_{\Lambda, \beta, h}=\int_{\Omega_{\Lambda}} S_{\Lambda}(\omega) d \mu_{\Lambda, \beta}(\omega)=\sum_{i \in \Lambda} \sum_{\omega \in \Omega_{\Lambda}} \omega_{i} \cdot \frac{\exp \left[-\beta H_{\Lambda}(\omega)\right]}{\sum_{\omega \in \Omega_{\Lambda}} \exp \left[-\beta H_{\Lambda}(\omega)\right]} .
$$

As grandezas

$$
P_{\Lambda, \beta, h} \equiv \frac{\ln \left(Z_{\Lambda, \beta}\right)}{|\Lambda|} \quad \text { e } \quad f_{\Lambda, \beta, h}=-\frac{1}{\beta} \frac{\ln \left(Z_{\Lambda, \beta}\right)}{|\Lambda|}
$$


são chamadas pressão e energia livre respectivamente, a volume finito. A pressão e a energia live são potenciais muito interessantes, pois deles podemos derivar grandezas macroscópicas relevantes. Por exemplo a magnetização $M_{\Lambda, \beta, h}$ pode ser obtida por $M_{\Lambda, \beta, h}=-\frac{\partial f_{\Lambda, \beta, h}}{\partial h}$. Ou seja, a pressão e a energia livre podem trazer consigo informações a respeito do fenômeno de transição de fase.

\subsubsection{O MODElO DE ISING UNidimensional (CONDIÇÃO DE CONTORNO LIVRES)}

Nesta seção, mostraremos como usar o Teorema de Perron-Fröbenius para fazer o cálculo explícito da pressão de um modelo de Ising com algumas simplificações. A saber, consideraremos $\Lambda_{N}$ como sendo o intervalo simétrico de números inteiros $[-N, N] \cap \mathbb{Z}, J_{i j}=J$ para todo $i, j \in \Lambda_{N}$ tal que $|i-j|=1$ e $J_{i j}=0$, caso contrário. Vamos tomar $h_{i}=h$ para todo $i \in \Lambda$. Assim o Hamiltoniano que associa a cada configuração $\omega \in \Omega_{\Lambda_{N}}$ sua energia será dado por

$$
H_{\Lambda_{N}}(\omega)=-\sum_{i=-N}^{N-1} J \omega_{i} \omega_{i+1}-\sum_{i=-N}^{N} h \omega_{i} .
$$

Nosso primeiro passo no intuito de calcular a pressão do modelo de Ising é reescrever o Hamiltoniano $H_{\Lambda_{N}}$ de modo conveniente,

$$
\begin{aligned}
H_{\Lambda_{N}}(\omega) & =-\sum_{i=-N}^{N-1} J \omega_{i} \omega_{i+1}-h \sum_{i=-N}^{N} \omega_{i} \\
& =-\sum_{i=-N}^{N-1} J \omega_{i} \omega_{i+1}-\frac{h}{2} \sum_{i=-N}^{N-1}\left(\omega_{i}+\omega_{i+1}\right)-\frac{h}{2}\left(\omega_{-N}+\omega_{N}\right) .
\end{aligned}
$$

Defina $\nu=\beta J$ e $B=\beta h$, então temos a seguinte expressão para a função de partição,

$$
\begin{aligned}
Z_{\Lambda_{N}}(\beta, J, h) & =\sum_{\omega \in \Omega_{\Lambda_{N}}} \exp \left[-\beta H_{\Lambda_{N}}(\omega)\right] \\
& =\sum_{\omega \in \Omega_{\Lambda_{N}}} \prod_{i=-N}^{N-1} \exp \left[\nu \omega_{i} \omega_{i+1}+B / 2\left(\omega_{i}+\omega_{i+1}\right)\right] \exp \left[B / 2\left(\omega_{-N}+\omega_{N}\right)\right] .
\end{aligned}
$$

Definindo,

$$
L=L\left(\omega_{i}, \omega_{j}\right)=\left(\begin{array}{cc}
L(+,+) & L(+,-) \\
L(-,-) & L(-,+)
\end{array}\right)=\left(\begin{array}{cc}
e^{\nu+B} & e^{-\nu} \\
e^{-\nu} & e^{\nu-B}
\end{array}\right)
$$

temos que

$$
Z_{\Lambda_{N}}(\beta, J, h)=\sum_{\omega \in \Omega_{\Lambda_{N}}} \prod_{i=-N}^{N-1} L\left(\omega_{i}, \omega_{j}\right) \exp \left[\frac{B}{2}\left(\omega_{-N}+\omega_{N}\right)\right] .
$$

Após fazermos alguma álgebra é possível eliminar a dependência de $Z_{\Lambda_{N}}(\beta, J, h)$ nos sítios $-N+1 \leq i \leq N-1$, obtendo

$$
Z_{\Lambda_{N}}(\beta, J, h)=\sum_{\omega_{-N}= \pm, \omega_{N}= \pm} L^{2 N}\left(\omega_{-N}, \omega_{N}\right) \exp \left[\frac{B}{2}\left(\omega_{-N}+\omega_{N}\right)\right] .
$$


Podemos escrever convenientemente $Z_{\Lambda_{N}}(\beta, J, h)$ como o produto interno

$$
Z_{\Lambda_{N}}(\beta, J, h)=\left\langle\Psi, L^{2 N} \Psi\right\rangle
$$

onde $\Psi=\left(e^{B}, e^{-B}\right)$. Segue do teorema de Perron Fröbenius que $L$ tem dois autovalores reais $0<\lambda_{-}<\lambda_{+}$. Sejam $\Pi_{-}$e $\Pi_{+}$as projeções associadas aos autoespaços associados a $\lambda_{-}$e $\lambda_{+}$ respectivamente. Então podemos escrever

$$
L^{2 N}=\lambda_{-}^{2 N} \Pi_{-}+\lambda_{+}^{2 N} \Pi_{+}
$$

Deste modo a função de partição $Z_{\Lambda_{N}}(\beta, J, h)$ toma a seguinte forma:

$$
\begin{aligned}
Z_{\Lambda_{N}}(\beta, J, h) & =\left\langle\Psi, L^{2 N} \Psi\right\rangle=\lambda_{-}^{2 N}\left\langle\Psi, \Pi_{-} \Psi\right\rangle+\lambda_{+}^{2 N}\left\langle\Psi, \Pi_{+} \Psi\right\rangle \\
& =\lambda_{+}^{2 N}\left[\left\langle\Psi, \Pi_{+} \Psi\right\rangle+\frac{\lambda_{-}^{2 N}}{\lambda_{+}^{2 N}}\left\langle\Psi, \Pi_{-} \Psi\right\rangle\right]
\end{aligned}
$$

tomando o logaritmo na expressão acima, em seguida dividindo por $\left|\Lambda_{N}\right|=2 N+1$ e tomando o limite, quando $N \rightarrow \infty$ (conhecido como Limite Termodinâmico) e lembrando que $0<\lambda_{-}<\lambda_{+}$ temos que existe o seguinte limite (chamado de pressão a "volume infinito" do modelo de Ising)

$$
\begin{aligned}
p(\beta, J, h) & \equiv \lim _{N \rightarrow \infty} P_{\Lambda, \beta, h} \\
& =\lim _{N \rightarrow \infty}\left[\frac{2 N}{2 N+1} \ln \left(\lambda_{+}\right)+\frac{1}{2 N+1} \ln \left(\left\langle\Psi, \Pi_{+} \Psi\right\rangle+\frac{\lambda_{-}^{2 N}}{\lambda_{+}^{2 N}}\left\langle\Psi, \Pi_{-} \Psi\right\rangle\right)\right] \\
& =\ln \left(\lambda_{+}\right) .
\end{aligned}
$$

\section{Campo Magnético Nulo}

Supondo no exposto acima que o campo magnético $h \equiv 0$ é sempre nulo, neste caso basta fazer $B=0$ na matriz de transferência, obtemos que a matriz de transferência se reduz a

$$
L_{0}=\left(\begin{array}{cc}
e^{\beta J} & e^{-\beta J} \\
e^{-\beta J} & e^{\beta J}
\end{array}\right)
$$

Os autovetores de $L_{0}$ são $v_{+}=(1 / \sqrt{2}) \cdot(1,1)$ e $v_{-}=(1 / \sqrt{2}) \cdot(1,-1)$, com os respectivos autovalores $\lambda_{+}=e^{\beta J}+e^{-\beta J}$ e $\lambda_{-}=e^{\beta J}-e^{-\beta J}$. Como provamos que a pressão é o log do maior autovalor de $L_{0}$ temos que

$$
p(\beta, J, h=0)=\ln \left(\lambda_{+}\right)=\ln [2 \cosh (\beta J)] .
$$

Para o leitor interessado, a exposição acima foi baseada em [20]. 


\subsection{AplicaÇÕES EM TeOria ERGÓdica E CADEIAS DE MARKOV}

\subsubsection{CADEIAS DE MARKOV}

Seja $\mathcal{S}$ um conjunto enumerável, diremos que um processo estocástico $\left\{X_{n}\right\}_{n \in \mathbb{N}}$ em $(\Omega, \mathcal{F}, P)$, tomando valores em um conjunto $\mathcal{S}$ discreto é chamado de uma cadeia de Markov com espaço de estados discreto, quando para todo $i_{1}, i_{2}, \ldots, i_{n+1} \in \mathcal{S}$ temos que

$$
P\left(X_{n+1}=i_{n+1} \mid X_{0}=i_{0}, \ldots, X_{n}=i_{n}\right)=P\left(X_{n+1}=i_{n+1} \mid X_{n}=i_{n}\right) .
$$

Nos referimos a expressão 1.3 como a propriedade de Markov. Denotaremos a quantidade do lado direito da equação acima por $P_{i_{n}, i_{n+1}}^{n}$, isto é,

$$
P_{i_{n}, i_{n+1}}^{n}=P\left(X_{n+1}=i_{n+1} \mid X_{n}=i_{n}\right) .
$$

As probabilidades condicionais $P_{i_{n}, i_{n+1}}^{n}$ são chamadas probabilidades de transição a 1 passo no tempo $n$. A matriz $P(n)$ cujo termo geral $P_{i j}^{n}$ é dado por $P_{i j}^{n}=P\left(X_{n+1}=j \mid X_{n}=i\right)$ é chamada de matriz de transição a 1 passo no tempo $n$. Uma cadeia de Markov $\left\{X_{n}\right\}_{n \in \mathbb{N}}$ é chamada de cadeia de Markov homogênea, ou cadeia de Markov com probabilidades de transição estacionárias se as probabilidades de transição a 1 passo são independentes de $n$, isto é, para todo $k=1,2 \ldots$ vale

$$
P\left(X_{n+1}=i_{n+1} \mid X_{n}=i_{n}\right)=P\left(X_{n+k+1}=i_{n+1} \mid X_{n}=i_{n+k}\right) .
$$

Restringiremos nossa atenção à cadeias de Markov homogêneas. Passaremos a usar as seguintes notações $P_{i j} \equiv P_{i j}^{n}$ e $P \equiv P(n)$. No que segue será útil também considerar a distribuição inicial $\left\{p_{0}(i), i \in \mathcal{S}\right\}$ de $X$, i.e.,

$$
p_{0}(i)=P\left[X_{0}=i\right], \quad i \in \mathcal{S} .
$$

Claramente $\left\{p_{0}(i), i \in \mathcal{S}\right\}$ é um vetor de probabilidades, i.e, $\sum_{i} p_{0}(i)=1$.

\section{Matrizes DE TRANSIÇÃO A $n$-PASSOS}

Seja $\left\{X_{n}\right\}_{n \in \mathbb{N}}$ uma cadeia de Markov homogênea com espaço de estados $\mathcal{S}$. Definimos as probabilidades de transição a n passos da cadeia de Markov $\left\{X_{n}\right\}_{n \in \mathbb{N}}$ como sendo as quantidades $P_{i j}^{(n)}$ definidas por

$$
P_{i j}^{(n)}=P\left[X_{m+n}=j \mid X_{m}=i\right]=P\left[X_{n}=j \mid X_{0}=i\right] \quad \forall m \in \mathbb{N}
$$

denotamos por $P^{(n)}$ a matriz de transição de n-passos associada à cadeia de Markov $\left\{X_{n}\right\}_{n \in \mathbb{N}}$.

Proposição 1.3.1 (Equações de Chapman-Kolmogorov). Seja $\left\{X_{n}\right\}_{n \in \mathbb{N}}$ uma cadeia de Markov homogênea com distribuição inicial $\left\{p_{0}(i), i \in \mathcal{S}\right\}$ e probabilidades de transição a $n$ passos $P^{(n)}=\left(P_{i j}^{(n)}\right)$. Então para todo $n, m \in \mathbb{N}$ temos

$$
P^{(m+n)}=P^{(n)} P^{(m)}=P^{m+n}
$$

onde $P=\left(P_{i j}\right)$ é a matriz de transição a um passo da cadeia $\left\{X_{n}\right\}_{n \in \mathbb{N}}$. 


\section{ERGODICIDADE E PROBABILIDADES EstaCIONÁRIAS}

Definição 1.3.2 (Ergodicidade). Seja $P=\left(P_{i j}\right)_{i, j \in \mathcal{S}}$ a matriz de transição de uma cadeia de Markov $\left\{X_{n}\right\}_{n \in \mathbb{N}}$ com espaço de estados $\mathcal{S}$ discreto. Se para todo $j \in \mathcal{S}$ o limite

$$
\lim _{n \rightarrow \infty} P_{i j}^{n}=\pi_{j}
$$

existe, é independente de $i$ e $\sum_{j \in \mathcal{S}} \pi_{j}=1$, dizemos que a cadeia de Markov $\left\{X_{n}\right\}_{n \in \mathbb{N}}$ é ergódica.

Observe que se a cadeia de Markov $\left\{X_{n}\right\}_{n \in \mathbb{N}}$ é ergódica então segue das propriedades elementares da esperança condicional e do Teorema da Convergência Dominada que

$$
\lim _{n \rightarrow \infty} P\left[X_{n}=j\right]=\lim _{n \rightarrow \infty} \sum_{i \in \mathcal{S}} p_{0}(i) P_{i j}^{n}=\sum_{i \in \mathcal{S}} p_{0}(i) \pi_{j}=\pi_{j}
$$

Portanto no caso ergódico existe uma distribuição conhecida como distribuição de equilíbrio, dada pelo vetor $\left\{\pi_{j}, j \in \mathcal{S}\right\}$. A distribuição de equilíbrio é também conhecida como distribuição limite.

Definição 1.3.3 (Distribuição estacionária). Uma distribuição $\Pi=\left\{\pi_{j}, j \in \mathcal{S}\right\}$ é chamada uma distribuição estacionária para o processo de Markov $\left\{X_{n}\right\}_{n \in \mathbb{N}}$, com matriz de transição $P=\left(P_{i j}\right)_{i, j \in \mathcal{S}}$, se temos $\Pi P=\Pi$, ou mais explicitamente

$$
\pi_{j}=\sum_{i \in \mathcal{S}} \pi_{i} P_{i j}
$$

para todo $j \in \mathcal{S}$.

Teorema 1.3.4. Uma cadeia de Markov $\left\{X_{n}\right\}_{n \in \mathbb{N}}$ com espaço de estados $\mathcal{S}$ finito, homogênea, irredutivel e aperiódica tem uma única distribuição estacionária $\Pi=\left(\pi_{i}\right)_{i \in \mathcal{S}}$ e além do mais a cadeia de Markov $\left\{X_{n}\right\}_{n \in \mathbb{N}}$ é ergódica.

Demonstração. Seja $k \equiv|\mathcal{S}|$. Se $\underline{q}=(0, \ldots, \underbrace{1}_{i \text {-ésima }}, \ldots, 0)$ então $\underline{q} P^{n}$ é igual a $i$-ésima linha de $P^{n}$, ou seja, $q P^{n}=\left(P_{i 1}^{n}, \ldots, P_{i k}^{n}\right)$. Pelo item $(c)$ do Corolário 1.1 .2 do Teorema de PerronFröbenius temos que

$$
\left(P_{i 1}^{n}, \ldots, P_{i k}^{n}\right) \rightarrow\left(\pi_{1}, \ldots, \pi_{k}\right)
$$

quando $n \rightarrow \infty$. Em particular, $P^{n}$ converge para uma matriz cujas linhas são todas iguais ao vetor $\Pi$.

\subsubsection{MEDIDAS DE MARKOV}

Antes de prosseguir, relembrarmos o importante conceito de medida invariante. Sejam $(X, \mathcal{A}, \mu)$ um espaço de medida e $T: X \rightarrow X$ uma transformação mensurável. Dizemos que a medida $\mu$ é invariante por $T$ se para todo $A \in \mathcal{A}$ temos $\mu\left(T^{-1} A\right)=\mu(A)$. 
Uma classe de medidas invariantes para o shift à esquerda (agindo em $M^{\mathbb{N}}$ ) que desempenha um papel muito importante é a classe das Medidas de Markov que passaremos a descrever agora. Sejam $r \in \mathbb{N}$ fixado e $M \equiv\{0, \ldots, r-1\}$. Dada uma matriz estocástica $P$ de tamanho $r \times r$, positiva e aperiódica sabemos do Corolário 1.1.2 que existe um único autovetor de probabilidade $\Pi$ tal que $\Pi P=\Pi$. A medida de Markov $\mu_{P}$, será definida da seguinte maneira. Se $\left[a_{0}, \ldots, a_{k}\right]$ denota um cilindro em $M^{\mathbb{N}}$ definimos

$$
\mu_{P}\left(\left[a_{0}, \ldots, a_{k}\right]\right)=\pi_{a_{0}} P_{a_{0} a_{1}} \cdots P_{a_{k-1} a_{k}} .
$$

Note que $\mu_{P}$ se estende a álgebra gerada pelos cilindros de maneira natural, isto é, definindo a medida de uma união disjunta de cilindros como sendo a soma das medidas dos cilindros que compõe esta união. Observe também que a seguinte condição de compatibilidade é satisfeita

$$
\begin{aligned}
\sum_{j=0}^{r-1} \mu_{P}\left(\left[j, a_{0}, \ldots, a_{k}\right]\right) & =\sum_{j=0}^{r-1} \pi_{j} P_{j a_{0}} P_{a_{0} a_{1}} \cdots P_{a_{k-1} a_{k}} \\
& =\underbrace{\left(\sum_{j=0}^{r-1} \pi_{j} P_{j a_{0}}\right)}_{\text {igual a } \pi_{a_{0}}, \text { pois } \Pi \text { é autovetor }} P_{a_{0} a_{1}} \cdots P_{a_{k-1} a_{k}} \\
& =\pi_{a_{0}} P_{a_{0} a_{1}} \cdots P_{a_{k-1} a_{k}} \\
& =\mu_{P}\left(\left[a_{0}, \ldots, a_{k}\right]\right) .
\end{aligned}
$$

Portanto podemos aplicar o Teorema da Extensão de Kolmogorov para estender $\mu_{P}$ a uma medida de probabilidade nos Borelianos de $M^{\mathbb{N}}$. A medidas de probabilidade obtidas desse modo são chamadas de medidas de Markov.

Sejam $P=\left(P_{i j}\right)$ uma matriz estocástica de tamanho $r \times r$, positiva e aperiódica. Como acima vamos denotar por $\Pi=\left(\pi_{1}, \ldots, \pi_{r}\right)$ o único autovetor de probabilidade da matriz $P$ satisfazendo $\Pi P=\Pi$. Seja $\mu_{P}$ a medida de markov associada a $P$, definida no parágrafo anterior. Sejam $A=\left[a_{1}, \ldots, a_{l}\right]$ e $B=\left[b_{1}, \ldots, b_{k}\right]$ cilindros em $M^{\mathbb{N}}$. Então para todo $n \in \mathbb{N}$ temos

$$
\sigma^{-n} A=\bigcup_{\left(x_{i_{1}}, \ldots, x_{i_{n}}\right) \in\{0, \ldots, r-1\}^{n}}\left[x_{i_{1}}, x_{i_{2}}, \ldots, x_{i_{n}} a_{1}, \ldots a_{l}\right]
$$

e para $n>k$ temos

$$
\sigma^{-n} A \cap B=\bigcup_{\left(x_{k+1}, \ldots, x_{n}\right) \in\{0, \ldots, r-1\}^{n-k}}\left[b_{1}, \ldots, b_{k}, x_{k+1}, \ldots, x_{n}, a_{1}, \ldots a_{l}\right]
$$

Portanto

$$
\begin{aligned}
\mu_{P}\left(\sigma^{-n} A \cap B\right) & =\underbrace{\sum_{x_{k+1}, \ldots, x_{n}} \pi_{b_{1}} P_{b_{1} b_{2}} \cdots P_{b_{k-1} b_{k}} P_{b_{k} x_{k+1}} \cdots P_{x_{n} a_{1}} P_{a_{1} a_{2}} \cdots P_{a_{l-1} a_{l}}}_{\mu_{P}(B)} \\
& =\underbrace{\pi_{b_{1}} P_{b_{1} b_{2}} \cdots P_{b_{k-1} b_{k}}}_{P_{b_{k} a_{1}}^{n-k-1}} \cdot \underbrace{\sum_{x^{\prime}}}_{\sum_{x_{k+1}, \ldots, x_{n}} P_{b_{k} x_{k+1}} \cdots P_{x_{n} a_{1}} P_{a_{1} a_{2}} \cdots P_{a_{l-1} a_{l}} .}
\end{aligned}
$$


Pelo teorema de Perron Fröbenius temos que $P_{b_{k} a_{1}}^{n-k-1} \rightarrow \pi_{a_{1}}$ quando $n \rightarrow \infty$. Tomando o limite, quando $n \rightarrow \infty$, na expressão que aparece após a primeira chave acima temos que

$$
\sum_{x_{k+1}, \ldots, x_{n}} P_{b_{k} x_{k+1}} \cdots P_{x_{n} x_{a_{1}}} P_{a_{1} a_{2}} \cdots P_{a_{l-1} a_{l}} \rightarrow \mu_{P}(A)
$$

donde concluímos que $\lim _{n \rightarrow \infty} \mu_{P}\left(\sigma^{-n} A \cap B\right)=\mu_{P}(A) \mu_{P}(B)$.

\subsection{O operador de Ruelle e o TeOrema de RUELLE-PERRON-FrÖBENIUS}

\section{PEnSANDO DE UM MOdo UM POUCO MAIS GERAL}

Como devemos proceder se quisermos trabalhar com Hamiltonianos mais gerais que aqueles que aparecem na Seção 1.2? Na verdade existem várias alternativas. Vamos fazer a seguinte escolha. Vamos substituir o espaço de estados $\{-1,1\}$ pelo espaço $M=\{0, \ldots, n-1\}$, onde $n \in \mathbb{Z}$ é um número maior ou igual que 2 fixado. Antes de definir a energia do sistema introduzimos a seguinte terminologia: uma configuração $\omega \in M^{\mathbb{Z}}$ está no estado $k \in\{0, \ldots, n-1\}$ no sítio $i \in \mathbb{Z}$ se $\omega_{i}=k$. Agora podemos discutir como vamos definir a energia do sistema. Vamos considerar que

(1) cada sítio $i \in \mathbb{Z}$ no estado $k \in\{0, \ldots, n-1\}$ contribui com $\Phi_{0}(k)$ à energia total do sistema;

(2) se $k_{1}$ e $k_{2}$ são os estados nos sítio $i_{1}$ e $i_{2}$, respectivamente, a energia de interação $\Phi^{\star}\left(i_{1}, i_{2} ; k_{1}, k_{2}\right)$ entre eles deve depender somente da posição relativa dos sítios $i_{1}, i_{2}$ e dos estados $k_{1}$ e $k_{2}$. Isto é, existe uma função $\Phi: \mathbb{Z} \times\{0, \ldots, n-1\} \times\{0, \ldots, n-1\} \rightarrow \mathbb{R}$ tal que

$$
\Phi^{\star}\left(i_{1}, i_{2} ; k_{1}, k_{2}\right)=\Phi\left(i_{1}-i_{2}, k_{1}, k_{2}\right) ;
$$

(3) Toda a energia do sistema é devido à contribuições da forma (1) e (2).

Segundo as considerações acima a energia necessária para observar o estado $\omega_{0}$ no sitio 0 é formalmente dada pela seguinte expressão:

$$
f(\omega)=\Phi_{0}\left(\omega_{0}\right)+\sum_{j \in \mathbb{Z} \backslash\{0\}} \frac{1}{2} \Phi\left(j ; \omega_{0}, \omega_{j}\right) .
$$

Defina $\left\|\Phi^{\star}\right\|_{j}=\sup _{k_{1}, k_{2}}\left|\Phi\left(j, k_{1}, k_{2}\right)\right|$ e assuma que $\sum_{j=-\infty}^{\infty}\left\|\Phi^{\star}\right\|_{j}<\infty$. Sob estas hipóteses podemos mostrar que $f(\omega) \in \mathbb{R}$ e que $f$ é contínua em $M^{\mathbb{Z}}$.

Vamos agora fixar nossa atenção nos sítios $-m, \ldots, 0, \ldots, m$. Considerando apenas estes sítios temos um sistema finito com $n^{2 m+1}$ configurações possíveis. Levando em conta as considerações acima a energia de uma configuração $\omega_{-m} \cdots \omega_{0} \cdots \omega_{m}$ arbitrária é dada por

$$
H_{m}\left(\omega_{-m}, \cdots, \omega_{m}\right)=\sum_{j=-m}^{m} \Phi_{0}\left(\omega_{j}\right)+\sum_{-m \leq j<k \leq m} \Phi\left(k-j ; \omega_{k}, \omega_{j}\right) .
$$


Associada a $H_{m}\left(\omega_{-m}, \cdots, \omega_{m}\right)$ temos a distribuição de Gibbs $\mu_{m}$ que associa à configuração $\left(\omega_{-m}, \cdots, \omega_{m}\right)$ uma probabilidade proporcional a $e^{-\beta H_{m}\left(\omega_{-m}, \cdots, \omega_{m}\right)}$. Suponha que para cada configuração $\omega_{-m}, \cdots, \omega_{m}$ o limite

$$
\mu\left(\omega_{-m}, \cdots, \omega_{m}\right)=\lim _{k \rightarrow \infty}\left\{\mu_{k}\left(\omega_{-k}^{\prime}, \cdots, \omega_{k}^{\prime}\right): \quad \omega_{k}^{\prime}=\omega_{i}, \quad|j| \leq m\right\}
$$

existe. Então $\mu \in \mathcal{M}\left(M^{\mathbb{Z}}\right)$ e é natural chamar $\mu$ de uma distribuição de Gibbs em $M^{\mathbb{Z}}$. Se $\omega=\left(\omega_{i}\right)_{i \in \mathbb{Z}}$ é dado, uma descrição mais fisicamente razoável do sistema seria obtida se ao invés de $H_{m}\left(\omega_{-m}, \cdots, \omega_{m}\right)$, considerássemos também todas as outras contribuições das iterações dos $\omega_{j},-m \leq j \leq m$ com os outros $\omega_{k}^{\prime} s$, isto é,

$$
\tilde{H}_{m}\left(\omega_{-m}, \cdots, \omega_{m}\right)=\sum_{j=-m}^{m}\left(\Phi_{0}\left(\omega_{j}\right)+\sum_{k=-\infty}^{\infty} \frac{1}{2} \Phi\left(k-j ; \omega_{k}, \omega_{j}\right)\right) .
$$

Usando o shift podemos reescrever (1.6) como $\sum_{j=-m}^{m} f\left(\sigma^{i} \omega\right)$. Note que a diferença entre as expressões 1.6 e 1.5 é cotada superiormente por

$$
\sum_{j=-m}^{m}\left(\sum_{k=m-j+1}^{\infty} \frac{1}{2}\left\|\Phi^{\star}\right\|_{k}+\sum_{k=-\infty}^{-m+j-1} \frac{1}{2}\left\|\Phi^{\star}\right\|_{k}\right) \leq(2 m+1) \sum_{k=-\infty}^{\infty}\left\|\Phi^{\star}\right\|_{k} \equiv C
$$

então (1.6) e (1.5) diferem por no máximo $C$.

O que acontece se usarmos $S_{m} \varphi(\omega)=\sum_{j=-m}^{m} f\left(\sigma^{i} \omega\right)$ no lugar de $H_{m}$ no cálculo da distribuição de Gibbs? As probabilidades devem diferir por fatores que estão no intervalo $\left[e^{-C}, e^{C}\right]$. Seja $Z_{m}$ a função de partição associada ao Hamiltoniano original $H_{m}$ e $\tilde{Z}_{m}$ a função de partição associada ao Hamiltoniano alterado $\tilde{H}_{m}=\sum_{j=-m}^{m} f\left(\sigma^{i}(\cdot)\right)$, fixe uma configuração $\omega$ fora das primeiras coordenadas $-m, \ldots, 0, \ldots, m$, então

$$
\tilde{Z}_{m}(\omega)=\sum_{\omega^{\prime} ; \omega_{i}^{\prime}=\omega_{i},|i|>m} e^{-\beta \tilde{H}\left(\omega^{\prime}\right)}=\sum_{\omega^{\prime} ; \omega_{i}^{\prime}=\omega_{i}, \quad|i|>m} e^{-\beta S_{n} f\left(\omega^{\prime}\right)}
$$

levando em conta a discussão do parágrafo anterior temos a seguinte desigualdade envolvendo as funções de partições acima,

$$
e^{-\beta C} Z_{m}(\omega) \leq \tilde{Z}_{m}(\omega) \leq e^{\beta C} Z_{\Lambda}(\omega)
$$

Tomando o ln em ambos os membros e dividindo por $2 m+1$ concluímos que quando existem os limites abaixo que eles coincidem

$$
\lim _{|\Lambda| \rightarrow \infty} \frac{\ln \left(\tilde{Z}_{\Lambda}\right)}{|\Lambda|}=\lim _{|\Lambda| \rightarrow \infty} \frac{\ln \left(Z_{\Lambda}\right)}{|\Lambda|}=\lim _{|\Lambda| \rightarrow \infty} P_{\Lambda, \beta, h} .
$$

\section{O OPERADOR DE RUELLE}

A partir daqui seguiremos a tradição dos sistemas dinâmicos, e passaremos a usar as letras do alfabeto latino para designar uma configuração do sistema. Então ao invés de usar $\omega, \omega^{\prime}$ ou $\tilde{\omega}$ 
passaremos a utilizar as letras $\underline{x}, \underline{y}, \underline{z}$, etc. Toda a discussão acima nos motiva a considerar um novo objeto de estudo, a saber, o operador de Ruelle.

Seja $C(\Omega)$ o espaço das funções contínuas $\Omega$ em $\mathbb{R}$. Definimos para cada $f \in C(\Omega)$ o operador de Ruelle $\mathscr{L}_{f}: C(\Omega) \rightarrow C(\Omega)$ pondo

$$
\mathscr{L}_{f} \varphi(\underline{x})=\sum_{\sigma(\underline{y})=\underline{x}} \varphi(\underline{y}) e^{f(\underline{y})} .
$$

Refazendo toda a discussão da subsecção anterior, usando o látice $\mathbb{N}$ ao invés de $\mathbb{Z}$ chegaremos à seguinte conclusão, se $\tilde{f}=\beta f$ e 1 é a função constante igual a 1 então podemos escrever a função de partição alterada como

$$
\tilde{Z}_{m}(\underline{x})=\mathscr{L}_{\tilde{f}}^{m}(\mathbf{1})(\underline{x})
$$

Esta observação é de extrema importância, pois mostra que sob hipóteses bastante razoáveis podemos sair do mundo mensurável para o mundo topológico, e ter à nossa disposição ferramentas poderosas de análise. Temos a seguinte versão do Teorema de Perron-Föbenius em dimensão infinita:

Teorema 1.4.1 (Teorema de Ruelle-Perron-Fröbenius). Seja $f \in C^{\gamma}(\Omega)$ e considere o operador de Ruelle associado $\mathscr{L}_{f}: C(\Omega) \rightarrow C(\Omega)$. Então

(a) existe um número real $\lambda_{f}>0$, uma função positiva $h_{f}$ e uma medida $\nu_{f} \in \mathcal{M}(\Omega)$ para os quais $\mathscr{L}_{f} h_{f}=\lambda_{f} h_{f}, \quad \int h_{f} d \nu_{f}=1 e$

$$
\lim _{m \rightarrow \infty}\left\|\lambda^{-m} \mathscr{L}_{f}^{m} \varphi-h_{f} \int \varphi d \nu_{f}\right\|_{0}=0
$$

(b) o espectro de $\mathscr{L}_{f}: C^{\gamma}(\Omega) \rightarrow C^{\gamma}(\Omega)$ consiste de $\lambda_{f}$ e de uma porção contida em um disco de raio estritamente menor que $\lambda_{f}$.

A medida $m_{f}=h_{f} \nu_{f}$ é uma medida invariante para o shift e é dita uma medida de Gibbs. Temos os seguintes corolários

Corolário 1.4.2. As seguintes afirmações a respeito da medida de Gibbs $m_{f}$ são verdadeiras,

(a) $m_{f}$ é uma medida invariante para o shift $\sigma: \Omega \rightarrow \Omega$;

(b) O par $\left(\sigma, m_{f}\right)$ é misturador (e portanto $m_{f}$ é ergódica);

(c) A medida $m_{f}$ tem suporte total.

Corolário 1.4.3. Seja $f \in C^{\gamma}(\Omega)$, temos para todo $\underline{x} \in \Omega$ que

$$
\lim _{m \rightarrow \infty} \frac{1}{m} \ln \left(\mathscr{L}_{\tilde{f}}^{m}(\mathbf{1})(\underline{x})\right)=\ln \left(\lambda_{f}\right)
$$

uniformemente. 
O número $P(f):=\ln \left(\lambda_{f}\right)$ é dito pressão topológica de $f$. O seguinte resultado a respeito da regularidade da pressão é não trivial

Teorema 1.4.4. A aplicação $C^{\gamma}(\Omega) \ni f \mapsto P(f) \in \mathbb{R}$ é analítica real.

Ou seja para potenciais $f$ Hölder contínuos provenientes de iterações absolutamente somáveis invariantes por translações temos a ausência de transição de fase.

Observamos que os resultados acima, obtidos usando-se o formalismo do operador de Ruelle, são para o látice $\mathbb{N}$ entretanto existem métodos para estender estes resultados para o látice $\mathbb{Z}$, veja por exemplo em [6, 37, 2].

\subsection{AplicaÇÕes do TeOrema de Ruelle-Perron-Fröbenius EM DINÂMICA}

\subsubsection{O Formalismo TermodinÂMico}

A teoria ergódica é uma área dos sistemas dinâmicos que, a grosso modo, visa estudar um sistema dinâmico $T: X \rightarrow X$ do pondo de vista de uma medida $\mu$ invariante. Algumas medidas invariantes tem relações bastante próximas com propriedades da dinâmica, tais como mistura, transitividade e minimalidade. Uma classe bastante ampla de sistemas dinâmicos, que são as dinâmicas contínuas em espaços compactos sempre possuem medidas invariantes, em geral todo um convexo delas.

Exemplo 1.5.1 (Transformação Norte Sul). Considere a projeção estereográfica do círculo $S^{1}=\{z:|z-i|=1\}$ sobre a reta real e defina a aplicação norte sul $T: S^{1} \rightarrow S^{1}$ pondo

$$
T(z)=\left\{\begin{array}{cl}
2 i & \text { se } z=2 i \\
\pi^{-1}\left(\frac{\pi(z)}{2}\right), & \text { se } z \neq 2 i
\end{array}\right.
$$

Usaremos as notações $N=2 i$ e $S=0$, então tanto $N$ quanto $S$ são pontos fixos, e além do mais para todo $z \neq N$ vale $T^{n}(z) \rightarrow S$ quando $n \rightarrow \infty$.

Obviamente, tanto $\delta_{N}$ quanto $\delta_{S}$ são medidas invariantes e por conseguinte também o é qualquer combinação convexa $(1-t) \delta_{N}+t \delta_{S}, t \in(0,1)$.

Então surge naturalmente o problema de se escolher qual a melhor medida invariante para se estudar um sistema dinâmico. Uma primeira resposta, pode ser escolher as medidas ergódicas, pois além do seu caráter indecomponível elas também carregam bastante informação a respeito da estatística das órbitas. Esta também não parece ser uma boa solução como mostra o exemplo abaixo.

Exemplo 1.5.2 (Doubling map). Seja $S^{1}=[0,1] / \sim$, onde $\sim$ é a relação de equivalência que identifica os pontos 0 e 1, equipado com a topologia quociente. Considere então a transformação $T: X \rightarrow X$ dada por $T(x)=2 x(\bmod 1)$. É fácil verificar que qualquer racional diádico em $S^{1}$ é um ponto periódico para $T$. Como também é sabido toda medida suportada numa órbita periódica é invariante e ergódica. 
No exemplo acima todas as medidas suportadas em órbitas periódicas são ergódicas, portanto estas medidas só enxergam o que acontece em cima da órbita periódica em questão. Uma medida ergódica interessante para o doubling map talvez seja a medida de Lebesgue que tem suporte total, de fato pode-se mostrar, através da semiconjugação com o shift que o doubling map tem um contínuo de medida invariantes, ergódicas e de suporte total. Diante do fracasso da tentativa acima, um outro caminho seria tentar escolher uma medida que seja ergódica e que tenha suporte total, mas como mostra o exemplo abaixo estas restrições também não nos deixam em uma situação mais fácil.

Exemplo 1.5.3. Seja $m \in \mathbb{N}$ considere o espaço das sequências $\Sigma_{+}=\{0, \cdots, m-1\}^{\mathbb{N}}$ dado um ponto $p=\left(p_{0}, \ldots, p_{m-1}\right) \in \Delta=\left\{\left(t_{0}, \ldots, t_{m-1}\right) \mid \sum t_{i}=1, t_{i}>0\right\}$, no simplexo unitário existe uma única probabilidade $\mu_{p}$ de Borel invariante pelo shift tal que para qualquer cilindro $\left[a_{0}, \ldots, a_{k}\right]$ vale

$$
\mu_{p}\left(\left[a_{0}, \ldots, a_{k}\right]\right)=p_{a_{0}} \ldots p_{a_{k}} .
$$

Essas medidas são chamadas medidas de Bernoulli. Como o leitor pode ter observado temos todo um contínuo de medidas de Bernoulli. Pode-se mostrar que qualquer medida de Bernoulli é ergódica para o shift.

Uma das estratégias dos dinamicistas diante do dilema de escolher qual a melhor medida invariante para se estudar um sistema dinâmico foi pegar emprestadas ideias da mecânica estatística. Assumindo que a melhor medida para se estudar um sistema é aquela que maximiza a entropia do sistema. Definimos a pressão de um sistema dinâmico $T: X \rightarrow X$ associado a um potencial $f: X \rightarrow \mathbb{R}$ como sendo a quantidade

$$
P_{T}(f)=\sup _{\mu \in \mathcal{M}(X)}\left[h(\mu, T)+\int_{X} f d \mu\right],
$$

onde $h(\mu, T)$ é a entropia de Kolmogorov-Sinai de $\mu$. Se uma medida $\mu \in \mathcal{M}(X)$ é tal que que $P_{T}(f)=h(\mu, T)+\int_{X} f d \mu$ então essa medida é dita um estado de equilíbrio para $f$.

Considere $X=M^{\mathbb{N}}$, onde $M=\{1, \ldots, n-1\}, T: X \rightarrow X$ como sendo o shift para esquerda e $f: X \rightarrow \mathbb{R}$ um potencial Hölder contínuo. A medida de probabilidade $m_{f}=h_{f} \nu_{f}$ onde $\nu_{f}$ e $h_{f}$ são dados pelo teorema de Ruelle (enunciado na seção anterior) é chamada de medida de Gibbs associada a $f$.

Corolário 1.5.4. As seguintes afirmações a respeito da medida de Gibbs $m_{f}$ são verdadeiras,

(a) $m_{f}$ é uma medida invariante para o shift $\sigma: X \rightarrow X$;

(b) O par $\left(\sigma, m_{f}\right)$ é misturador (e portanto $m_{f}$ é ergodica);

(c) A medida $m_{f}$ tem suporte total;

(d) $m_{f}$ é um estado de equilíbrio para o potencial $f$.

Demonstração. Ver [6]. 
DinAmicA Uniformemente HiperbólicA.

Definição 1.5.5. Seja $M$ uma variedade Riemanniana de classe $C^{1}, U \subset M$ um aberto $e$ $f: U \rightarrow f(U)$ um difeomorfismo de classe $C^{1}$. Um conjunto $\Lambda \subset M$ é dito hiperbólico quando, existem constantes $C>0$ e $0<\lambda<1$ e famílias de subespaços, $E^{s}(x) \subset T_{x} M$ e $E^{u}(x) \subset T_{x} M$, $x \in \Lambda$, tal que para todo $x \in \Lambda$ vale:

(a) $T_{x} M=E^{s}(x) \oplus E^{u}(x)$;

(b) $\left\|D f_{x}^{n}\left(v^{s}\right)\right\| \leq C \lambda^{n}\left\|v^{s}\right\|$ para todo $v^{s} \in E^{s}(x)$ e todo $n \geq 0$;

(c) $\left\|D f_{x}^{-n}\left(v^{u}\right)\right\| \leq C \lambda^{n}\left\|v^{u}\right\|$ para todo $v^{u} \in E^{u}(x)$ e todo $n \geq 0$;

(d) $D f_{x}\left(E^{s}(x)\right)=E^{s}(f(x))$ e $D f_{x}\left(E^{u}(x)\right)=E^{u}(f(x))$.

Os espaços $E^{s}(x)$ e $E^{u}(x)$ são chamados de espaços estáveis e instáveis respectivamente, e as famílias $\left\{E^{s}(x)\right\}_{x \in \Lambda}$ e $\left\{E^{u}(x)\right\}_{x \in \Lambda}$ são chamadas distribuições instáveis e estáveis respectivamente de $f \mid \Lambda$. Veja que esta definição permite os casos extremos em que $E^{s}(x)=\{0\}$ ou $E^{u}(x)=\{0\}$.

Seja $f: M \rightarrow M$ um difeomorfismo, dado $x \in M$ definimos a variedade estável local de "tamanho" $\varepsilon$ de $x$ como sendo o conjunto

$$
W_{\varepsilon}^{s}(x)=\left\{y \in M: d\left(f^{n}(x), f^{n}(y)\right) \leq \varepsilon, \forall n \geq 0\right\}
$$

analogamente definimos a variedade instável local de "tamanho" $\varepsilon$ de $x$ como sendo o conjunto

$$
W_{\varepsilon}^{s}(x)=\left\{y \in M: d\left(f^{-n}(x), f^{-n}(y)\right) \leq \varepsilon, \forall n \geq 0\right\}
$$

ou seja $W_{\varepsilon}^{s}(x)$ consiste dos pontos que $\varepsilon$-sombreiam a órbita futura de $x$ e $W_{\varepsilon}^{u}(x)$ os que $\varepsilon$-sombreiam a órbita passada de $x$.

Teorema 1.5.6 (Teorema da variedade estável para conjuntos hiperbólicos). Seja $f: M \rightarrow M$ difeomorfismo de classe $C^{1}$ e $\Lambda$ um conjunto hiperbólico de $f$ cuja decomposição é $T_{\Lambda} M=$ $E_{\Lambda}^{s} \oplus E_{\Lambda}^{u}$. Então existem $0<\mu<1$ e $\varepsilon>0$ tais que para cada $x \in \Lambda$

(a) $W_{\varepsilon}^{s}(x)$ é um disco mergulhado, tangente a $E^{s}(x)$ em $x$;

(b) $d\left(f^{n}(x), f^{n}(y)\right) \leq \mu^{n} d(x, y), \quad \forall y \in W_{\varepsilon}^{s}(x)$;

(c) A família de discos $W_{\varepsilon}^{s}(x)$ varia continuamente com $x$ na topologia $C^{r}$.

Proposição 1.5.7. Seja $\Lambda$ um conjunto hiperbólico. Existem $\varepsilon>0$ e $\delta>0$ tais que para quaisquer dois pontos $x, y \in \Lambda$, com $d(x, y)<\delta$ implica que $W_{\varepsilon}^{s}(x) \cap W_{\varepsilon}^{u}(y)$ se intersectam transversalmente em um único ponto. 
Quando for possível escolher um par adaptado $(\varepsilon, \delta)$ no teorema acima tais que para quaisquer dois pontos $x, y \in \Lambda$ a intersecção $[x, y]:=W_{\varepsilon}^{s}(x) \cap W_{\varepsilon}^{u}(y) \in \Lambda$ diremos que $\Lambda$ tem estrutura de produto local. A motivação para o nome, estrutura de produto local, vem do seguinte, fixe $p \in \Lambda$, podemos escolher $\varepsilon$ suficientemente pequeno tal que a função

$$
\begin{array}{ccc}
h:\left(W_{\varepsilon}^{u}(p) \cap \Lambda\right) \times\left(W_{\varepsilon}^{u}(p) \cap \Lambda\right) & \longrightarrow & \Lambda \\
(x, y) & \longmapsto h(x, y)=[x, y]
\end{array}
$$

seja um homeomorfismo sobre sua imagem.

Teorema 1.5.8 (Decomposição espectral). Seja $f: M \rightarrow M$ um difeomorfismo de classe $C^{1}$ de uma variedade compacta. Assuma que $\overline{\operatorname{Per}(f)}$ tem estrutura hiperbólica. Então existe um número finito de conjuntos $\Lambda_{1}, \ldots, \Lambda_{n}$ tal que

(a) $\overline{\operatorname{Per}(f)}=\Lambda_{1} \cup \ldots \cup \Lambda_{n}$, onde cada $\Lambda_{i}$ é compacto invariante e os pontos periódicos de $f$ são densos em cada $\Lambda_{i}$;

(b) $f \mid \Lambda_{i}$ é transitiva para cada $\Lambda_{i}$;

(c) Cada $\Lambda_{i}$ tem estrutura de produto local.

Cada conjunto $\Lambda_{i}$ na decomposição acima é dito um conjunto básico ou conjunto espectral.

Seja $\Lambda_{j}$ um conjunto básico associado ao teorema acima. Um subconjunto $R \subset \Lambda_{j}$ é dito um retângulo se tem diâmetro pequeno o suficiente para que $[x, y] \in R$ sempre que $x, y \in R$. Um retângulo $R$ é próprio se é fechado e $R=\overline{\operatorname{int} R}$. Seja $x \in R$ então definimos

$$
W^{s}(x, R)=W_{\varepsilon}^{s}(x) \cap R \text { e } W^{u}(x, R)=W_{\varepsilon}^{u}(x) \cap R
$$

onde $\varepsilon>0$ é pequeno o suficiente e o diâmetro de $R$ é pequeno comparado a $\varepsilon$.

Definição 1.5.9. Uma partição de Markov de um conjunto básico $\Lambda_{j}$ é uma cobertura finita $\mathscr{R}=\left\{R_{1}, \ldots, R_{m}\right\}$ de $\Lambda_{j}$ por retângulos próprios tais que as seguintes condições são satisfeitas:

(a) $\operatorname{int} R_{i} \cap \operatorname{int} R_{j}=\varnothing$ para $i \neq j$;

(b) $f\left(W^{u}\left(x, R_{i}\right)\right) \supset W^{u}\left(f(x), R_{j}\right)$ e $f\left(W^{s}\left(x, R_{j}\right)\right) \subset W^{s}\left(f(x), R_{j}\right)$ onde $x \in R_{i}$ e $f(x) \in R_{j}$.

Teorema 1.5.10 (Partições de Markov). Seja $\Lambda_{j}$ um conjunto básico para um difeomorfismo f. Então, $\Lambda_{j}$ possui partições de Markov $\mathscr{R}$ de diâmetro arbitrariamente pequeno.

Dada uma partição de Markov $\mathscr{R}=\left\{R_{1}, \ldots, R_{m}\right\}$ temos uma maneira natural de associar a dinâmica $f$ a uma dinâmica simbólica. De fato considere a matriz de transição $B=\left(b_{i j}\right)$ definida por

$$
b_{i j}= \begin{cases}1, & \text { se } \operatorname{int}\left(f\left(R_{i}\right)\right) \cap \operatorname{int}\left(f\left(R_{j}\right)\right) \neq \varnothing, \\ 0, & \text { se } \operatorname{int}\left(f\left(R_{i}\right)\right) \cap \operatorname{int}\left(f\left(R_{j}\right)\right)=\varnothing .\end{cases}
$$

Considere o subshift $\Sigma_{B}$ associado a $B$ definido por

$$
\Sigma_{B}=\left\{\underline{x}: \mathbb{Z} \rightarrow\{1, \ldots, n\}: b_{x_{i} x_{i+1}}=1\right\},
$$

e $\sigma_{B}$ o shift à esquerda agindo em $\Sigma_{B}$. Então temos o seguinte teorema

Teorema 1.5.11. Para cada $\underline{x} \in \Sigma_{B}$ o conjunto $\cap_{i \in \mathbb{Z}} f^{-i}\left(R_{x_{i}}\right)$ consiste de um único ponto, denotado por $\pi(\underline{x})$. Além do mais o mapa $\pi: \Sigma_{B} \rightarrow \Lambda_{j}$ é uma sobrejeção contínua tal que $\pi \circ \sigma=f \circ \pi$, ou seja $f$ é um fator de $\sigma$. 


\section{Estados de Equilíbrio para Conjuntos BÁsicos}

Teorema 1.5.12. Sejam $\Lambda$ um conjunto básico para um difeomorfismo $f$ e $\varphi: \Lambda \rightarrow \mathbb{R}$ uma aplicação Hölder contínua. Então $\varphi$ tem um único estado de equilíbrio $\mu_{\varphi}$, além do mais $\mu_{\varphi}$ é ergódico e se $f \mid \Lambda$ é mixing então $\varphi$ é Bernoulli.

Embora não pretendamos entrar no mérito de demonstrar o resultado acima vamos contar quem se espera que seja a medida $\mu_{\varphi}$, e o restante serão detalhes técnicos. Suponha sem perda de generalidade que $f \mid \Lambda$ seja mixing, e seja $\mathscr{R}$ uma partição de Markov de $\Lambda$. Então o subshift associado $\sigma_{B}: \Sigma_{B} \rightarrow \Sigma_{B}$ também o é, daí considerando o potencial $\varphi^{\star}=\varphi \circ \pi$ temos, aplicando o Teorema de Ruelle a $\sigma_{B}$ uma medida de Gibbs $\mu_{\varphi^{\star}}$. Então o candidato natural a estado de equilíbrio para $f \mid \Lambda$ é o pull back $\mu_{\varphi}=\pi^{*} \mu_{\varphi^{\star}}$. Uma pergunta natural é a seguinte: qual seria o potencial $\varphi$ mais adequado para se associar à dinâmica $f \mid \Lambda$ ? Talvez a informação numérica que mais carregue informação a respeito da dinâmica hiperbólica $f \mid \Lambda$ sejam as suas taxas de contração e expansão, neste sentido é natural considerarmos os potenciais $\varphi^{(u)}$ e $\varphi^{(s)}$ dados respectivamente por

$$
\varphi^{(u)}(x)=-\log \left\|D f_{x} \mid E_{x}^{u}\right\| \text { e } \varphi^{(s)}(x)=-\log \left\|D f_{x} \mid E_{x}^{s}\right\| .
$$

Estes potenciais carregam consigo bastante informação a respeito da dinâmica $f$, a título de exemplo temos o seguinte teorema:

Teorema 1.5.13. Se $\Lambda$ é um conjunto básico de classe $C^{2}$ então $\varphi^{(u)}: \Lambda \rightarrow \mathbb{R}$ é Hölder continua e as seguintes afirmações são equivalentes,

(a) $\Lambda$ é um atrator;

(b) $m\left(W^{s}(\Lambda)\right)>0$;

(c) $P_{f \mid \Lambda}\left(\varphi^{(u)}\right)=0$. 


\section{Capítulo 2}

\section{ESPAÇOS DE ESTADOS COMPACTOS}

Neste capítulo apresentaremos o operador de Ruelle em um novo contexto, agora ele agirá sobre subespaços das funções contínuas de $\Omega=M^{\mathbb{N}}$, onde $M$ é um espaço métrico compacto. Os subespaços de nosso interesse nesta tese serão, as funções contínuas como um todo, as funções $\gamma$-Hölder contínuas e o Espaço de Walters. Neste capítulo introdutório focaremos nossa atenção apenas em $C(\Omega)$ e $C^{\gamma}(\Omega)$. Em todo este o capítulo consideraremos funções tomando valores em $\mathbb{C}$. Entretanto resultados idênticos valem se substituirmos $\mathbb{C}$ por $\mathbb{R}$.

\subsection{O ESPAÇO $M$ E MEDIDAS A PRIORI}

Na maior parte de nossa exposição $M$ será um espaço métrico compacto. Em geral sempre suporemos que $M$ está equipado com uma medida de probabilidade Boreliana $\mu: \mathscr{B}(M) \rightarrow$ $[0,1]$. Esta medida, que desempenha um papel de extrema importância neste novo contexto, será chamada de medida a priori. Em [1], na Seção 1, uma exposição similar é feita quando $M=S^{1}$ e $\mu$ é a medida de Lebesgue em $S^{1}$. Em, [44] e [32] espaços compactos mais gerais são considerados.

\subsection{O ESPAÇO DAS SEQUENNCIAS}

Seja $(M, d)$ um espaço métrico compacto, no que segue usaremos $\Omega$ para denotar o espaço $\Omega=M^{\mathbb{N}}$ o das sequências infinitas de termos de $M$. Consideramos em $\Omega$ a topologia produto. Munido desta topologia temos do Teorema de Tychonov que $\Omega$ é um espaço topológico compacto. Vamos considerar em $\Omega$ a métrica $d_{\Omega}: \Omega \times \Omega \rightarrow \mathbb{R}$ definida por

$$
d_{\Omega}(x, y)=\sum_{n=1}^{\infty} \frac{d\left(x_{i}, y_{i}\right)}{2^{i}} .
$$

A topologia produto em $\Omega$ é compatível com a métrica $d_{\Omega}$ acima. Ao leitor interessado nesses detalhes técnicos recomendamos a leitura seçao 6 do Capítulo 5 de [31]. 
Algumas CONSIDERAÇÕes A RESPEITO DA MÉTRICA $d_{\Omega}$

O Leitor que está acostumado a trabalhar em $\Omega$ quando $M$ é finito pode se perguntar se poderíamos considerar, ao invés de $d_{\Omega}$, a métrica $d_{\lambda}: M \times M \rightarrow \mathbb{R}$ definida por

$$
d_{\lambda}(x, y)=\lambda^{n_{0}}, \quad n_{0}=\min \left\{n \in \mathbb{N} ; x_{i} \neq y_{i}\right\}, \quad 0<\lambda<1
$$

que tem um aspecto mais simples, mais intuitivo e que quando $M$ é finito é equivalente a $d_{\Omega}$. Entretanto quando $M$ é um compacto arbitrário nem sempre $d_{\Omega}$ e $d_{\lambda}$ são equivalentes, como mostra o seguinte exemplo: considere $M=[0,1]$ como a métrica induzida pelo valor absoluto. Considere, por exemplo, $\lambda=1 / 2$, e o aberto $B_{d_{\lambda}}(\underline{0}, 1 / 2)$ da topologia induzida por $d_{\lambda}$. Temos que

$$
B_{d_{\lambda}}(\underline{0}, 1 / 2)=\{0\} \times[0,1] \times \cdots \times[0,1] \times \cdots
$$

que não é um aberto na topologia induzida por $d_{\Omega}$ (que coincide com a topologia produto).

\subsubsection{Os EspaÇOS $C(\Omega)$ E $C^{\gamma}(\Omega)$}

Denote por $C(\Omega)=C(\Omega, \mathbb{C})$ o espaço das funções a valores complexos que são contínuas em $\Omega$; um elemento típico $f: \Omega \rightarrow \mathbb{C}$, de $C(\Omega)$, é comumente chamado de potencial. Consideraremos em $C(\Omega)$ a norma do máximo, isto é,

$$
\|f\|_{0}=\max _{x \in \Omega}|f(x)| .
$$

É conhecido que $\left(C(\Omega),\|\cdot\|_{0}\right)$ é um espaço de Banach. Outro espaço importante em nossa exposição é o espaço, $C^{\gamma}(\Omega)=C^{\gamma}(\Omega, \mathbb{C})$ das funções $\gamma$-Hölder, mais explicitamente a subclasse das funções $f \in C(\Omega)$ tais que

$$
\operatorname{Hol}(f):=\sup _{x, y \in \Omega} \frac{|f(x)-f(y)|}{d(x, y)^{\gamma}}<\infty .
$$

Vamos trabahar em $C^{\gamma}(\Omega)$ com a norma $\|\cdot\|_{\gamma}$, definida para cada $f \in C^{\gamma}(\Omega)$ por

$$
\|f\|_{\gamma}=\|f\|_{0}+\operatorname{Hol}(f) \text {. }
$$

Abaixo mostramos que $\left(C^{\gamma}(\Omega),\|\cdot\|_{\gamma}\right)$ é um espaço de Banach.

Proposição 2.2.1. O conjunto $N=\left\{f \in C^{\gamma}(\Omega) ;\|f\|_{0} \leq 1,\|f\|_{\gamma} \leq 1\right\}$ é compacto com relação à norma $\|\cdot\|_{0}$.

Demonstração. Pode ser provado pela definição que $N$ é $\|\cdot\|_{0}$-fechado, uma vez observado isso basta mostramos que $N$ é pré-compacto e isto encerra o argumento. Pois bem, para cada $f \in N$ temos $\forall x, y \in \Omega$ que

$$
|f(x)-f(y)| \leq \operatorname{Hol}(f) \cdot d(x, y)^{\gamma},
$$

segue de 2.1) que $N$ é uma família uniformemente equicontínua de funções. Além disso para cada $x \in \Omega$ o conjunto $E_{x}=\{f(x), f \in N\}$ está contido no intervalo $[-1,1]$ sendo assim $E_{x}$ tem fecho compacto. Estamos então em condições de usar o Teorema de Arzelá-Áscoli e garantir que $N$ é pré-compacto, como $N$ é fechado temos que $N=\bar{N}$ é $\|\cdot\|_{0^{-}}$compacto. 
Note que homotetias de $C(\Omega)$, isto é, transformações $M_{\alpha}: C(\Omega) \rightarrow C(\Omega)$ definidas por $M_{\alpha} f=\alpha f, \quad \alpha \in \mathbb{R}$, são aplicações contínuas. Portanto $\forall \alpha$ o conjunto $M_{\alpha} N$ é $\|\cdot\|_{0}$-compacto. Segue facilmente desta observação que $\left(C^{\gamma}(\Omega),\|\cdot\|_{\gamma}\right)$ é um espaço de Banach.

Proposição 2.2.2. $C^{\gamma}(\Omega)$ é uniformemente denso em $C(\Omega)$.

Demonstração. Note que se $d_{\Omega}$ é uma métrica em $\Omega$ então $d_{\Omega}^{\gamma}$, para qualquer $0 \leq \gamma \leq 1$ também é uma métrica em $\Omega$. Para ver isso é suficiente mostrarmos que $(a+b)^{\gamma} \leq a^{\gamma}+b^{\gamma}$ para quaisquer $a, b>0$, ou equivalentemente que,

$$
1 \leq\left(\frac{a}{a+b}\right)^{\gamma}+\left(\frac{b}{a+b}\right)^{\gamma} .
$$

Como $0 \leq \gamma \leq 1$, temos que

$$
\left(\frac{a}{a+b}\right)^{\gamma} \geq \frac{a}{a+b} \text { e }\left(\frac{b}{a+b}\right)^{\gamma} \geq \frac{b}{a+b}
$$

somando as duas desigualdades acima obtemos o desejado. Portanto reduzimos nosso problema a provar que o espaço das funções Lipschitz é uniformemente denso em $C(\Omega)$. Denote por $\operatorname{Lip}(\Omega)$ o subespaço de $C(\Omega)$ constituído pelas funções Lipschitz, note que Lip $(\Omega)$ é uma álgebra de funções que contém as constantes, se mostrarmos que $\operatorname{Lip}(\Omega)$ separa pontos a proposição seguira diretamente do teorema de Stone Weierstrass. Para ver que $\operatorname{Lip}(\Omega)$ separa pontos vamos usar o fato de que a métrica $d_{\Omega}$ é Lipschitz: sejam $x_{0}, y_{0} \in \Omega$, considere a função $f: \Omega \rightarrow \mathbb{R}$ dada por $f(y)=d_{\Omega}\left(x_{0}, y\right)$, é claro que $f\left(y_{0}\right)>0=f\left(x_{0}\right)$, note que $f$ é Lipschitz, visto que

$$
|f(y)-f(x)|=\left|d_{\Omega}\left(y, x_{0}\right)-d_{\Omega}\left(x_{0}, x\right)\right| \leq d_{\Omega}(x, y) .
$$

Portanto segue o resultado.

Proposição 2.2.3. Os espaços $C(\Omega)$ e $C^{\gamma}(\Omega)$ são álgebras de Banach, em outras palavras valem as seguintes desigualdades,

(a) $\|f g\|_{0} \leq\|f\|_{0} \cdot\|g\|_{0} \quad \forall f, g \in C(\Omega)$;

(b) $\|f g\|_{\gamma} \leq\|f\|_{\gamma} \cdot\|g\|_{\gamma} \forall f, g \in C^{\gamma}(\Omega)$.

Demonstração. A prova do item (a) é trivial e a omitiremos. Para a prova de $(b)$ veja que para todos $x, y \in \Omega$ temos que

$$
\begin{aligned}
\mid(f g)(x)-f g)(y) \mid & \leq|f(x) g(x)-f(x) g(y)|+|f(x) g(y)-f(y) g(y)| \\
& \leq|f(x)||g(x)-g(y)|+|g(y)||f(x)-f(y)| \\
& \leq\|f\|_{0} \operatorname{Hol}(g) d(x, y)^{\gamma}+\|g\|_{0} \operatorname{Hol}(f) d(x, y)^{\gamma} .
\end{aligned}
$$

Portanto se $x \neq y$ temos que

$$
\frac{|(f g)(x)-(f g)(y)|}{d(x, y)^{\gamma}} \leq\|f\|_{0} \operatorname{Hol}(g)+\|g\|_{0} \operatorname{Hol}(f)
$$

e como $\|f g\|_{0} \leq\|f\|_{0} \cdot\|g\|_{0}$ segue da desigualdade acima que $\|f g\|_{\gamma} \leq\|f\|_{\gamma} \cdot\|g\|_{\gamma}$. 


\section{O ESPAÇO DOS OPERADORES LINEARES E DAS FORMAS MULTILINEARES}

Seja $\mathcal{K} \subset C(\Omega)$ um subespaço equipado com uma norma $\|\cdot\|$ de tal modo que o par $(C(\Omega,\|\cdot\|)$ seja um espaço de Banach. Denote por $V=\mathcal{L}(\mathcal{K}, \mathcal{K})$ o espaço dos operadores lineares limitados de $\mathcal{K}$ em $\mathcal{K}$. Dado um operador $l \in \mathcal{L}(\mathcal{K}, \mathcal{K})$ definimos a norma $|l|_{V}$ de $l$ como

$$
|l|_{V}=\sup _{0 \neq \varphi \in \mathcal{K}} \frac{\|l(\varphi)\|}{\|\varphi\|} .
$$

Lembramos que $|l|_{V}$ também pode ser calculado do seguinte modo, $|l|_{V}=\sup _{\|\varphi\|=1}\|l(\varphi)\|$. Claramente o espaço normado $\left(\mathcal{L}(V),|\cdot|_{V}\right)$ é um espaço de Banach.

\subsection{O OPERADOR DE RuElle}

Antes de apresentarmos o operador de Ruelle vamos fixar mais uma notação. Dados $n \in \mathbb{N}$, $\left(a_{1}, a_{2}, \ldots, a_{n}\right) \in M^{n}$ e $x \in \Omega$ vamos usar a notação $a_{1} a_{2} \ldots a_{n} x$ para representar uma sequência $y \in \Omega$ cujas as coordenadas são dadas por $y_{1}=a_{1}, y_{2}=a_{2}, \ldots, y_{n}=a_{n}, y_{n+1}=x_{1}, y_{n+2}=$ $x_{2}, \ldots$ Em outras palavras a sequência representada por $a_{1} a_{2} \ldots a_{n} x$ é a concatenação da palavra $\left(a_{1}, a_{2}, \ldots, a_{n}\right) \in M^{n}$ com a sequência $x \in \Omega$.

Fixada uma medida apriori $\mu$ com as propriedades descritas no inicio desta seção e dado $f \in C(\Omega)$ definimos o operador $\mathscr{L}_{f}: C(\Omega) \rightarrow C(\Omega)$ pondo para cada $\varphi \in C(\Omega)$,

$$
\left(\mathscr{L}_{f} \varphi\right)(x)=\int_{M} \varphi(a x) e^{f(a x)} d \mu(a),
$$

onde a notação ax, como definida acima, representa o elemento do espaço $\Omega$ cujas as coordenadas são dadas por $\left(a, x_{1}, x_{2}, \ldots\right)$. A seguir listamos algumas propriedades elementares do operador de Ruelle que serão bastante úteis no decorrer do texto.

Propriedade 1. Definindo $S_{n} f(y)=\sum_{j=0}^{n-1} f \circ \sigma^{j}(y)$ então podemos ver que a composição sucessiva $n$-vezes do operador $\mathscr{L}_{f}$ é dado pela seguinte expressão

$$
\begin{aligned}
\mathscr{L}_{f}^{n} \varphi(x) & =\int_{M^{n}} \varphi\left(a_{1} a_{2} \ldots a_{n} x\right) e^{S_{n} f\left(a_{1} a_{2} \ldots a_{n} x\right)} d \mu\left(a_{1}\right) \ldots d \mu\left(a_{n}\right) \\
& \equiv \int_{\mathbf{a} \in M^{n}} \varphi(\mathbf{a} x) e^{S_{n} f(\mathbf{a} x)} d \mu(\mathbf{a}) .
\end{aligned}
$$

Na notação introduzida depois do sinal "三” há um pequeno abuso. Já que a integral é calculada com respeito a medida produto de $n$-cópias da medida a-priori $\mu$.

Propriedade 2. Sejam $\varphi \in C(\Omega)$ e $\varphi \circ \sigma$ sua composição com o shift. Então temos que

$$
\mathscr{L}_{f}(\varphi \circ \sigma)(x)=\int_{a \in M} \varphi \circ \sigma(a x) e^{f(a x)} d \mu(a)=\int_{a \in M} \varphi(x) e^{f(a x)} d \mu(a)=\varphi(x) \mathscr{L}_{f} \mathbf{1}(x) .
$$

Propriedade 3. Devido a compacidade de $\Omega$ em relação à topologia produto e ao Teorema de Riez Markov temos que $(C(\Omega))^{*}$ é isometricamente isomorfo a $\mathcal{M}_{s}(\Omega)$ (medidas borelianas 
sinaladas munido da norma da variação total). Portanto podemos definir o adjunto do operador de Ruelle (ou o operador de Ruelle dual) como sendo o único mapa de $\mathcal{M}_{s}(\Omega)$ em $\mathcal{M}_{s}(\Omega)$ contínuo satisfazendo, para cada $\nu \in \mathcal{M}_{s}(\Omega)$ a seguinte identidade

$$
\int_{\Omega} \mathscr{L}_{f} \varphi d \nu=\int_{\Omega} \varphi d\left[\mathscr{L}^{*} \nu\right] \quad \forall \varphi \in C(\Omega) .
$$

Para verificar esta igualdade, fixe uma medida de Borel $\nu$ finita e considere o funcional linear $F_{\nu}: C(\Omega) \rightarrow \mathbb{R}$ dado por

$$
F_{\nu}(\varphi)=\int_{\Omega} \mathscr{L}_{f} \varphi d \nu
$$

Claramente $F_{\nu}$ é um funcional linear e além do mais é contínuo em $\left(C(\Omega),\|\cdot\|_{0}\right)$, pois $\mathscr{L}_{f}$ é um operador positivo e vale a seguinte desigualdade

$$
\left|F_{\nu}(\varphi)\right| \leq \int_{\Omega}\left|\mathscr{L}_{f} \varphi\right| d \nu \leq \int_{\Omega}\|\varphi\|_{0}\left|\mathscr{L}_{f} \mathbf{1}\right| d \nu \leq\left[\left\|\mathscr{L}_{f} \mathbf{1}\right\|_{0} \cdot \nu(\Omega)\right] \cdot\|\varphi\|_{0} .
$$

Da positividade de $\mathscr{L}_{f}$ segue que $F_{\nu}$ é um funcional linear positivo. Portanto pelo Teorema de Riesz-Markov sabemos que existe uma única $\gamma_{\nu}$ medida de Borel finita sobre $\Omega$ tal que para toda $\varphi \in C(\Omega)$ temos

$$
F_{\nu}(\varphi)=\int_{\Omega} \varphi d \gamma_{\nu} \quad \text { ou seja } \quad \int_{\Omega} \mathscr{L}_{f} \varphi d \nu=\int_{\Omega} \varphi d \gamma_{\nu}
$$

Afirmamos que $\gamma_{\nu}=\mathscr{L}_{f}^{*}(\nu)$.

Antes de provar esta afirmação vamos recordar a definição de operador dual (ou adjunto) em espaços de Banach. Seja $\mathcal{B}$ um espaço de Banach e $T: \mathcal{B} \rightarrow \mathcal{B}$ um operador linear limitado. O dual ou adjunto de $T$ denotado por $T^{*}$ é definido como sendo o único operador $T^{*}: \mathcal{B}^{*} \rightarrow \mathcal{B}^{*}$ satisfazendo

$$
F(T(x))=T^{*}(F)(x) \quad \forall F \in \mathcal{B}^{*} \text { e } \forall x \in \mathcal{B} .
$$

Voltamos a prova da afirmação. Vamos tomar na definição acima $\mathcal{B}=C(\Omega)$ e $T=\mathscr{L}_{f}$. Como mencionado acima $(C(\Omega))^{*}$ é isometricamente isomorfo a $\mathcal{M}_{s}(\Omega)$. Com esta identificação do dual de $C(\Omega)$ a definição de $\nu(\varphi)$ para $\nu \in \mathcal{M}_{s}(\Omega)$ e $\varphi \in C(\Omega)$ é feita da seguinte maneira

$$
\nu(\varphi) \equiv \int_{\Omega} \varphi d \nu .
$$

Vamos pensar no operador dual $\mathscr{L}_{f}^{*}$ como um operador de $\mathcal{M}_{s}(\Omega)$ em $\mathcal{M}_{s}(\Omega)$. Usando a expressão acima temos para todo $\nu \in \mathcal{M}_{s}(\Omega), \varphi \in C(\Omega)$ que

$$
\mathscr{L}_{f}^{*}(\nu)(\varphi)=\int_{\Omega} \varphi d\left[\mathscr{L}_{f}^{*}(\nu)\right]
$$

Usando (2.2) e a definição de operador dual (ou adjunto) temos

$$
\int_{\Omega} \varphi d \gamma_{\nu}=\int_{\Omega} \mathscr{L}_{f} \varphi d \nu=\nu\left(\mathscr{L}_{f} \varphi\right)=\mathscr{L}_{f}^{*}(\nu)(\varphi)=\int_{\Omega} \varphi d\left[\mathscr{L}^{*} \nu\right] \quad \forall \varphi \in C(\Omega) .
$$


Como a igualdade acima é válida para toda função contínua $\varphi$ segue que $\gamma_{\nu}=\mathscr{L}^{*} \nu$ como queríamos demonstrar.

Antes de prosseguirmos perguntas naturais surgem, a primeira é se este operador está bem definido. Uma outra pergunta natural, tendo em vista os resultados já conhecidos para o caso $\Omega=\{0,1\}^{\mathbb{N}}$, é se o operador de Ruelle deixa invariante o espaço dos potenciais Hölder.

Proposição 2.3.1. Para qualquer $f \in C^{\gamma}(\Omega), 0 \leq \gamma \leq 1$, o operador de Ruelle $\mathscr{L}_{f}: C^{\gamma}(\Omega) \rightarrow$ $C^{\gamma}(\Omega)$ associado a $f$ está bem definido e é um operador linear limitado. Mais precisamente temos que

(a) Se $f \in C(\Omega)$ então $\mathscr{L}_{f} \varphi \in C(\Omega) \forall \varphi \in C(\Omega)$;

(b) Se $f \in C^{\gamma}(\Omega), 0<\gamma \leq 1$ então $\mathscr{L}_{f} \varphi \in C^{\gamma}(\Omega) \forall \varphi \in C^{\gamma}(\Omega)$;

(c) Se $f \in C(\Omega)$ então $\mathscr{L}_{f}: C(\Omega) \rightarrow C(\Omega)$ é um operador linear limitado;

(d) Se $f \in C^{\gamma}(\Omega), 0<\gamma \leq 1$, então $\mathscr{L}_{f}: C^{\gamma}(\Omega) \rightarrow C^{\gamma}(\Omega)$ é um operador linear limitado.

Demonstração. Prova do item (a). Para todos $x$ e $y$ em $\Omega$, temos,

$$
\begin{aligned}
\left|\mathscr{L}_{f} \varphi(x)-\mathscr{L}_{f} \varphi(y)\right| & =\left|\int_{M} e^{f(a x)} \varphi(a x) d \mu(a)-\int_{M} e^{f(a y)} \varphi(a y) d \mu(a)\right| \\
& \leq \int_{M}\left|e^{f(a x)} \varphi(a x)-e^{f(a y)} \varphi(a y)\right| d \mu(a) .
\end{aligned}
$$

Estimando o integrando acima obtemos,

$$
\begin{aligned}
\left|e^{f(a x)} \varphi(a x)-e^{f(a y)} \varphi(a y)\right| & =\left|e^{f(a x)} \varphi(a x)-e^{f(a x)} \varphi(a y)+e^{f(a x)} \varphi(a y)-e^{f(a y)} \varphi(a y)\right| \\
& \leq\left|e^{f(a x)}(\varphi(a x)-\varphi(a y))\right|+\left|\varphi(a y)\left(e^{f(a x)}-e^{f(a y)}\right)\right|
\end{aligned}
$$

Temos pelo Teorema do Valor Intermediário que $e^{z}-e^{w}=e^{\hat{z}}(z-w)$, para todo $z$ e $w$ em $\mathbb{R}$ e $\operatorname{algum} \hat{z}$ entre $z$ e $w$. Portanto, $\left|e^{f(a x)}-e^{f(a y)}\right| \leq e^{\|f\|_{0}}|f(a x)-f(a y)|$ e logo

$$
\left|e^{f(a x)} \varphi(a x)-e^{f(a y)} \varphi(a y)\right| \leq e^{\|f\|_{0}}\left(|\varphi(a x)-\varphi(a y)|+\|\varphi\|_{0}|f(a x)-f(a y)|\right) .
$$

Agora, fixe $\varepsilon>0$. Como $\Omega$ é compacto $\varphi$ e $f$ são uniformemente contínuas. Portanto, existe $\delta_{1}>0$ tal que

$$
\max \{|\varphi(t)-\varphi(s)|,|f(t)-f(s)|\}<\frac{\varepsilon}{\mu(M) e^{\|f\|_{0}\left(1+\|\varphi\|_{0}\right)}}, \text { se } d_{\Omega}(t, s)<\delta_{1}, t, s \in \Omega .
$$

Usando $d_{\Omega}(a x, a y)=\frac{d_{\Omega}(x, y)}{2}$ e $(2.3)$, se $d_{\Omega}(x, y)<2 \delta_{1}$ temos que

$$
\left|\mathscr{L}_{f} \varphi(x)-\mathscr{L}_{f} \varphi(y)\right|<\int_{M} e^{\|f\|_{0}}\left\{\frac{\varepsilon}{\mu(M) e^{\|f\|_{0}}\left(1+\|\varphi\|_{0}\right)}+\|\varphi\|_{0} \frac{\varepsilon}{\mu(M) e^{\|f\|_{0}}\left(1+\|\varphi\|_{0}\right)}\right\} d \mu(a)=\varepsilon
$$


o que implica que $\mathscr{L}_{f} \varphi: \Omega \rightarrow \mathbb{R}$ é uma função uniformemente contínua, em particular $\mathscr{L}_{f} \varphi \in$ $C(\Omega)$.

Prova do item $(b)$. De $(2.3)$ temos que

$$
\left|e^{f(a x)} \varphi(a x)-e^{f(a y)} \varphi(a y)\right| \leq e^{\|f\|_{0}}\left\{\operatorname{Hol}(\varphi) d_{\Omega}^{\gamma}(a x, a y)+\|\varphi\|_{0} \operatorname{Hol}(f) d_{\Omega}^{\gamma}(a x, a y)\right\} .
$$

Lembrando que $d_{\Omega}(a x, a y)=\frac{d(x, y)}{2}$, temos que

$$
\begin{aligned}
\left|\mathscr{L}_{f} \varphi(x)-\mathscr{L}_{f} \varphi(y)\right| & \leq \frac{1}{c^{\gamma}} \int_{M} e^{\|f\|_{0}}\left\{\operatorname{Hol}(\varphi) d_{\Omega}^{\gamma}(x, y)+\|\varphi\|_{0} \operatorname{Hol}(f) d_{\Omega}^{\gamma}(x, y)\right\} d \mu(a) \\
& =\frac{\mu(M) e^{\|f\|_{0}}}{2^{\gamma}}\left\{\operatorname{Hol}(\varphi)+\|\varphi\|_{0} \operatorname{Hol}(f)\right\} d_{\Omega}^{\gamma}(x, y) .
\end{aligned}
$$

Daí

$$
\sup _{x \neq y} \frac{\left|\mathscr{L}_{f} \varphi(x)-\mathscr{L}_{f} \varphi(y)\right|}{d_{\Omega}^{\gamma}(x, y)} \leq \frac{\mu(M) e^{\|f\|_{0}}}{c^{\gamma}}\left\{\operatorname{Hol}(\varphi)+\|\varphi\|_{0} \operatorname{Hol}(f)\right\},
$$

ou seja, $\mathscr{L}_{f} \varphi \in C^{\gamma}(\Omega)$.

Prova do item $(c)$. O operador, $\mathscr{L}_{f}: C(\Omega) \rightarrow C(\Omega)$ é claramente linear. Agora, dado $\varphi \in C(\Omega)$ temos,

$$
\left\|\mathscr{L}_{f}(\varphi)\right\|_{0}=\sup _{x \in \Omega}\left|\int_{M} e^{f(a x)} \varphi(a x) d \mu(a)\right| \leq \mu(M) e^{\|f\|_{0}}\|\varphi\|_{0} .
$$

Portanto, $\mathscr{L}_{f}: C(\Omega) \rightarrow C(\Omega)$ é um operador linear limitado.

Prova do item $(d)$. É obvio que $\mathscr{L}_{f}: C^{\gamma}(\Omega) \rightarrow C^{\gamma}(\Omega)$ é um operador linear. Segue de (2.4) que $\varphi \in C^{\gamma}(\Omega)$ temos,

$$
\begin{aligned}
\operatorname{Hol}\left(\mathscr{L}_{f} \varphi\right) & \leq \frac{\mu(M) e^{\|f\|_{0}}}{2^{\gamma}}\left\{\operatorname{Hol}(\varphi)+\|\varphi\|_{0} \operatorname{Hol}(f)\right\} \\
& \leq \frac{\mu(M) e^{\|f\|_{0}}}{2^{\gamma}}\left\{\|\varphi\|_{\gamma}+\|\varphi\|_{\gamma}\|f\|_{\gamma}\right\} \\
& =\frac{\mu(M) e^{\|f\|_{0}}}{2^{\gamma}}\left(1+\|f\|_{\gamma}\right)\|\varphi\|_{\gamma} .
\end{aligned}
$$

Além disso temos que

$$
\left\|\mathscr{L}_{f} \varphi\right\|_{0} \leq \mu(M) e^{\|f\|_{0}}\|\varphi\|_{0} \leq \mu(M) e^{\|f\|_{0}}\|\varphi\|_{\gamma},
$$

onde a primeira desigualdade foi provada na demonstração do item (c). Daí

$$
\left\|\mathscr{L}_{f} \varphi\right\|_{\gamma}=\left\|\mathscr{L}_{f} \varphi\right\|_{0}+\operatorname{Hol}\left(\mathscr{L}_{f} \varphi\right) \leq\left\{\frac{\mu(M) e^{\|f\|_{0}}}{2^{\gamma}}\left(1+\|f\|_{\gamma}\right)+\mu(M) e^{\|f\|_{0}}\right\}\|\varphi\|_{\gamma}
$$

o que implica que $\mathscr{L}_{f}: C^{\gamma}(\Omega) \rightarrow C^{\gamma}(\Omega)$ é um operador linear limitado. 


\subsubsection{O Teorema de Ruelle-Perron-Fröbenius (RPF)}

Nesta seção vamos mostrar o resultado principal deste capítulo. Vamos seguir o roteiro dado em [37] que fornece uma demonstração relativamente simples do teorema de RPF. A mudança de contexto, onde o espaço de estados deixa de ser $\{0,1\}$ para ser um espaço métrico compacto $M$ qualquer, trará uma ou outra modificação na prova, mas nada que modifique estruturalmente seu roteiro.

Definição 2.3.2. Diremos que um potencial real $f \in C(\Omega)$ é normalizado se $\mathscr{L}_{f} \mathbf{1}=\mathbf{1}$ onde 1 é a função constante igual a 1.

O seguinte lema tem um papel fundamental na demonstração do teorema de Ruelle Perron Föbenius, que será enunciado posteriormente.

Lema 2.3.3 (Lema Principal). Seja $f=u+i v \in C^{\gamma}(\Omega)$ um potencial e considere o operador de Ruelle associado $\mathscr{L}_{f}: C^{\gamma}(\Omega) \rightarrow C^{\gamma}(\Omega)$, se u é normalizado então para toda $\varphi \in C^{\gamma}(\Omega)$ temos

$$
\operatorname{Hol}\left(\mathscr{L}_{f}^{n} \varphi\right) \leq C\|\varphi\|_{0}+\frac{1}{2^{n \gamma}} \operatorname{Hol}(\varphi) .
$$

Demonstração.

$$
\begin{aligned}
\left|\mathscr{L}_{f}(x)-\mathscr{L}_{f}(y)\right| & =\left|\int_{a \in M} \varphi(a x) e^{f(a x)} d \mu(a)-\int_{a \in M} \varphi(a y) e^{f(a y)} d \mu(a)\right| \\
& \leq \int_{a \in M}\left|\varphi(a x) e^{f(a x)}-\varphi(a x) e^{f(a y)}+\varphi(a x) e^{f(a y)}-\varphi(a y) e^{f(a y)}\right| d \mu(a) \\
& \leq \int_{a \in M}\left|\varphi(a x)\left(e^{f(a x)}-e^{f(a y)}\right)\right| d \mu(a)+\int_{a \in M}\left|e^{f(a y)}(\varphi(a x)-\varphi(a y))\right| d \mu(a) \\
& \leq\|\varphi\|_{0} \int_{a \in M}\left|e^{f(a x)}-e^{f(a y)}\right| d \mu(a)+\operatorname{Hol}(\varphi) d(a x, a y)^{\gamma} \underbrace{\int_{a \in M}\left|e^{f(a y)}\right| d \mu(a)}_{\text {pot. normalizado }} \\
& \leq\|\varphi\|_{0} \cdot C_{1} \int_{a \in M}|f(a x)-f(a y)| d \mu(a)+\operatorname{Hol}(\varphi) d(a x, a y)^{\gamma} \\
& \leq C_{1} \operatorname{Hol}(f) d(a x, a y)^{\gamma}\|\varphi\|_{0}+\operatorname{Hol}(\varphi) d(a x, a y)^{\gamma}
\end{aligned}
$$

como $d(a x, a y)^{\gamma}=\left(1 / 2^{\gamma}\right) \cdot d(x, y)^{\gamma}$ ( a constante $C_{1}$ é dada pelo teorema do valor médio) temos que

$$
\begin{aligned}
\frac{\left|\mathscr{L}_{f}(x)-\mathscr{L}_{f}(y)\right|}{d(x, y)^{\gamma}} & \leq \underbrace{C_{1} \operatorname{Hol}(f) \frac{1}{2^{\gamma}}}_{=C_{0}}\|\varphi\|_{0}+\frac{1}{2^{\gamma}} \operatorname{Hol}(f) \\
& \leq C_{0}\|\varphi\|_{0}+\frac{1}{2^{\gamma}} \operatorname{Hol}(f)
\end{aligned}
$$


donde $\operatorname{Hol}\left(\mathscr{L}_{f} \varphi\right) \leq C_{0}\|\varphi\|_{0}+\left(1 / 2^{\gamma}\right) \cdot \operatorname{Hol}(f)$. O caso geral segue por indução. Com efeito suponha que tenhamos $\operatorname{Hol}\left(\mathscr{L}_{f}^{n} \varphi\right) \leq C_{n}\|\varphi\|_{0}+\left(1 / 2^{n \gamma}\right) \cdot \operatorname{Hol}(\varphi)$ então vem que

$$
\begin{aligned}
\operatorname{Hol}\left(\mathscr{L}_{f}^{n+1} \varphi\right) & =\operatorname{Hol}\left(\mathscr{L}_{f}^{n}\left(\mathscr{L}_{f} \varphi\right)\right) \\
& \left.\leq C_{n}\left\|\mathscr{L}_{f} \varphi\right\|_{0}+\frac{1}{2^{n \gamma}} \operatorname{Hol}\left(\mathscr{L}_{f} \varphi\right)\right) \\
& \leq C_{n}\|\varphi\|_{0}+\frac{1}{2^{n \gamma}}\left(C_{0}\|\varphi\|_{0}+\frac{1}{2^{\gamma}} \operatorname{Hol}(\varphi)\right) \\
& =\underbrace{\left(C_{n}+C_{0} \frac{1}{2^{n \gamma}}\right)}_{:=C_{n+1}}\|\varphi\|_{0}+\frac{1}{2^{(n+1) \gamma}} \operatorname{Hol}(\varphi) .
\end{aligned}
$$

O resultado estará terminado quando encontrarmos um majorante para $C_{n+1}$. Pois bem, veja que $C_{n+1}$ é fornecido indutivamente por $C_{n+1}=C_{n}+C_{0} \frac{1}{2^{n \gamma}}$, sendo assim

$$
C_{n+1}=C_{0}\left(\sum_{k=0}^{n}\left(\frac{1}{2^{\gamma}}\right)^{k}\right)=C_{0} \cdot \frac{1-\frac{1}{2^{(n+1) \gamma}}}{1-\frac{1}{2^{\gamma}}} \leq C_{0} \cdot \frac{1}{1-\left(1 / 2^{\gamma}\right)} .
$$

Finalmente tomando $C=C_{0} \cdot \frac{1}{1-\left(1 / 2^{\gamma}\right)}$ concluímos o resultado.

Teorema 2.3.4 (Ruelle-Perron-Fröbenius). Seja $f \in C^{\gamma}(\Omega, \mathbb{R})$. Então

(a) o operador $\mathscr{L}_{f}: C(\Omega, \mathbb{R}) \rightarrow C(\Omega, \mathbb{R})$ possui um autovalor positivo simples 1 , que denotaremos por $\lambda_{f}$, cuja autofunção associada, $h_{f}$, é positiva e além do mais $h_{f} \in C^{\gamma}(\Omega, \mathbb{R})$;

(b) o espectro do operador $\mathscr{L}_{f}: C^{\gamma}(\Omega, \mathbb{R}) \rightarrow C^{\gamma}(\Omega, \mathbb{R})$ consiste de $\lambda_{f}$ e de um restante contido em um disco de raio estritamente menor que $\lambda_{f}$;

(c) existe uma única probabilidade $\nu_{f}$, definida na $\sigma$-álgebra de Borel de $\Omega$, satisfazendo $\mathscr{L}_{f}^{*} \nu_{f}=\lambda_{f} \nu_{f}$, em outras palavras,

$$
\int_{\Omega} \varphi d\left[\mathscr{L}_{f}^{*} \nu_{f}\right]=\int_{\Omega} \mathscr{L}_{f}^{*} \varphi d \nu_{f}=\lambda_{f} \int_{\Omega} \varphi d \nu_{f} \quad \forall \varphi \in C(\Omega, \mathbb{R}) ;
$$

(d) $\left\|\lambda_{f}^{-n} \mathscr{L}_{f}^{n} \varphi-h_{f} \int_{\Omega} \varphi d \nu_{f}\right\|_{0} \rightarrow 0$ quando $n$ tende a infinito $\forall \varphi \in C(\Omega, \mathbb{R})$.

Prova do item $(a)$. Considere o conjunto

$$
\Lambda_{c}=\left\{g \in C(\Omega, \mathbb{R}): 0 \leq g \leq 1 \text { e } g(x) \leq g(y) \cdot \exp \left(c \cdot d^{\gamma}(x, y)\right)\right\},
$$

onde $c$ é uma constante positiva. O conjunto $\Lambda_{c}$ é convexo e uniformemente fechado, a demonstração disso é trivial e a omitiremos.

\footnotetext{
${ }^{1} \mathrm{O}$ autoespaço associado tem dimensão 1
} 
Note que as desigualdades abaixo mostram que $\Lambda_{c}$ é uma família uniformemente equicontínua. De fato,

$$
\begin{aligned}
|g(x)-g(y)| & \leq|g(y)|\left[\exp \left(c \cdot d(x, y)^{\gamma}\right)-1\right] \\
& \leq\|g\|_{0} \cdot c \cdot e^{c d(x, y)} \cdot d(x, y)^{\gamma} \\
& \leq \text { const } \cdot d(x, y)^{\gamma}
\end{aligned}
$$

onde da primeira para a segunda desigualdade usamos a expansão em serie de Taylor da função exponencial e da segunda para a terceira desigualdade usamos que a função $e^{c d(x, y)^{\gamma}}$ tem valor máximo em $\Omega$ por $\Omega$ ser compacto, provando assim a afirmação. Assim podemos aplicar o Teorema de Arzelá Áscoli e concluir $\Lambda_{c}$ é compacto na norma $\|\cdot\|_{0}$.

Obs. A desigualdade acima mostra também que $\Lambda_{c} \subset C^{\gamma}(\Omega)$.

Defina agora, para cada $n \geq 1$ e para cada $g \in \Lambda_{c}$ o operador $L_{n}$ como segue

$$
L_{n}(g)=\frac{\mathscr{L}_{f}(g+1 / n)}{\left\|\mathscr{L}_{f}(g+1 / n)\right\|_{0}} .
$$

Afirmação. $L_{n}\left(\Lambda_{c}\right) \subset \Lambda_{c}$ para $c$ suficientemente grande. De fato se $g \in \Lambda_{c}$ temos que

$$
\begin{aligned}
\mathscr{L}_{f} g(x) & =\int_{M} g(a x) e^{f(a x)} d \mu(a) \\
& \leq \int_{M} g(a y) e^{f(a x)} e^{c d(a x, a y)^{\gamma}} d \mu(a) \\
& \leq \int_{M} g(a y) e^{f(a y)} e^{c d(a x, a y)^{\gamma}} e^{f(a x)-f(a y)} d \mu(a)
\end{aligned}
$$

como $d(a x, a y)^{\gamma}=\frac{1}{2^{\gamma}} d(x, y)^{\gamma}$ e $|f(a x)-f(a y)| \leq \operatorname{Hol}(f) d(a x, a y)$ temos que

$$
\mathscr{L}_{f} g(x) \leq e^{(c+\operatorname{Hol}(f)) \frac{1}{2^{\gamma}} \cdot d(x, y)^{\gamma}} \mathscr{L}_{f} g(y)
$$

mostramos com isso que $\mathscr{L}_{f} g \in \Lambda_{c_{1}}$ onde $c_{1}=(c+\operatorname{Hol}(f)) \cdot\left(1 / 2^{\gamma}\right)$. Agora por definição temos que se $b<c$ então $\Lambda_{b} \subset \Lambda_{c}$. Então a afirmação estará provada se pudermos escolher um $c$ de tal modo que $c_{1}<c$. Para tanto basta considerarmos a inequação na variável $c$ : $c_{1}=(c+\operatorname{Hol}(f)) \cdot\left(1 / 2^{\gamma}\right)<c$ cuja solução é $c>\left(\operatorname{Hol}(f) \cdot\left(1 / 2^{\gamma}\right)\right) /\left(1-1 / 2^{\gamma}\right)$. Sendo assim para $c$ suficientemente grande temos que $\mathscr{L}_{f}\left(\Lambda_{c}\right) \subset \Lambda_{c_{1}} \subset \Lambda_{c}$. Note que para cada $n$ a função constante $(1 / n) \cdot \mathbf{1}$ pertence a $\Lambda_{c}$ segue então do exposto acima aplicado à função $g+(1 / n) \cdot \mathbf{1} \in \Lambda_{c}$ que

$$
\mathscr{L}_{f}\left(g+\frac{1}{n}\right)(x) \leq e^{c \cdot d(x, y)} \mathscr{L}_{f}\left(g+\frac{1}{n}\right)(y)
$$

dividindo ambos os membros por $\left\|\mathscr{L}_{f}(g+1 / n)\right\|_{0}>0$ provamos a afirmação. Portanto, como $\Lambda_{c}$ é convexo e compacto na norma $\|\cdot\|_{0}$ temos pelo Teorema de Tychonov-Schauder que para cada $n$ existe um ponto fixo $h_{n}$ para $L_{n}$. Sendo assim para cada $n$ temos que

$$
\mathscr{L}_{f}\left(h_{n}+\frac{1}{n}\right)=\beta_{n} h_{n}
$$


onde $\beta_{n}=\left\|\mathscr{L}_{f}(g+1 / n)\right\|_{0}$. Pela compacidade de $\Lambda_{c}$ podemos escolher um ponto de acumulação $h \in \Lambda_{c}$ da sequência $\left\{h_{n}\right\}_{n \in \mathbb{N}}$ e obter por continuidade que $\mathscr{L}_{f} h=\beta h$.

Afirmação 1.: O autovalor $\beta$ é positivo. Para ver isto basta notar que

$$
\beta_{n} h_{n}(x)=\int_{a \in M}\left(h_{n}(a x)+\frac{1}{n}\right) e^{f(a x)} d \mu(a) \geq\left(\inf _{y \in \Omega} h_{n}(y)+\frac{1}{n}\right) e^{-\|f\|_{0}}
$$

dai segue que

$$
\beta_{n} \inf _{x \in \Omega} h_{n}(x) \geq\left(\inf _{y \in \Omega} h_{n}(y)+\frac{1}{n}\right) e^{-\|f\|_{0}} \geq \inf _{y \in \Omega} h_{n}(y) e^{-\|f\|_{0}} .
$$

Portanto dividindo ambos os membros da desigualdade acima por inf $h_{n}$ e passando ao limite em $n$, chegamos a $\beta \geq e^{-\|f\|_{0}}$.

Afirmação 2.: A autofunção $h$ é estritamente positiva. Suponha por contradição que para algum $x \in \Omega$ tenhamos $h(x)=0$, então para todo $n \geq 1$ temos

$$
\int_{\mathbf{a} \in M^{n}} h(\mathbf{a} x) e^{S_{n}(\mathbf{a} x)}=\beta^{n} h(x) d \mu(\mathbf{a})=0
$$

segue daí que $h(\mathbf{a} x)=0$. Como o conjunto $\left\{\mathbf{a} x, \mathbf{a} \in M^{n}, n=1,2 \ldots: \sigma(\mathbf{a} x)=x\right\}$ é denso em $\Omega$ (aqui precisamos de base enumerável para $M$ ) concluímos que $h \equiv 0$. Por outro lado sabemos que $\beta=\left\|\mathscr{L}_{f} h\right\|_{0}>0$ o que é um absurdo, concluindo a afirmação.

Afirmação 3.: O Autovalor $\beta$ é simples. Para isso suponha que $\mathscr{L}_{f}$ tem uma segunda autofunção $g$ associada ao autovalor $\beta$. Considere

$$
t=\inf _{x \in \Omega}\left\{\frac{g(x)}{h(x)}\right\}=\frac{g(y)}{h(y)}
$$

para algum $y \in \Omega$. Então temos que $g(y)-t h(y)=0$ e pela definição de $t$ que $g(x)-t h(x) \geq 0$ para todo $x \in \Omega$. Aplicando o mesmo procedimento da Afirmação 2 à autofunção $g-t h$ concluímos que $g-t h \equiv 0$ concluindo que $g$ é um múltiplo escalar de $h$.

Sejam $h$ e $\beta$ a autofunção e o autovalor obtidos acima. Defina um novo potencial $g$ pondo $g=f-\log h \circ \sigma-\log h-\log \beta$ é fácil ver que $\mathscr{L}_{g}=\beta^{-1} \Delta(h)^{-1} \circ \mathscr{L}_{f} \circ \Delta(h)$, onde $\Delta(h)$ representa a multiplicação por $h$. Como o espectro de $\mathscr{L}_{g}$ é o espectro de $\mathscr{L}_{f}$ reescalado pelo fator $1 / \beta$ é suficiente provar o teorema supondo que o operador $\mathscr{L}_{f}$ é normalizado. Então, recapitulando, precisamos mostrar que

- A porção do espectro de $\mathscr{L}_{f}: C^{\gamma}(\Omega) \rightarrow C^{\gamma}(\Omega), \sigma\left(\mathscr{L}_{f}\right) \backslash\{1\}$ está contida em um disco com raio estritamente menor que 1 ;

- Existe uma única medida de probabilidade $m$ tal que $\mathscr{L}_{f}^{*} m=m$;

- para cada $\varphi \in C^{\gamma}(\Omega)$ temos a seguinte convergência uniforme $\mathscr{L}_{f}^{n} \varphi \stackrel{\|\cdot\|_{0}}{\longrightarrow} \int \varphi d m$ quando $n \rightarrow \infty$. 
Prova dos itens $(\mathrm{c})$ e $(\mathrm{d})$ :

Afirmação 4. O operador $\mathscr{L}_{f}^{*}:\left(C^{\gamma}(\Omega)\right)^{*} \rightarrow\left(C^{\gamma}(\Omega)\right)^{*}$ preserva o compacto convexo das medidas de probabilidade invariantes pelo shift $\sigma$. Com efeito, seja $m$ uma medida de probabilidade invariante por $\sigma$, e seja $\bar{m}=\mathscr{L}_{f}^{*} m$. Então para cada $\varphi \in C(\Omega)$ temos que

$$
\begin{aligned}
\sigma^{*} \bar{m}(\varphi) & =\int \varphi \circ \sigma d \bar{m} \\
& =\int \varphi \circ \sigma d\left[\mathscr{L}_{f}^{*} m\right] \\
& =\int \mathscr{L}_{f}(\varphi \circ \sigma) d m \\
& =\int \varphi \mathscr{L}_{f}(\mathbf{1}) d m \\
& =\int \varphi d m \\
& =\bar{m}(\varphi) .
\end{aligned}
$$

Portanto segue da continuidade do dual do operador de Ruelle e do Teorema do ponto fixo de Tychonov-Schauder que existe uma medida de probabilidade $m$ tal que $\mathscr{L}_{f}^{*} m=m$.

Para cada $\varphi \in C^{\gamma}(\Omega)$, a desigualdade $\left|\mathscr{L}_{f}^{n} \varphi(x)-\mathscr{L}_{f}^{n} \varphi(y)\right| \leq \operatorname{Hol}\left(\mathscr{L}_{f}^{n} \varphi\right) d(x, y)^{\gamma}$, juntamente com o Lema Principal 2.3.3 nos fornece que $\left\{\mathscr{L}_{f}^{n} \varphi\right\}_{n \in \mathbb{N}}$ é uma sequência uniformemente equicontínua. A hipótese de $f$ ser um potencial normalizado nos fornece fácilmente que $\left\{\mathscr{L}_{f}^{n}\right\}$ é uniformemente limitada. Portanto podemos fazer uso do Teorema de Arzelà-Àscoli e concluir a existência alguma subsequência $\left\{\mathscr{L}_{f}^{n_{k}} \varphi\right\}_{k \in \mathbb{N}}$ convergente na norma da convergência uniforme, para $\varphi^{\star}$ digamos.

Afirmação 5. A função $\varphi^{\star}$ é uma função constante. De fato, primeiro observamos que se $f$ é um potencial normalizado então podemos escrever a seguinte cadeia de desigualdades para qualquer $\varphi \in C^{\gamma}(\Omega)$

$$
\min \varphi \leq \min \mathscr{L}_{f} \varphi \leq \cdots \leq \min \mathscr{L}_{f}^{n} \varphi \leq \cdots \leq \min \varphi^{\star}
$$

Da desigualdade acima temos $\min \varphi^{\star}=\min \mathscr{L}_{f}^{m} \varphi^{\star}$ para todo $m \in \mathbb{N}$. De fato,

$$
\min \left(\mathscr{L}_{f}^{m} \varphi^{\star}\right)=\min \left(\mathscr{L}_{f}^{m}\left(\lim \mathscr{L}_{f}^{n_{k}} \varphi\right)\right)=\min \left(\lim \mathscr{L}_{f}^{n_{k}+m} \varphi\right)=\min \left(\varphi^{\star}\right)
$$

onde a última igualdade segue do fato de que a sequência $\min \left(\mathscr{L}_{f}^{n} \varphi\right)$ é monótona e que $\min \mathscr{L}_{f}^{n_{i}} \varphi \rightarrow \min \varphi^{\star}$.

Agora, dado $\varepsilon>0$ seja $N \in \mathbb{N}$ e $x \in \Omega$ tal que $\min \left(\mathscr{L}_{f}^{k} \varphi^{\star}\right)=\mathscr{L}_{f} \varphi^{\star}(x)$ e $\left\{\mathbf{a} x, \mathbf{a} \in M^{N}\right\}$ é $\varepsilon$-denso em $\Omega$. Pelo exposto acima e pela escolha de $x$ temos que $\mathscr{L}_{f}^{N} \varphi^{\star}(x)=\min \left(\varphi^{\star}\right)$. Seja $z \in \Omega$ tal que $\min \left(\varphi^{\star}\right)=\varphi^{\star}(z)$, então

$$
\int_{\mathbf{a} \in M^{N}} e^{S_{n} f(\mathbf{a} x)} \varphi^{\star}(\mathbf{a} x) d \mu(\mathbf{a})=\mathscr{L}^{N} \varphi^{\star}(x)=\min \left(\varphi^{\star}\right)=\varphi^{\star}(z) .
$$


Usando a identidade $\mathbf{1}(x)=\mathscr{L}_{\log g}^{N} \mathbf{1}(x)$, segue da equação anterior que

$$
0=\int_{\mathbf{a} \in M^{N}} e^{S_{n} f(\mathbf{a} x)}\left[\varphi^{\star}(\mathbf{a} x)-\varphi^{\star}(z)\right] d \mu(\mathbf{a}) .
$$

Usando a continuidade de $\varphi^{\star}$ e o fato de $\operatorname{supp}(\mu)=M$ é fácil ver que $\varphi^{\star}(\mathbf{a} x)=\varphi^{\star}(z)$ para qualquer $\mathbf{a} \in M^{N}$. Visto que $\varphi^{\star}$ contínua e constante em $\left\{\mathbf{a} x, \mathbf{a} \in M^{N}\right\}$, o qual é $\varepsilon$-denso em $\Omega$, segue que $\varphi^{\star}$ é constante.

Pela definição da medida $m$ temos que a seguinte igualdade é válida $m=\left(\mathscr{L}_{f}^{*}\right)^{n_{k}}(m)$ para todo $n_{k} \in \mathbb{N}$. Assim, por um lado

$$
\lim _{k \rightarrow \infty} \int\left(\mathscr{L}_{f}^{n_{k}} \varphi\right) d m=\lim _{k \rightarrow \infty}\left(\mathscr{L}_{f}^{*}\right)^{n_{k}}(m)(\varphi)=m(\varphi)=\int \varphi d m
$$

por outro lado

$$
\lim _{k \rightarrow \infty} \int\left(\mathscr{L}_{f}^{n_{k}} \varphi\right) d m=\int \lim _{k \rightarrow \infty}\left(\mathscr{L}_{f}^{n_{k}} \varphi\right) d m=\int \varphi^{\star} d m=\varphi^{\star}=\lim _{k \rightarrow \infty}\left(\mathscr{L}_{f}^{n_{k}} \varphi\right)
$$

concluímos que

$$
\lim _{k \rightarrow \infty}\left(\mathscr{L}_{f}^{n_{k}} \varphi\right)=\int \varphi d m
$$

Como $C^{\gamma}(\Omega)$ é uniformemente denso em $C(\Omega)$ a menos de um argumento do tipo " $\varepsilon / 3$ " podemos assumir que a igualdade acima é verdadeira para todo $\varphi \in C(\Omega)$.

Agora vamos mostrar que $\mathscr{L}_{f}^{n} \varphi$ é convergente. Primeiro observamos que todo o argumento usado para provar que $\varphi^{\star}$ é constante é independente da escolha da subsequência. Agora considere uma outra subsequência $\left\{\mathscr{L}_{f}^{l_{k}} \varphi\right\}_{k \in \mathbb{N}}$ convergindo uniformemente para $\varphi_{1}^{\star}$, digamos. Pelo que acabamos de mencionar segue que $\varphi_{1}^{\star}$ é uma função constante. Sendo assim temos que

$$
\lim _{k \rightarrow \infty} \int\left(\mathscr{L}_{f}^{l_{k}} \varphi\right) d m=\lim _{k \rightarrow \infty}\left(\mathscr{L}_{f}^{*}\right)^{l_{k}}(m)(\varphi)=m(\varphi)=\int \varphi d m .
$$

Por outro lado,

$$
\lim _{k \rightarrow \infty} \int\left(\mathscr{L}_{f}^{l_{k}} \varphi\right) d m=\int \lim _{k \rightarrow \infty}\left(\mathscr{L}_{f}^{l_{k}} \varphi\right) d m=\int \varphi_{1}^{\star} d m=\varphi_{1}^{\star}=\lim _{k \rightarrow \infty}\left(\mathscr{L}_{f}^{l_{k}} \varphi\right)
$$

$\log 0$

$$
\lim _{k \rightarrow \infty}\left(\mathscr{L}_{f}^{l_{k}} \varphi\right)=\int \varphi d m .
$$

Em particular, concluímos que toda subsequência de $\left\{\mathscr{L}^{n} \varphi\right\}$ tem uma subsequência convergente as quais convergem para o mesmo valor $\int \varphi d m$, segue daí o desejado,

$$
\lim _{n \rightarrow \infty} \mathscr{L}_{f}^{n} \varphi=\int \varphi d m
$$

Este fato conclui as provas dos itens $(c)$ e $(d)$.

Prova do item (b). Para provar o item (b) precisaremos do seguinte lema, que também será útil no Capítulo 6, Lema 6.1.3. 
Lema 2.3.5. A projeção $\Pi: C^{\gamma}(\Omega) \rightarrow$ Eig $\left(\lambda_{f}\right)$ que projeta $\varphi$ sobre o autoespaço associado a $\lambda_{f}$ é dada por $\Pi(\varphi)=\left(\int \varphi d m\right) \cdot h$.

Deste lema temos imediatamente que $\Pi(\varphi)=0$ se, e somente se, $\int \varphi d m=0$. Já que toda função $\varphi \in C^{\gamma}(\Omega)$ pode ser escrita como $\varphi=\left(\int \varphi d m\right) h+\left[\varphi-\left(\int \varphi d m\right) h\right]$ e que $\int h d m=1$, segue que o espaço $C^{\gamma}(\Omega)$ pode ser escrito como a soma direta $C^{\gamma}(\Omega)=\operatorname{Eig}\left(\lambda_{f}\right) \oplus \mathcal{K}$, onde

$$
\mathcal{K}=\left\{\varphi \in C^{\gamma}(\Omega) ; \int \varphi d m=0\right\} .
$$

Note que para todo $\varphi \in \mathcal{K}$ temos da definição da medida $m$ que

$$
\int \mathscr{L}_{f} \varphi d m=\int \varphi d\left[\mathscr{L}_{f} m\right]=\int \varphi d m=0 .
$$

Deste fato segue que $\mathscr{L}_{f}(\mathcal{K}) \subset \mathcal{K}$. Já que $h$ é uma auto-função de $\mathscr{L}_{f}$ a decomposição em soma direta acima induz a seguinte decomposição de $\mathscr{L}_{f}=\left.\left.\mathscr{L}_{f}\right|_{\operatorname{Eig}\left(\lambda_{f}\right)} \oplus \mathscr{L}_{f}\right|_{\mathcal{K}}$.

Assim para provar o item (b) basta mostrar que $\mathscr{L}_{f} \mid \mathcal{K}: \mathcal{K} \rightarrow \mathcal{K}$ tem raio espectral estritamente menor que 1. Para facilitar a notação vamos chamar de $\mathcal{B}$ a bola unitária fechada do espaço $\mathcal{K}$, isto é,

$$
\mathcal{B}=\left\{\varphi \in C^{\gamma}(\Omega) ; \int \varphi d m=0 ;\|\varphi\|_{\gamma} \leq 1\right\}
$$

Afirmação 6. Dado $\varepsilon>0$ existe $N=N_{\varepsilon}$ tal que $\left\|\mathscr{L}_{f}^{n} \varphi\right\|_{0}<\varepsilon, \forall n \geq N, \forall \varphi \in \mathcal{B}$. De fato, considere a família $B_{n}:=\left\{\varphi \in \mathcal{K} ;\left\|\mathscr{L}^{m} \varphi\right\|_{0}<\varepsilon, \forall m \geq n\right\}$. Como $f$ é um potencial normalizado temos que $\left\|\mathscr{L}_{f}^{n+1} \varphi\right\|_{0} \leq\left\|\mathscr{L}_{f}^{n} \varphi\right\|_{0}$ e portanto $B_{n} \subset B_{n+1}$. Além disso temos que cada $B_{n}$ é aberto de $C(\Omega)$. Para todo $\varphi \in \mathcal{K}$ temos do item (d) que $\mathscr{L}_{f}^{n} \varphi \rightarrow 0$. Logo $\mathcal{B} \subset \bigcup_{n \in \mathbb{N}} B_{n}$. Como $\mathcal{B}$ é um compacto de $C(\Omega)$ a cobertura aberta de $\mathcal{B}$, pelos abertos $B_{n}$ 's, admite uma subcobertura finita. Uma vez que esta coleção é encaixada podemos então encontrar algum $N \in \mathbb{N}$ tal que $\mathcal{B} \subset B_{N}$ e assim a afirmação está provada.

Pelo Lema 3.1.1 temos que

$$
\begin{aligned}
\operatorname{Hol}\left(\mathscr{L}_{f}^{n+k} \varphi\right) & \leq C \cdot\left\|\mathscr{L}_{f}^{k} \varphi\right\|_{0}+\frac{1}{2^{n \gamma}} \operatorname{Hol}\left(\mathscr{L}_{f}^{k} \varphi\right) \\
& =C \cdot\left\|\mathscr{L}_{f}^{k} \varphi\right\|_{0}+\frac{1}{2^{n \gamma}}\|\varphi\|_{0}+\frac{1}{2^{(n+k) \gamma}} \operatorname{Hol}(\varphi) .
\end{aligned}
$$

A desigualdade acima juntamente com a Afirmação 6 deixam claro que para $n$ e $k$ suficientemente grande temos $\left\|\mathscr{L}_{f}^{n+k} \varphi\right\|_{\gamma} \leq \varepsilon<1, \quad \forall \varphi \in \mathcal{K},\|\varphi\|_{\gamma} \leq 1$. Lembramos que se $\lambda \in \sigma\left(\left.\mathscr{L}_{f}\right|_{\mathcal{K}}\right)$ então $\lambda^{n+k} \in \sigma\left(\left.\mathscr{L}_{f}^{n+k}\right|_{\mathcal{K}}\right)$ e também que $\sigma\left(\left.\mathscr{L}_{f}^{n+k}\right|_{\mathcal{K}}\right) \subset D\left(0,\left\|\left(\left.\mathscr{L}_{f}^{n+k}\right|_{\mathcal{K}}\right)\right\|_{\gamma}\right)$. Podemos concluir que $|\lambda|^{n+k} \leq\left\|\left(\left.\mathscr{L}_{f}^{n+k}\right|_{\mathcal{K}}\right)\right\|_{\gamma}$ para todo $\lambda \in \sigma\left(\left.\mathscr{L}_{f}\right|_{\mathcal{K}}\right)$. Juntando estas duas últimas desigualdades temos

$$
|\lambda|^{n+k} \leq\left\|\left(\left.\mathscr{L}_{f}^{n+k}\right|_{\mathcal{K}}\right)\right\|_{\gamma}<\varepsilon \Rightarrow|\lambda|<\varepsilon^{\frac{1}{n+k}} \quad \forall \lambda \in \sigma\left(\left.\mathscr{L}_{f}\right|_{\mathcal{K}}\right) .
$$

Da última desigualdade decorre que o raio espectral de $\left.\mathscr{L}_{f}\right|_{\mathcal{K}}$ é menor ou igual que $\varepsilon^{\frac{1}{n+k}}$ que é estritamente menor que um. Com isto finalizamos a prova do item (b) e também a prova do Teorema de Ruelle-Perron-Fröbenius. 


\section{Algumas observações a Respeito Do TeORema 2.3 .4}

1. Seja $f$ um potencial não normalizado e denote por $\bar{f}=f+\log h_{f}-\log h_{f} \circ \sigma-\log \lambda_{f}$ o potencial normalizado associado. Seja $m$ a medida de probabilidade que satisfaz $\mathscr{L}_{\bar{f}}^{*} m=m$ dado pelo teorema anterior. Então a medida $\nu_{f}=\left(1 / h_{f}\right) \cdot m$ satisfaz $\mathscr{L}_{f}^{*} \nu_{f}=\lambda_{f} \nu_{f}$. De fato, para toda $\varphi \in C(\Omega)$ temos

$$
\begin{aligned}
\int \varphi d m & =\int \varphi d\left[\mathscr{L}_{\bar{f}}^{*} m\right]=\int \mathscr{L}_{\bar{f}} \varphi d m \\
& =\int\left(\int \varphi(a x) e^{\bar{f}(a x)} d \mu(a)\right) d m(x) \\
& =\int\left(\int \varphi(a x) \frac{e^{f(a x)} h_{f}(a x)}{\lambda_{f} h_{f}(x)} d \mu(a)\right) d m(x) .
\end{aligned}
$$

Substituindo, na expressão acima, $\varphi$ por $\varphi / h_{f}$ ficamos com

$$
\int \frac{\varphi}{h_{f}} d m=\frac{1}{\lambda_{f}} \int\left(\int \varphi(a x) e^{f(a x)} d \mu(a)\right) \frac{1}{h_{f}(x)} d m(x)
$$

o que é equivalente a dizer que

$$
\lambda_{f} \int \varphi d \nu_{f}=\int\left(\mathscr{L}_{f} \varphi\right) d \nu_{f}
$$

ou seja, $\mathscr{L}_{f}^{*} \nu_{f}=\lambda_{f} \nu_{f}$.

2. Para qualquer potencial $\varphi \in C(\Omega)$ temos que $\lambda_{f}^{-n} \mathscr{L}_{f} \varphi \rightarrow h_{f} \int \varphi d \nu_{f}$, na norma da convergência uniforme em $C(\Omega)$. Para provar este fato, primeiro observamos que $f=\bar{f}-\log h_{f}+$ $\log h_{f} \circ \sigma+\log \lambda f$. Segue daí, por um argumento de somas telescópicas, que

$$
S_{n} f=S_{n} \bar{f}-\log h_{f}+\log h_{f} \circ \sigma^{n}+n \log \lambda_{f} .
$$

O que nos fornece

$$
\begin{aligned}
\lambda_{f}^{-n} \mathscr{L}_{f}^{n} \varphi(x) & =\lambda_{f}^{-n} \int \varphi(\mathbf{a} x) e^{S_{n} f(\mathbf{a} \underline{x})} d \mu(\mathbf{a}) \\
& =h_{f}(\underline{x}) \int \varphi(\mathbf{a} x) \frac{e^{S_{n} \bar{f}(\mathbf{a} x)}}{h_{f}(\mathbf{a} x)} d \mu(\mathbf{a}) \\
& =h_{f}(x) \mathscr{L}_{\bar{f}}^{n}\left(\frac{\varphi}{h_{f}}\right) .
\end{aligned}
$$

Por outro lado, segue do teorema anterior que

$$
\mathscr{L}_{\bar{f}}^{n}\left(\frac{\varphi}{h_{f}}\right) \rightarrow \int \frac{\varphi}{h_{f}} d m=\int \varphi d \nu_{f}
$$

uniformemente quando $n \rightarrow \infty$. 
Proposição 2.3.6. Se $m$ é o ponto fixo para o operador de Ruelle obtido pelo teorema anterior, então o sistema $(\sigma, m)$ é mixing.

Demonstração. Sejam $\varphi, \psi \in C(\Omega)$, como $m=\left(\mathscr{L}_{f}^{*}\right)^{n} m$, para todo $n \in \mathbb{N}$, temos que

$$
\begin{aligned}
\int \varphi \cdot\left(\psi \circ \sigma^{n}\right) d m & =\int \varphi \cdot\left(\psi \circ \sigma^{n}\right) d\left[\left(\mathscr{L}_{f}^{*}\right)^{n} m\right] \\
& =\int \mathscr{L}_{f}^{n}\left(\varphi \cdot\left(\psi \circ \sigma^{n}\right)\right) d[m] \\
& =\int \psi \cdot \mathscr{L}_{f}^{n} \varphi d m
\end{aligned}
$$

e como $\mathscr{L}_{f}^{n} \varphi \rightarrow \int \varphi d m$ temos que $\int \varphi \cdot\left(\psi \circ \sigma^{n}\right) d m \rightarrow \int \varphi d m \int \psi d m$, donde segue o resultado. 


\section{Capítulo 3}

\section{Analiticidade do Funcional Pressão E A PRESEnÇA Do Buraco EspeCtral}

\subsection{Analiticidade do Operador de Ruelle em RelaÇão AO POTENCIAL}

Uma importante propriedade do operador de Ruelle é sua dependência analítica ( no sentido de Frechet) com respeito ao potencial. De modo mais preciso, temos o lema abaixo.

Lema 3.1.1. O mapa $\Theta: C^{\gamma}(\Omega) \rightarrow \mathcal{L}\left(C^{\gamma}(\Omega), C^{\gamma}(\Omega)\right)$ que envia $f \in C^{\gamma}(\Omega)$ no Operador de Ruelle $\mathscr{L}_{f}$ associado ao potencial $f$, é analítico.

Demonstração. Veja [4] Teorema 3.5.

O principal objetivo desta seção é estender o resultado enunciado acima para os espaços de Walters $W(\Omega)$, uma classe de espaços que vão ser definidos futuramente e que vão conter o espaço $C^{\gamma}(\Omega)$ e vão desempenhar papel importante nos próximos capítulos. Vamos provar que o operador de Ruelle e seu dual dependem analiticamente com respeito ao potencial quando estes estão em $C^{\gamma}(\Omega)$ e então derivar a analiticidade da pressão. No intuito de formular esses resultados em um contexto unificado iremos introduzir alguma notação adicional.

Seja $\mathcal{K} \subset C(\Omega)$ um subespaço vetorial arbitrário de $C(\Omega)$, equipado com uma norma $\|\cdot\|$. Usaremos a notação $\mathcal{K}^{*}$ para denotar o dual topológico de $(\mathcal{K},\|\cdot\|)$. Definimos a norma de um elemento $\phi \in \mathcal{K}^{*}$ usualmente pondo $\|\phi\|_{*}=\sup \{|\phi(f)|: f \in \mathcal{K}$ and $\|f\|=1\}$. Para não deixar a notação muito carregada, o espaço $\mathcal{L}(\mathcal{K}, \mathcal{K})$ de todos os operadores lineares contínuos (na topologia forte) agindo em $\mathcal{K}$ será denotado por $V \equiv \mathcal{L}(\mathcal{K}, \mathcal{K})$.

Definição 3.1.2. Seja $\mathcal{K} \subset C(\Omega)$ um subespaço vetorial. Diremos que $\mathcal{K}$ é invariante para o operador de Ruelle, quando para todo $f \in \mathcal{K}$ tenhamos que $\mathscr{L}_{f} \mathcal{K} \subset \mathcal{K}$.

Exemplos centrais de subespaços invariantes pelo operador de Ruelle são os espaços $C^{\gamma}(\Omega)$, $0<\gamma \leq 1$ e o espaço de Walters $W(\Omega)$. A próxima proposição desempenha um papel funda- 
mental no estudo da analiticidade da pressão. Ela constitui uma generalização significativa do Teorema 3.5 in [44].

Proposição 3.1.3. Seja $\mathcal{K} \subset C(\Omega)$ um subespaço equipado com uma norma $\|\cdot\|$ de tal maneira que $(\mathcal{K},\|\cdot\|)$ seja uma álgebra de Banach. Suponha que $\mathcal{K}$ seja invariante para o operador de Ruelle e que para qualquer $f \in \mathcal{K}$ tenhamos $\mathscr{L}_{f} \in V$. Então o mapas $\Theta$ e $\Theta^{*}$ dados por

$$
\mathcal{K} \ni f \mapsto \mathscr{L}_{f} \in \mathcal{L}(\mathcal{K}, \mathcal{K}) \quad e \quad \mathcal{K} \ni f \mapsto \mathscr{L}_{f}^{*} \in \mathcal{L}\left(\mathcal{K}^{*}, \mathcal{K}^{*}\right)
$$

são analíticos.

Antes de provar a proposição acima, vamos enunciar um seu corolário imediato que é uma ferramenta importante para a obter analiticidade do funcional pressão.

Corolário 3.1.4. Para cada $0<\gamma \leq 1$, ambos os mapas

$$
C^{\gamma}(\Omega) \ni f \mapsto \mathscr{L}_{f} \in \mathcal{L}\left(C^{\gamma}(\Omega), C^{\gamma}(\Omega)\right) \quad e \quad C^{\gamma}(\Omega) \ni f \mapsto \mathscr{L}_{f}^{*} \in \mathcal{L}\left(C^{\gamma}(\Omega)^{*}, C^{\gamma}(\Omega)^{*}\right)
$$

definem mapas analíticos.

Demonstração. Basta observar que o subespaço $C^{\gamma}(\Omega)$ é invariante para o operador de Ruelle e o espaço $\left(C^{\gamma}(\Omega),\|\cdot\|_{\gamma}\right)$ é uma álgebra de de Banach (ver [44]).

\section{Prova da Proposição 3.1.3.}

Provaremos primeiro a analiticidade de $\Theta$. Dados $f, h \in \mathcal{K}$ e $\varphi \in C(\Omega)$ temos para qualquer $x \in \Omega$ que

$$
\begin{aligned}
\Theta(f+h)(\varphi)(x)-\Theta(f)(\varphi)(x) & =\mathscr{L}_{f+h}(\varphi)(x)-\mathscr{L}_{f}(\varphi)(x) \\
& =\int_{M} e^{f(a x)+h(a x)} \varphi(a x) d \mu(a)-\int_{M} e^{f(a x)} \varphi(a x) d \mu(a) \\
& =\int_{M} e^{f(a x)} \varphi(a x)\left(e^{h(a x)}-1\right) d \mu(a) \\
& =\int_{M}\left(e^{f(a x)} \varphi(a x) \sum_{n=1}^{\infty} \frac{[h(a x)]^{n}}{n !}\right) d \mu(a) .
\end{aligned}
$$

Assumindo que podemos aplicar o teorema de Fubini obtemos

$$
\Theta(f+h)(\varphi)(x)-\Theta(f)(h)(x)=\sum_{n=1}^{\infty} \frac{1}{n !} \int_{M} e^{f(a x)} \varphi(a x)[h(a x)]^{n} d \mu(a),
$$

que reescrita em termos de $\Theta$ fica, $\Theta(f+h)(\varphi)(x)-\Theta(f)(\varphi)(x)=\sum_{n=1}^{\infty}(1 / n !) \Theta(f)\left(\varphi \cdot h^{n}\right)(x)$. Esta igualdade pode ser reescrita, omitindo-se a dependência $x$ e $\varphi$, tomando o seguinte aspecto

$$
\Theta(f+h)-\Theta(f)=\sum_{n=1}^{\infty} \frac{1}{n !} \Theta(f)\left((\cdot) h^{n}\right) .
$$


Com o objetivo de justificar a aplicação do Teorema de Fubini acima é suficiente provar que a soma acima converge em $(\mathcal{K},\|\cdot\|)$. Repare primeiro que para quaisquer $h_{1}, \ldots, h_{k}$ in $\mathcal{K}$, o mapa $\varphi \mapsto \Theta(f)\left(\varphi h_{1} \ldots h_{k}\right)$ de $\mathcal{K}$ em si mesmo define um operador linear contínuo, i.e., $\Theta(f)\left((\cdot) h_{1} \ldots h_{k}\right) \in V$ cuja norma é limitada por

$$
\left\|\Theta(f)\left((\cdot) h_{1} \ldots h_{k}\right)\right\|_{V} \leq\left\|\mathscr{L}_{f}\right\|_{V}\left\|h_{1}\right\| \ldots\left\|h_{k}\right\|,
$$

de fato, basta observar que $\mathscr{L}_{f} \in V$ e que $(\mathcal{K},\|\cdot\|)$ é uma álgebra de Banach. Segue desta desigualdade que

$$
\sum_{n=1}^{\infty} \frac{1}{n !}\left\|\Theta(f)\left((\cdot)[h]^{n}\right)\right\|_{V} \leq \sum_{n=1}^{\infty} \frac{1}{n !}\left\|\mathscr{L}_{f}\right\|_{V}(\|h\|)^{n}=\left\|\mathscr{L}_{f}\right\|_{V}\left\{e^{\|h\|}-1\right\}
$$

que implica a convergência da série $\sum_{n=1}^{\infty}(1 / n !) \Theta(f)\left((\cdot)[h]^{n}\right)$ em $V$.

Afirmação 1. Para qualquer $k \in \mathbb{N}$ e $h_{1}, \ldots, h_{k} \in \mathcal{K}$, temos que

$$
D^{k} \Theta(f)\left(h_{1}, \ldots, h_{k}\right)=\Theta(f)\left((\cdot) h_{1} \cdots h_{k}\right) .
$$

A verificação será executada através de indução em $k$. No que segue $\mathcal{L}^{k}=\mathcal{L}^{k}(\mathcal{K}, V)$ denota o espaço das aplicações $k$-lineares $l: \mathcal{K} \times \ldots \times \mathcal{K} \rightarrow V$, de $\mathcal{K} \times \ldots \times \mathcal{K}(k-$ cópias de $\mathcal{K})$ em $V$. A norma $\|\cdot\|_{\mathcal{L}^{k}}$ de $\mathcal{L}^{k}$ é dada por

$$
\|l\|_{\mathcal{L}^{k}}=\sup _{\substack{\left\|h_{i}\right\|=1 \\ i=1, \ldots, k}}\left\|l\left(h_{1}, \ldots, h_{k}\right)\right\|_{V}, \quad l \in \mathcal{L}^{k}
$$

Vamos provar inicialmente a afimação para $k=1$. Neste caso temos de (3.1) que

$$
\Theta\left(f+h_{1}\right)-\Theta(f)=\Theta(f)\left((\cdot) h_{1}\right)+\mathcal{O}_{1}\left(h_{1}\right)
$$

onde, $\mathcal{O}_{1}\left(h_{1}\right)=\sum_{n=2}^{\infty}(1 / n !) \Theta(f)\left((\cdot)\left[h_{1}\right]^{n}\right)$. A desigualdade 3.2 implica que $\left\|\Theta(f)\left((\cdot) h_{1}\right)\right\|_{V} \leq$ $\left\|\mathscr{L}_{f}\right\|_{V}\left\|h_{1}\right\|$ e portanto $h_{1} \mapsto \Theta(f)\left((\cdot) h_{1}\right)$ está em $\mathcal{L}^{1}$. Novamente, em vista da desigualdade (3.2) temos

$$
\left\|\mathcal{O}_{1}\left(h_{1}\right)\right\|_{V}=\left\|\sum_{n=2}^{\infty} \frac{1}{n !} \Theta(f)\left((\cdot)\left[h_{1}\right]^{n}\right)\right\|_{V} \leq \sum_{n=2}^{\infty} \frac{1}{n !}\left\|\mathscr{L}_{f}\right\|_{V}(\|h\|)^{n}
$$

mostrando que $\left(1 /\left\|h_{1}\right\|\right)\left\|\mathcal{O}_{1}\left(h_{1}\right)\right\|_{V} \rightarrow 0$, quando $\left\|h_{1}\right\| \rightarrow 0$. Portanto, $D^{1} \Theta(f)\left(h_{1}\right)=\Theta(f)\left((\cdot) h_{1}\right)$ e a afirmação é verdade para $k=1$. Agora, vamos supor que a afirmação seja verdade para $k-1, k \geq 2$, isto é,

$$
D^{k-1} \Theta(f)\left(h_{1}, \ldots, h_{k-1}\right)=\Theta(f)\left((\cdot) h_{1} \ldots h_{k-1}\right), h_{1}, \ldots, h_{k-1} \in \mathcal{K} .
$$

Vamos verificar que a afirmação é verdade para $k$, isto é,

$$
D^{k} \Theta(f)\left(h_{1}, \ldots, h_{k}\right)=\Theta(f)\left((\cdot) h_{1} \cdots h_{k}\right), h_{1}, \ldots, h_{k} \in \mathcal{K} .
$$

Pela hipótese de indução (3.3), dados $h_{1}, \ldots h_{k-1}, h_{k}$ e $h$ em $\mathcal{K}$ temos,

$$
\begin{aligned}
D^{k-1} \Theta\left(f+h_{k}\right)\left(h_{1}, \ldots, h_{k-1}\right)(h)-D^{k-1} \Theta(f) & \left(h_{1}, \ldots, h_{k-1}\right)(h) \\
& =\Theta\left(f+h_{k}\right)\left(h h_{1} \ldots h_{k-1}\right)-\Theta(f)\left(h h_{1} \ldots h_{k-1}\right) .
\end{aligned}
$$


De (3.1) segue que

$$
\begin{aligned}
D^{k-1} \Theta\left(f+h_{k}\right)\left(h_{1}, \ldots, h_{k-1}\right)(h)-D^{k-1} \Theta(f)\left(h_{1}, \ldots, h_{k-1}\right) & (h) \\
= & =\sum_{n=1}^{\infty} \frac{1}{n !} \Theta(f)\left(h h_{1} \ldots h_{k-1}\left[h_{k}\right]^{n}\right) .
\end{aligned}
$$

Claramente, a equação anterior mostra que

$$
\begin{aligned}
D^{k-1} \Theta\left(f+h_{k}\right)\left(h_{1}, \ldots, h_{k-1}\right)-D^{k-1} \Theta(f)\left(h_{1}, \ldots, h_{k-1}\right) & \\
& \left.=\Theta(f)(\cdot) h_{1} \ldots h_{k-1} h_{k}\right)+\mathcal{O}_{k}\left(h_{k}\right)\left(h_{1} \ldots h_{k-1}\right),
\end{aligned}
$$

onde $\mathcal{O}_{k}\left(h_{k}\right)$ é um elemento de $L^{k-1}$ dado por

$$
\mathcal{O}_{k}\left(h_{k}\right)\left(h_{1} \ldots h_{k-1}\right)=\sum_{n=2}^{\infty} \frac{1}{n !} \Theta(f)\left((\cdot) h_{1} \ldots h_{k-1}\left[h_{k}\right]^{n}\right) .
$$

Estas cotas superiores juntamente com a desigualdade 3.2 nos permitem concluir que o mapa $\left(h_{1} \ldots h_{k}\right) \mapsto \Theta(f)\left((\cdot) h_{1} \ldots h_{k}\right)$ é um elemento de $\mathcal{L}^{k}$. A desigualdade $(3.2)$ e a definição de $\mathcal{O}_{k}\left(h_{k}\right)$ nos dá a cota superior

$$
\left\|\mathcal{O}_{k}\left(h_{k}\right)\left(h_{1} \ldots h_{k-1}\right)\right\|_{V} \leq \sum_{n=2}^{\infty} \frac{1}{n !}\left\|\mathscr{L}_{f}\right\|_{V}\left\|h_{1}\right\| \ldots\left\|h_{k-1}\right\|\left(\left\|h_{k}\right\|\right)^{n}
$$

donde $\left(1 /\left\|h_{k}\right\|\right)\left\|\mathcal{O}_{k}\left(h_{k}\right)\right\|_{V} \rightarrow 0$, quando $\left\|h_{k}\right\| \rightarrow 0$. Portanto, $D^{k} \Theta(f)\left(h_{1}, \ldots h_{k}\right)=\Theta(f)\left((\cdot) h_{1} \ldots h_{k}\right)$ e a afirmação está provada. Usando a Afirmação 1 e as estimativas acima para estimar o resto, segue a analiticidade de $\mathcal{K} \ni f \mapsto \mathscr{L}_{f} \in V$.

Analiticidade de $\Theta^{*}$. Sejam $f, g$ e $h$ em $\mathcal{K}$ e $\phi^{*} \in \mathcal{K}^{*}$. Pela expansão dada em 3.1 para $\Theta(f+h)$ temos

$$
\begin{aligned}
\Theta^{*}(f+h)\left(\phi^{*}\right) g=\phi^{*}(\Theta(f+h)(g)) & =\phi^{*}\left(\sum_{n=0}^{\infty} \frac{1}{n !} \Theta(f)\left(g[h]^{n}\right)(\cdot)\right) \\
& =\sum_{n=0}^{\infty} \frac{1}{n !} \phi^{*}\left(\Theta(f)\left(g[h]^{n}\right)(\cdot)\right) \\
& =\sum_{n=0}^{\infty} \frac{1}{n !}\left(\Theta^{*}(f) \phi^{*}\right)\left(g[h]^{n}\right)(\cdot) .
\end{aligned}
$$

Afirmação 2. Considere a derivada $D \Theta^{*}: \mathcal{K} \rightarrow L\left(\mathcal{K}, L\left(\mathcal{K}^{*}, \mathcal{K}^{*}\right)\right)$, então, para qualquer $f \in \mathcal{K}$ e $h \in \mathcal{K}$ temos que $D \Theta^{*}(f)(h): \mathcal{K}^{*} \rightarrow \mathcal{K}^{*}$ é dada por $\left(D \Theta^{*}(f)(h)\left(\phi^{*}\right)\right) g=\left(\Theta^{*}(f)\left(\phi^{*}\right)\right)(g h)$. De fato, considere $\mathcal{O}: \mathcal{K} \rightarrow L\left(\mathcal{K}^{*}, \mathcal{K}^{*}\right)$ definida por $\mathcal{O}(h)\left(\phi^{*}\right)=\sum_{n=2}^{\infty}(1 / n !) \Theta^{*}\left(\phi^{*}\right)\left((\cdot)[h]^{n}\right)$ então temos

$$
\begin{aligned}
\|\mathcal{O}(h)\|_{L\left(\mathcal{K}^{*}, \mathcal{K}^{*}\right)}=\sup _{\left\|\phi^{*}\right\|_{*}=1}\left\|\mathcal{O}(h)\left(\phi^{*}\right)\right\|_{*}=\sup _{\left\|\phi^{*}\right\|_{*}=1} & \left\|\sum_{n=2}^{\infty} \frac{1}{n !} \Theta^{*}(f)\left(\phi^{*}\right)\left((\cdot)[h]^{n}\right)\right\|_{*} \\
& \leq \sup _{\left\|\phi^{*}\right\|_{*}=1} \sum_{n=2}^{\infty} \frac{1}{n !} \underbrace{\left\|\Theta^{*}(f)\left(\phi^{*}\right)\left((\cdot)[h]^{n}\right)\right\|_{*}}_{I} .
\end{aligned}
$$


O próximo passo é limitar $I$.

$$
\begin{aligned}
I=\left\|\Theta^{*}(f)\left(\phi^{*}\right)\left((\cdot)[h]^{n}\right)\right\|_{*} & =\sup _{\|g\|=1}\left\|\Theta^{*}(f)\left(\phi^{*}\right)\left((g)[h]^{n}\right)\right\|=\sup _{\|g\|=1}\left\|\phi^{*}\left(\Theta(f)\left((g)[h]^{n}\right)\right)\right\| \\
& \leq\left\|\phi^{*}\right\|_{*} \sup _{\|g\|=1}\left\|\Theta(f)\left((g)[h]^{n}\right)\right\| \leq\left\|\phi^{*}\right\|_{*}\|\Theta(f)\|_{V} \sup _{\|g\|=1}\left\|g[h]^{n}\right\| \\
& \leq\left\|\phi^{*}\right\|_{*}\|\Theta(f)\|_{V} \sup _{\|g\|=1}\|g\|\left\|[h]^{n}\right\| \leq C\|h\|^{n} .
\end{aligned}
$$

Substituindo 3.7 em 3.6 temos que

$$
\|\mathcal{O}(h)\|_{L\left(\mathcal{K}^{*}, \mathcal{K}^{*}\right)} \leq \sum_{n=2}^{\infty} \frac{1}{n !} C\|h\|
$$

portanto $\|\mathcal{O}(h)\|_{L\left(\mathcal{K}^{*}, \mathcal{K}^{*}\right)} /\|h\| \rightarrow 0$, quando $\|h\| \rightarrow 0$.

Podemos mostrar que as derivadas de ordem mais altas $D^{k} \Theta^{*}: \mathcal{K} \rightarrow L\left(\mathcal{K}^{k}, \mathcal{L}\left(\mathcal{K}^{*}, \mathcal{K}^{*}\right)\right)$ para $k \geq 2$, são dadas por

$$
D^{k} \Theta^{*}(f)\left(h_{1}, \ldots, h_{k}\right) \phi^{*}(g)=\Theta^{*}(f) \phi^{*}\left(h_{1} \cdots h_{k} g\right)=\phi^{*}\left(\mathscr{L}_{f}\left(h_{1} \cdots h_{k} g\right)\right) .
$$

A prova é similar a anterior e será omitida.

\subsection{O Buraco Espectral e a Analiticidade da Pressão}

Entendemos por presença de buraco espectral a existência de um autovalor isolado de módulo máximo. A presença do buraco espectral no operador de Ruelle é uma propriedade fundamental para provar a analiticidade da pressão, além disso o buraco espectral implica no decaimento exponencial de correlações com respeito às medidas de Gibbs. Estes resultados são bastante conhecidos no contexto potenciais Hölder quando o espaço de estados $M$ é finito. Nesta secção vamos analisar generalizações deste resultado no contexto em que $M$ é um espaço métrico compacto separável e potenciais mais gerais.

A principal diferença entre o Teorema de Ruelle para $f \in W(\Omega)$ e para $f \in C^{\gamma}(\Omega)$ é o fato de que no primeiro caso não temos muita informação a respeito do espectro de $\mathscr{L}_{f}$. De fato, não é um problema fácil decidir quando temos a presença de buraco espectral para o operador de Ruelle $\mathscr{L}_{f}$, o que vem a ser algo crucial no entendimento da medida de Gibbs associada.

Antes de prosseguir, vamos introduzir uma definição importante em toda esta seção.

Definição 3.2.1. Seja $f \in C(\Omega)$ e considere $\mathscr{L}_{f}$ o operador de Ruelle associado. Diremos que o Teorema de Ruelle vale para $\mathscr{L}_{f}$ se este operador goza das seguintes propriedades:

(a) Existe uma autofunção $h_{f}$ positiva e uma automedida Boreliana $\nu_{f}$ e um número real positivo $\lambda_{f}$ tais que $\mathscr{L}_{f} h_{f}=\lambda_{f} h_{f}$ e $\mathscr{L}_{f}^{*} \nu_{f}=\lambda_{f} \nu_{f}$; 
(b) Para toda função contínua $\varphi \in C(\Omega)$ tenhamos que

$$
\left\|\lambda_{f}^{-n} \mathscr{L}_{f}^{n} \varphi-h_{f} \int_{\Omega} \varphi d \nu_{f}\right\|_{0} \rightarrow 0
$$

quando $n \rightarrow \infty$.

Observe que quando $f \in C^{\gamma}(\Omega)$ o Teorema de Ruelle sempre vale para $\mathscr{L}_{f}$. O Teorema de Ruelle também vale para $\mathscr{L}_{f}$ quando $f \in W(\Omega)$, ver definições 4.1 e 4.2 e o Teorema 4.2.4.

Proposição 3.2.2. Seja $f \in C(\Omega)$ tal que o Teorema de Ruelle valha para $\mathscr{L}_{f}$, então o limite abaixo sempre existe $e$ independe de $x$,

$$
P(f)(x)=\lim _{n \rightarrow \infty} \frac{1}{n} \log \mathscr{L}_{f}^{n} \mathbf{1}(x)=\log \lambda_{f}
$$

Demonstração. Temos que

$$
\begin{aligned}
\lim _{n \rightarrow \infty} \frac{1}{n} \log \mathscr{L}_{f}^{n} \mathbf{1}(x) & =\lim _{n \rightarrow \infty} \frac{1}{n} \log \frac{\lambda_{f}^{n}}{\lambda_{f}^{n}} \mathscr{L}_{f}^{n} \mathbf{1}(x) \\
& =\lim _{n \rightarrow \infty} \frac{1}{n} \log \lambda_{f}^{n}+\lim _{n \rightarrow \infty} \frac{1}{n} \log \lambda_{f}^{-n} \mathscr{L}_{f}^{n} \mathbf{1}(x) \\
& =\log \lambda_{f}+\lim _{n \rightarrow \infty} \frac{1}{n} \log \lambda_{f}^{-n} \mathscr{L}_{f}^{n} \mathbf{1}(x) .
\end{aligned}
$$

Agora, como vale o item (b) de (3.2.1), temos que $\left\|\lambda_{f}^{-n} \mathscr{L}_{f}^{n} \mathbf{1}(x)-h_{f}(x)\right\|_{0} \rightarrow 0$, quando $n \rightarrow$ $\infty$, ou seja, $\lambda_{f}^{-n} \mathscr{L}_{f}^{n} \mathbf{1}(x)$ converge uniformemente para $h_{f}(x)$. Daí temos imediatamente que $\lim _{n \rightarrow \infty}(1 / n) \log \lambda_{f}^{-n} \mathscr{L}_{f}^{n} \mathbf{1}(x)=0$, donde segue o resultado.

Corolário 3.2.3. Seja $f$ um potencial em $C^{\gamma}(\Omega), 0<\gamma \leq 1$ ou $W(\Omega)$, então o limite abaixo sempre existe e independe de $x$,

$$
P(f)(x)=\lim _{n \rightarrow \infty} \frac{1}{n} \log \mathscr{L}_{f}^{n} \mathbf{1}(x)=\log \lambda_{f}
$$

Definição 3.2.4. Seja $f \in C^{\gamma}(\Omega)$, definimos a pressão topológica do potencial $f$ como sendo o número $P(f) \equiv \log \lambda_{f}$.

Feitas as considerações acima, temos o seguinte resultado:

Teorema 3.2.5. Seja $\mathcal{K} \subset C(\Omega)$ um subespaço invariante de $C(\Omega)$. Uma condição suficiente para que valha a analiticidade do funcional pressão é que o mapa $\mathcal{K} \ni f \mapsto \mathscr{L}_{f} \in \mathcal{K}$ seja analítico, que valha o Teorema de Ruelle para $\mathscr{L}_{f}$ e a presença do buraco espectral no operador de Ruelle $\mathscr{L}_{f}$.

No intuito de provar o Teorema 3.2.5 vamos usar o lema abaixo, que parece ser bem conhecido pela comunidade. Decidimos dar uma prova para este lema para manter o texto autocontido. 
Como mencionado anteriormente vamos usar expressão autovalor simples para nos referir a um autovalor $\lambda$ isolado de um operador $T: X \rightarrow X$ cuja a imagem do projetor espectral

$$
\pi_{T}=\int_{\partial D}(\lambda I-T)^{-1} d \lambda
$$

é um subespaço unidimensional de $X$.

Lema 3.2.6. Seja $T: X \rightarrow X$ um operador linear limitado possuindo um autovalor isolado simples $\lambda \in \mathbb{C}$. Seja $D$ um disco centrado em $\lambda$ tal que $D \cap \operatorname{spec}(T)=\{\lambda\}$. Então, existe uma vizinhança $U$ de $T$ em $\mathcal{L}(X, X)$ tal que o mapa $U \ni L \mapsto \lambda(L) \in \mathbb{C}$, onde $\lambda(L)$ é o único ponto em $\operatorname{spec}(L) \cap \operatorname{int}(D)$ está bem definido e além do mais esse mapa é analítico.

Corolário 3.2.7. Para qualquer $0<\gamma \leq 1$ fixado ambos os mapas

$$
C^{\gamma}(\Omega) \ni f \mapsto h_{f} \in C^{\gamma}(\Omega) \quad \text { e } \quad C^{\gamma}(\Omega) \ni f \mapsto \nu_{f} \in\left(C^{\gamma}(\Omega)\right)^{*}
$$

são analíticos.

Demonstração. Escolha $f$ arbitrariamente em $C^{\gamma}(\Omega)$. Sejam $U$ e $\tilde{U}$ vizinhanças de $\mathscr{L}_{f}$ e $\mathscr{L}_{f}^{*}$, respectivamente tais que para todo $\mathscr{L}_{g} \in U$ e $\mathscr{L}_{g}^{*} \in \tilde{U}$ as imagens dos projetores espectrais $\pi_{\mathscr{L}_{g}}$ e $\pi_{\mathscr{L}_{g}^{*}}$ sejam subespaços unidimensionais associados ao autovalor $\lambda_{g}$. Considere uma vizinhança $W \subset C^{\gamma}(\Omega)$ de $f$ tais que $\mathscr{L}_{g} \in U$ e $\mathscr{L}_{g}^{*} \in \tilde{U}$, respectivamente sempre que $g \in W$. Portanto temos que $\pi_{\mathscr{L}_{g}^{*}} \nu_{f}=C \cdot \nu_{g} \operatorname{Logo}$

$$
C=C \cdot \int_{\Omega} 1 d \nu_{g} \equiv C \cdot\left\langle\nu_{g}, 1\right\rangle=\left\langle\pi_{\mathscr{L}_{g}^{*}} \nu_{f}, 1\right\rangle
$$

donde segue que $\nu_{g}=\left\langle\pi_{\mathscr{L}_{g}^{*}} \nu_{f}, 1\right\rangle^{-1} \cdot \pi_{\mathscr{L}_{g}^{*}} \nu_{f}$. Sendo o lado direito da última expressão uma composição de funções analíticas segue que $g \mapsto \nu_{g}$ é analítica numa vizinhança de $f$. Por outro lado,$\pi_{\mathscr{L}_{g}} h_{f}=C \cdot h_{g}$. Integrando ambos os lados com relação a $\nu_{g}$ ficamos com $C=$ $C \cdot\left\langle\nu_{g}, h_{g}\right\rangle=\left\langle\nu_{g}, \pi_{\mathscr{L}_{g}} h_{f}\right\rangle$ e portanto $h_{g}=\left\langle\nu_{g}, \pi_{\mathscr{L}_{g}} h_{f}\right\rangle^{-1} \cdot \pi_{\mathscr{L}_{g}} h_{f}$ que composição de funções analíticas portanto $g \mapsto h_{g}$ é analítica numa vizinhança de $f$.

Agora estamos prontos para provar o principal resultado desta secção.

Prova do Teorema 3.2.5 A prova é baseada na analiticidade das funções $\mathcal{K} \ni f \mapsto \mathscr{L}_{f}$ e $U \ni T \mapsto \lambda(T) \in \mathbb{R}$. A analiticidade da primeira é conteúdo da Proposição 3.1.3. A analiticidade do segundo mapa segue da hipótese da existência do buraco espectral e do Lema 3.2.6. Para finalizar, como, por hipótese, o Teorema de Ruelle vale para $\mathscr{L}_{f}: \mathcal{K} \rightarrow \mathcal{K}$, ver (3.2.1), podemos escrever a pressão como $P(f)=\log \lambda_{f}$. Sendo assim, o lado direito da igualdade anterior é a composição das seguintes aplicações analíticas $\log , \mathcal{K} \ni f \mapsto \mathscr{L}_{f}$ e $U \ni T \mapsto \lambda(T) \in \mathbb{R}$.

Uma consequência imediata do Teorema acima é o seguinte corolário

Corolário 3.2.8. A função dada por $C^{\gamma}(\Omega) \ni f \mapsto P(f) \in \mathbb{R}$ é real analítica. 
Prova do Lema 3.2.6. O Argumento é baseado nas seguintes afirmações.

Afirmação 1. Existe uma vizinhança $U$ de $T \operatorname{em~} \mathcal{L}(X, X)$ tais que $\operatorname{spec}(L) \cap \partial D=\varnothing$ para todo $L \in U$. Provaremos esta afirmação por contradição. Suponha que para cada $n$ exista $L_{n} \in$ $B(T, 1 / n) \subset L(X, X)$ tal que $\lambda_{L_{n}} \in \operatorname{spec}\left(L_{n}\right) \cap \partial D$ e o operador $\lambda_{L_{n}} I-L_{n}$ não seja invertível. Como $\partial D$ é compacto podemos encontrar uma subsequência convergente $\left\{\lambda_{L_{n_{j}}}\right\} \subset \partial D$ tal que $\lambda_{L_{n_{j}}} \rightarrow \lambda_{L} \in \partial D$. Como $L_{n} \rightarrow T$ temos que $\left(\lambda_{L_{n_{j}}} I-L_{n_{j}}\right) \rightarrow\left(\lambda_{L} I-T\right)$ na topologia forte. Visto que $\left(\lambda_{L} I-T\right)$ é invertível e o espaço dos operadores lineares limitados invertíveis é aberto obtemos uma contradição.

Afirmação 2. Podemos encolher $U$ de modo que para todo $L \in U$ a interseção $\operatorname{spec}(L) \cap \operatorname{int}(D)$ seja um autovalor simples de $L$. De fato, para cada $L \in U$ seja $\pi_{L}$ o projetor espectral dado por

$$
\pi_{L}=\int_{\partial D}(\lambda I-L)^{-1} d \lambda
$$

Note que o mapa $U \ni L \mapsto \pi_{L} \in \mathscr{L}(X, X)$ é continuo, portanto pela Proposição (B.3.5) se necessário podemos encolher $U$ tal que para todo $L \in U$ a aplicação $\pi_{L}$ tenha o mesmo posto que $\pi_{T}$. Este fato juntamente como a observação B.3.2 implica que a porção do espectro de $L \in U$ que está em $\operatorname{int}(D)$ é não vazia, chame isso de $\Sigma(L)$. Defina $X_{\Sigma(L)} \equiv \pi_{L} X$ e $T_{\Sigma(L)}=\left.T\right|_{X_{\Sigma(L)}}$. É sabido de [33, p. 98] que $\operatorname{spec}\left(T_{\Sigma(L)}\right)=\Sigma(L)$. Se $\Sigma(L)$ não for um conjunto unitário, então $X_{\Sigma(L)}$ não é um subespaço unidimensional e portanto $1 \neq \operatorname{dim}\left(X_{\Sigma(L)}\right)=\operatorname{dim}\left(\pi_{T} X\right)=1$. Esta contradição mostra que existe um único autovalor simples $\lambda(L)$ of $L$ dentro de $D$. Usando as afirmações acima obtemos que a seguinte aplicação, $U \ni L \mapsto \lambda(L) \in \mathbb{C}$, onde $\lambda(L)$ é o único autovalor simples de $L$ dentro de $\operatorname{int}(D)$ está bem definida.

Passamos agora para a prova de que o mapa $U \ni L \mapsto \lambda(L) \in \mathbb{C}$ é analítico. Fixe $v \in X$ tal que $\pi_{L} v$ é não nulo e escolha $w \in X^{*}$ tal que $w\left(\pi_{L} v\right) \equiv\left\langle w, \pi_{L} v\right\rangle \neq 0$ (Hahn Banach) para todo $L$ em uma vizinhança suficientemente pequena de $T$. De acordo com a Proposição B.3.3 o operador $L$ comuta com $\pi_{L}$ nos fornecendo que $\left\langle w, \pi_{L}(L v)\right\rangle=\left\langle w, L \pi_{L}(v)\right\rangle=\lambda(L)\left\langle w, \pi_{L}(v)\right\rangle$ e consequentemente que

$$
\lambda(L)=\frac{\left\langle w, \pi_{L}(L v)\right\rangle}{\left\langle w, \pi_{L}(v)\right\rangle} .
$$

Da definição B.3.1 e da igualdade acima obtemos a analiticidade do mapa $U \ni L \mapsto \lambda(L) \in$ $\mathbb{C}$.

\subsection{A Derivada do Funcional Pressão}

Nesta seção provamos um resultado que generaliza a Proposição 3.2 .2 para potenciais contínuos sem assumir a validade do Teorema de Ruelle-Perron-Fröbenius.

Teorema 3.3.1. Se $f \in C(\Omega)$ então o limite

$$
P(f)(x)=\lim _{n \rightarrow \infty} \frac{1}{n} \log \mathscr{L}_{f}^{n} \mathbf{1}(x)
$$

existe para todo $x \in \Omega$ e independe de $x$. 
Demonstração. Para demonstrar este corolário basta provar que a sequência $\Phi_{n}: C(\Omega) \rightarrow C(\Omega)$ dada por

$$
\Phi_{n}(f)(x)=\frac{1}{n} \log \mathscr{L}_{f}^{n} \mathbf{1}(x)
$$

converge uniformemente nas partes compactas de $C(\Omega)$ para uma aplicação $\Phi: C(\Omega) \rightarrow C(\Omega)$ contínua. De fato, do Corolário 3.2.2 temos que $\Phi(f) \in C(\Omega)$ é uma função constante sempre que $f \in C^{\gamma}(\Omega), 0<\gamma \leq 1$. Segue da densidade de $C^{\gamma}(\Omega)$ em $C(\Omega)$ que dada $f \in C(\Omega)$ existe uma sequência $\left(f_{n}\right)_{n \in \mathbb{N}} \subset C^{\gamma}(\Omega)$ com $f_{n} \rightarrow f$. A partir de provada a continuidade uniforme de $\Phi$ obtemos que o limite $\Phi(f)=\lim _{n \rightarrow \infty} \Phi\left(f_{n}\right)$ está bem definido (independe da sequência) e a afirmação feita acima está provada.

Agora vamos mostrar que a sequência $\Phi_{n}: C(\Omega) \rightarrow C(\Omega)$ converge uniformemente nas partes compactas de $C(\Omega)$. Inicialmente repare que podemos identificar a sequência $\Phi_{n}: C(\Omega) \rightarrow$ $C(\Omega)$ com a sequência $\Phi_{n}: C(\Omega) \times \Omega \rightarrow \mathbb{R}$ onde $\Phi_{n}(f, x)=(1 / n) \log \mathscr{L}_{f}^{n} \mathbf{1}(x)$. Usando o Teorema da Convergência dominada é fácil ver que a derivada de Frechet de $\Phi_{n}: C(\Omega) \times \Omega \rightarrow \mathbb{R}$ com relação a $f$ é dada por

$$
\frac{\partial}{\partial f} \Phi_{n}(f, x) \cdot \varphi=\frac{1}{n} \frac{\int_{M^{n}}\left(S_{n} \varphi\right)(\mathbf{a} x) \exp \left(S_{n} f\right)(\mathbf{a} x) d \mu(\mathbf{a})}{\mathscr{L}_{f}^{n} \mathbf{1}(x)}
$$

Observe que,

$$
\left\|\frac{1}{n} \sum_{j=0}^{n-1} \varphi \circ \sigma^{j}\right\|_{0} \leq \frac{1}{n} \sum_{j=0}^{n-1}\left\|\varphi \circ \sigma^{j}\right\|_{0} \leq \frac{1}{n} \sum_{j=0}^{n-1}\|\varphi\|_{0}=\|\varphi\|_{0} .
$$

Esta desigualdade implica na seguinte estimativa

$$
\begin{aligned}
\left|\frac{\partial}{\partial f} \Phi_{n}(f, x) \cdot \varphi\right| & =\left|\frac{\int_{M^{n}} \frac{1}{n}\left(S_{n} \varphi\right)(\mathbf{a} x) \exp \left(S_{n} f\right)(\mathbf{a} x) d \mu(\mathbf{a})}{\mathscr{L}_{f}^{n} \mathbf{1}(x)}\right| \\
& \leq \frac{\int_{M^{n}}\left|\frac{1}{n}\left(S_{n} \varphi\right)(\mathbf{a} x) \exp \left(S_{n} f\right)(\mathbf{a} x)\right| d \mu(\mathbf{a})}{\left|\int_{M^{n}} \exp \left(S_{n} f\right)(\mathbf{a} x) d \mu(\mathbf{a})\right|} \\
& \leq\|\varphi\|_{0}
\end{aligned}
$$

para todo $x \in \Omega$. Tomando o supremo sobre todos os $x \in \Omega$ ficamos com

$$
\left\|\frac{\partial}{\partial f} \Phi_{n}(f, x) \cdot \varphi\right\|_{0} \leq\|\varphi\|_{0}
$$

para todo $n \in \mathbb{N}$. Desta desigualdade concluímos que $\left\|\frac{\partial}{\partial f} \Phi_{n}(f)\right\| \leq 1$ (norma de operador).

Fixe $f$ e $\tilde{f}$ em $\underset{\tilde{f}}{C}(\Omega)$ e defina para cada $n \in \mathbb{N}$ a seguinte função $\hat{\Phi}_{n}(t)=\Phi_{n}(\gamma(t), x)$, onde $\gamma(t)=t f+(1-t) \tilde{f} \operatorname{com} 0 \leq t \leq 1$. Obviamente $\hat{\Phi}_{n}$ é uma função diferenciável vista como uma 
aplicação de $[0,1]$ em $\mathbb{R}$. Além do mais temos $\left|\hat{\Phi}_{n}(1)-\hat{\Phi}_{n}(0)\right|=\left|\frac{d}{d t} \hat{\Phi}_{n}(\hat{t})(1-0)\right|$ para algum $\hat{t} \in(0,1)$. Usando a estimativa da norma da derivada de Frechet de $\Phi_{n}(f, x)$ temos

$$
\left|\Phi_{n}(f, x)-\Phi_{n}(\tilde{f}, x)\right|=\left|\frac{\partial}{\partial f} \Phi_{n}(f, x)(f-\tilde{f})\right| \leq\|f-\tilde{f}\|_{0} .
$$

Consequentemente a sequência $\Phi_{n}: C(\Omega) \rightarrow C(\Omega)$ é uniformemente equicontínua. Devido a Proposição 3.2 .2 a sequência $\Phi_{n}$ converge pontualmente no conjunto denso $C^{\gamma}(\Omega) \subset C(\Omega)$. Além disso para cada $f \in C(\Omega)$ o conjunto $\left\{\Phi_{n}(f), n \in \mathbb{N}\right\}$ é pontualmente limitado, isto é, existe $M(f)$ tal que $\left|\Phi_{n}(f)\right| \leq M(f)$. De fato, usando a desigualdade triangular, a última desigualdade acima e que existe $\lim _{n \rightarrow \infty} \Phi_{n}(1)$ temos para quaisquer $f \in C^{\gamma}(\Omega)$ que

$$
\begin{aligned}
\left|\Phi_{n}(f)\right|=\left|\Phi_{n}(f)-\Phi_{n}(1)\right|+\left|\Phi_{n}(1)\right| & \leq\|f-1\|_{\infty}+\left|\Phi_{n}(1)\right| \\
& \leq\|f-1\|_{\infty}+\sup _{n \in \mathbb{N}}\left|\Phi_{n}(1)\right| \\
& \equiv M(f) .
\end{aligned}
$$

Agora estamos em condições de aplicar o Teorema de Arzelà-Àscoli para garantir que existe uma subsequência $\left(\Phi_{n_{k}}\right)_{k \in \mathbb{N}}$ que converge nas partes compactas de $C(\Omega)$ para uma função contínua $\Phi: C(\Omega) \rightarrow C(\Omega)$.

Afirmamos que $\Phi_{n}(f) \rightarrow \Phi(f)$, quando $n \rightarrow \infty$. De fato, dado $\varepsilon>0$ seja $g \in C^{\gamma}(\Omega)$ tal que $\|f-g\|_{0}<\varepsilon$. Tome $n_{k}$ e $n$ suficientemente grandes de forma que valham as seguintes desigualdades $\left\|\Phi_{n_{k}}(f)-\Phi(f)\right\|_{\infty}<\varepsilon,\left\|\Phi_{n}(g)-\Phi_{n_{k}}(g)\right\|_{\infty}<\varepsilon$ e $\left\|\Phi_{n_{k}}(g)-\Phi_{n_{k}}(f)\right\|_{\infty}<\varepsilon$. Com estas escolhas de $g, n$ e $n_{k}$ temos da desigualdades triangular e da desigualdade (3.9) que

$$
\begin{aligned}
\left\|\Phi_{n}(f)-\Phi(f)\right\|_{\infty} & \leq\left\|\Phi_{n}(f)-\Phi_{n_{k}}(f)+\Phi_{n_{k}}(f)-\Phi(f)\right\|_{\infty} \\
& \leq\left\|\Phi_{n}(f)-\Phi_{n_{k}}(f)\right\|_{\infty}+\left\|\Phi_{n_{k}}(f)-\Phi(f)\right\|_{\infty} \\
& <\left\|\Phi_{n}(f)-\Phi_{n_{k}}(f)\right\|_{\infty}+\varepsilon \\
& \leq\left\|\Phi_{n}(f)-\Phi_{n}(g)\right\|_{\infty}+\left\|\Phi_{n}(g)-\Phi_{n_{k}}(f)\right\|_{\infty}+\varepsilon \\
& <\left\|\Phi_{n}(g)-\Phi_{n_{k}}(f)\right\|_{\infty}+2 \varepsilon \\
& \leq\left\|\Phi_{n}(g)-\Phi_{n_{k}}(g)\right\|_{\infty}+\left\|\Phi_{n_{k}}(g)-\Phi_{n_{k}}(f)\right\|_{\infty}+2 \varepsilon \\
& <4 \varepsilon .
\end{aligned}
$$

Agora note que que 3.9 permanece após passagem ao limite, o que implica que $\Phi$ tem estensão única a fecho uniforme $C^{\gamma}(\Omega)=C(\Omega)$. Ver o parágrafo 4 do Capítulo 7 de [31].

Corolário 3.3.2. Para todo $0<\gamma \leq 1, \nu_{f}$ é uma função fracamente continua de $f \in C^{\gamma}(\Omega)$, isto é, se $\left\|f-f_{n}\right\|_{\gamma} \rightarrow 0$ então

$$
\lim _{n \rightarrow \infty} \int \varphi d \nu_{f_{n}}=\int \varphi d \nu_{f}, \quad \forall \varphi \in C(\Omega) .
$$

Demonstração. Seja $\left\{f_{n}\right\} \subset C^{\gamma}(\Omega)$ uma sequência tal que $f_{n} \rightarrow f \in C^{\gamma}(\Omega)$. Suponha que $\nu_{f_{n}}$ não convirja fracamente para $\nu_{f}$. Pela compacidade e metrizabilidade de $\mathcal{M}(\Omega)=C(\Omega)^{*}$ 
( $\Omega$ é espaço métrico compacto) podemos assumir sem perda de generalidade que $\nu_{f_{n}}$ converge fracamente para uma probabilidade $\nu$. Então, como os mapas $f \mapsto \mathscr{L}_{f}$ e $f \rightarrow \lambda_{f}$ são contínuos,

$$
\mathscr{L}_{f}^{*} \nu=\lim \mathscr{L}_{f_{n}}^{*} \nu_{n}=\lim \lambda_{f_{n}} \nu_{f_{n}}=\lambda_{f} \nu
$$

Portanto, $\nu \in C(\Omega)^{*}$ é uma automedida de $\mathscr{L}_{f}^{*}: C^{\gamma}(\Omega)^{*} \rightarrow C^{\gamma}(\Omega)^{*}$ associada ao autovalor $\lambda_{f}$. Como o autoespaço de $\mathscr{L}_{f}^{*}$ associado ao autovalor $\lambda_{f}$ é unidimensional, segue que $\nu$ é múltiplo de $\nu_{f}$. Como $\nu$ e $\nu_{f}$ são probabilidades temos $\nu=\nu_{f}$.

Corolário 3.3.3. Seja $f \in C^{\gamma}(\Omega), 0<\gamma \leq 1$, então

$$
\lim _{n \rightarrow \infty}\left\|\frac{1}{n} \frac{\mathscr{L}_{f}^{n}\left(S_{n} \varphi\right)}{\mathscr{L}_{f}^{n} \mathbf{1}}-\int \varphi h_{f} d \nu_{f}\right\|_{0}=0
$$

para toda $\varphi \in C(\Omega)$, além disso esta convergência é uniforme.

Demonstração. Fazendo manipulações algébricas simples temos

$$
\begin{aligned}
\left\|\frac{1}{n} \frac{\mathscr{L}_{f}^{n}\left(S_{n} \varphi\right)}{\mathscr{L}_{f}^{n} \mathbf{1}}-\int \varphi h_{f} d \nu_{f}\right\|_{0} & =\left\|\frac{\lambda_{f}^{n}}{\mathscr{L}_{f}^{n} \mathbf{1}} \frac{1}{n} \lambda_{f}^{-n} \mathscr{L}_{f}^{n}\left(S_{n} \varphi\right)-\int \varphi h_{f} d \nu_{f}\right\|_{0} \\
& \leq \sup _{n \in \mathbb{N}}\left\|\frac{\lambda_{f}^{n}}{\mathscr{L}_{f}^{n} \mathbf{1}}\right\|_{0}\left\|\frac{1}{n} \lambda_{f}^{-n} \mathscr{L}_{f}^{n}\left(S_{n} \varphi\right)-\left(\lambda_{f}^{-n} \mathscr{L}_{f}^{n} \mathbf{1}\right) \int \varphi h_{f} d \nu_{f}\right\|_{0} .
\end{aligned}
$$

Portanto para provar a existência do limite afirmada no enunciado basta analisar as seguintes quantidades

$$
\sup _{n}\left\|\frac{\lambda_{f}^{n}}{\mathscr{L}_{f}^{n} \mathbf{1}}\right\|_{0}, \quad\left(\lambda_{f}^{-n} \mathscr{L}_{f}^{n} \mathbf{1}\right) \int \varphi h_{f} d \nu_{f} \text { e } \frac{1}{n} \lambda_{f}^{-n} \mathscr{L}_{f}^{n}\left(S_{n} \varphi\right) .
$$

As duas primeiras das quantidades acimas são relativamente simples de se estudar utilizando diretamente o Teorema de Ruelle-Perron-Fröbenius (Teorema 2.3.4). De fato, segue do Teorema de Ruelle que $\lambda_{f}^{-n} \mathscr{L}_{f}^{n} \mathbf{1} \stackrel{\|\cdot\|_{0}}{\longrightarrow} h_{f}$, isso fornece imediatamente que

$$
\left(\lambda_{f}^{-n} \mathscr{L}_{f}^{n} \mathbf{1}\right) \int \varphi h_{f} d \nu_{f} \stackrel{\|\cdot\|_{0}}{\longrightarrow} h_{f} \int \varphi h_{f} d \nu_{f}
$$

Lembrando que $h_{f}$ é estritamente positiva, e portanto uniformemente afastada de 0 pois é contínua no compacto $\Omega$, temos que $\lambda_{f}^{n} / \mathscr{L}_{f}^{n} \mathbf{1} \stackrel{\|\cdot\|_{0}}{\longrightarrow} 1 / h_{f}$, como $1 / h_{f}$ também é positiva e uniformemente afastada de 0 temos

$$
\sup _{n}\left\|\frac{\lambda_{f}^{n}}{\mathscr{L}_{f}^{n} \mathbf{1}}\right\|_{0}<\infty
$$

A última das quantidades acima é consideravelmente mais complicada de ser analisada e faremos seu estudo através de uma série de afirmações. 
Afirmação 1. Para qualquer $\varphi \in C(\Omega)$ e todo $n \in \mathbb{N}$ temos que

$$
\mathscr{L}_{f}^{n}\left(S_{n} \varphi\right)=\sum_{j=0}^{n-1} \mathscr{L}_{f}^{n-j}\left(\varphi \mathscr{L}_{f}^{j} \mathbf{1}\right)
$$

De fato, é imediato verificar usando a definição do operador de Ruelle que

$$
\mathscr{L}_{f}^{n}\left(\varphi \circ \sigma^{n}\right)=\varphi \mathscr{L}_{f}^{n} \mathbf{1}
$$

Daí segue que

$$
\begin{aligned}
\mathscr{L}_{f}^{n}\left(S_{n} \varphi\right) & =\mathscr{L}_{f}^{n-1}\left[\mathscr{L}_{f}\left\{\varphi+\varphi \circ \sigma+\varphi \circ \sigma^{2}+\ldots+\varphi \circ \sigma^{n-1}\right\}\right] \\
& =\mathscr{L}_{f}^{n}(\varphi)+\mathscr{L}_{f}^{n-1}\left(\mathscr{L}_{f}(\varphi \circ \sigma)\right)+\mathscr{L}_{f}^{n-2}\left(\mathscr{L}_{f}^{2} \varphi \circ \sigma^{2}\right)+\ldots+\mathscr{L}_{f}\left(\mathscr{L}_{f}^{n-1} \varphi \circ \sigma^{n-1}\right) \\
& =\mathscr{L}_{f}^{n}(\varphi)+\mathscr{L}_{f}^{n-1}\left(\varphi \mathscr{L}_{f} \mathbf{1}\right)+\mathscr{L}_{f}^{n-2}\left(\varphi \mathscr{L}_{f}^{2} \mathbf{1}\right)+\ldots+\mathscr{L}_{f}\left(\varphi \mathscr{L}_{f}^{n-1} \mathbf{1}\right) \\
& =\sum_{j=0}^{n-1} \mathscr{L}_{f}^{n-j}\left(\varphi \mathscr{L}_{f}^{j} \mathbf{1}\right)
\end{aligned}
$$

provando a Afirmação 1. Note que da Afirmação 1 temos imediatamente que

$$
\lambda_{f}^{-n} \mathscr{L}_{f}^{n}\left(S_{n} \varphi\right)=\sum_{j=0}^{n-1} \lambda_{f}^{-(n-j)} \mathscr{L}_{f}^{n-j}\left(\varphi \lambda_{f}^{-j} \mathscr{L}_{f}^{j} \mathbf{1}\right)
$$

Afirmação 2. Para qualquer $\varphi \in C(\Omega)$ existe o seguinte limite

$$
\lim _{n \rightarrow \infty}\left\|\frac{1}{n}\left(\lambda_{f}^{-n} \mathscr{L}_{f}^{n}\left(S_{n} \varphi\right)-\sum_{j=0}^{n-1} \lambda_{f}^{-(n-j)} \mathscr{L}_{f}^{n-j} \varphi h_{f}\right)\right\|_{0}=0 .
$$

Para mostrar que esta afirmação é verdadeira usamos a identidade obtida e a limitação do operador de Ruelle na norma de operadores em $C(\Omega)$ como segue

$$
\begin{aligned}
\left\|\frac{1}{n}\left\{\lambda_{f}^{-n} \mathscr{L}_{f}^{n}\left(S_{n} \varphi\right)-\sum_{j=0}^{n-1} \lambda_{f}^{-(n-j)} \mathscr{L}_{f}^{-(n-j)} \varphi h_{f}\right\}\right\|_{0} \\
=\left\|\frac{1}{n}\left\{\sum_{j=0}^{n-1} \lambda_{f}^{-(n-j)} \mathscr{L}_{f}^{n-j}\left(\varphi \lambda_{f}^{-j} \mathscr{L}_{f}^{j} \mathbf{1}\right)-\sum_{j=0}^{n-1} \lambda_{f}^{-(n-j)} \mathscr{L}_{f}^{n-j} \varphi h_{f}\right\}\right\|_{0} \\
=\left\|\frac{1}{n} \sum_{j=0}^{n-1} \lambda_{f}^{-(n-j)} \mathscr{L}_{f}^{n-j}\left(\varphi \lambda_{f}^{-j} \mathscr{L}_{f}^{j} \mathbf{1}-\varphi h_{f}\right)\right\|_{0} \\
\leq \frac{1}{n} \sum_{j=0}^{n-1}\left\|\lambda_{f}^{-(n-j)} \mathscr{L}_{f}^{n-j}\left(\varphi \lambda_{f}^{-j} \mathscr{L}_{f}^{j} \mathbf{1}-\varphi h_{f}\right)\right\|_{0} \\
\leq \frac{1}{n} \sum_{j=0}^{n-1}\left\|\lambda_{f}^{-(n-j)} \mathscr{L}_{f}^{n-j}\right\|_{\mathscr{L}(C(\Omega), C(\Omega))}\left\|\varphi \lambda_{f}^{-j} \mathscr{L}_{f}^{j} \mathbf{1}-\varphi h_{f}\right\|_{0} \\
\leq \frac{M}{n} \sum_{j=0}^{n-1}\left\|\varphi \lambda_{f}^{-j} \mathscr{L}_{f}^{j} \mathbf{1}-\varphi h_{f}\right\|_{0} \rightarrow 0
\end{aligned}
$$


quando $n \rightarrow \infty$. Note que o último termo da desigualdade acima converge a 0 pois é uma soma no sentido de Cesáro associada a sequência $\varphi \lambda_{f}^{-j} \mathscr{L}_{f}^{j} \mathbf{1}-\varphi h_{f}$, que converge a 0 na norma uniforme pelo Teorema de Ruelle-Perron-Fröbenius.

Afirmação 3. Para toda $\varphi \in C(\Omega)$ existe o seguinte limite

$$
\lim _{n \rightarrow \infty}\left\|\frac{1}{n} \sum_{j=0}^{n-1} \lambda_{f}^{-(n-j)} \mathscr{L}_{f}^{n-j} \varphi h_{f}-h_{f} \int \varphi h_{f} d \nu_{f}\right\|_{0}=0 .
$$

Defina $A_{n, j}:=\lambda_{f}^{-(n-j)} \mathscr{L}_{f}^{n-j} \varphi h_{f}$ e $B:=h_{f} \int \varphi h_{f} d \nu_{f}$. Pelo Teorema de Ruelle-Perron-Fröbenius temos para qualquer $j \in \mathbb{N}$ fixado que $\lim _{n \rightarrow \infty}\left\|A_{n, j}-B\right\|_{0}=0$. Por outro lado, temos da convergência da desigualdade triangular e da convergência no sentido de Cesaro que

$$
\begin{aligned}
\left\|\frac{1}{n} \sum_{j=0}^{n-1} A_{n, j}-B\right\|_{0} & =\left\|\frac{1}{n} \sum_{j=0}^{n-1} A_{n, j}-\frac{n}{n} B\right\|_{0}=\frac{1}{n}\left\|\sum_{j=0}^{n-1} A_{n, j}-n B\right\|_{0} \\
& =\frac{1}{n}\left\|\sum_{j=0}^{n-1}\left(A_{n, j}-B\right)\right\|_{0} \leq \frac{1}{n} \sum_{j=0}^{n-1}\left\|A_{n, j}-B\right\|_{0} \longrightarrow 0
\end{aligned}
$$

quando $n \rightarrow \infty$. O que prova a Afirmação 3 e encerra a prova do Corolário.

Corolário 3.3.4. Para qualquer $0<\gamma \leq 1$ fixado e $f \in C^{\gamma}(\Omega)$ temos que a derivada de Frechet do funcional pressão $P: C^{\gamma}(\Omega) \rightarrow \mathbb{R}$ é dada por

$$
P^{\prime}(f) \varphi=\int \varphi h_{f} d \nu_{f}
$$

Demonstração. Fixe $x \in \Omega$ e considere o funcional $\Phi_{n}: C^{\gamma}(\Omega) \rightarrow \mathbb{R}$ como definido no Teorema 3.3.1, isto é,

$$
\Phi_{n}(f) \equiv \frac{1}{n} \log \left(\mathscr{L}_{f}^{n} \mathbf{1}\right)(x)
$$

Como já foi visto no Teorema 3.3.1, a derivada de Frechet de $P_{n}$ aplicada em $\varphi \in C^{\gamma}(\Omega)$ é dada por

$$
\Phi_{n}^{\prime}(f) \varphi=\frac{1}{n} \frac{\mathscr{L}_{f}^{n}\left(S_{n} \varphi\right)}{\mathscr{L}_{f}^{n} \mathbf{1}},
$$

Já que a pressão em $C^{\gamma}(\Omega)$ é analítica segue do corolário anterior que

$$
P^{\prime}(f) \varphi=\lim _{n \rightarrow \infty} \Phi_{n}^{\prime}(f) \varphi=\int \varphi h_{f} d \nu_{f}
$$




\section{Capítulo 4}

\section{O TeOrema de Ruelle no ESPAÇO DE WALTERS}

\subsection{O Operador de Ruelle no EspaÇo de Walters}

Para simplificar a notação, se $f \in C(\Omega)$ e $x, y \in \Omega$, escrevemos

$$
S_{n} f(x) \equiv f(x)+f(\sigma(x))+\ldots+f\left(\sigma^{n-1}(x)\right) \quad \text { e } \quad d_{n}(x, y) \equiv \max _{0 \leq k<n} d_{\Omega}\left(\sigma^{k} x, \sigma^{k} y\right) .
$$

Definição 4.1.1. Diremos que uma função contínua $f: \Omega \rightarrow \mathbb{R}$ satisfaz a condição de Walters se dado $\varepsilon>0$ existe $\eta>0$ tal que

$$
\forall n \geq 1, \forall x, y \in \Omega, \quad d_{n}(x, y) \leq \eta \quad \Longrightarrow \quad\left|S_{n} f(x)-S_{n} f(y)\right| \leq \varepsilon .
$$

O espaço de todas as funções satisfazendo a condição de Walters é denotado por $W(\Omega)$.

A seguir abordaremos outra alternativa de se generalizar a condição de Walters para o contexto de espaço de estados compactos.

Definição 4.1.2. Seja $f: \Omega \rightarrow \mathbb{R}$ uma função contínua, defina $C_{f}(x, y)$ por

$$
C_{f}(x, y)=\sup _{n \geq 1} \sup _{\mathbf{a} \in M^{n}} S_{n} f(\mathbf{a} x)-S_{n} f(\mathbf{a} y) .
$$

Dizemos que $f$ satisfaz a condição fraca de Walters se $C_{f}(x, y) \rightarrow 0$ quando $d(x, y) \rightarrow 0$.

Exemplo. Considere o espaço métrico $(M, d)$ onde $M=[0,1]$ e $d=|\cdot|$ (valor absoluto). Agora seja $f$ o potencial definido em $\Omega=M^{\mathbb{N}}$ dado por $f(x)=x_{1}$, isto é, $f$ depende apenas da primeira coordenada. Afirmamos que $f$ satisfaz a condição fraca de Walters mas não satisfaz a condição de Walters (forte). De fato, temos que $S_{n} f(\mathbf{a} x)=S_{n} f(\mathbf{a} y)=\sum_{i=1}^{n} a_{i}$ para qualquer $\mathbf{a}=\left(a_{1}, \ldots, a_{n}\right) \in M^{n}$. Portanto $f$ satisfaz claramente a condição fraca de Walters. Note que $f$ não satisfaz a condição de Walters. De fato, considere $x=(0,0 \ldots)$ o vetor nulo e 
$y=(\eta, \eta, \ldots)$ para $\eta$ pequeno. Temos que $d_{\Omega}(x, y)=d_{n}(x, y)=\eta$. Por outro lado, $S_{n} f(x)=0$ e $S_{n} f(y)=n \cdot \eta$, portanto

$$
\left|S_{n} f(x)-S_{n} f(y)\right|=n \cdot \eta
$$

Segue dai que $f$ não satisfaz a condição de Walters. Por outro lado, a implicação oposta é sempre verdadeira.

Proposição 4.1.3. Se $f \in C(\Omega)$ satisfaz a condição de Walters então $f$ satisfaz a condição fraca de Walters.

Demonstração. Seja $f \in W(\Omega)$, então por definição dado $\varepsilon>0$ existe $\eta>0$ tal que $\forall n \geq$ $1, \forall z, w \in \Omega \operatorname{com} d_{n}(z, w) \leq \eta \operatorname{nos}$ fornece $\left|S_{n} f(z)-S_{n} f(w)\right| \leq \varepsilon$. Note que $d_{n}(\mathbf{a} x, \mathbf{a} y) \leq d(x, y)$ para qualquer $\mathbf{a} \in M^{n}$. Portanto $d(x, y) \leq \eta \Rightarrow d_{n}(\mathbf{a} x, \mathbf{a} y) \leq \eta \Rightarrow\left|S_{n} f(\mathbf{a} x)-S_{n} f(\mathbf{a} y)\right| \leq$ $\varepsilon, \quad \forall \mathbf{a} \in M^{n}, \quad \forall n \geq 1$. Tomando o supremo sobre todos os $\mathbf{a} \in M^{n}$ e $n \geq 1$ o resultado segue.

O espaço $W(\Omega)$ é claramente um subespaço vetorial de $C(\Omega)$. Denote por $S$ a constante de expansividade do shift $\sigma$. Em [5] é provado que para todo $s \in(0, S)$ a seguinte expressão

$$
\|f\|_{W_{s}}=2\|f\|_{0}+\sup _{n \geq 1} \max _{d_{n}(x, y) \leq s}\left|S_{n} f(x)-S_{n} f(y)\right|
$$

define uma família de normas equivalentes e que $\left(W(\Omega),\|\cdot\|_{W_{s}}\right)$ é um espaço de Banach. Visto que a família de normas $\left(\|\cdot\|_{W_{s}}\right)_{0<s<S}$ fornece a mesmas topologia, podemos, sem perda de generalidade, fixar um $s \in(0, S)$ particular e desenvolver a teoria com a norma $\|\cdot\|_{W} \equiv\|\cdot\|_{W_{s}}$. No intuito de provar um dos principais resultados deste trabalho, precisamos de uma estrutura extra de álgebra de Banach para $W(\Omega)$. Este é o conteúdo do próximo lema.

Lema 4.1.4. O espaço $W(\Omega)$ com a norma $\|\cdot\|_{W}$ é uma álgebra de Banach sobre $\mathbb{R}$, isto é, $W(\Omega)$ é um espaço de Banach real e para todos $f, g \in W(\Omega)$ temos $\|f g\|_{W} \leq\|f\|_{W}\|g\|_{W}$.

Demonstração. Sejam $f, g \in W(\Omega)$ e defina $I \equiv I(f, g)$ pondo

$$
\begin{aligned}
I & \equiv \sup _{n \geq 1} \max _{d_{n}(x, y) \leq s}\left|S_{n}(f g)(x)-S_{n}(f g)(y)\right| \\
& =\sup _{n \geq 1} \max _{d_{n}(x, y) \leq s}\left|\sum_{j=1}^{n-1}(f g) \circ \sigma^{j}(x)-\sum_{j=1}^{n-1}(f g) \circ \sigma^{j}(y)\right| \\
& =\sup _{n \geq 1} \max _{d_{n}(x, y) \leq s} \mid \sum_{j=1}^{n-1}(f g) \circ \sigma^{j}(x)-\sum_{j=1}^{n-1} f \circ \sigma^{j}(x) g \circ \sigma^{j}(y) \\
& \quad+\sum_{j=1}^{n-1} f \circ \sigma^{j}(x) g \circ \sigma^{j}(y)-\sum_{j=1}^{n-1}(f g) \circ \sigma^{j}(y) \mid .
\end{aligned}
$$


Aplicando a desigualdade triangular obtemos que

$$
\begin{aligned}
I \leq \sup _{n \geq 1} \max _{d_{n}(x, y) \leq s}\left|\sum_{j=1}^{n-1} f \circ \sigma^{j}(x)\left[g \circ \sigma^{j}(x)-g \circ \sigma^{j}(y)\right]\right| \\
\quad+\sup _{n \geq 1} \max _{d_{n}(x, y) \leq s}\left|\sum_{j=1}^{n-1} g \circ \sigma^{j}(y)\left[f \circ \sigma^{j}(x)-f \circ \sigma^{j}(y)\right]\right| \\
\leq\|f\|_{0} \cdot \sup _{n \geq 1} \max _{d_{n}(x, y) \leq s}\left|S_{n} g(x)-S_{n} g(y)\right|+\|g\|_{0} \cdot \sup _{n \geq 1} \max _{d_{n}(x, y) \leq s}\left|S_{n} f(x)-S_{n} f(y)\right| .
\end{aligned}
$$

Segue da desigualdade obtida acima que

$$
\|f g\|_{W} \leq 2\|f g\|_{0}+\|f\|_{0} \sup _{n \geq 1} \max _{d_{n}(x, y) \leq s}\left|S_{n} g(x)-S_{n} g(y)\right|+\|g\|_{0} \sup _{n \geq 1} \max _{d_{n}(x, y) \leq s}\left|S_{f}(x)-S_{n} f(y)\right| .
$$

Por outro lado, segue da definição de $\|\cdot\|_{W}$ que

$$
\begin{aligned}
\|f\|_{W} \cdot\|g\|_{W}=4\|f\|_{0}\|g\|_{0} & +2\|f\|_{0} \cdot \sup _{n \geq 1} \max _{d_{n}(x, y) \leq s}\left|S_{n} g(x)-S_{n} g(y)\right| \\
& +2\|g\|_{0} \cdot \sup _{n \geq 1} \max _{d_{n}(x, y) \leq s}\left|S_{f}(x)-S_{n} f(y)\right| \\
& +\sup _{n \geq 1} \max _{d_{n}(x, y) \leq s}\left|S_{n} g(x)-S_{n} g(y)\right| \cdot \sup _{n \geq 1} \max _{d_{n}(x, y) \leq s}\left|S_{n} f(x)-S_{n} f(y)\right| .
\end{aligned}
$$

Esta identidade e as estimativas anteriores implicam que $\|f g\|_{W} \leq\|f\|_{W} \cdot\|g\|_{W}$.

Proposição 4.1.5. Se $f \in W(\Omega)$, então $\mathscr{L}_{f}(W(\Omega)) \subset W(\Omega)$.

Demonstração. Afirmamos que para qualquer $a \in M$ fixado, se $f \in W(\Omega)$ então a função $x \mapsto f(a x)$ também pertence a $W(\Omega)$. De fato, dado $\varepsilon>0$ escolha $\eta>0$ tal que a condição de Walters 4.1) seja satisfeita por $f$. Note que $d_{n}(x, y) \leq \delta \Rightarrow d_{n}(a x, a y) \leq d_{n}(x, y) \leq \eta$. Pela exposto acima temos que $d_{n}(x, y) \leq \eta$ implica $\left|S_{n} f(a x)-S_{n} f(a y)\right| \leq \varepsilon$ para todo $n>0$ e portanto a afirmação está provada. O próximo passo é provar que a função $r: \Omega \rightarrow \mathbb{R}$ dada por $r(x)=\int_{M} h(a x) d \mu(a)$ pertence a $W(\Omega)$ sempre que $h \in W(\Omega)$. Segue da afirmação anterior que $x \mapsto h(a x)$ is in $W(\Omega)$ visto que $h \in W(\Omega)$. Dado $\varepsilon>0$ podemos escolher $\eta>0$ tal que a condição (4.1) seja satisfeita por $x \mapsto h(a x)$. Como $d_{n}(x, y) \leq \eta \Rightarrow d_{n}(a x, a y) \leq \eta$, a condição de Walters para $r$ segue da desigualdade abaixo

$$
\left|S_{n} r(x)-S_{n} r(y)\right| \leq \int_{M}\left|S_{n} h(a x)-S_{n} h(a y)\right| d \mu(a) \leq \varepsilon \cdot \mu(M) .
$$

Por hipótese $f \in W(\Omega)$, e como os espaço de Walters é uma álgebra de Banach temos que $\exp (f) \in W(\Omega)$. Pela mesma razão, para qualquer $\varphi \in W(\Omega)$ temos que $\varphi \cdot \exp (f) \in W(\Omega)$. Com foi argumentado acima, para qualquer $a \in M$ fixado, a função $x \mapsto \varphi(a x) \cdot \exp (f(a x))$ pertence a $W(\Omega)$. Usando o resultado acima que foi provado para a função $r$, com $h(x)=$ $\varphi(x) \exp (f(x))$, temos que

$$
x \mapsto \int_{M} \varphi(a x) \exp (f(a x)) d \mu(a) \equiv \mathscr{L}_{f}(\varphi)(x)
$$

está no espaço de Walters para qualquer $\varphi \in W(\Omega)$ o que finaliza a prova. 


\subsection{O TeOrema de Ruelle no EspaÇo de Walters}

A prova dessa versão do Teorema de Ruelle é inspirada na prova original dada por Walters em [50]. Para provar o Teorema de Ruelle vamos concentrar nossa atenção numa certa subclasse de $C(\Omega)$ a qual é dada por

$$
G_{0}(\Omega)=\left\{g \in C(\Omega): g>0 \text { e } \int_{M} g(a x) d \mu(a)=1, \forall x \in \Omega\right\} .
$$

Se $f: \Omega \rightarrow \mathbb{R}$ é um potêncial dado por $f=\log g$, onde $g \in G_{0}(\Omega)$, então a condição fraca de Walters para $f$ pode ser refraseada em termos de $g$ do seguinte modo, dizendo que

$$
D_{g}(x, y)=\sup _{n \geq 1} \sup _{\mathbf{a} \in M^{n}} \prod_{i=0}^{n-1} \frac{g\left(\sigma^{i} \mathbf{a} x\right)}{g\left(\sigma^{i} \mathbf{a} y\right)}
$$

existe, é limitado por uma constante $D_{g}$ e $D_{g}(x, y) \rightarrow 1$ quando $d_{\Omega}(x, y) \rightarrow 0$. Equivalentemente:

$$
D_{g}^{\star}(x, y)=\sup _{n \geq 1} \sup _{\mathbf{a} \in M^{n}}\left|\prod_{i=0}^{n-1} \frac{g\left(\sigma^{i} \mathbf{a} x\right)}{g\left(\sigma^{i} \mathbf{a} y\right)}-1\right| \leq D_{g}-1
$$

para todo $x, y$ com $d_{\Omega}(x, y)<\varepsilon_{0}, D_{g}^{\star}(x, y) \rightarrow 0$ quando $d_{\Omega}(x, y) \rightarrow 0$.

Teorema 4.2.1. Seja $g \in G_{0}(\Omega)$ um potencial tal que $\log g$ satisfaz a condição fraca de Walters. Então existe uma medida de probabilidade $\nu: \mathscr{B}(\Omega) \rightarrow[0,1]$ tal que

$$
\mathscr{L}_{\log g}^{n} \varphi \stackrel{\|\cdot\|_{0}}{\longrightarrow} \nu(\varphi)
$$

para toda $\varphi \in C(\Omega)$. Além disso $\nu$ é a única probabilidade satisfazendo $\mathscr{L}_{\log g}^{*} \nu=\nu$.

Demonstração. Para provar este teorema seguiremos de maneira bastante próxima os argumentos fornecidos em [48]. Como sempre os pequenos problemas que aparecem, devido a mudança de contexto, se manifestam em problemas que envolvem o suporte da medida a priori. E como veremos esses argumentos só são possíveis de se reproduzir quando a medida a priori tem suporte total. Para não deixar a notação muito carregada, correndo o risco de focar nossa atenção em detalhes irrelevantes, vamos introduzir a notação temporaria $\mathscr{L} \equiv \mathscr{L}_{\log g}$. Começaremos provando que $\left\{\mathscr{L}^{n} \varphi, n \geq 0\right\}$ é uma família equicontínua para qualquer que seja $\varphi$ fixada em $C(\Omega)$. De fato, pela definição do operador de Ruelle temos

$$
\begin{aligned}
\left|\mathscr{L}^{n} \varphi(x)-\mathscr{L}^{n} \varphi(y)\right| & \leq\left|\int_{M^{n}}\left[\exp \left(S_{n} \log g(\mathbf{a} x)\right) \varphi(\mathbf{a} x)-\exp \left(S_{n} \log g(\mathbf{a} y)\right) \varphi(\mathbf{a} y)\right] \prod_{i=1}^{n} d \mu\left(a_{i}\right)\right| \\
& \leq\left|\int_{M^{n}} \prod_{i=0}^{n-1} g\left(\sigma^{i}(\mathbf{a} x)\right)[\varphi(\mathbf{a} x)-\varphi(\mathbf{a} y)] \prod_{i=0}^{n} d \mu\left(a_{i}\right)\right| \\
& +\left|\int_{M^{n}} \varphi(\mathbf{a} y)\left[\prod_{i=0}^{n-1} g\left(\sigma^{i}(\mathbf{a} x)\right)-\prod_{i=0}^{n-1} g\left(\sigma^{i}(\mathbf{a} y)\right)\right] \prod_{i=0}^{n} d \mu\left(a_{i}\right)\right| .
\end{aligned}
$$


Os dois termos do lado direito podem ser estimados por

$$
\begin{aligned}
\sup _{\mathbf{a} \in M^{n}}\{|\varphi(\mathbf{a} x)-\varphi(\mathbf{a} y)|\} \mid \int_{M^{n}} & \prod_{i=0}^{n-1} g\left(\sigma^{i}(\mathbf{a} x)\right) \prod_{i=1}^{n} d \mu\left(a_{i}\right) \mid \\
& +\|\varphi\|_{0} \int_{M^{n}} \prod_{i=0}^{n-1} g\left(\sigma^{i}(\mathbf{a} y)\right)\left|\frac{\prod_{i=0}^{n-1} g\left(\sigma^{i}(\mathbf{a} x)\right)}{\prod_{i=0}^{n-1} g\left(\sigma^{i}(\mathbf{a} y)\right)}-1\right| \prod_{i=1}^{n} d \mu\left(a_{i}\right) .
\end{aligned}
$$

Como $g \in G_{0}(\Omega)$ segue do Teorema de Fubini que a integral iterada no primeiro termo é igual a 1 . O segundo termo pode ser limitado, similarmente, usando-se a definição de $D_{g}^{\star}(x, y)$, o que nos fornece a seguinte desigualdade

$$
\left|\mathscr{L}^{n} \varphi(x)-\mathscr{L}^{n} \varphi(y)\right| \leq \sup _{\mathbf{a} \in M^{n}}\{|\varphi(\mathbf{a} x)-\varphi(\mathbf{a} y)|\}+\|\varphi\|_{0} \cdot D_{g}^{\star}(x, y) .
$$

Como $\varphi$ é uma função contínua e $\log g$ satisfaz a condição fraca de Walters a desigualdade anterior implica que $\left\{\mathscr{L}^{n} \varphi, n \geq 0\right\}$ é uma sequência equicontínua. Lembrando que $g \in G_{0}(\Omega)$ temos pela definição do operador de Ruelle que $\left\|\mathscr{L}^{n} \varphi\right\|_{0} \leq\|\varphi\|_{0}$ para toda $\varphi \in C(\Omega)$ e para todo $n \in \mathbb{N}$. Esta desigualdade implica que o fecho de $\left\{\mathscr{L}^{n} \varphi, n \geq 0\right\}$ na topologia uniforme é uniformemente limitado em $C(\Omega)$. Portanto podemos aplicar o Teorema de ArzelàÀscoli para a família $\left\{\mathscr{L}^{n} \varphi, n \geq 0\right\}$ no intuito de obter uma subsequência $\left(n_{i}\right) \subset \mathbb{N}$ e uma função $\varphi^{\star} \in C(\Omega)$ tais que $\mathscr{L}^{n_{i}} \varphi \longrightarrow \varphi^{\star}$ uniformemente. Começaremos mostrando que $\varphi^{\star}$ é uma função constante. Note que a identidade $\mathscr{L}(\mathbf{1})=1$ implica as seguintes desigualdades $\min (\varphi) \leq \min (\mathscr{L}(\varphi)) \leq \cdots \leq \min \left(\varphi^{\star}\right)$.

Afirmaçãa 1. Para todo $k \in \mathbb{N}$ temos $\min \left(\mathscr{L}^{k} \varphi^{\star}\right)=\min \left(\varphi^{\star}\right)$. De fato, temos que $\min \left(\mathscr{L}^{k} \varphi^{\star}\right)=$ $\min \left(\mathscr{L}^{k}\left(\lim \mathscr{L}^{n_{i}} \varphi\right)\right)=\min \left(\lim \mathscr{L}^{n_{i}+k} \varphi\right)=\lim \left(\min \left(\mathscr{L}^{n_{i}+k} \varphi\right)\right)=\min \left(\varphi^{\star}\right)$, onde a última igualdade segue da monotonicidade da sequencia $\min \mathscr{L}^{k} \varphi$ e de $\min \mathscr{L}^{n_{i}} \varphi \longrightarrow \min \varphi^{\star}$.

Dado $\varepsilon>0$ escolha $x \in \Omega$ e $N \in \mathbb{N}$ tais que $\min \left(\mathscr{L}^{N} \varphi^{\star}\right)=\mathscr{L}^{N} \varphi^{\star}(x)$ e $\left\{\mathbf{a} x, \mathbf{a} \in M^{N}\right\}$ é $\varepsilon$-denso em $\Omega$.

Afirmação 2. Para todo $y \in \sigma^{-N} x$ temos que $\varphi^{\star}(y)=\min \left(\varphi^{\star}\right)$. Pela afirmação 1 e pela escolha de $x$, temos $\mathscr{L}^{N} \varphi^{\star}(x)=\min \left(\varphi^{\star}\right)$. Seja $z \in \Omega$ tal que $\varphi^{\star}(z)=\min \left(\varphi^{\star}\right)$, então

$$
\int_{M^{N}} g(\mathbf{a} x) g(\sigma \mathbf{a} x) \cdots g\left(\sigma^{N-1} \mathbf{a} x\right) \varphi^{\star}(\mathbf{a} x) \prod_{i=1}^{N} d \mu\left(a_{i}\right)=\mathscr{L}^{N} \varphi^{\star}(x)=\min \left(\varphi^{\star}\right)=\varphi^{\star}(z) .
$$

Usando a identidade $\mathbf{1}(x)=\mathscr{L}_{\log g}^{N} \mathbf{1}(x)$, segue da equação acima que

$$
0=\int_{M^{N}} g(\mathbf{a} x) g(\sigma \mathbf{a} x) \cdots g\left(\sigma^{N-1} \mathbf{a} x\right)\left[\varphi^{\star}(\mathbf{a} x)-\varphi^{\star}(z)\right] \prod_{i=1}^{N} d \mu\left(a_{i}\right) .
$$

Usando a continuidade de $\varphi^{\star}$ e a hipótese de que $\operatorname{supp}(\mu)=M$ é fácil ver que $\varphi^{\star}(\mathbf{a} x)=\varphi^{\star}(z)$ para qualquer $\mathbf{a} \in M^{N}$. Como $\varphi^{\star}$ é contínua e constante sobre a fibra $\left\{\mathbf{a} x, \mathbf{a} \in M^{N}\right\}$, que é $\varepsilon$-densa em $\Omega$, segue que $\varphi^{\star}$ é uma função constante. 
Agora vamos mostrar a existência e unicidade de um ponto fixo para $\mathscr{L}^{*} \equiv \mathscr{L}_{\log g}^{*}$. Defina o funcional linear $F: C(\Omega) \rightarrow \mathbb{R}$ pondo $F(\varphi)=\varphi^{\star}$. O funcional $F$ é claramente positivo e satisfaz $F(\mathbf{1})=1$. Então segue do Teorema de Riesz-Markov que existe uma única probabilidade Boreliana $\nu \in \mathcal{M}(\Omega)$ que representa $F$. É fácil ver que $\mathscr{L}^{*} \nu=\nu$. Para unicidade suponha que existe outra medida de probabilidade $\gamma \in \mathcal{M}(\Omega)$ tal que $\mathscr{L}^{*} \gamma=\gamma$. É claro que , $\left(\mathscr{L}^{*}\right)^{n} \gamma=\gamma$ para todo $n \in \mathbb{N}$, sendo assim

$$
\int_{\Omega} \varphi d \gamma=\int_{\Omega} \varphi d\left[\left(\mathscr{L}^{*}\right)^{n} \gamma\right]=\int_{\Omega} \mathscr{L}^{n} \varphi d \gamma=\int_{\Omega} \lim _{n \rightarrow \infty} \mathscr{L}^{n} \varphi d \gamma=\int_{\Omega} \varphi^{\star} d \gamma=\varphi^{\star}=\int_{\Omega} \varphi d \nu
$$

Como $\varphi \in C(\Omega)$ é arbitrário segue que $\gamma=\nu$.

Lema 4.2.2. Seja $f$ um potencial satisfazendo a condição fraca de Walters, então $\forall \varepsilon>0$, existe $N>0$ e $a \in \mathbb{R}$ tais que $\forall x, y \in \Omega, \quad \exists w \in \sigma^{-N} x \cap B_{d_{\Omega}}(y, \varepsilon) \operatorname{com} S_{N} f(w) \geq a$.

Demonstração. Para prova veja [48] página 126.

Lema 4.2.3. Seja $f \in C(\Omega)$ um potencial. Então existe um número real $\lambda>0$ e uma medida Boreliana $\nu \in \mathcal{M}(\Omega)$ tais que $\mathscr{L}_{f}^{*} \nu=\lambda \nu$.

Demonstração. A aplicação $\gamma \mapsto \mathscr{L}_{f}^{*} \gamma /\left(\mathscr{L}_{f}^{*} \gamma\right)(\mathbf{1})$ define uma mapa contínuo de $\mathcal{M}(\Omega)$ em si mesmo. O Teorema do Ponto Fixo de Schauder-Tychonoff garante a existência de um ponto fixo $\nu$ para esta aplicação. Tomando $\lambda=\left(\mathscr{L}_{f}^{*} \nu\right)(\mathbf{1})$ o teorema segue.

Agora estamos prontos para provar o resultado principal deste capítulo, o Teorema de Ruelle para potenciais na classe de Walters.

Teorema 4.2.4. Seja $f$ um potencial satisfazendo a condição fraca de Walters e considere o operador de Ruelle associado $\mathscr{L}_{f}: C(\Omega) \rightarrow C(\Omega)$. Então, existe um número real $\lambda_{f}>0$, uma função contínua $h_{f}$ positiva e uma única probabilidade Boreliana $\nu_{f}$ tal que

i) $\mathscr{L}_{f} h_{f}=\lambda_{f} h_{f}, \mathscr{L}_{f}^{*} \nu_{f}=\lambda_{f} \nu_{f}$

ii) Para qualquer $\varphi \in C(\Omega)$ temos

$$
\left\|\lambda_{f}^{-n} \mathscr{L}_{f}^{n} \varphi-h_{f} \int_{\Omega} \varphi d \nu_{f}\right\|_{0} \longrightarrow 0, \quad \text { quando } n \rightarrow \infty .
$$

Demonstração. A prova será dividida em três passos.

Afirmação 1. Seja $\nu$ e $\lambda$ dados pelo Lema 4.2.3. Então para qualquer $f$ satisfazendo a condição fraca de Walters e $\varepsilon_{0}>0$ o conjunto

$$
\Lambda=\left\{\varphi \in C(\Omega): \varphi \geq 0, \nu(\varphi)=1 \text { e } \varphi(x) \leq \exp \left(C_{f}(x, y)\right) \varphi(y) \text { se } d_{\Omega}(x, y)<\varepsilon_{0}\right\}
$$


é convexo, fechado, limitado e uniformemente equicontínuo. Provemos primeiro que $\Lambda$ é não vazio. De fato, para quaisquer $x, y \in \Omega$ temos que

$$
\begin{aligned}
& \mathscr{L}_{f} \mathbf{1}(x)=\int_{M} e^{f(a x)} d \mu(a)=\int_{M} e^{f(a y)} e^{f(a x)-f(a y)} d \mu(a) \\
& \leq \exp \left(\sup _{a \in M} f(a x)-f(a y)\right) \int_{M} e^{f(a y)} d \mu(a) \leq \exp \left(C_{f}(x, y)\right) \mathscr{L}_{f} \mathbf{1}(y) .
\end{aligned}
$$

O conjunto $\Lambda$ é claramente fechado e convexo. Vamos provar que $\Lambda$ é um conjunto limitado. Sejam $x, y \in \Omega$. Pelo Lema 4.2 .2 dados $\varepsilon>0$ e $a \in \mathbb{R}$ existe $N \in \mathbb{N}$ e $y_{0}=\mathbf{a}_{0} x$, onde $\mathbf{a}_{0}=a_{1} \ldots a_{N}$, tais que $d_{\Omega}\left(y_{0}, y\right)<\varepsilon$ e $S_{N} f\left(y_{0}\right) \geq a$. Dado $\delta_{1}>0$ segue da continuidade de $\varphi$ que podemos escolher $\delta>0$ tal que para qualquer a na bola fechada $B\left[\mathbf{a}_{0}, \delta\right] \subset M^{N}$ temos $S_{N} f(\mathbf{a} x) \geq a-\delta_{1}$. Em particular, podemos escolher $\delta$ tal que $B\left[y_{0}, \delta\right] \subset B[y, \varepsilon]$. Portanto segue da definição do operador de Ruelle e da escolha de $\delta$ que

$$
\begin{aligned}
\mathscr{L}^{N} \varphi(x) & =\int_{M^{N}} e^{S_{N} f(\mathbf{a} x)} \varphi(\mathbf{a} x) \prod_{i=1}^{N} d \mu\left(a_{i}\right) \\
& =\int_{\Omega \backslash B\left[\mathbf{a}_{0}, \delta\right]} e^{S_{N} f(\mathbf{a} x)} \varphi(\mathbf{a} x) \prod_{i=1}^{N} d \mu\left(a_{i}\right)+\int_{B\left[\mathbf{a}_{0}, \delta\right]} e^{S_{N} f(\mathbf{a} x)} \varphi(\mathbf{a} x) \prod_{i=1}^{N} d \mu\left(a_{i}\right) \\
& \geq \int_{B\left[\mathbf{a}_{0}, \delta\right]} e^{S_{N} f(\mathbf{a} x)} \varphi(\mathbf{a} x) \prod_{i=1}^{N} d \mu\left(a_{i}\right) \\
& \geq \mu \times \ldots \times \mu\left(B\left[\mathbf{a}_{0}, \delta\right]\right) e^{a-\delta_{1}} \varphi\left(w_{0}\right)
\end{aligned}
$$

onde $w_{0}$ minimiza a função $a_{1} \cdots a_{N} \mapsto \varphi\left(a_{1} \cdots a_{N} x\right)$ em $B[y, \delta]$. Agora observe que $w_{0} \in$ $B[y, \varepsilon]$, usando a compacidade de $\Omega$ e a definição de $\Lambda$ obtemos para todos $x, y \in \Omega$ a seguinte desigualdade $\varphi(y) \leq C_{1} \cdot e^{\delta_{1}-a} \mathscr{L}^{N} \varphi(x)$. Relembrando que $\mathscr{L}_{f}^{*} \nu=\lambda \nu$ e $\nu(\varphi)=1$, obtemos por integração em ambos os lados da desigualdade anterior que $\varphi(y) \leq C_{1} \cdot e^{C+\delta_{1}-a} \nu\left(\mathscr{L}^{N} \varphi(x)\right)=$ $C_{1} \cdot e^{C+\delta_{1}-a} \lambda^{N}$. Portanto $\Lambda$ é limitado. A equicontinuidade uniforme de $\Lambda$ é provada como em [48, p. 129] mutatis mutandis.

Afirmação 2. O operador $\lambda^{-1} \mathscr{L}_{f}$ mapeia $\Lambda$ em $\Lambda$. Sejam $\varphi \in \Lambda$ e $x, y \in \Omega \operatorname{com} d_{\Omega}(x, y)<\epsilon_{0}$. Então

$$
\begin{aligned}
\frac{1}{\lambda} \mathscr{L} \varphi(x) & =\frac{1}{\lambda} \int_{M} e^{f(a x)} \varphi(a x) d \mu(a) \\
& \leq \frac{1}{\lambda} \int_{M} e^{f(a y)} \varphi(a y) \underbrace{\left(e^{f(a x)-f(a y)} e^{C_{f}(a x, a y)}\right)}_{\leq e^{C_{f}(x, y)}} d \mu(a) \\
& \leq \frac{1}{\lambda} \int_{M} e^{f(a y)} \varphi(a y) e^{C_{f}(x, y)} d \mu(a) \\
& =\frac{1}{\lambda} \mathscr{L}_{f} \varphi(y) e^{C_{f}(x, y)}
\end{aligned}
$$


onde a desigualdade $e^{f(a x)-f(a y)} e^{C_{f}(a x, a y)} \leq e^{C_{f}(x, y)}$ é justificada por se observar que $C_{f}(a x, a y)$ é igual a

$$
\sup _{n \geq 1} \sup _{\mathbf{a} \in M^{n}}\left\{(f(\mathbf{a} a x)-f(\mathbf{a} a y))+(f(\sigma \mathbf{a} x)-f(\sigma \mathbf{a} a y))+\cdots+\left(f\left(\sigma^{n-1} \mathbf{a} a x\right)-f\left(\sigma^{n-1} \mathbf{a} a y\right)\right)\right\} .
$$

Segue dai que $C_{f}(a x, a y)+(f(a x)-f(a y)) \leq C_{f}(x, y)$.

As Afirmações 1 e 2 nos permitem usar Teorema do Ponto Fixo de Schauder-Tychonoff no intuito de obter um ponto fixo $h \in \Lambda$ para o operador $\lambda^{-1} \mathscr{L}_{f}$. Este ponto fixo $h$ satisfaz $\mathscr{L}_{f} h=\lambda h, \nu(h)=1$ e $h(x) \leq e^{C_{f}(x, y)} h(y)$ sempre que $d_{\Omega}(x, y)<\epsilon_{0}$. Vamos mostrar que $h>0$. Suponha que exista algum $x \in \Omega$ tal que $h(x)=0$. Então, para todo $n \in \mathbb{N}$ teremos $\mathscr{L}_{f}^{n} h(x)=\lambda^{n} h(x)=0$, sendo assim $h$ deve ser 0 sobre a fibra $\left\{\sigma^{-n} x, n \in \mathbb{N}\right\}$, que é densa, usando que $\mu$ tem suporte total obtemos que $h \equiv 0$ contradizendo o fato de $\nu(h)=1$.

Afirmação 3. Se $g=e^{f} h /(\lambda h \circ T)$, então $g \in G_{0}(\Omega)$ e $D_{g}^{\star}(x, y) \rightarrow 0$ quando $d_{\Omega}(x, y) \rightarrow 0$. A prova é similar à encontrada em [48, p. 130] mutatis mutandis.

Pelo Teorema 4.2.1) temos que $\mathscr{L}_{\log g}^{n} \varphi \stackrel{\|\cdot\|_{0}}{\longrightarrow} \mu(\varphi)$ para toda $\varphi \in C(\Omega)$, onde $\mu \in \mathcal{M}(\Omega)$ é o ponto fixo de $\mathscr{L}_{\log g}^{*}$ em $\mathcal{M}(\Omega)$. Por outro lado

$$
\frac{1}{\lambda^{n}} \mathscr{L}_{f}^{n} \varphi(x)=h(x)\left(\mathscr{L}_{\log g}^{n}(\varphi / h)(x)\right)
$$

então segue que $1 / \lambda^{n} \mathscr{L}_{f}^{n} \varphi \stackrel{\|\cdot\|_{0}}{\longrightarrow} h \cdot \mu(\varphi / h)$. Vamos mostrar que $\mu(\varphi / h)=\nu(\varphi)$, onde $\nu$ é a automedida dada por 4.2.3). Seja $m \in \mathcal{M}(\Omega)$ definida por $m(\varphi)=\nu(h \varphi)$. Então

$$
m\left(\mathscr{L}_{\log g} \varphi\right)=\nu\left(h \cdot \mathscr{L}_{\log g} \varphi\right)=\frac{1}{\lambda}=\nu\left(\mathscr{L}_{f}(\varphi \cdot h)=m(\varphi)\right.
$$

ou seja, $\mathscr{L}_{\log g}^{*} m=m$. Segue da unicidade do ponto fixo que $\nu(h \varphi)=m(\varphi)=\mu(\varphi)$ o que implica que $\nu(\varphi)=\mu(\varphi / h)$.

\subsection{ADENDO: \\ COndiçÕes Equivalentes À CONDiçÃo De Walters}

Ao longo do texto trabalharemos com três definições distintas de o que significa um potencial estar na classe de Walters, direta ou indiretamente. Como já vimos anteriormente, quando estamos com espaço de estados compactos, estas definições podem não coincidir, abaixo veremos que no contexto de espaço de estados discretos elas coincidem. Antes de prosseguirmos vamos esclarecer algumas notações. Nesta seção $\Omega=\{-1,1\}^{\mathbb{N}}$. Consideraremos em $\Omega$ a métrica $d_{\Omega}$ definida pondo-se $d_{\Omega}(x, y)=1 / 2^{n_{0}}$ onde $n_{0}=\min \left\{n \in \mathbb{N}: x_{i} \neq y_{i}\right\}$. Esta métrica gera a topologia produto e tem a conveniência de que as bolas de raio $1 / 2^{n}$ coincidem com cilindros de comprimento $n$. Consideraremos também a métrica $d_{n}$ em $\Omega$ definida pondo-se $d_{n}(x, y)=\max _{0 \leq i \leq n-1} d_{\Omega}\left(\sigma^{i} x, \sigma^{i} y\right)$, onde $\sigma$ naturalmente é o shift à esquerda em $\Omega$ 
C. W. 1: A seguinte definição está em [48]. Seja $f: \Omega \rightarrow \mathbb{R}$ um potencial contínuo, defina $C_{f}(x, y)$ pondo

$$
C_{f}(x, y)=\sup _{n \geq 1} \sup _{\mathbf{a} \in M^{n}} S_{n} f(\mathbf{a} x)-S_{n} f(\mathbf{a} y) .
$$

Diremos que $f$ satisfaz a condição Walters se $C_{f}(x, y) \rightarrow 0$ quando $d(x, y) \rightarrow 0$.

C. W. 2: A próxima definição é encontrada em [5] Diremos que uma função contínua $f: \Omega \rightarrow \mathbb{R}$ satisfaz a condição de Walters se $\varepsilon>0$ existe $\eta>0$ tal que

$$
\forall n \geq 1, \forall x, y \in \Omega, \quad d_{n}(x, y) \leq \eta \Longrightarrow\left|S_{n} f(x)-S_{n} f(y)\right| \leq \varepsilon .
$$

C. W. 3: Dado um potencial $f: \Omega \rightarrow \mathbb{R}$ contínuo, definimos a $n$-ésima variação de $f$ como

$$
\operatorname{var}_{n}(f)=\sup \left\{|f(x)-f(y)|: x, y \in \Omega, x_{i}=y_{i}, \forall 0 \leq i \leq n-1\right\} .
$$

A próxima definção é dada em [50]. O potencial $f: \Omega \rightarrow \mathbb{R}$ está na classe de Walters quando

$$
\lim _{p \rightarrow \infty} \operatorname{var}_{n+p} S_{n} f=0 .
$$

Prova de C. W. $1 \Rightarrow$ C. W. 2. Dado $\varepsilon>0$ existe $\delta$ tal que $d_{\Omega}\left(x_{1}, y_{1}\right)<\delta$ implica que

$$
C_{f}\left(x_{1}, y_{1}\right)=\sup _{n \geq 1} \sup _{\mathbf{a} \in M^{n}} S_{n} f\left(\mathbf{a} x_{1}\right)-S_{n} f\left(\mathbf{a} y_{1}\right)<\varepsilon .
$$

Em particular, ponhamos $\delta<1 / 2$. Agora seja $\eta \leq \delta$, se $x, y \in \Omega$ são tais que $d_{n}(x, y) \leq \eta$ então temos que $x=\mathbf{a} x_{1}, y=\mathbf{a} y_{1}$ onde $\mathbf{a} \in M^{n}$. Em particular, pela definição de $d_{n}$ temos que $d_{\Omega}\left(x_{1}, y_{1}\right) \leq \eta \leq \delta$, portanto vale (4.5). Assim sendo, conluimos que $\forall x, y \in \Omega, \forall n \geq 1$ com $d_{n}(x, y) \leq \eta, \eta \leq \delta$, vale que $\left|S_{n} f(x)-S_{n} f(y)\right| \leq \varepsilon$.

Prova de C. W. $2 \Rightarrow$ C. W. 3. Comecemos com a seguinte observação, dado $\eta>0$ podemos escolher $p$ é suficientemente grande de tal modo que para todos $x, y \in \Omega$, com $x_{i}=y_{i}$ para $0 \leq i \leq n+p-1$, tenhamos $d_{n}(x, y) \leq \eta$. De fato basta observar que temos a seguinte estimativa,

$$
d_{n}(x, y) \leq d_{\Omega}\left(\mathbf{a} x_{1}, \mathbf{a} y_{1}\right)=\frac{d\left(x_{1}, y_{1}\right)}{2^{p-1}} \leq \frac{M}{2^{p-1}}
$$

onde $M=\max _{x, y \in \Omega} d_{\Omega}(x, y)$. Sendo assim, basta escolher $p$ de modo que $M / 2^{p-1}<\eta$.

Pois bem, dado $\varepsilon>0$ seja $\eta>0$ proveniente da definição de condição C.W.2, então tome $p \in \mathbb{N}$ tal que para todos $x, y \in \Omega \operatorname{com} x_{i}=y_{i}, i=0, \ldots, n+p-1$, tenhamos $d_{n}(x, y) \leq \eta$, daí temos, por hipótese, que $\forall x, y \in \Omega \operatorname{com} x_{i}=y_{i}, 0 \leq i \leq n+p-1,\left|S_{n} f(x)-S_{n} f(y)\right| \leq \varepsilon$. O exposto acima nos fornece que

$$
\operatorname{var}_{n+p}(f)=\sup _{\substack{x, y \in \Omega, x_{i}=y_{i} \\ 0 \leq i \leq n+p-1}}\left|S_{n} f(x)-S_{n} f(y)\right| \leq \varepsilon .
$$

Tomando o sup em $n$ e o limite quando $p \rightarrow \infty$ segue o resultado.

Prova de C. W. $\mathbf{3} \Rightarrow$ C. W. $\mathbf{1}$. A prova deste item reside em observar que tomando por exemplo, $\delta<1 / 2^{p}$ temos que $d_{\Omega}(x, y)<\delta$ implica $x=\mathbf{a} x_{1}, y=\mathbf{a} x_{1}$, com $\mathbf{a} \in M^{p}$. O Resultado segue trivialmente desta observação. 


\section{Capítulo 5}

\section{O OPERADOR DE RUELle E O FORMALISMO DLR}

O objetivo deste capítulo é ver como as medidas de Gibbs obtidas através do formalismo do Operador de Ruelle, isto é, as automedidas do operador dual, se relacionam com as outras noções de medidas de Gibbs provenientes do formalismo da Mecânica Estatística. Abaixo vamos relembrar as noções de medidas de Gibbs no sentido do limite termodinâmico e medidas de Gibbs no sentido do Formalismo DLR. Toda a discussão abaixo é fortemente inspirada em 9]. Assim como nos capítulos anteriores, $M$, nosso espaço de estados, é um espaço métrico onde estamos considerando sobre $M$ a sigma álgebra de Borel. Lembrando que $\mu$ denota uma medida a-priori Boreliana sobre $M$. Como sempre $\Omega=M^{\mathbb{N}}$ munido da topologia produto. Denotaremos por $\mathscr{F}$ a sigma álgebra produto em $\Omega$.

\subsection{Medidas de GibBs Duais}

Inspirados no formalismo de Ruelle somos motivados a definir o conceito de medidas de GibbsDuais.

Definição 5.1.1. Seja $f$ um potencial contínuo. Dizemos que uma probabilidade $\nu \in \mathcal{M}(\Omega)$ é uma medida de Gibbs dual para $f$ se existe um número real $\lambda>0$ tal que $\mathscr{L}_{f}^{*} \nu=\lambda \nu$. Denotamos o conjunto das medidas de Gibbs duais para $f$ por $\mathcal{G}^{*}(f)$.

Nos capítulo anterior foi mostrado no Lema 4.2.3 que $\mathcal{G}^{*}(f)$ é sempre não vazio. Vimos também que se o potencial $f$ está na classe de Walters o conjunto $\mathcal{G}^{*}(f)$ é unitário, neste caso se o potencial estiver normalizado a auto medida é shift invariante. 


\subsection{Medidas De GibBs no SEntido DLR E INTERAÇÕES NO LATICE $\mathbb{N}$}

Seja, $\Phi=\left\{\Phi_{A}\right\}_{A \Subset \mathbb{N}}$ um potencial de interação, ver o apendice A. Lembre que o Hamiltoniano associado a $\Phi$ é dado por

$$
H_{n}(x)=\sum_{A \Subset \mathbb{N},} \Phi_{A \cap \Lambda_{n}=\varnothing}(x) .
$$

Existem casos em que é razoável assumir que o Hamiltoniano acima provém de algum potencial contínuo $f$ ou seja $H_{n}(x)=f(x)+f(\sigma x) \cdots+f\left(\sigma^{n-1} x\right)$, ver por exemplo [6], [39], [48], e assim o faremos. Associado a este Hamiltoniano temos a família $\left(h_{n}\right)_{n \in \mathbb{N}}$ de funções dadas por

$$
h_{n}(x)=\exp \left[-H_{n}(x)\right]
$$

cada $h_{n}$ é chamada de o fator de Boltzman. A proposição abaixo mostra que $\left(h_{n}\right)_{n \in \mathbb{N}}$ é uma pré-modificação, para relembrar a definição veja A.3.6

Proposição 5.2.1. A familia de funções $\left(h_{n}\right)_{n \in \mathbb{N}}$ dadas por (5.1) é uma pré-modificação.

Demonstração. Precisamos mostrar que para quaisquer $x, y \in \Omega, n, r \in \mathbb{N}$, com $\sigma^{n} x=\sigma^{n} y$, que

$$
h_{n}(x) h_{n+r}(y)=h_{n+r}(x) h_{n}(y)
$$

escrevendo explicitamente, precisamos mostrar que

$$
\exp \left[-\left(H_{n}(x)+H_{n+r}(y)\right)\right]=\exp \left[-\left(H_{n+r}(x)+H_{n}(y)\right)\right]
$$

o que é equivalente a mostrar para quaisquer $x, y \in \Omega, n, r \in \mathbb{N}$, com $\sigma^{n} x=\sigma^{n} y$ que

$$
\left.\left.H_{n}(x)+H_{n+r}(y)\right)=H_{n+r}(x)+H_{n}(y)\right] .
$$

Pois bem, note que

$$
H_{n+r}(x)-H_{n}(x)=\sum_{j=n}^{n+r-1} f\left(\sigma^{j} x\right) \underbrace{=}_{\sigma^{n} x=\sigma^{n} y} \sum_{j=n}^{n+r-1} f\left(\sigma^{j} y\right)=H_{n+r}(y)-H_{n}(y)
$$

donde segue o resultado.

Considere a função de partição $Z_{n}$ associada a $h_{n}$ dada por

$$
Z_{n}(x)=\int_{M^{n}} \exp \left[-H_{n}(\mathbf{a} x)\right] d \mu(\mathbf{a}),
$$

é óbvio por continuidade e compacidade que $Z_{n}(x)$ é finito para todo $x \in \Omega$ e todo $n \in \mathbb{N}$. Em outras palavas $f$ é um potencial $\mu$-admissível. Segue então da Proposição (A.3.7) a família de funções $\left(h_{n} / Z_{n}\right)_{n \in \mathbb{N}}$ é uma $\mu$-modificação (ver apêndice A definição A.3.3) ). Isto significa que a família $\left\{K_{n}\right\}_{n \in \mathbb{N}}$ como definida abaixo

$$
K_{n}(F, x)=Z_{n}^{-1}(x) \int_{M^{n}} \exp \left[-H_{n}(\mathbf{a} x)\right] 1_{F}(\mathbf{a} x) d \mu(\mathbf{a})
$$

é uma especificação, a qual chamamos de especificação Gibbssiana. 
Definição 5.2.2 (Medidas de Gibbs no sentido DLR). Seja $\left\{K_{n}\right\}_{n \in \mathbb{N}}$ a especificação Gibbssiana associada ao potencial $f$ construída acima. Definimos o conjunto das medidas de Gibbs no sentido DLR para o potencial $f$, denotado por $\mathcal{G}^{D L R}(f)$, como sendo

$$
\left\{\nu \in \mathcal{M}(\Omega): \nu\left(F \mid \sigma^{n} \mathscr{F}\right)(y)=K_{n}(F, y) \text {, para } y, \nu \text {-qtp } \forall F \in \mathscr{F}, \forall n \in \mathbb{N}\right\}
$$

Observação 5.2.3. Seja $\left\{K_{n}\right\}_{n \in \mathbb{N}}$ a especificação Gibbssiana determinada pelo potencial contínuo $f$. Então, para qualquer função contínua $g: \Omega \rightarrow \mathbb{R}$, e qualquer $z \in \Omega$ fixado, temos que

$$
K_{n+r} \circ K_{n}=K_{n+r} .
$$

Isso decorre das proposiçôes (5.2.1) e (A.3.7) que garante $\left\{K_{n}\right\}_{n \in \mathbb{N}}$ ser uma especificaçao e por conseguinte satisfazer as condições de consistência, veja Definição (A.2.1).

\subsection{Medidas de GibBs no Limite Termodinâmico}

Considere a especificação Gibbssiana $\left\{K_{n}\right\}_{n \in \mathbb{N}}$ associada a um potencial contínuo $f \in C(\Omega)$ como definida em 5.2 . Seja $\left(y_{n}\right)$ uma sequência em $\Omega$ e $\left(K_{n}\left(\cdot, y_{n}\right)\right)$ uma sequência de probabilidades Borelianas em $\Omega$. Considere o conjunto

$$
\mathscr{C}:=\left\{K_{n}\left(\cdot, y_{n}\right): n \in \mathbb{N}, y_{n} \in \Omega\right\} \subset \mathcal{M}(\Omega)
$$

segue da compacidade de $\mathcal{M}(\Omega)$ que $\left(K_{n}\left(\cdot, y_{n}\right)\right)$ tem pelo menos uma subsequência $\left(K_{n_{k}}\left(\cdot, y_{n_{k}}\right)\right)$ tal que $y_{n_{k}} \rightarrow y \in \Omega$ e $\left(K_{n_{k}}\left(\cdot, y_{n_{k}}\right)\right) \rightarrow \nu^{y} \in \mathcal{M}(\Omega)$. Em particular o conjunto dos pontos de acumulação de $\mathscr{C}$ é não vazio.

Definição 5.3.1 (Medidas de Gibbs no Limite Termodinâmico). Definimos o conjunto $\mathcal{G}^{T L}(f)$ como sendo o fecho da envoltória convexa do conjunto dos pontos de acumulação de $\mathscr{C} \mathrm{em}$ $\mathcal{M}(\Omega)$. Um elemento em $\mathcal{G}^{T L}(f)$ é dito uma medida de Gibbs no limite termodinâmico.

\section{$5.4 \mathcal{G}^{*} \subset \mathcal{G}^{T L} \subset \mathcal{G}^{D L R}$}

O Objetivo desta seção é mostrar que a medida de gibbs obtida atravéz do formalismo do operador de Ruelle, no contexto de espaço de estados compactos, via os Teoremas 2.3.4 e 4.2.4 satisfazem as outras noções de medidas de Gibbs, a saber noções já definidas acima de medidas de Gibbs no sentido do limite termodinâmico e medidas de Gibbs no sentido DLR.

$\mathcal{G}^{*}(f) \subset \mathcal{G}^{T L}(f)$

No que segue provamos que a noção medida de Gibbs proveniente do formalismo do operador de Ruelle satisfaz a noção de medida de Gibbs no sentido do limite Termodinâmico

Proposição 5.4.1. Para qualquer potencial $f \in W(\Omega)$ temos que $\mathcal{G}^{*}(f) \subset \mathcal{G}^{T L}(f)$. 
Demonstração. Consideremos a especificação Gibbssiana $\left\{K_{n}\right\}_{n \in \mathbb{N}}$ proveniente de um potencial contínuo $f$,

$$
K_{n}(F, x)=\frac{1}{Z_{n}(x)} \int_{M^{n}} 1_{F}(\mathbf{a} x) \exp \left(-H_{n}(\mathbf{a} x)\right) d \mu(\mathbf{a}),
$$

esta expressão pode ser reescrita em termos do formalismo do operador de Ruelle do seguinte modo

$$
K_{n}(F, x)=\frac{1}{Z_{n}(x)} \int_{M^{n}} 1_{F}(x) \exp \left(-\beta H_{n}(\mathbf{a} x)\right) d \mu(\mathbf{a})=\frac{\mathscr{L}_{f}^{n}\left(1_{F}\right)\left(\sigma^{n}(x)\right)}{\mathscr{L}_{f}^{n}(1)\left(\sigma^{n}(x)\right)} .
$$

escrevendo de outro modo, temos que

$$
K_{n}(\cdot, x)=\frac{1}{\mathscr{L}_{f}^{n}(1)\left(\sigma^{n}(x)\right)}\left[\left(\mathscr{L}_{f}\right)^{*}\right]^{n}\left(\delta_{\sigma^{n}(x)}\right) .
$$

Se $f$ é um potencial na classe de Walters, usando a expressão de $S_{n} f$ em termos de $S_{n} \bar{f}$, ver (2.6), temos

$$
\mathscr{L}_{f}^{n}(1)\left(\sigma^{n}(x)\right)=\lambda^{n} h_{f}\left(\sigma^{n}(x)\right) \mathscr{L}_{\bar{f}}^{n}\left(\frac{1}{h_{f}}\right)\left(\sigma^{n}(x)\right),
$$

onde $\lambda$ é o autovalor, $h_{f}$ a autofunção do operador de Ruelle $\mathscr{L}_{f}$ e $\bar{f}=f+\log h_{f}-\log h_{f} \circ \sigma-\log \lambda$ é o potencial normalizado associado a $f$. Portanto fixada uma função contínua $\varphi$ temos para todo $n \in \mathbb{N}$ que

$$
K_{n}(\varphi, x)=\frac{\mathscr{L}_{f}^{n}(\varphi)\left(\sigma^{n}(x)\right)}{\lambda^{n} h_{f}\left(\sigma^{n}(x)\right) \mathscr{L}_{\bar{f}}^{n}\left(\frac{1}{h_{f}}\right)\left(\sigma^{n}(x)\right)}=\frac{\mathscr{L}_{\bar{f}}^{n}\left(\frac{\varphi}{h_{f}}\right)\left(\sigma^{n}(x)\right)}{\mathscr{L}_{\bar{f}}^{n}\left(\frac{1}{h_{f}}\right)\left(\sigma^{n}(x)\right)}
$$

o que implica para cada função contínua $\varphi \in C(\Omega)$ e cada $x \in \Omega$ fixados, que

$$
\lim _{n \rightarrow \infty} K_{n}(\varphi, x)=\nu_{f}(\varphi)
$$

onde $\nu$ é a automedida de $\mathscr{L}_{f}^{*}$. Provando que $\mathcal{G}^{*}(f) \subset \mathcal{G}^{T L}$.

$\mathcal{G}^{T L} \subset \mathcal{G}^{D L R}$

No que vem provamos que a noção de medida de Gibbs proveniente do formalismo do operador de Ruelle coincide com a noção de medida de Gibbs no sentido DLR.

Teorema 5.4.2. Para qualquer potencial contínuo $f$ temos a inclusão, $\mathcal{G}^{T L}(f) \subset \mathcal{G}^{D L R}(f)$.

Demonstração. Suponha que $K_{n_{k}}\left(\cdot, z_{n_{k}}\right) \rightarrow \mu$, isto é, $\nu \in \mathcal{G}^{T L}(f)$. Como $f$ é um potencial contínuo temos para qualquer função contínua $g: \Omega \rightarrow \mathbb{R}$ que o mapa

$$
y \mapsto K_{n}(g, y)
$$

é contínuo. Usando a definição de convergência fraca, as condições de consistência para $\left\{K_{n}\right\}$ e a continuidade de $g$, respectivamente, temos

$$
\left(\nu K_{n}\right) g=\lim _{k \rightarrow \infty} K_{n_{k}} \circ K_{n} g=\lim _{k \rightarrow \infty} K_{n_{k}} g=\nu(g) .
$$

Mostramos que $\nu$ é um ponto fixo para a especificação $\left\{K_{n}\right\}_{n \in \mathbb{N}}$, invocando a Proposição A.1.2 concluímos que $\nu \in \mathcal{G}^{D L R}(f)$. 


\section{Capítulo 6}

\section{DECAimento DE CORRELAÇÕES E AusÊNCIA DE BuraCo EsPECTRAL}

\subsection{Buraco Espectral e Decaimento de CorrelaÇÕes}

Nesta seç̧ão vamos seguir de perto a referência [2].

Definição 6.1.1. Considere o espaço de probabilidade $(\Omega, \mathcal{F}, \nu)$. Seja $\sigma$ o shift à esquerda em $\Omega$. Para cada $\varphi_{1}$ e $\varphi_{2}$ em $L^{2}(\Omega, \nu)$ definimos a função de correlação $C_{\varphi_{1}, \varphi_{2}, \nu}: \mathbb{Z} \rightarrow \mathbb{R}$ pondo

$$
C_{\varphi_{1}, \varphi_{2}, \nu}(n)=\int_{\Omega}\left(\varphi_{1} \circ \sigma^{n}\right) \varphi_{2} d \nu-\int_{\Omega} \varphi_{1} d \nu \int_{\Omega} \varphi_{2} d \nu
$$

Teorema 6.1.2. Suponha que $f \in W(\Omega)$ seja um potencial para o qual o operador de Ruelle $\mathscr{L}_{f}$ tem a propriedade do buraco espectral. Considere a medida $\mu_{f}=h_{f} \nu_{f}$, onde $\nu_{f}$ é a automedida dada pelo Teorema 4.2.4. Então a função de correlação $C_{\varphi_{1}, \varphi_{2}, \mu_{f}}(n)$ decai exponencialmente rápido. Mais precisamente, existem $0<\widetilde{\tau}<1$ e $C(\widetilde{\tau})>0$ tais que para todos $\varphi_{1}, \varphi_{2} \in W(\Omega)$ a função de correlação satisfaz:

$$
\left|C_{\varphi_{1}, \varphi_{2}, \mu_{f}}(n)\right|=\left|\int_{\Omega}\left(\varphi_{1} \circ \sigma^{n}\right) \varphi_{2} d \mu_{f}-\int_{\Omega} \varphi_{1} d \mu_{f} \int_{\Omega} \varphi_{2} d \mu_{f}\right| \leq C_{1} \widetilde{\tau}^{n} .
$$

onde $C_{1}=C(\widetilde{\tau})\left\|h_{f}\right\|_{0}\left(\int_{\Omega}\left|\varphi_{1}\right| d \nu_{f}\right)\left\|\varphi_{2}\right\|$.

Antes de provar o teorema vamos estabelecer dois lemas auxiliares.

Lema 6.1.3. A projeção espectral $\pi_{f} \equiv \pi_{\mathscr{L}_{f}}$ é dada por $\pi_{f}(\varphi)=\left(\int_{\Omega} \varphi d \nu_{f}\right) \cdot h_{f}$.

Demonstração. Sabemos que os operadores $\pi_{f}$ e $\mathscr{L}_{f}$ comutam. Pelo Teorema de Ruelle 4.2.4 temos que

$\lim _{n \rightarrow \infty}\left(1 / \lambda^{n}\right) \mathscr{L}_{f}^{n} \varphi=h_{f} \int_{\Omega} \varphi d \nu_{f}$ uniformemente. Como $\pi_{f}$ é limitado temos que

$$
\left\|\pi_{f}\left(\lambda^{-n} \mathscr{L}_{f}^{n} \varphi-h_{f} \int_{\Omega} \varphi d \nu_{f}\right)\right\|_{0} \leq\left\|\pi_{f}\right\|\left\|\lambda^{-n} \mathscr{L}_{f}^{n} \varphi-h_{f} \int_{\Omega} \varphi d \nu_{f}\right\|_{0} \rightarrow 0,
$$


quando $n \rightarrow \infty$. Visto que $\left.\pi_{f}\left(\lambda^{-n} \mathscr{L}_{f}^{n} \varphi\right)\right)=\lambda^{-n} \mathscr{L}_{f}^{n} \pi_{f}(\varphi)=\lambda^{n} \lambda^{-n} \pi_{f}(\varphi)=\pi_{f}(\varphi)$ obtemos

$$
\pi_{f}(\varphi)=\pi_{f}\left(h_{f} \int_{\Omega} \varphi d \nu_{f}\right)=\int_{\Omega} \varphi d \nu_{f} \cdot \pi_{f}\left(h_{f}\right)=\int_{\Omega} \varphi d \nu_{f} \cdot h_{f}
$$

o seguinte lema será bastante útil no que segue

Lema 6.1.4. Sejam $\varphi_{1}, \varphi_{2} \in W(\Omega)$ então $\mathscr{L}_{f}^{n}\left(\varphi_{1} \circ \sigma^{n} \cdot \varphi_{2} \cdot h_{f}\right)=\varphi_{1} \mathscr{L}_{f}^{n}\left(\varphi_{2} h_{f}\right)$.

Demonstração. A prova é um cálculo corriqueiro. Seja $x \in \Omega$ e $\varphi \in W(\Omega)$ então temos que $\mathscr{L}_{f}^{n} \varphi(x)=\int_{M^{n}} \varphi(\mathbf{a} x) e^{S_{n}(\mathbf{a} x)} d \mu(\mathbf{a})$. Visto que $\varphi_{1} \circ \sigma^{n}(\mathbf{a} x)=\varphi_{1}(x) \quad \forall \mathbf{a} x, \mathbf{a} \in M^{n}$ e $\forall x \in \Omega$ temos que

$$
\begin{aligned}
\mathscr{L}_{f}^{n}\left(\varphi_{1} \circ \sigma^{n} \varphi_{2} h_{f}\right)(x) & =\int_{M^{n}} \varphi_{1} \circ \sigma^{n} \varphi_{2} h_{f}(\mathbf{a} x) e^{S_{n}(\mathbf{a} x)} d \mu(\mathbf{a}) \\
& =\varphi_{1}(x) \int_{M^{n}}\left(\varphi_{2} h_{f}\right)(\mathbf{a} x) e^{S_{n}(\mathbf{a} x)} d \mu(\mathbf{a}) .
\end{aligned}
$$

Agora a prova do principal teorema desta seção.

Prova do Teorema 6.1.2. Como $\mu_{f}=h_{f} d \nu_{f}$ segue da definição de função de correlação que

$$
\left|C_{\varphi_{1}, \varphi_{2}, \mu_{f}}(n)\right|=\left|\int_{\Omega}\left(\varphi_{1} \circ \sigma^{n}\right) \varphi_{2} h_{f} d \nu_{f}-\int_{\Omega} \varphi_{1} h_{f} d \nu_{f} \int_{\Omega} \varphi_{2} h_{f} d \nu_{f}\right| .
$$

Note que $\left(\mathscr{L}_{f}^{*}\right)^{n} \nu_{f}=\lambda_{f}^{n} \nu_{f}$ portanto o lado direito da igualdade acima é igual a

$$
\left|\int_{\Omega} \lambda_{f}^{-n} \mathscr{L}_{f}^{n}\left(\left(\varphi_{1} \circ \sigma^{n}\right) \varphi_{2} h_{f}\right) d \nu_{f}-\int_{\Omega} \varphi_{1} h_{f} d \nu_{f} \int_{\Omega} \varphi_{2} h_{f} d \nu_{f}\right| .
$$

Usando o Lema 6.1.4 obtemos

$$
\begin{aligned}
\left|C_{\varphi_{1}, \varphi_{2}, \mu_{f}}(n)\right| & =\left|\int_{\Omega} \lambda_{f}^{-n} \mathscr{L}_{f}^{n}\left(\left(\varphi_{1} \circ \sigma^{n}\right) \varphi_{2} h_{f}\right) d \nu_{f}-\int_{\Omega} \varphi_{1} h_{f} d \nu_{f} \int_{\Omega} \varphi_{2} h_{f} d \nu_{f}\right| \\
& =\left|\int_{\Omega} \varphi_{1} \lambda_{f}^{-n} \mathscr{L}_{f}^{n}\left(\varphi_{2} h_{f}\right) d \nu_{f}-\int_{\Omega} \varphi_{1} h_{f} d \nu_{f} \int_{\Omega} \varphi_{2} h_{f} d \nu_{f}\right| \\
& \leq\left|\int_{\Omega} \varphi_{1}\left(\lambda_{f}^{-n} \mathscr{L}_{f}^{n}\left(\varphi_{2} h_{f}\right)-h_{f} \int_{\Omega} \varphi_{2} h_{f} d \nu_{f}\right) d \nu_{f}\right| \\
& \leq\left(\int_{\Omega}\left|\varphi_{1}\right| d \nu_{f}\right)\left\|\lambda_{f}^{-n} \mathscr{L}_{f}^{n}\left(\varphi_{2} h_{f}-h_{f} \int_{\Omega} \varphi_{2} h_{f} d \nu_{f}\right)\right\|_{0}
\end{aligned}
$$

Ou seja,

$$
\left|C_{\varphi_{1}, \varphi_{2}, \mu_{f}}(n)\right| \leq\left(\int_{\Omega}\left|\varphi_{1}\right| d \nu_{f}\right)\left\|\lambda_{f}^{-n} \mathscr{L}_{f}^{n}\left(\varphi_{2} h_{f}-h_{f} \int_{\Omega} \varphi_{2} h_{f} d \nu_{f}\right)\right\|_{0}
$$


Estamos supondo que o espectro de $\mathscr{L}_{f}: W(\Omega) \rightarrow W(\Omega)$ consiste de um autovalor simples $\lambda_{f}>$ 0 e um subconjunto de um disco de raio estritamente menor que $\lambda_{f}$. Ponha $\tau=\sup \{|z| ;|z|<$ 1 and $\left.z \cdot \lambda_{f} \in \sigma\left(\mathscr{L}_{f}\right)\right\}$. A existência do buraco espectral garante que $\tau<1$. Considere $\pi_{f}$ a projeção espectral associada ao autovalor $\lambda_{f}$, então pela Proposição B.3.4 o raio espectral associado ao operador $\mathscr{L}_{f}\left(I-\pi_{f}\right)$ é exatamente $\tau \cdot \lambda_{f}$. Como $\left[\mathscr{L}_{f}, \pi_{f}\right]=0$, temos $\forall n \in \mathbb{N}$ que $\left[\mathscr{L}_{f}\left(I-\pi_{f}\right)\right]^{n}=\mathscr{L}_{f}^{n}\left(I-\pi_{f}\right)$. Segue da fórmula do raio espectral (B.2) que para cada escolha de $\widetilde{\tau}>\tau$ existe $n_{0} \equiv n_{0}(\widetilde{\tau}) \in \mathbb{N}$ tal que para todo $n \geq n_{0}$ temos $\left\|\mathscr{L}_{f}^{n}\left(\varphi-\pi_{f} \varphi\right)\right\| \leq \lambda_{f}^{n} \widetilde{\tau}^{n}\|\varphi\|, \forall \varphi \in$ $W(\Omega)$. Portanto existe uma constante $C(\widetilde{\tau})>0$ tal que para todo $n \geq 1$

$$
\left\|\mathscr{L}_{f}^{n}\left(\varphi-\pi_{f} \varphi\right)\right\| \leq C(\widetilde{\tau}) \lambda_{f}^{n} \widetilde{\tau}^{n}\|\varphi\| \quad \forall \varphi \in W(\Omega) .
$$

Usando o Lema 6.1.3 e a conta superior acima na estimativa (6.4) obtemos

$$
\left|C_{\varphi_{1}, \varphi_{2}, \mu_{f}}(n)\right| \leq\left(\int_{\Omega}\left|\varphi_{1}\right| d \nu_{f}\right) C \widetilde{\tau}^{n}\left\|\varphi_{2} h_{f}\right\| \leq C(\widetilde{\tau})\left\|h_{f}\right\|_{0}\left(\int_{\Omega}\left|\varphi_{1}\right| d \nu_{f}\right)\left\|\varphi_{2}\right\| \cdot \tilde{\tau}^{n} .
$$

Obs.: A seguir faremos algumas observações a respeito de duas afirmações que foram feitas na demonstração acima sem a devida explicação. A saber foram as seguintes, 1. o raio espectral associado ao operador $\mathscr{L}_{f}\left(I-\pi_{f}\right)$ é exatamente $\tau \cdot \lambda_{f}$ e a outra $2 .\left[\mathscr{L}_{f}\left(I-\pi_{f}\right)\right]^{n}=\mathscr{L}_{f}^{n}\left(I-\pi_{f}\right)$. Prova de 1. Para a primeira é suficiente observar que todo ponto do espectro de $\mathscr{L}_{f}\left(I-\pi_{f}\right)$ pode ser obtido através de $\lambda_{f}$ aplicando-se uma rotação e uma homotetia, ou seja, através da multiplicação de $\lambda_{f}$ por um número complexo $z$ adequado.

Prova de 2. Para a segunda, usaremos o fato de $\pi_{f}$ e $\mathscr{L}_{f}$ comutarem,

$$
\begin{aligned}
{\left[\mathscr{L}_{f}\left(I-\pi_{f}\right)\right]^{n} } & =\left[\mathscr{L}_{f}-\mathscr{L}_{f} \pi_{f}\right]^{n}=\left[\mathscr{L}_{f}-\pi_{f} \mathscr{L}_{f}\right]^{n} \\
& =\left[\left(I-\pi_{f}\right) \mathscr{L}_{f}\right]^{n}=\left(I-\pi_{f}\right)^{n} \mathscr{L}_{f}^{n} \\
& =\left(I-\pi_{f}\right) \mathscr{L}_{f}^{n}=\mathscr{L}_{f}^{n}-\pi_{f} \mathscr{L}_{f}^{n} \\
& =\mathscr{L}_{f}^{n}-\mathscr{L}_{f}^{n} \pi_{f}=\mathscr{L}_{f}^{n}\left(I-\pi_{f}\right) .
\end{aligned}
$$

\subsection{Ausência de Buraco Espectral no ESPAÇO DE WALTERS}

Nesta seç̧ão vamos apresentar o modelo de Ising de longo alcance no lattice $\mathbb{N}$ no contexto do formalismo termodinâmico. O objetivo é exibir explicitamente um potencial no espaço de Walters para o qual o operador de Ruelle não tenha a propriedade do gap espectral. Ao longo desta secção vamos assumir que o espaço métrico $(M, d)$ é dado por $(\{-1,1\},|\cdot|)$, onde $|\cdot|$ é a função valor absoluto e a probabilidade a priori é a Bernoulli $\nu=(1 / 2)\left[\delta_{\{-1\}}+\delta_{\{1\}}\right]$. 
Exemplo 1. Fixe $\alpha>1$ e considere o potencial $f: \Omega \rightarrow \mathbb{R}$ dado por

$$
f(x)=-\sum_{n \geq 2} \frac{x_{1} x_{n}}{(n-1)^{\alpha}} .
$$

Este potencial não é $\gamma$-Hölder continuo para nenhum $0<\gamma \leq 1$, veja [9].

Por outro lado, quando $\alpha>2$ o potencial $f$ está na classe de Walters. De fato, para qualquer escolha de $n, p \in \mathbb{N}$ temos que $\operatorname{var}_{n+p}\left(f(x)+f(\sigma(x))+\ldots+f\left(\sigma^{n-1}(x)\right)\right)=(n+p)^{-\alpha+1}+(n+$ $p-1)^{-\alpha+1}+\ldots+p^{-\alpha+1}$ o que implica que

$$
\sup _{n \in \mathbb{N}}\left[\operatorname{var}_{n+p}\left(f(x)+f(\sigma(x))+\ldots+f\left(\sigma^{n-1}(x)\right)\right)\right] \sim \sum_{j=p}^{\infty} j^{-\alpha+1} \sim p^{-\alpha+2},
$$

logo $f$ satisfaz a condição de Walters (para lembrar definição de condição de Walters veja o Capítulo 4).

Exemplo 2. Fixe $\alpha>1$ e considere o potencial $f: \Omega \rightarrow \mathbb{R}$ dado por

$$
f(x)=x_{1}+2^{-\alpha} x_{2}+3^{-\alpha} x_{3}+\cdots+n^{-\alpha} x_{n}+\cdots .
$$

É mostrado em [10] que para $3 / 2<\alpha \leq 2$ o potencial $f$ não pertence à classe de Walters e que para $\alpha>2$ temos que $f$ esta na classe de Walters. Essa família de potenciais, é bastante interessante pois para certos valores de $\alpha$ é exibida uma descrição bastante concreta das autofunções do operador de Ruelle associado. Para valores $3 / 2<\alpha \leq 2$ as autofunções obtidas são descontínuas. Para detalhes veja [10].

\section{DeCAimento SUPERPolinomial DE CORRELAÇÕES E AusênCIA Do Buraco EsPeCtral}

A partir de agora fixe $\alpha>1$ e considere o potencial $f: \Omega \rightarrow \mathbb{R}$ dado por

$$
f(x)=-\sum_{n \geq 2} \frac{x_{1} x_{n}}{(n-1)^{\alpha}} .
$$

do Exemplo 1. Quando $1<\alpha<2$, Dyson [14] provou que este modelo tem magnetização espontânea para temperaturas suficientemente baixas. Este fato para estes modelos implica a não unicidade das medidas DLR-Gibbs nestas temperaturas e além disso a pressão não pode ser Fréchet-diferenciável em um espaço de Banach adequado. Para $\alpha=2$ este resultado de transição de fase foi provado por Frölich e Spencer [18].

Como vimos no Exemplo 1, quando $\alpha>2$ o potencial $f$ está na classe de Walters. Por este motivo, no que segue vamos assumir que $\alpha>2$. Neste caso mencionado na introdução o potencial $f$ pertence a um subespaço de $C(\Omega)$ de dimensão infinita, como definido em [8] onde a pressão é Fréchet-analítica. Note que os cálculos anteriores mostram que este espaço não pode estar contido em nenhum espaço de Hölder. Dentro do contexto da mecânica estatística o 
potencial $f$ é normalmente substituído/construído por/através de uma interação absolutamente somável $\Phi=\left(\Phi_{A}\right)_{A \in \mathbb{N}}$, dada por

$$
\Phi_{A}(x)= \begin{cases}\frac{x_{n} x_{m}}{|n-m|^{\alpha}}, & \text { se } A=\{n, m\} \subset \mathbb{N} \text { e } m \neq n ; \\ 0, & \text { caso contrário. }\end{cases}
$$

onde, a notação $A \Subset \mathbb{N}$ é usada para dizer que $A$ é um subconjunto finito ou vazio de $\mathbb{N}$. A relação entre o potencial $f$ e a interação $\Phi$ é descrita de maneira detalhada em [9] e expressada através da seguinte igualdade

$$
H_{n}(x)=\sum_{\substack{A \in \mathbb{N} \\ A \cap \Lambda_{n} \neq \emptyset}} \Phi_{A}(x)=f(x)+f(\sigma x)+\ldots+f\left(\sigma^{n-1} x\right) .
$$

Abaixo, seguindo [9, 24, 40] construiremos uma medida de Gibbs no sentido DLR, ou medida Gibbs-DLR para ser sucinto.

Fixe um potencial $f$, e uma condição de bordo, onde por conveniência vamos escolher como sendo $y=(1,1, \ldots) \in \Omega$. Agora, tome qualquer ponto de acumulação (com respeito a topologia fraca) da sequência $\left(\nu_{n}^{y}\right)_{n \in \mathbb{N}}$ em $\mathscr{P}(\Omega, \mathcal{F})$, onde $\nu_{n}^{y}: \mathcal{F} \rightarrow[0,1]$ é a medida de probabilidade definida para cada $F \in \mathcal{F}$ pela seguinte expressão:

$$
\nu_{n}^{y}(F)=\frac{1}{Z_{n}^{y}} \sum_{\substack{x \in \Omega ; \\ \sigma^{n}(x)=\sigma^{n}(y)}} 1_{F}(x) \exp \left(H_{n}(x)\right), \quad \text { onde } \quad Z_{n}^{y}=\sum_{\substack{x \in \Omega ; \\ \sigma^{n}(x)=\sigma^{n}(y)}} \exp \left(H_{n}(x)\right) .
$$

Quando $\alpha>2$ é um resultado bem conhecido que a sequência $\left(\nu_{n}^{y}\right)_{n \in \mathbb{N}}$ tem um único ponto de acumulação, o qual será denotado por $\nu_{\mathbb{N}}$. Uma prova deste fato, famoso diga-se de passagem, com um ferramental dos sistemas dinâmicos, usando uma consequência do Teorema de Unicidade de Dobrushin juntamente com o formalismo do operador de Ruelle é apresentada em [9. Nosso próximo passo é construir uma medida de probabilidade $\nu_{\mathbb{Z}}$ sobre o espaço simbólico $\hat{\Omega} \equiv\{-1,1\}^{\mathbb{Z}} \equiv\{-1,1\}^{\mathbb{Z n}(-\infty, 0]} \times\{-1,1\}^{\mathbb{N}}$ de tal maneira que

$$
\nu_{\mathbb{N}}(F)=\nu_{\mathbb{Z}}\left(\{-1,1\}^{\mathbb{Z n}(-\infty, 0]} \times F\right), \quad \forall F \in \mathcal{F} .
$$

Fixemos as seguintes notações, $\operatorname{Diag}(\mathbb{Z} \times \mathbb{Z}) \equiv\{(r, r): r \in \mathbb{Z}\}$ e $\mathbb{M} \equiv \mathbb{Z} \times \mathbb{Z} \backslash \operatorname{Diag}(\mathbb{Z} \times \mathbb{Z})$. Defina o subespaço vetorial $\mathbb{J} \subset \mathbb{R}^{\mathbb{M}} \equiv\left\{J_{i j} \in \mathbb{R}:(i, j) \in \mathbb{M}\right\}$ com sendo o conjunto dos pontos em $\mathbb{R}^{\mathbb{M}}$ satisfazendo $\sup _{i \in \mathbb{Z}} \sum_{j \in \mathbb{Z}: j \neq i}\left|J_{i j}\right|<\infty$. Sejam $J_{\mathbb{Z}}$ e $J_{\mathbb{N}}$ dois elementos de $\mathbb{J}$ definidos por $\left(J_{\mathbb{Z}}\right)_{i j}=|i-j|^{-\alpha}$ para todos $(i, j) \in \mathbb{M}$ e $\left(J_{\mathbb{N}}\right)_{i j}=\left(J_{\mathbb{Z}}\right)_{i j}$ se $i, j \in \mathbb{N}$ com $i \neq j$ e $\left(J_{\mathbb{N}}\right)_{i j}=0$ caso contrário. Para cada $n \in \mathbb{N}$ e $J \in \mathbb{J}$ definimos a função $\mathscr{H}_{n}: \hat{\Omega} \times \mathbb{J} \rightarrow \mathbb{R}$ pondo

$$
\mathscr{H}_{n}(z, J)=\sum_{i=-n}^{n} \sum_{j \in \mathbb{Z}: j \neq i} J_{i j} z_{i} z_{j} .
$$

Para qualquer $J \in \mathbb{J}$ fixado e $\hat{y}=(\ldots, 1,1,1, \ldots) \in \hat{\Omega}$ definimos, de modo similar ao anterior, uma medida de probabilidade $\nu_{n}^{\hat{y}, J}$ tal que para cada Boreliano $\hat{F}$ de $\hat{\Omega}$ temos

$$
\nu_{n}^{\hat{y}, J}(\hat{F})=\frac{1}{Z_{n}^{\hat{y}, J}} \sum_{\substack{z \in \hat{\Omega} ; z_{i}=1 \\ \forall i \in \mathbb{Z} \backslash\{-n, \ldots, n\}}} 1_{\hat{F}}(z) \exp \left(\mathscr{H}_{n}(z, J)\right), \quad \text { onde } \quad Z_{n}^{\hat{y}, J}=\sum_{\substack{z \in \hat{\Omega} ; z_{i}=1 \\ \forall i \in \mathbb{Z} \backslash\{-n, \ldots, n\}}} \exp \left(\mathscr{H}_{n}(z, J)\right) .
$$

Para cada $n \in \mathbb{N}$ e $z \in \hat{\Omega}$ fixados, temos as seguintes identidades: 
1. $\mathscr{H}_{n}\left(z, J_{\mathbb{N}}\right)=H_{n}\left(z_{1}, z_{2}, \ldots\right)$;

2. $Z_{n}^{\hat{y}, J_{\mathbb{N}}}=2^{n+1} Z_{n}^{y}$

3. $1_{\{\{-1,1\}} \mathbb{Z n \cap ( - \infty , 0 ] \times F \}}(z)=1_{F}\left(z_{1}, z_{2}, \ldots\right)$.

vamos abrir um parenteses para provar as identidades 1 e 2 , a identidade 3 é evidente.

Prova de 1: Como $\left(J_{\mathbb{N}}\right)_{i j}=0$ para $i<0$ ou $j<0$ temos que

$$
\begin{aligned}
\mathscr{H}_{n}\left(z, J_{\mathbb{N}}\right) & =\sum_{i=-n}^{n} \sum_{j \in \mathbb{Z}: j \neq i} J_{i j} z_{i} z_{j}=\sum_{i=1}^{n} \sum_{j \in \mathbb{N}: j \neq i} J_{i j} z_{i} z_{j} \\
& =\sum_{A \in \mathbb{N}, A \cap \Lambda_{n} \neq \varnothing} \Phi_{A}\left(z_{1}, z_{2}, \ldots\right)=H_{n}\left(z_{1}, z_{2}, \ldots\right) .
\end{aligned}
$$

Prova de 2: Pela identidade 1 temos

$$
Z_{n}^{\hat{y}, J_{\mathbb{N}}}=\sum_{\substack{z \in \hat{\Omega} ; z_{i}=1 \\ \forall i \in \mathbb{Z} \backslash\{-n, \ldots, n\}}} \exp \left(\mathscr{H}_{n}\left(z, J_{\mathbb{N}}\right)\right)=\sum_{\substack{z \in \hat{\Omega} ; z_{i}=1 \\ \forall i \in \mathbb{Z} \backslash\{-n, \ldots, n\}}} \exp \left(H_{n}\left(z_{1}, z_{2}, \ldots\right)\right)
$$

como o hamiltoniano $\mathscr{H}_{n}\left(z, J_{\mathbb{N}}\right)$ não depende das coordenadas $z_{-n}, \ldots, z_{-1}, z_{0}$, e temos $2^{n+1}$ escolhas de strings $z_{-n} \cdots z_{-1} \cdot z_{0}$ segue o resultado. Usando as três identidades acima temos de modo imediato que

$$
\begin{aligned}
\nu_{n}^{\hat{y}, J_{\mathbb{N}}}\left(\left\{\{-1,1\}^{\mathbb{Z} \cap(-\infty, 0]} \times F\right)\right. & =\frac{1}{2^{n+1} Z_{n}^{y}} \sum_{\substack{z \in \hat{\Omega} ; z_{i}=1 \\
\forall i \in \mathbb{Z} \backslash\{-n, \ldots, n\}}} 1_{F}\left(z_{1}, z_{2}, \ldots\right) \exp \left(-H_{n}\left(z_{1}, z_{2}, \ldots\right)\right. \\
& =\nu_{n}^{y}(F)
\end{aligned}
$$

onde na identidade acima usamos novamente que o somando $1_{F}\left(z_{1}, z_{2}, \ldots\right) \exp \left(-H_{n}\left(z_{1}, z_{2}, \ldots\right)\right.$ não depende das coordenadas $z_{-n}, \ldots, z_{-1}, z_{0}$, e temos $2^{n+1}$ escolhas de strings $z_{-n} \cdots z_{-1} \cdot z_{0}$.

Relembrando que $\alpha>2$, segue de resultados clássicos sobre medidas de Gibbs-DLR que a sequência $\left(\nu_{n}^{\hat{y}, J_{\mathbb{N}}}\right)_{n \in \mathbb{N}}$ tem um único ponto de acumulação, o qual chamaremos de $\nu_{\mathbb{Z}}$. Da igualdade anterior é imediato que (6.6) é válido. De acordo com a definição de $\nu_{n}^{\hat{y}, J}$, para qualquer conjunto mensurável $\hat{F}$ e qualquer $n \in \mathbb{N}$ fixados a função $\mathbb{J} \ni J \mapsto \nu_{n}^{\hat{y}, J}(\hat{F})$ é Fréchetanalitica, visto que é a soma finita de funções analíticas. É fácil verificar que para cada par $(i, j) \in \mathbb{M}$ fixado temos

$$
\frac{\partial}{\partial J_{i j}} Z_{n}^{\hat{y}, J}=\sum_{\substack{z \in \hat{\Omega} ; z_{i}=1 \\ \forall i \in \mathbb{Z} \backslash\{-n, \ldots, n\}}} \exp \left(\mathscr{H}_{n}(z, J)\right) \cdot \frac{\partial}{\partial J_{i j}}\left(\mathscr{H}_{n}(z, J)\right)=\sum_{\substack{z \in \hat{\Omega} ; z_{i}=1 \\ \forall i \in \mathbb{Z} \backslash\{-n, \ldots, n\}}} \exp \left(\mathscr{H}_{n}(z, J)\right) \cdot z_{i} z_{j} .
$$

Multiplicando e dividindo o lado direito da expressão acima por $Z_{n}^{\hat{y}, J}$ e usando a definição da integral de Lebesgue obtemos

$$
\frac{\partial}{\partial J_{i j}} Z_{n}^{\hat{y}, J}=Z_{n}^{\hat{y}, J} \int_{\hat{\Omega}} z_{i} z_{j} d \nu_{n}^{\hat{y}, J}(z)
$$


Através de cálculos similares, usando a regra da cadeia, obtemos, para qualquer função mensurável $\psi: \hat{\Omega} \rightarrow \mathbb{R}$, que

$$
\begin{aligned}
\frac{\partial}{\partial J_{i j}} \nu_{n}^{\hat{y}, J}(\psi) & =\frac{\partial}{\partial J_{i j}}\left[\frac{1}{Z_{n}^{\hat{y}, J}} \sum_{\substack{z \in \hat{\Omega} ; z_{i}=1 \\
\forall i \in \mathbb{Z} \backslash\{-n, \ldots, n\}}} \psi(z) \exp \left(\mathscr{H}_{n}(z, J)\right)\right] \\
& =\int_{\hat{\Omega}} \psi(z) z_{i} z_{j} d \nu_{n}^{\hat{y}, J}(z)-\int_{\hat{\Omega}} \psi(z) d \nu_{n}^{\hat{y}, J}(z) \int_{\hat{\Omega}} z_{i} z_{j} d \nu_{n}^{\hat{y}, J}(z) .
\end{aligned}
$$

Antes de prosseguirmos vamos enunciar a desigualdade GKS-II, entretanto, somente na generalidade requerida nesta secção. Para contextos mais gerais ver [15, 26, 28, 30].

Teorema 6.2.1 (Desigualdade GKS-II [28, 30]). Fixe $n \in \mathbb{N}$ e $\left\{n_{1}, n_{2}, \ldots, n_{k}\right\}$ um subconjunto arbitrário de $\{-n, \ldots, n\}$. Se $J \in \mathbb{J}$ satisfaz $J_{i j} \geqslant 0$ para todos $(i, j) \in \mathbb{M}$, então

$$
\int_{\hat{\Omega}} z_{n_{1}} \cdot \ldots \cdot z_{n_{k}} \cdot z_{i} \cdot z_{j} d \nu_{n}^{\hat{y}, J}(z)-\int_{\hat{\Omega}} z_{n_{1}} \cdot \ldots \cdot z_{n_{k}} d \nu_{n}^{\hat{y}, J}(z) \int_{\hat{\Omega}} z_{i} \cdot z_{j} d \nu_{n}^{\hat{y}, J}(z) \geqslant 0
$$

onde $\nu_{n}^{\hat{y}, J}$ denota a medida de probabilidade definida em 6.8.

De agora em diante usaremos $\varphi$ para denotar a função $\varphi(z)=z_{1}$. Estritamente falando $\varphi$ é definida em $\hat{\Omega}$ mas vamos por abuso de notação usar $\varphi(x)$ também para denotar a projeção na primeira coordenada de um elemento de $\Omega$. Se $\widetilde{J} \in \mathbb{J}$ é tal que $\widetilde{J}_{i j} \geqslant 0$ para todos $(i, j) \in \mathbb{M}$ segue de 6.13 e da desigualdade GKS-II que

$$
\left.\frac{\partial}{\partial J_{i j}} \nu_{n}^{\hat{y}, J}(\varphi)\right|_{J=\widetilde{J}} \geqslant 0 .
$$

Esta desigualdade implica que o mapa $\mathbb{J} \ni J \mapsto \nu_{n}^{\hat{y}, J}(\varphi)$ é não decrescente em cada coordenada em $\mathbb{I} \cap[0,+\infty)^{\mathbb{M}}$. Esta monotonicidade, juntamente com as desigualdades $\left(J_{\mathbb{N}}\right)_{i j} \leqslant\left(J_{\mathbb{Z}}\right)_{i j}$ imediatamente implicam que

$$
\int_{\hat{\Omega}} \varphi(z) d \nu_{n}^{\hat{y}, J_{\mathbb{N}}}(z) \leqslant \int_{\hat{\Omega}} \varphi(z) d \nu_{n}^{\hat{y}, J_{\mathbb{Z}}}(z) .
$$

Como $\varphi$ é uma função simples tomando apenas valores -1 e 1 o lado esquerdo da expressão acima se torna, usando a definição da integral de Lebesgue e pela identidade (6.12), igual a

$$
\begin{aligned}
\int_{\hat{\Omega}} \varphi(z) d \nu_{n}^{\hat{y}, J_{\mathbb{N}}}(z) & =\nu_{n}^{\hat{y}, J_{\mathbb{N}}}\left(\left\{z \in \hat{\Omega}: z_{1}=1\right\}\right)-\nu_{n}^{\hat{y}, J_{\mathbb{N}}}\left(z \in \hat{\Omega}: z_{1}=-1\right) \\
& =\nu_{n}^{y}\left(\left\{x \in \Omega: x_{1}=1\right\}\right)-\nu_{n}^{y}\left(x \in \Omega: x_{1}=-1\right) \\
& =\int_{\Omega} \varphi(x) d \nu_{n}^{y}(x) .
\end{aligned}
$$

Substituindo esta última expressão na desigualdade acima obtemos a seguinte desigualdade

$$
\int_{\Omega} \varphi(x) d \nu_{n}^{y}(x) \leqslant \int_{\hat{\Omega}} \varphi(z) d \nu_{n}^{\hat{y}, J_{\mathbb{Z}}}(z) .
$$


Pode ser provado que $\nu_{n}^{\hat{y}, J^{\mathbb{Z}}} \rightarrow \nu$ e que $\nu_{n}^{y} \rightarrow \nu_{\mathbb{N}}$, ver [15]. Observamos ainda que $\nu$ é diferente de $\nu_{\mathbb{Z}}$, definida acima. Então, usando a desigualdade acima, obtemos da definição de convergência fraca que

$$
\int_{\Omega} \varphi(x) d \nu_{\mathbb{N}}(x) \leqslant \int_{\hat{\Omega}} \varphi(z) d \nu(z) .
$$

Vamos provar que o lado esquerdo da desigualdade acima é não negativo, para isso vamos usar a desigualdade GKS-I. Novamente, alertamos que vamos enunciar o resultado apenas na generalidade de que necessitamos. Uma formulação consideravelmente mais geral pode ser encontrada em [15, 26, 27].

Teorema 6.2.2 (GKS-I Inequality [27]). Fixe um número natural $n \geq 1$ um subconjunto $\left\{n_{1}, n_{2}, \ldots, n_{k}\right\} \subset\{-n, \ldots, n\}$ e $J \in \mathbb{J}$ satisfazendo $J_{i j} \geqslant 0$ para todos $(i, j) \in \mathbb{M}$. Seja $\nu_{n}^{\hat{y}, J} a$ medida de probabilidade definida em (6.8), então

$$
\int_{\hat{\Omega}} z_{n_{1}} \cdot \ldots \cdot z_{n_{k}} \cdot z_{i} \cdot z_{j} d \nu_{n}^{\hat{y}, J}(z) \geqslant 0 .
$$

Aplicando a desigualdade GKS-I no lado esquerdo de (6.14) obtemos que

$$
0 \leq \int_{\hat{\Omega}} \varphi(z) d \nu_{n}^{\hat{y}, J_{\mathbb{N}}}(z) \leq \int_{\Omega} \varphi(x) d \nu_{n}^{y}(x) .
$$

Tomando o limite fraco, quando $n \rightarrow \infty$, sa segunda integral acima e usando (6.16) segue que

$$
0 \leqslant \int_{\Omega} \varphi(x) d \nu_{\mathbb{N}}(x) \leqslant \int_{\hat{\Omega}} \varphi(z) d \nu(z) .
$$

Com $\alpha>2$, existe um teorema garantindo que $\int_{\hat{\Omega}} \varphi d \nu(z)=0$, veja [15]. Provamos portanto que

$$
\int_{\Omega} \varphi(x) d \nu_{\mathbb{N}}(x)=0
$$

Para obter a conta inferior na qual estamos interessados, considere para cada $n \geq 1$ o elemento $J^{\{1, n+1\}} \in \mathbb{J}$, dado por $\left(J^{\{1, n+1\}}\right)_{i j}=n^{-\alpha}$ se $(i, j)=(1, n+1)$ e $\left(J^{\{1, n+1\}}\right)_{i j}=0$ caso contrário. De maneira similar como procedemos anteriormente, obtemos usando a desigualdade GKS-II novamente, a monotonicidade, coordenada a coordenada do mapa $\mathbb{J} \ni J \mapsto \nu_{m}^{\hat{y}, J}\left(z_{n+1} z_{1}\right)$, sempre que $m>n$, portanto concluímos que

$$
\int_{\hat{\Omega}} z_{n+1} z_{1} d \nu_{m}^{\hat{y}, J^{\{1, n+1\}}}(z) \leqslant \int_{\hat{\Omega}} z_{n+1} z_{1} d \nu_{m}^{\hat{y}, J_{\mathbb{N}}}(z) .
$$

Note que o lado esquerdo acima pode ser calculado explicitamente como segue (e seu valor independe de $m$ )

$$
\begin{aligned}
\int_{\hat{\Omega}} z_{n+1} z_{1} d \nu_{m}^{\hat{y}, J^{\{1, n+1\}}}(z) & =\sum_{z_{n+1}= \pm 1} \sum_{z_{1}= \pm 1} z_{n+1} z_{1} \exp \left(\frac{z_{n+1} z_{1}}{n^{\alpha}}\right)\left[\sum_{z_{n+1}= \pm 1} \sum_{z_{1}= \pm 1} \exp \left(\frac{z_{n+1} z_{1}}{n^{\alpha}}\right)\right]^{-1} \\
& =\frac{2 \exp \left(n^{-\alpha}\right)-2 \exp \left(n^{-\alpha}\right)}{2 \exp \left(n^{-\alpha}\right)+2 \exp \left(n^{-\alpha}\right)} \\
& =\tanh \left(n^{-\alpha}\right)
\end{aligned}
$$


Por outro lado, usando a igualdade anterior e 6.12 temos para qualquer $m>n$ que

$$
\tanh \left(n^{-\alpha}\right) \leqslant \int_{\hat{\Omega}} z_{n+1} z_{1} d \nu_{m}^{\hat{y}, J_{\mathbb{N}}}(z)=\int_{\Omega} x_{n+1} x_{1} d \nu_{m}^{y}(x)=\int_{\Omega}\left(\varphi \circ \sigma^{n}\right) \varphi d \nu_{m}^{y} .
$$

Considerando a expansão de Taylor da tangente hiperbólica e tomando o limite fraco quando $m \rightarrow \infty$, obtemos para alguma constante $C>0$ a seguinte desigualdade

$$
\int_{\Omega}\left(\varphi \circ \sigma^{n}\right) \varphi d \nu_{\mathbb{N}} \geqslant \tanh \left(n^{-\alpha}\right) \geqslant \frac{C}{|n|^{\alpha}} .
$$

Juntando a desigualdade anterior junto com (6.17) obtemos finalmente que

$$
\frac{C}{|n|^{\alpha}} \leqslant \int_{\Omega}\left(\varphi \circ \sigma^{n}\right) \varphi d \nu_{\mathbb{N}}-\int_{\Omega} \varphi d \nu_{\mathbb{N}} \int_{\Omega} \varphi d \nu_{\mathbb{N}}=C_{\varphi, \varphi, \nu_{\mathbb{N}}}(n) .
$$

Foi mostrado em [9] que $d \mu_{f} \equiv h_{f} d \nu_{f}$, onde $\nu_{f}$ e $h_{f}$ são dados pelo Teorema 4.2.4, estão em to $\mathcal{G}^{D L R}(f)$. Os autores também mostram que para $\alpha>2$ o conjunto $\mathcal{G}^{D L R}(f)$ é unitário e portanto $\mu_{f}=\nu_{\mathbb{N}}$. Este fato juntamente com a continuidade de $h_{f}$ e as desigualdades anteriores mostram que $C_{\varphi, \varphi, \mu_{f}}(n)$ não tem decaimento exponencial. Como $\varphi(x)=x_{1}$ está na classe de Walters segue do Teorema 6.1.2 que $\mathscr{L}_{f}$ não tem a propriedade do buraco espectral. 


\section{Apêndice A}

\section{O Formalismo DLR}

\section{A.1 Preliminares}

1. Seja $S$ um conjunto enumerável infinito e $(E, \mathscr{E})$ um espaço mensurável qualquer. Nossos exemplos favoritos serão $S=\mathbb{Z}^{d}$ e $E=\mathbb{R}$.

2. Uma família $\left(\sigma_{i}\right)_{i \in S}$ de variáveis aleatórias definidas em algum espaço de probabilidade $(\Omega, \mathscr{F}, \mu)$ tomando valores em $(E, \mathscr{E})$ é chamada um campo aleatório. O conjunto $S$ é chamado de conjunto de parâmetros e o espaço mensurável $(E, \mathscr{E})$ é chamado o o espaço de estados do campo aleatório.

3. De acordo com um princípio básico de teoria da medida, toda a informação a respeito de um campo aleatório está contido em sua distribuição conjunta em $E^{S}$. Portando podemos (e vamos) assumir que cada campo aleatório é dado em sua "versão canônica". Isto é, vamos concordar em fazer as seguintes escolhas específicas:

a. $\Omega=E^{S}=\left\{\left(\omega_{i}\right)_{i \in S}: \omega_{i} \in E\right\}$ o conjunto de todas as configurações $\omega$ de spins. Consideraremos em $\Omega$ a sigma álgebra produto $\mathscr{F}=\mathscr{E}^{\mathscr{S}}$. Denotaremos por $\sigma_{i}: \Omega \rightarrow E$ a projeção sobre a i-ésima coordenada, isto é, $\sigma_{i}(\omega)=\omega_{i}$.

b. Vamos introduzir notações para uso posterior. Para cada $\Lambda \subset S$ denote por $\sigma_{\Lambda}: \Omega \rightarrow E^{\Lambda}$ a projeção sobre as coordenadas em $\Lambda$, isto é, $\sigma_{\Lambda}(\omega)=\left(\omega_{i}\right)_{i \in \Lambda}$. Quando $\Lambda \subset \Delta \subset S$ vamos abusar da notação e usaremos o mesmo símbolo $\sigma_{\Lambda}$ para a projeção de $E^{\Delta}$ sobre $E^{\Lambda}$.

c. Se $\omega \in E^{\Lambda}$ e $\xi \in E^{\Delta \backslash \Lambda}$ a notação $\omega \xi \in E^{\Delta}$ é definido pelas seguintes propriedades, $\sigma_{\Lambda}(\omega \xi)=\omega$ e $\sigma_{\Delta \backslash \Lambda}(\omega \xi)=\xi$.

d. Considere o conjunto $\mathscr{S}=\{\Lambda \subset S: 0<|\Lambda|<\infty\}$ onde $|\Lambda|$ denota a cardinalidade do conjunto $\Lambda$. Por definição $\mathscr{F}$ é a menor sigma álgebra contendo os cilindros

$$
\left\{\sigma_{\Lambda} \in A\right\} \equiv\left\{\sigma_{\Lambda} \in A, \quad \Lambda \in \mathscr{S}, A \in \mathscr{E}^{\Lambda}\right\} .
$$


Para cada $\Delta \subset S$ podemos considerar a sigma álgebra $\mathscr{F}_{\Delta}$ de "todos os eventos ocorrendo em $\Delta$ " gerada pelos eventos

$$
\left\{\sigma_{\Lambda} \in A\right\} \equiv\left\{\sigma_{\Lambda} \in A, \Lambda \in \mathscr{S}, \Lambda \subset \Delta, A \in \mathscr{E} \Lambda\right\}
$$

Note que para cada $\Delta \in \mathscr{S}$ a sigma álgebra $\mathscr{F}_{\Delta}$ é uma sub sigma álgebra de $\mathscr{F}$.

4. A seguir introduziremos notações gerais envolvendo medidas e núcleos de medidas.

a. Seja $(X, \mathscr{X})$ um espaço mensurável. Denote por $\mathscr{M}(X, \mathscr{X})$ o conjunto de todas as medidas $\sigma$-finitas $\mu$ sobre o espaço $(X, \mathscr{X})$ com $\mu(X)>0$. Use $\mathscr{P}(X, \mathscr{X})$ para denotar o subconjunto de $\mathscr{M}(X, \mathscr{X})$ formado pelas probabilidades sobre $(X, \mathscr{X})$.

b. Frequentemente usaremos a notação $\mu(f)=\int f d \mu$ para a integral de uma função mensurável $f: X \rightarrow \mathbb{R}$ com respeito a uma medida $\mu$ sobre $(X, \mathscr{X})$.

c. Se $\mathscr{B}$ é uma subsigma álgebra de $\mathscr{X}$ e $\mu \in \mathscr{P}(X, \mathscr{X})$ vamos escrever $\mu(f \mid \mathscr{B})=\mathbb{E}_{\mu}(f \mid \mathscr{B})$ para designar a $\mu$-esperança condicional de $f$ dado à sigma álgebra $\mathscr{B}$. Em particular escreveremos $\mu(A \mid \mathscr{B}) \equiv \mu\left(1_{A} \mid \mathscr{B}\right)$ para denotar a probabilidade de um evento $A$ dado $\mathscr{B}$.

d. Seja $f: X \rightarrow \mathbb{R}$ uma função mensurável não negativa, denotaremos por $f \mu$ a medida com densidade de Radon-Nikodym $f$ com respeito a $\mu$, mais explicitamente

$$
f \mu(A) \equiv \mu\left(f 1_{A}\right)=\int_{A} f d \mu, \quad A \in \mathscr{X} .
$$

Se $\nu$ é outra medida no espaço mensurável $(X, \mathscr{X})$ então a notação $\nu<<\mu$ significa que $\nu$ é absolutamente contínua com relação a $\mu$.

\section{Núcleos de medida e núcleos de probabilidade.}

a. Seja $(Y, \mathscr{Y})$ um segundo espaço de medida. Uma função $\pi: \mathscr{X} \times Y \rightarrow[0, \infty]$ é dita um núcleo de medida de $(Y, \mathscr{Y})$ para $(X, \mathscr{X})$ (ou mais sucintamente de $\mathscr{Y}$ para $\mathscr{X}$ ) quando tem as seguintes propriedades

(i) $\pi(\cdot \mid y)$ é uma medida em $(X, \mathscr{X})$ para todo $y \in Y$;

(ii) $\pi(A \mid \cdot)$ é $\mathscr{Y}$-mensurável para todo $A \in \mathscr{X}$

em particular quando $\pi(X \mid y)=1$ para todo $y \in Y$ dizemos que $\pi$ é um núcleo de probabilidade de $(Y, \mathscr{Y})$ para $(X, \mathscr{X})$

b. Se $\varphi$ é uma aplicação mensurável de $Y$ para $X$ então a função $(A, y) \mapsto 1_{A} \circ \varphi(y)$ é um núcleo do probabilidade de $\mathscr{Y}$ para $\mathscr{X}$.

c. Um núcleo de probabilidade $\pi$ de $\mathscr{Y}$ para $\mathscr{X}$ associa naturalmente cada a medida de $\mu \in \mathscr{M}(Y, \mathscr{Y})$ uma medida $\mu \pi$ em $\mathscr{M}(X, \mathscr{X})$ definida do seguinte modo

$$
\mu \pi(A) \equiv \int \pi(A \mid \cdot) d \mu \equiv \int \pi(A \mid x) d \mu(x), \quad A \in \mathscr{X} .
$$


d. Seja $f: X \rightarrow \mathbb{R}$ então associamos naturalmente função $\pi f: Y \rightarrow \mathbb{R}$ dada por

$$
\pi f(y) \equiv \int \pi(d x \mid y) f(x)
$$

desde que esteja bem definida.

e. Seja $f: X \rightarrow \mathbb{R}$ uma funçao mensurável positiva. Denote por $f \pi$ o núcleo de medida de $\mathscr{Y}$ para $\mathscr{X}$ dado por

$$
f \pi(A \mid \cdot) \equiv \int \pi(d x \mid \cdot) f(x) 1_{A} .
$$

f. Seja $(Z, \mathscr{Z})$ é um terceiro espaço mensurável. Então definimos a composição de dois núcleos de probabilidade $\pi_{1}, \pi_{2}$ de $\mathscr{Z}$ para $\mathscr{Y}$ e $\mathscr{Y}$ para $\mathscr{X}$ respectivamente como sendo

$$
\pi_{1} \circ \pi_{2}(A \mid z) \equiv \int \pi_{1}(d y \mid z) \pi(A \mid y) .
$$

g. Seja $\mathscr{B}$ uma subsigma algebra de $\mathscr{X}$. Um núcleo de probabilidade de $\mathscr{B}$ para $\mathscr{X}$ é próprio se

$$
\pi(A \cap B \mid \cdot)=\pi(A \mid \cdot) \cdot 1_{B} \quad A \in \mathscr{X}, B \in \mathscr{B} .
$$

Esta condição claramente implica que $\pi(f g)=\pi(f) \cdot g$ sempre que $f$ for $\mathscr{X}$ mensurável e $g$ for uma função limitada $\mathscr{B}$-mensurável.

Proposição A.1.1. Seja $\pi$ é um núcleo de probabilidade de $\mathscr{B}$ para $\mathscr{X}$. A propriedade de o núcleo de probabilidade $\pi$ ser próprio é equivalente a dizer que $\pi(B \mid \cdot)=1_{B}, B \in \mathscr{B}$.

Demonstração. Sejam $A \in \mathscr{X}$ e $B \in \mathscr{B}$, então temos que

$$
\begin{aligned}
\pi(A \cap B \mid \cdot) & \leq \min \{\pi(A \mid \cdot), \pi(B \mid \cdot)\} \\
& =\min \left\{\pi(A \mid \cdot), 1_{B}\right\}=\pi(A \mid \cdot) \cdot 1_{B}
\end{aligned}
$$

usando o mesmo raciocínio verifica-se que $\pi(A \backslash B \mid \cdot) \leq \pi(A \mid \cdot) \cdot 1_{X \backslash B}$. Agora, suponha por absurdo que $\pi(A \cap B \mid \cdot)<\pi(A \mid \cdot) \cdot 1_{B}$, então teremos que

$$
\pi(A \mid \cdot)=\pi(A \cap B \mid \cdot)+\pi(A \backslash B \mid \cdot)<\pi(A \mid \cdot) \cdot 1_{B}+\pi(A \mid \cdot) \cdot 1_{X \backslash B}=\pi(A \mid \cdot)
$$

o que é um absurdo.

Proposição A.1.2. Seja $(X, \mathscr{X})$ um espaço mensurável, $\mathscr{B}$ uma sub sigma álgebra de $\mathscr{X}$, e $\pi$ um núcleo de de probabilidade próprio de $\mathscr{B}$ para $\mathscr{X}$ e $\mu \in \mathscr{P}(X, \mathscr{X})$. Então

$$
\mu(A \mid \mathscr{B})=\pi(A \mid \cdot) \quad \mu-q . t . p \quad \forall A \in \mathscr{X}
$$

se e somente se $\mu \pi=\mu$. 
Demonstração. De fato, suponha inicialmente que $\mu \pi=\mu$. Sejam $A \in \mathscr{X}$ e $B \in \mathscr{B}$ então temos que

$$
\mu(A \cap B)=\mu \pi(A \cap B)=\int \pi(A \cap B \mid \cdot) d \mu=\int 1_{B} \pi(A \mid \cdot) d \mu=\int_{B} \pi(A \mid \cdot) d \mu .
$$

Reciprocamente suponha que $\mu(A \mid \mathscr{B})=\pi(A \mid \cdot) \quad \mu$-q.t.p $\forall A \in \mathscr{X}$ então temos que

$$
\mu(A)=\int \mu(A \mid \mathscr{B}) d \mu=\int \pi(A \mid \cdot) d \mu=\mu \pi(A) .
$$

\section{A.2 EsPeCIFICAÇÕES}

De acordo com nossas intenções físicas estamos interessados em campos aleatórios exibindo um certo tipo de dependência em particular. Para especificar esse tipo de dependência nós vamos fazer uma analogia com processos de de Markov: nós vamos olhar para as distribuições condicionais de certas coleções de spins quando os valores de todos os outros spins são dados e vamos requerer que quase certamente essas probabilidades sejam prescritas por certos núcleos de probabilidade. Entretanto, em contraste com as definições de cadeia de Markov nós não usaremos conceitos de futuro e passado, pois estes necessitam de uma ordem linear em $S$. Ao invés disso usaremos um procedimento introduzido por Dobrushin, Lanford e Ruelle que consiste em descrever as distribuições condicionais de todas as coleções finitas de spins. Ou seja vamos olhar as distribuições condicionais relativas às sigma álgebras externas $\mathscr{T}_{\Lambda}=\mathscr{F}_{S \backslash \Lambda}$, onde $\Lambda$ percorre o conjunto $\mathscr{S}$ de todos os subconjuntos finitos de $S$. (Por definição $\mathscr{T}_{\Lambda}$ é a sigma álgebra que contém toda a informação sobre "as informações de bordo") Tomando a família $\left(\mathscr{T}_{\Lambda}\right)_{\Lambda \in \mathscr{S}}$ de sigmas álgebras como base nos podemos proceder como no caso das cadeias de Markov. Um tipo de dependência para um campo aleatório é especificada por uma família $\gamma=\left(\gamma_{\Lambda}\right)_{\Lambda \in \mathscr{S}}$ de núcleos de probabilidade $\gamma_{\Lambda}$ de $\left(\Omega, \mathscr{T}_{\Lambda}\right)$ para $(\Omega, \mathscr{F})$. Dizemos que um campo aleatório $\mu$ exibe o tipo de dependência especificado por $\gamma$ se satisfaz a seguinte condição

$$
\mu\left(A \mid \mathscr{T}_{\Lambda}\right)=\gamma_{\Lambda}(A \mid \cdot) \quad \mu \text {-q.t.p } \forall A \in \mathscr{F}, \quad \forall \Lambda \in \mathscr{S} \text {. }
$$

Em outras palavras esta condição significa que para cada $\Lambda$ finito o núcleo dado $\gamma_{\Lambda}$ é uma distribuição condicional regular de $\mu$ relativa à sigma álgebra $\mathscr{T}_{\Lambda}$. Suponha agora que temos dada uma família $\gamma$ como anteriormente. Suponha ainda $\gamma$ ainda é razóavel, no sentido de que existe um campo aleatório que satisfaz A.1 então $\gamma$ é obrigado a satisfazer duas condições. Com efeito sejam $\Lambda$ e $\Delta$ em $\mathscr{S} \operatorname{com} \Lambda \subset \Delta, A \in \mathscr{F}$ e $B \in \mathscr{T}_{\Lambda}$ dados, então pelas propriedades da probabilidade condicional temos que

$$
\gamma_{\Lambda}(A \cap B \mid \cdot)=\mu\left(A \cap B \mid \mathscr{T}_{\Lambda}\right)=\mu\left(A \mid \mathscr{T}_{\Lambda}\right) \cdot 1_{B}=\gamma_{\Lambda}(A \mid \cdot) \cdot 1_{B}, \quad \mu \text {-q.t.p }
$$

e que

$$
\gamma_{\Delta} \gamma_{\Lambda}(A \mid \cdot)=\mu\left(\mu\left(A \mid \mathscr{T}_{\Lambda}\right) \mid \mathscr{T}_{\Delta}\right)=\mu\left(A \mid \mathscr{T}_{\Delta}\right)=\gamma_{\Delta}(A \mid \cdot), \quad \mu \text {-q.t.p }
$$


onde para obter a segunda igualdade usamos que $\mathscr{T}_{\Delta} \subset \mathscr{T}_{\Lambda}$ e propriedades da esperança condicional. Então temos que $\gamma_{\Lambda}$ é "própria quase certamente"e $\gamma_{\Lambda}$ e $\gamma_{\Delta}$ são "consistentes quase certamente". Entretanto, queremos que $\gamma$ seja razoável sem conhecer ou requerer previamente nenhuma propriedade especial sobre o suporte dos campos aleatórios $\mu$ satisfazendo A.1. Sendo assim vamos requerer que as propriedades precedentes são satisfeitas em todos os pontos ao invés de $\mu$-q.t.p. Temos então a seguinte definição:

Definição A.2.1. Uma especificação com espaço de parâmetros $S$ e espaço de estados $(E, \mathscr{E})$ é uma familia $\gamma=\left(\gamma_{\Lambda}\right)_{\Lambda \in S}$ de núcleos de probabilidade próprios satisfazendo a condição de consistência $\gamma_{\Delta} \circ \gamma_{\Lambda}=\gamma_{\Delta}$ sempre que $\Lambda \subset \Delta$.

Os campos aleatórios no conjunto

$$
\mathscr{G}(\gamma)=\left\{\mu \in \mathscr{P}(\Omega, \mathscr{F}): \mu\left(A \mid \mathscr{T}_{\Lambda}\right)=\gamma_{\Lambda}(A \mid \cdot), \quad \mu \text {-q.t.p, } \quad \forall A \in \mathscr{F}, \Lambda \in \mathscr{S}\right\}
$$

são ditos especificados por $\gamma$.

Observação A.2.2. Note que uma especificação $\gamma=\left(\gamma_{\Lambda}\right)_{\Lambda \in S}$ sempre satisfaz a reciproca da relação de consistência $\gamma_{\Lambda} \circ \gamma_{\Delta}=\gamma_{\Delta}$ sempre que $\Lambda \subset \Delta$. Isso decorre do fato de que $\gamma_{\Lambda}$ é próprio e que $\mathscr{T}_{\Delta} \subset \mathscr{T}_{\Lambda}$. Mais explicitamente temos que

$$
\gamma_{\Lambda} \circ \gamma_{\Delta}(A \mid \cdot)=\gamma_{\Lambda}(1 \cdot \underbrace{\gamma_{\Delta}(A \mid \cdot)}_{\mathscr{T}_{\Delta} \text { mensurável }})=\gamma_{\Delta}(A \mid \cdot) \cdot \gamma_{\Lambda}(1)=\gamma_{\Delta}(A \mid \cdot)
$$

onde usamos acima que $\gamma_{\Lambda}$ é próprio e $\mathscr{T}_{\Delta} \subset \mathscr{T}_{\Lambda}$.

Proposição A.2.3. Seja $\gamma$ uma especificação e $\mu \in \mathcal{M}(\Omega)$. Então $\mu \in \mathscr{G}(\gamma)$ se e somente se $\mu \gamma_{\Lambda}=\mu$ para todo $\Lambda \in \mathscr{S}$.

Demonstração. Corolário imediato da Proposição A.1.2.

\section{A.3 $\lambda$-ESPECIFICAÇÕES}

Já tendo introduzido a noção de especificação precisamos estabelecer que especificações existem.

Proposição A.3.1. Seja $\lambda \in \mathscr{P}(E, \mathscr{E})$ dado. Defina

$$
\lambda_{\Lambda}(A \mid \cdot)=\lambda^{\Lambda} \times \delta_{\omega_{S \backslash \Lambda}}(A)=\lambda^{\Lambda}\left\{\xi \in E^{\Lambda}: \xi \omega_{S \backslash \Lambda} \in A\right\}
$$

onde $\varnothing \neq A \subset S, A \in \mathscr{F}, \omega \in \Omega$. Então cada $\lambda_{\Lambda}$ é um núcleo de probabilidade próprio de $\left(\Omega, \mathscr{T}_{\Lambda}\right)$ para $(\Omega, \mathscr{F})$ e para quaisquer $\Lambda, \Delta \subset S$ não vazios temos que $\lambda_{\Delta} \circ \lambda_{\Lambda}=\lambda_{\Delta \cup \Lambda}$. Temos em particular que $\lambda=\left(\lambda_{\Lambda}\right)_{\Lambda \in \mathscr{S}}$ é uma especificação que satisfaz $\mathscr{G}(\lambda)=\left\{\Lambda^{S}\right\}$.

Demonstração. Para cada $\omega \in \Omega$ temos que $\lambda_{\Lambda}(A \mid \omega)$ é uma probabilidade pois é o produto de duas probabilidades, $\lambda^{\Lambda}$ e $\delta_{\omega_{S \backslash \Lambda}}$. Seja $f$ uma função mensurável limitada em $\Omega$, note que 
podemos escrever $\Omega=E^{\Lambda} \times E^{S \backslash \Lambda}$, assim podemos pensar em $f$ como uma função de duas variáveis $(\xi, \eta)$. Então temos que

$\gamma_{\Lambda} f(\omega)=\int f(\xi \eta) \lambda^{\Lambda}(d \xi) \times \delta_{\omega_{S \backslash \Lambda}}(d \eta)=\int\left(\int f(\xi \eta) \delta_{\omega_{S \backslash \Lambda}}(d \eta)\right) \lambda^{\Lambda}(d \xi)=\int f\left(\xi \omega_{S \backslash \Lambda}\right) \lambda^{\Lambda}(d \xi)$

onde para a segunda igualdade usamos o teorema de Fubinni. Segue também do teorema de Fubinni que $\gamma_{\Lambda} f$ é uma função mensurável da segunda coordenada, mais precisamente, $\gamma_{\Lambda} f$ é $\mathscr{T}_{\Lambda}$ mensurável. Escolhendo em particular $f=1_{A}, A \in \mathscr{F}$, temos que

$$
\gamma_{\Lambda}(A \mid \cdot)=\gamma_{\Lambda} 1_{A}=\int 1_{A}\left(\xi \omega_{S \backslash \Lambda}\right) \lambda^{\Lambda}(d \xi)=\lambda^{\Lambda}\left\{\xi \in E^{\Lambda}: \xi \omega_{S \backslash \Lambda} \in A\right\}
$$

concluímos que $\lambda_{\Lambda}$ é um núcleo de probabilidade.

Precisamos mostrar que $\lambda_{\Lambda}$ é próprio. Sejam $A \in \mathscr{F}, B \in \mathscr{T}_{\Lambda}$ então temos que

$$
\begin{aligned}
\lambda_{\Lambda}(A \cap B \mid \cdot) & =\int 1_{A \cap B}\left(\xi \omega_{S \backslash \Lambda}\right) \lambda^{\Lambda}(d \xi)=\int 1_{A}\left(\xi \omega_{S \backslash \Lambda}\right) 1_{B}\left(\xi \omega_{S \backslash \Lambda}\right) \lambda^{\Lambda}(d \xi) \\
& =1_{B}\left(\xi \omega_{S \backslash \Lambda}\right) \cdot \int 1_{A}\left(\xi \omega_{S \backslash \Lambda}\right) \lambda^{\Lambda}(d \xi)=1_{B}(\omega) \cdot \lambda_{\Lambda}(A \mid \cdot)
\end{aligned}
$$

onde a penúltima igualdade se justifica pois sendo $1_{B}$ uma função $\mathscr{T}_{\Delta}$ mensurável ela deve ser constante na variável $\xi$. Portanto $\lambda_{\Lambda}$ é um núcleo próprio. Agora sejam $\Lambda, \Delta \subset S$ não vazios. Temos para cada função mensurável $f$ e todo $\omega \in \Omega$ que

$$
\begin{aligned}
\lambda_{\Delta} \circ \lambda_{\Lambda} f(\omega) & =\int\left(\lambda_{\Lambda} f\right) \lambda_{\Delta}(d \xi \eta \mid \omega)=\int \lambda_{\Lambda} f\left(\xi \omega_{S \backslash \Delta}\right) \lambda^{\Delta}(d \xi) \\
& =\int \lambda^{\Delta}(d \xi) \int f\left(\eta\left(\xi \omega_{S \backslash \Delta}\right)_{S \backslash \Lambda}\right) \lambda^{\Lambda}(d \eta)=\int \lambda^{\Delta}(d \xi) \underbrace{\int f\left(\eta \xi_{\Delta \backslash \Lambda} \omega_{S \backslash \Delta \cup \Lambda}\right) \lambda^{\Lambda}(d \eta)}_{\mathscr{F} \Delta \backslash \Lambda \text { - mensuravel }} \\
& =\int \lambda^{\Delta \backslash \Lambda}(d \xi) \int f\left(\eta \xi_{\Delta \backslash \Lambda} \omega_{S \backslash \Delta \cup \Lambda}\right) \lambda^{\Lambda}(d \eta)=\int f\left(\eta \xi_{\Delta \backslash \Lambda} \omega_{S \backslash \Delta \cup \Lambda}\right) \lambda^{\Delta \cup \Lambda}(d \eta \xi) \\
& =\lambda_{\Delta \cup \Lambda} f(\omega) .
\end{aligned}
$$

Observação A.3.2. Para qualquer medida $\lambda \in \mathscr{M}(E, \mathscr{E})$ vamos usar a notação $\lambda=\left(\lambda_{\Lambda}\right)_{\Lambda \in \mathscr{S}}$ para denotar a familia de núcleos de medida $\lambda_{\Lambda}$ de $\mathscr{T}_{\Lambda}$ para $\mathscr{F}$ definida por

$$
\lambda_{\Lambda}(\cdot \mid \omega)=\lambda^{\Lambda} \times \delta_{\omega_{S \backslash \Lambda}}, \quad \Lambda \in \mathscr{S}, \omega \in \Omega .
$$

Definição A.3.3 ( $\lambda$-modificações, $\lambda$-especificações). Seja $\lambda \in \mathscr{M}(E, \mathscr{E})$. Uma $\lambda$ - modificação é uma família $\rho=\left(\rho_{\Lambda}\right)_{\Lambda \in \mathscr{S}}$ de funções mensuráveis $\rho: \Omega \rightarrow[0, \infty)$ tal que a família $\rho \lambda=$ $\left(\rho_{\Lambda} \lambda_{\Lambda}\right)_{\lambda \in \mathscr{S}}$ é uma especificação. Uma $\lambda$-especificação é uma especificação $\gamma$ da forma $\gamma=\rho \lambda$ para alguma $\lambda$-modificação $\rho$. Uma $\lambda$-modificação $\rho$ ( e a $\lambda$-especificação associada $\rho \lambda) e^{-}$ chamada positiva se $\rho_{\Lambda}>0$ para todo $\Lambda \in \mathscr{S}$. 
Observação A.3.4. A seguir temos algumas observações a respeito do exposto acima. a. Cada núcleo de probabilidade $\rho_{\Lambda} \lambda_{\Lambda}$ é próprio pois $\lambda_{\Lambda}$ o é.

b. Pela observação anterior uma família $\left(\rho_{\Lambda}\right)_{\Lambda \in \mathscr{S}}$ de funções mensuráveis é uma $\lambda$-modificação se e somente se os núcleos $\rho_{\Lambda} \lambda_{\Lambda}$ são normalizados, i.e, $\lambda_{\Lambda} \rho_{\Lambda}=1$, e consistente, i.e, para todo $A \in \mathscr{F}$ temos que

$$
\left(\rho_{\Delta} \lambda_{\Delta}\right) \circ\left(\rho_{\Lambda} \lambda_{\Lambda}\right) 1_{A}=\lambda_{\Delta}\left(\rho_{\Delta} \cdot \lambda_{\Lambda}\left(\rho_{\Lambda} 1_{A}\right)\right)=\lambda_{\Delta}\left(\rho_{\Delta} 1_{A}\right)=\rho_{\Delta} \lambda_{\Delta} 1_{A}
$$

para todos $\Lambda, \Delta \in \mathscr{S} \operatorname{com} \Lambda \subset \Delta$.

c. Seja $\gamma=\rho \lambda$ uma $\lambda$-especificação. Então temos que

$$
\mathscr{G}(\gamma)=\left\{\mu \in \mathscr{P}(\Omega, \mathscr{F}): \mu=\rho_{\Lambda}\left(\mu \lambda_{\Lambda}\right) \quad \forall \Lambda \in \mathscr{S}\right\},
$$

de fato,

$$
\begin{aligned}
\left(\mu \gamma_{\Lambda}\right) 1_{A} & =\int \mu(d \omega) \gamma_{\Lambda} 1_{A}=\int \mu(d \omega) \int_{A} \lambda_{\Lambda}(d \xi \mid \omega) \rho_{\Lambda}(\xi) \\
& =\left(\mu \lambda_{\Lambda}\right)\left(1_{A} \rho_{\Lambda}\right)=\rho_{\Lambda}\left(\mu \lambda_{\Lambda}\right) 1_{A}
\end{aligned}
$$

portanto $\mu \gamma_{\Lambda}=\mu$ se e somente se $\mu=\rho_{\Lambda}\left(\mu \lambda_{\Lambda}\right)$ para todo $\Lambda \in \mathscr{S}$.

d. Seja $\gamma$ uma especificação. Uma condição necessária e suficiente para que $\gamma$ seja uma $\lambda$ especificação é que

$$
\gamma_{\Lambda}(\cdot \mid \omega)<<\lambda_{\Lambda}(\cdot \mid \omega), \quad \forall \Lambda \in \mathscr{S}, \forall \omega \in \Omega .
$$

Em geral essa condição não é suficiente para que $\gamma$ seja uma $\lambda$ especificação. Isso acontece pois as densidades $\rho_{\Lambda}$ provenientes do teorema de Radon Nikodym são, a priori, apenas parcialmente mensuráveis, no seguinte sentido, $\xi \mapsto \rho_{\Lambda}\left(\xi_{\Lambda} \omega_{S \backslash \Lambda}\right)$ é mensurável para todo $\omega \in \Omega$ fixado. Entretanto se a sigma álgebra $\mathscr{E}$ é contavelmente gerada então um "contrutive martingale aproach"ao teorema de Radon Nikodym mostra que $\gamma$ é uma $\lambda$-espeficicação sempre que $\gamma$ for absolutamente continuo com respeito a $\lambda$ no sentido definido acima.

Proposição A.3.5. Seja $\lambda \in \mathscr{M}(E, \mathscr{E})$ e $\rho=\left(\rho_{\Lambda}\right)_{\Lambda \in \mathscr{S}}$ uma família de funções mensuráveis não negativas em $\Omega \operatorname{com} \lambda_{\Lambda} \rho_{\Lambda}=1$. Então as seguintes afirmações são equivalentes:

(a) $\rho$ é uma $\lambda$-modificação.

(b) Para todos $\Lambda, \Delta \in \mathscr{S} \operatorname{com} \Lambda \subset \Delta$ e todo $\omega \in \Omega$ temos $\lambda_{\Delta}(\cdot \mid \omega)$-q.t.p que

$$
\rho_{\Delta}=\rho_{\Lambda} \cdot \lambda_{\Lambda} \rho_{\Delta}
$$

(c) Para todo $\Lambda, \Delta \in \mathscr{S}$ com $\Lambda \subset \Delta$, todo $\alpha \in \Omega$ e $\lambda_{\Delta \backslash \Lambda}(\cdot \mid \alpha)$ quase todo $\omega \in \Omega$ temos que

$$
\rho_{\Delta}(\xi) \rho_{\Lambda}(\eta)=\rho_{\Delta}(\eta) \rho_{\Lambda}(\xi)
$$

para $\lambda_{\Lambda}(\cdot \mid \omega) \times \lambda_{\Lambda}(\cdot \mid \omega)$ quase todo $(\xi, \eta) \in \Omega \times \Omega$. 
Demonstração. Prova de $(a) \Leftrightarrow(b)$ : Note que $\rho$ vai ser uma $\lambda$-modificação se e somente se $\rho \lambda$ satisfaz as condições de consistência, i.e, para todos $\Lambda, \Delta \in \mathscr{S}, \Lambda \subset \Delta$ temos $\gamma_{\Delta} \circ \gamma_{\Lambda}=$ $\left(\rho_{\Delta} \lambda_{\Delta}\right) \circ\left(\rho_{\Lambda} \lambda_{\Lambda}\right)=\rho_{\Delta} \lambda_{\Delta}=\gamma_{\Delta}$. Seja $f$ uma função mensurável limitada em $\Omega$, temos então que $\rho \lambda$ é uma especificação se e somente se

$$
\begin{aligned}
\lambda_{\Delta}\left(f \rho_{\Delta}\right) & =\gamma_{\Delta} f=\gamma_{\Delta} \circ \gamma_{\Lambda} f=\gamma_{\Delta}\left(\gamma_{\Lambda} f\right) \\
& =\lambda_{\Delta}\left(\lambda_{\Lambda} f \rho_{\Lambda} \cdot \rho_{\Delta}\right)
\end{aligned}
$$

agora usando que $\lambda_{\Delta}=\lambda_{\Delta} \circ \lambda_{\Lambda}$ temos que

$$
\begin{aligned}
\lambda_{\Delta}\left(f \rho_{\Delta}\right) & =\lambda_{\Delta} \circ \lambda_{\Lambda}\left(\lambda_{\Lambda} f \rho_{\Lambda} \cdot \rho_{\Delta}\right)=\lambda_{\Delta}\left(\lambda_{\Lambda}(\underbrace{\lambda_{\Lambda} f \rho_{\Lambda}}_{\mathscr{T}_{\Lambda} \text { mens. }} \cdot \rho_{\Delta})\right) \\
& =\lambda_{\Delta}(\lambda_{\Lambda} f \rho_{\Lambda} \cdot \underbrace{\lambda_{\Lambda} \rho_{\Delta}}_{\mathscr{T}_{\Lambda} \text { mens. }})=\lambda_{\Delta}\left(\lambda_{\Lambda}\left(f \rho_{\Lambda} \cdot \lambda_{\Lambda} \rho_{\Delta}\right)\right. \\
& =\lambda_{\Delta} \circ \lambda_{\Lambda}\left(f \rho_{\Lambda} \cdot \lambda_{\Lambda} \rho_{\Delta}\right)=\lambda_{\Delta}\left(f \rho_{\Lambda} \cdot \lambda_{\Lambda} \rho_{\Delta}\right)
\end{aligned}
$$

portanto, temos do Teorema de Radon Nikodym que $\rho \lambda$ satisfaz as condições de consistência se e somente se $\rho_{\Delta}=\rho_{\Lambda} \cdot \lambda_{\Lambda} \rho_{\Delta}$ para $\lambda_{\Delta}$ quase todo ponto.

Prova de $(b) \Rightarrow(c)$ : Seja $\alpha \in \Omega$. Então, $(b)$ implica que

$$
\begin{aligned}
0 & =\lambda_{\Delta}\left(\left|\rho_{\Delta}-\rho_{\Lambda} \cdot \lambda_{\Lambda} \rho_{\Delta}\right|\right)(\alpha) \\
& =\lambda_{\Delta \backslash \Lambda} \circ \lambda_{\Lambda}\left(\left|\rho_{\Delta}-\rho_{\Lambda} \cdot \lambda_{\Lambda} \rho_{\Delta}\right|\right)(\alpha) \\
& =\int \lambda_{\Delta \backslash \Lambda}(d \omega \mid \alpha) \lambda_{\Lambda}\left(\left|\rho_{\Delta}-\rho_{\Lambda} \cdot \lambda_{\Lambda} \rho_{\Delta}\right|\right)(\omega)
\end{aligned}
$$

segue do exposto acima que para $\lambda_{\Delta \backslash \Lambda}$ quase todo $\omega$ temos $\lambda_{\Lambda}\left(\left|\rho_{\Delta}-\rho_{\Lambda} \cdot \lambda_{\Lambda} \rho_{\Delta}\right|\right)=0$, ou seja, $\lambda_{\Delta \backslash \Lambda}$ quase todo $\omega$

$$
\rho_{\Delta}=\rho_{\Lambda} \cdot \lambda_{\Lambda} \rho_{\Delta}
$$

para $\lambda_{\Lambda}(\cdot \mid \omega)$ quase todo ponto. Então fixado $\omega$ temos que $\rho_{\Delta}(\xi)=\rho_{\Lambda}(\xi) \cdot \lambda_{\Lambda} \rho_{\Delta}(\omega)$, multiplicando ambos os membros por $\rho_{\Lambda}(\eta)$ obtemos que

$$
\rho_{\Delta}(\xi) \rho_{\Lambda}(\eta)=\rho_{\Lambda}(\eta) \rho_{\Lambda}(\xi) \lambda_{\Lambda} \rho_{\Delta}(\omega)=\rho_{\Lambda}(\xi) \underbrace{\rho_{\Lambda}(\eta) \lambda_{\Lambda} \rho_{\Delta}(\omega)}_{\rho_{\Delta}(\eta)}=\rho_{\Lambda}(\xi) \rho_{\Delta}(\eta)
$$

é claro que esse procedimento só vale para $\lambda_{\Lambda}(\cdot \mid \omega) \times \lambda_{\Lambda}(\cdot \mid \omega)$ quase todo $(\xi, \eta) \in \Omega \times \Omega$.

Prova de $(c) \Rightarrow(b)$ : Basta observar que $\lambda_{\Delta \backslash \Lambda} \circ \lambda_{\Lambda}=\lambda_{\Delta}$ e integrar em $\eta$.

\section{PRÉ-MODIFICAÇÕES.}

Definição A.3.6. Uma família de funções $h=\left(h_{\Lambda}\right)_{\Lambda \in \mathscr{S}}$ de funções mensuráveis $h_{\Lambda}: \Omega \rightarrow$ $[0, \infty)$ é dita uma pré-modificação se

$$
h_{\Delta}(\xi) h_{\Lambda}(\eta)=h_{\Lambda}(\xi) h_{\Delta}(\eta)
$$

para todos $\Lambda, \Delta \in \mathscr{S} \operatorname{com} \Lambda \subset \Delta$ e todos $\xi, \eta \in \Omega \operatorname{com} \xi_{S \backslash \Lambda}=\eta_{S \backslash \Lambda}$. 
Proposição A.3.7. Seja $h$ uma pré-modificação e $\lambda \in \mathscr{M}(E, \mathscr{E})$ tal que $0<\lambda_{\Lambda} h_{\Lambda}<\infty$ para todo $\Lambda \in \mathscr{S}$. Então $\rho=\left(h_{\lambda} / \lambda_{\Lambda} h_{\Lambda}\right)_{\Lambda \in \mathscr{S}}$ é uma $\lambda$-modificação.

Demonstração. Visto que $\lambda_{\Lambda} h_{\Lambda}$ temos que $\mathscr{T}_{\Lambda}$-mensurável e que $\lambda_{\Lambda} \rho_{\Lambda}=1$, sendo assim $\rho$ também é uma pré-modificação. Agora note que para cada $\omega \in \Omega$ fixado a medida $\lambda_{\Lambda}(\cdot \mid \omega)$ está suportada no conjunto $\left\{\xi \in \Omega ; \xi_{S \backslash \Lambda}=\omega_{S \backslash \Lambda}\right\}$, portanto $\lambda_{\Lambda}(\cdot \mid \omega) \times \lambda_{\Lambda}(\cdot \mid \omega)$ está suportada em $\left.\{(\xi, \eta) \in \Omega \times \Omega): \xi_{S \backslash \Lambda}=\eta_{S \backslash \Lambda}=\omega_{S \backslash \Lambda}\right\}$. O resultado segue diretamente do teorema anterior.

Observação: A expressão "está suportada em..." quer dizer que qualquer coisa fora do "suporte" tem medida 0 .

Teorema A.3.8. Seja $\lambda \in \mathscr{M}(E, \mathscr{E})$ e suponha que $\gamma$ é uma espeficação que tem a seguinte propriedade: para cada $i \in S$ existe uma função mensurável $\rho_{\{i\}}>0$ em $\Omega$ tal que $\gamma_{\{i\}}=\rho_{\{i\}} \lambda_{\{i\}}$. Então existe uma $\lambda$-modificação $\rho$ com $\gamma=\rho \lambda$, além disso $\gamma$ é unicamente determinada por $\left(\rho_{\{i\}}\right)_{i \in S}$ e $\lambda$. Temos também que

$$
\begin{aligned}
\mathscr{G}(\gamma) & =\left\{\mu \in \mathscr{P}(\Omega, \mathscr{F}): \mu \gamma_{\{i\}}=\mu \quad \forall i \in S\right\} \\
& =\left\{\mu \in \mathscr{P}(\Omega, \mathscr{F}): \mu=\rho_{\{i\}}\left(\mu \lambda_{\{i\}}\right) \quad \forall i \in S\right\} .
\end{aligned}
$$

Demonstração. Tendo em vista o a Observação A.3.4 item (b) podemos considerar apenas as medidas $\lambda$ satisfazendo $\lambda(E)=1$. Para cada $\Lambda \in \mathscr{S}$ vamos definir uma função mensurável $\rho_{\Lambda}$ em função dos $\left(\rho_{\{i\}}\right)_{i \in \Lambda}$ tal que

$$
\mu=\rho_{\Lambda}\left(\mu \lambda_{\Lambda}\right)
$$

para toda $\mu \in \mathscr{P}(\Omega, \mathscr{F})$ satisfazendo $\mu \gamma_{\{i\}}=\mu \forall i \in \Lambda$. Isso prova o teorema, de fato, para cada $\omega \in \Omega$ e cada $i \in \Lambda$ fixados temos que $\gamma_{\Lambda}(\cdot \mid \omega) \gamma_{\{i\}}=\gamma_{\Lambda}(\cdot \mid \omega)$ (isso ocorre pois $\gamma$ é uma especificação e portanto satisfaz as equações de compatibilidade). Voltando com $\mu=\gamma_{\Lambda}(\cdot \mid \omega)$ em A.8 obtemos que

$$
\gamma_{\Lambda}(\cdot \mid \omega)=\rho_{\Lambda}\left(\gamma_{\Lambda}(\cdot \mid \omega) \lambda_{\Lambda}\right)=\rho_{\Lambda} \lambda_{\Lambda}
$$

para todo $\omega \in \Omega$. Portanto $\gamma_{\Lambda}=\rho_{\Lambda} \lambda_{\Lambda}$. Isso mostra que (1) $\rho=\left(\rho_{\Lambda}\right)_{\Lambda \in \mathscr{S}}$ é uma $\lambda$ modificação e $\gamma=\rho \lambda$, (2) $\gamma$ é unicamente determinada por $\left(\rho_{\{i\}}\right)_{i \in S}$ e $\lambda$ e (3) se $\mu \in \mathscr{P}(\Omega, \mathscr{F})$ é tal que $\mu \gamma_{\{i\}}=\mu$ para todo $i \in S$ então $\mu=\rho_{\Lambda}\left(\mu \lambda_{\Lambda}\right)=\mu \gamma_{\Lambda}$ para todo $\Lambda \in \mathscr{S}$ e portanto $\mu \in \mathscr{G}(\gamma)$. Então vamos proceder para a prova propriamente dita do teorema. Para definir os $\rho_{\Lambda}^{\prime s}$ vamos proceder por indução em $|\Lambda|$. Então sejam $\Lambda \in \mathscr{S}$ a união dois conjuntos disjuntos $\Lambda_{1}, \Lambda_{2} \in \mathscr{S}$ para os quais $\rho_{\Lambda_{1}}$ e $\rho_{\Lambda_{2}}$ já tenham sido construídas. Definimos

$$
\rho_{\Lambda}=\left\{\begin{array}{cc}
\rho_{\Lambda_{1}} / \lambda_{\Lambda_{1}}\left(\rho_{\Lambda_{1}} \rho_{\Lambda_{2}}^{-1}\right) & \text { se } \lambda_{\Lambda_{1}}\left(\rho_{\Lambda_{1}} \rho_{\Lambda_{2}}^{-1}\right)<\infty \\
1 & \text { caso contrário. }
\end{array}\right.
$$

Então $0<\rho_{\Lambda}<\infty$, e $\rho_{\Lambda}$ é mensurável. Para provar que $\rho_{\Lambda}$ tem as propriedades requeridas seja $\mu \in \mathscr{P}(\Omega, \mathscr{F})$ tal que $\mu \gamma_{\{i\}}=\mu$ para todo $i \in S$. Pela hipótese de indução $\mu=\rho_{\Lambda_{k}}\left(\mu \lambda_{\Lambda_{k}}\right)$. Seja $f \geq 0$ uma função mensurável positiva. Então temos que

$$
\mu \lambda_{\Lambda}(f)=\mu \lambda_{\Lambda_{1} \cup \Lambda_{2}}(f)=\mu \lambda_{\Lambda_{2}}\left(\lambda_{\Lambda_{1}} f\right)=\mu\left(\rho_{\Lambda_{2}}^{-1} \lambda_{\Lambda_{1}} f\right)=\mu \lambda_{\Lambda_{1}}\left(\rho_{\Lambda_{1}} \rho_{\Lambda_{2}}^{-1} \lambda_{\Lambda_{1}} f\right)
$$


usando que $\lambda_{\Lambda_{1}} \circ \lambda_{\Lambda_{1}}=\lambda_{\Lambda_{1}}$ temos que

$$
\begin{aligned}
\mu \lambda_{\Lambda}(f) & =\mu \lambda_{\Lambda_{1}}\left(f \cdot \lambda_{\Lambda_{1}}\left(\rho_{\Lambda_{1}} \rho_{\Lambda_{2}}^{-1}\right)\right)=\mu \lambda_{\Lambda_{1}} \circ \lambda_{\Lambda_{1}}\left(\rho_{\Lambda_{1}} \rho_{\Lambda_{2}}^{-1} \lambda_{\Lambda_{1}} f\right) \\
& =\mu \lambda_{\Lambda_{1}}\left(\lambda_{\Lambda_{1}}(\rho_{\Lambda_{1}} \rho_{\Lambda_{2}}^{-1} \underbrace{\lambda_{\Lambda_{1}} f}_{\mathscr{T}_{\Lambda_{1}} \text { mens. }})\right)=\mu \lambda_{\Lambda_{1}}(\left(\lambda_{\Lambda_{1}} f\right) \underbrace{\lambda_{\Lambda_{1}}\left(\rho_{\Lambda_{1}} \rho_{\Lambda_{2}}^{-1}\right)}_{\mathscr{T}_{\Lambda_{1}} \text { mens. }}) \\
& =\mu \lambda_{\Lambda_{1}}\left(\lambda_{\Lambda_{1}}\left(f \cdot \lambda_{\Lambda_{1}}\left(\rho_{\Lambda_{1}} \rho_{\Lambda_{2}}^{-1}\right)\right)=\mu \lambda_{\Lambda_{1}} \circ \lambda_{\Lambda_{1}}\left(f \cdot \lambda_{\Lambda_{1}}\left(\rho_{\Lambda_{1}} \rho_{\Lambda_{2}}^{-1}\right)\right)\right. \\
& =\mu \lambda_{\Lambda_{1}}\left(f \cdot \lambda_{\Lambda_{1}}\left(\rho_{\Lambda_{1}} \rho_{\Lambda_{2}}^{-1}\right)\right)=\mu\left(f \cdot \rho_{\Lambda_{1}}^{-1} \cdot \lambda_{\Lambda_{1}}\left(\rho_{\Lambda_{1}} \rho_{2}^{-1}\right)\right) \\
& =\mu\left(f \rho_{\Lambda}^{-1}\right) .
\end{aligned}
$$

\section{A.4 EspecificaÇÕES Gibbssianas}

\section{A.4.1 Potenciais}

Definição A.4.1 (Potenciais de interação). Um potencial de interação (ou simplesmente potencial) é uma família de funções $\Phi=\left(\Phi_{A}\right)_{A \in \mathscr{S}}$ de funções $\Phi_{A}: \Omega \rightarrow \mathbb{R}$ com as seguintes propriedades:

(a) Para cada $A \in \mathscr{S}$ a função $\Phi_{A}$ é $\mathscr{F}_{A}$-mensurável;

(b) Para todo $\Lambda \in \mathscr{S}$ e $\omega \in \Omega$ a série

$$
H_{\Lambda}^{\Phi}(\omega)=\sum_{A \in \mathscr{S}, A \cap \Lambda \neq \varnothing} \Phi_{A}(\omega) \quad \text { existe. }
$$

O valor $H_{\Lambda}^{\Phi}(\omega)$ é chamado a energia total de $\omega$ em $\Lambda$ para $\Phi$ e a função é chamada $H_{\Lambda}^{\Phi}$ o Hamiltoniano em $\Lambda$ para $\Phi$. Denote por $h_{\Lambda}^{\Phi}$ o fator de Boltzman definido por

$$
h_{\Lambda}^{\Phi}(\omega)=\exp \left[-H_{\Lambda}^{\Phi}(\omega)\right] .
$$

Proposição A.4.2. Para cada potencial $\Phi$ a família $\left(h_{\Lambda}^{\Phi}\right)_{\Lambda \in \mathscr{S}}$ é o uma pré-modificação positiva.

Demonstração. Sejam $\Lambda \subset \Delta \in \mathscr{S}$ não vazios. Precisamos mostrar que

$$
H_{\Lambda}^{\Phi}(\xi)+H_{\Delta}^{\Phi}(\eta)=H_{\Delta}^{\Phi}(\xi)+H_{\Lambda}^{\Phi}(\eta)
$$

para todos $\xi, \eta \in \Omega \operatorname{com} \xi_{S \backslash \Lambda}=\eta_{S \backslash \Lambda}$, ou equivalentemente, que para todos $\xi, \eta \in \Omega$ temos

$$
H_{\Lambda}^{\Phi}(\xi)-H_{\Delta}^{\Phi}(\xi)=H_{\Lambda}^{\Phi}(\eta)-H_{\Delta}^{\Phi}(\eta) .
$$

Basta provarmos então que $H_{\Lambda}^{\Phi}-H_{\Delta}^{\Phi}$ é $\mathscr{T}_{\Lambda}$ mensurável, o que moralmente significa que $H_{\Lambda}^{\Phi}-H_{\Delta}^{\Phi}$ não depende das coordenadas em $\Omega_{S \backslash \Lambda}$. Mas o que desejamos segue imediatamente da expressão abaixo

$$
H_{\Lambda}^{\Phi}-H_{\Delta}^{\Phi}=\sum_{A \cap \Delta \neq \varnothing, A \cap \Lambda=\varnothing} \Phi_{A}
$$

lembrando que $\mathscr{T}_{\Delta} \subset \mathscr{T}_{\Lambda}$. 
Função de partição. Seja $\lambda \in \mathscr{M}(E, \mathscr{E})$ uma medida a priori fixada. Diremos que um potencial $\Phi$ é $\lambda$-admissivel se

$$
Z_{\Lambda}^{\Phi}(\omega) \equiv \lambda_{\Lambda} h_{\Lambda}^{\Phi}(\omega)=\int \lambda^{\Lambda}(d \xi) \exp \left[-H_{\Lambda}^{\Phi}\left(\xi \omega_{S \backslash \Lambda}\right)\right]
$$

é finito para todo $\Lambda \in \mathscr{S}$ e todo $\omega \in \Omega$. A função $Z_{\Lambda}^{\Phi}(\omega)$ é chamada a função de partição em $\Lambda$ para $\Phi, \omega(\mathrm{e} \lambda)$.

Para cada potêncial $\lambda$-admissível $\Phi$ defina uma família de funções em $\Omega$ do seguinte modo

$$
\rho_{\Lambda}^{\Phi}=h_{\Lambda}^{\Phi} / Z_{\Lambda}^{\Phi}, \quad \Lambda \in \mathscr{S}
$$

segue da Proposição A.3.7 que a família $\rho^{\Phi}=\left(\rho_{\Lambda}^{\Phi}\right)_{\Lambda \in \mathscr{S}}$ é uma $\lambda$-modificação. Como essa observação em mãos temos a seguinte definição fundamental: Distribuição de Gibbs. Seja $\Phi$ um potencial $\lambda$ admissível, $\omega \in \Omega$ e $\Lambda \in \mathscr{S}$. Então a medida de probabilidade

$$
\begin{aligned}
A \rightarrow \gamma_{\Lambda}^{\Phi}(A \mid \omega) & \equiv \rho_{\Lambda}^{\Phi} \lambda_{\Lambda}(A \mid \omega) \\
& =Z_{\Lambda}^{\Phi}(\omega)^{-1} \int \lambda^{\Lambda}(d \xi) \exp \left[-H_{\Lambda}^{\Phi}\left(\xi \omega_{S \backslash \Lambda}\right)\right] 1_{A}\left(\xi \omega_{S \backslash \Lambda}\right)
\end{aligned}
$$

em $(\Omega, \mathscr{F})$ é chamada distribuição de Gibbs em $\Lambda$ com condições de bordo $\omega_{S \backslash \Lambda}$, potencial de iteração $\Phi$ e medida a priori $\lambda$. A $\lambda$-especificação $\gamma^{\Phi}=\left(\gamma_{\Lambda}^{\Phi}\right)_{\Lambda \in \mathscr{S}}=\rho^{\Phi} \lambda$ é chamada de a especificação Gibbssiana para $\Phi$ e $\lambda$. Cada campo aleatório $\mu \in \mathscr{G}(\Phi) \equiv \mathscr{G}\left(\gamma^{\Phi}\right)$ é chamado uma medida de Gibss ou um campo aleatório de Gibss para $\Phi$ e $\lambda$. Em homenagem a Dobrushin, Lanford e Ruelle uma medida de Gibss é frequentemente chamada de um estado DLR. Transição de

Fase. Diremos que um potencial $\Phi$ exibe uma transição de fase de \# $\mathscr{G}(\Phi)>1$.

Os espaços $\mathscr{B}$. Um potencial $\Phi$ é dito absolutamente somável se para todo $i \in S$ temos que

$$
\|\Phi\|_{i} \equiv \sum_{A \in \mathscr{S}, A \ni i}\left\|\Phi_{A}\right\|
$$

é finito, onde $\|\cdot\|$ denota a norma do sup.

a. Esta condição claramente implica a condição (b) de um potencial.

b. Ela sempre implica que $\Phi$ é uniformemente convergente, no sentido de que, para todo $\Lambda \in \mathscr{S}$, a rede $\left(H_{\Lambda, \Delta}^{\Phi}\right)_{\Delta \in \mathscr{S}}$ converge uniformemente para $H_{\Lambda}^{\Phi}$, onde

$$
H_{\Lambda, \Delta}^{\Phi}=\sum_{A \subset \Delta, A \cap \Lambda \neq \varnothing} \Phi_{A} .
$$

Usaremos o símbolo $\mathscr{B}$ para denotar o espaço dos potenciais absolutamente somáveis. As funções $\|\cdot\|_{i}$ em $\mathscr{B}$ são semi-normas e definem uma topologia completamente metrizável e localmente convexa em $\mathscr{B}$, consequentemente $\mathscr{B}$ é um espaço de Frechet. 


\section{A.5 Quasilocalidade}

Vamos descrever quantidades macroscópicas e microscópicas. Diremos que uma função $f: \Omega \rightarrow$ $\mathbb{R}$ descreve um observável macroscópico quando $f$ é mensurável com relação à sigma álgebra caudal

$$
\mathscr{T} \equiv \bigcap_{\Lambda \in \mathscr{S}} \mathscr{F}_{S \backslash \Lambda}
$$

A razão para esta interpretação é óbvia: A $\mathscr{T}$-mensurabilidade de uma função $f$ significa exatamente que o valor de $f$ não é afetado por nenhum comportamento em qualquer região finita de spins. Um olhar ingênuo pode nos levar a acreditar que $\mathscr{T}=\{\varnothing, \Omega\}$. Mas ao contrário $\mathscr{T}$ é muito rica. Um típico exemplo de evento caudal é

$$
\left[\lim _{n \rightarrow \infty}\left|\lambda_{n}\right|^{-1} \sum_{i \in \Lambda_{n}} \sigma_{i} \text { existe e pertence a } B\right]
$$

onde $B \in \mathscr{E}$ e $\left(\Lambda_{n}\right)$ é uma sequência cofinal em $\mathscr{S}$. A condição de "propriedade" na definição de especificação implica que toda especificação $\gamma$ preserva observáveis macroscópicos, no sentido de que $\gamma_{\Lambda} f=f$ sempre que $\Lambda \in \mathscr{S}$ e $f$ limitada $\mathscr{T}$-mensurável. Em particular, $\gamma$ mapeia o conjunto de todas as funções $f \mathscr{T}$-mensuráveis em sí mesmo. Agora queremos tornar preciso o termo "quantidade microscópica". É natural dizer que uma função $f: \Omega \rightarrow \mathbb{R}$ é uma quantidade microscópica se $f$ é arbitrariamente próxima de funções que dependem somente de um número finito de coordenadas. Entretanto não existe um significado canônico do termo "proximo". O significado mais simples é "próximo na norma do sup". Isto nos leva ao conceito de função quasilocal.

Funções locais. Uma função real $f: \Omega \rightarrow \mathbb{R}$ é dita função local se $f$ é $\mathscr{F}_{\Lambda}$-mensurável para algum $\Lambda \in \mathscr{S}$. Para cada $\Lambda \in \mathscr{S}$ escrevemos $\mathscr{L}_{\Lambda}$ para denotar o conjunto de todas as funções limitadas $\mathscr{F}_{\Lambda}$-mensuráveis. Escrevemos $\mathscr{L}=\bigcup_{\Lambda \in \mathscr{S}} \mathscr{L}_{\Lambda}$ para denotar o espaço de todas as funções locais limitadas.

Funções quasilocais. Uma função $f: \Omega \rightarrow \mathbb{R}$ é dita uma função quasilocal se existe uma sequência $\left(f_{n}\right)$ de funções locais tais que $\lim _{n \rightarrow \infty}\left\|f_{n}-f\right\|=0$, onde $\|\cdot\|$ é a norma do sup. Denotaremos por $\overline{\mathscr{L}}$ o conjunto de todas as funções quasilocais limitadas. Note que $\overline{\mathscr{L}}$ é fecho uniforme de $\mathscr{L}$.

\section{Observações:}

a. Uma função mensurável $f$ é quasilocal se e somente se

$$
\lim _{\Lambda \in \mathscr{S}} \sup _{\xi, \eta \in \Omega: \xi_{\Lambda}=\eta_{\Lambda}}|f(\xi)-f(\eta)|=0
$$

Proof. Suponhamos inicialmente que $f$ é quasilocal. Dado $\varepsilon>0$ seja $f_{\Lambda_{n}}$ uma função local $\mathscr{F}_{\Lambda_{n}}$-mensurável tal que $\left\|f_{\Lambda_{n}}-f\right\|<\varepsilon$. Temos da desigualdade triangular que

$$
|f(\xi)-f(\eta)| \leq\left|f(\xi)-f_{\Lambda_{n}}(\xi)\right|+\left|f_{\Lambda_{n}}(\xi)-f_{\Lambda_{n}}(\eta)\right|+\left|f_{\Lambda_{n}}(\eta)-f(\eta)\right|
$$


observe que quando $\xi_{\Lambda_{n}}=\eta_{\Lambda_{n}}$ então a parcela central do lado direito da desigualdade acima é 0 pelo fato de $f_{\Lambda_{n}}$ ser $\mathscr{F}_{\Lambda_{n}}$-mensurável. Portanto temos que

$$
\begin{aligned}
\sup _{\xi, \eta \in \Omega, \xi_{\Lambda}=\eta_{\Lambda_{n}}}|f(\xi)-f(\eta)| & \leq \sup _{\xi, \eta \in \Omega, \xi_{\Lambda}=\eta_{\Lambda_{n}}}\left|f(\xi)-f_{\Lambda_{n}}(\xi)\right|+\sup _{\xi, \eta \in \Omega, \xi_{\Lambda}=\eta_{\Lambda_{n}}}\left|f_{\Lambda_{n}}(\eta)-f(\eta)\right| \\
& <\varepsilon+\varepsilon \\
& =2 \varepsilon .
\end{aligned}
$$

b. Suponha que $E$ seja um espaço métrico e $d$ uma métrica em $\Omega$ que induz a topologia produto. Então cada função $f: \Omega \rightarrow \mathbb{R}$ é quasilocal.

Definição A.5.1. Diremos que uma especificação $\gamma$ é quasilocal quando para cada $\Lambda \in \mathscr{S} e$ $f \in \overline{\mathscr{L}}$ temos que $\gamma_{\Lambda} f \in \overline{\mathscr{L}}$.

Obs: Para ver que uma especificação $\gamma$ é quasilocal é suficiente verificar que $\gamma_{\Lambda} f \in \overline{\mathscr{L}}$ sempre que $f \in \mathscr{L}$ e $\Lambda \in \mathscr{S}$. Também é fácil verificar que a especificação independente é quasilocal.

Proposição A.5.2. Seja $\lambda \in \mathscr{M}(E, \mathscr{E})$ uma medida a priori. A seguintes afirmações são verdade:

(a) Suponha que $\gamma=\rho \lambda$ é uma $\lambda$ especificação. Se cada $\rho_{\Lambda}$ é local, ou se $\lambda$ é finita e cada $\rho_{\Lambda}$ é quase local, então $\gamma$ é quasilocal.

(b) Suponha que $\Phi$ seja um potencial $\lambda$ admissivel tal que todos os hamiltonianos $H^{\Phi}$ são quasilocais. Então $\gamma^{\Phi}$ é quasilocal.

(c) Suponha que E seja finito, $\lambda$ a medida de contagem e $\gamma$ uma especificação quasilocal. Seja $\rho$ a única $\lambda$-modificação tal que $\gamma=\rho \lambda$. Então $\rho_{\Lambda} \in \overline{\mathscr{L}}$ para todo $\Lambda \in \mathscr{S}$.

\section{A.6 O Modelo de Ising: Limite Termodinâmico e For- MALISMO DLR}

\section{A.6.1 Modelo De Ising: Formalismo DLR}

O Modelo de Ising é o arquétipo original das especificações Gibssianas, faremos aqui uma breve introdução sobre ele. Considere $S=\mathbb{Z}^{d}$ e $E=\{0,1\}$ com medida a priori $\lambda=(1 / 2) \delta_{0}+(1 / 2) \delta_{1}$. Considere o potencial $\Phi=\left(\Phi_{A}\right)_{A \in \mathscr{S}}$ onde cada função $\mathscr{F}_{A}$ mensurável $\Phi_{A}$ é dada por

$$
\Phi_{A}(\omega)=\left\{\begin{array}{cc}
-J(i, j) \cdot \omega_{i} \omega_{j} & \text { se } A=\{i, j\} \\
-h(i) \cdot \omega_{i} & \text { se } A=\{i\} \\
0 & \text { caso contrário }
\end{array}\right.
$$

o Hamiltoniano correspondente a volume finito $\Lambda \in \mathscr{S}$, com função de acoplamento $J: \Omega \times \Omega \rightarrow$ $(0, \infty)$ e campo magnético $h: \Omega \rightarrow(0, \infty)$ é dado por

$$
H_{\Lambda}(\omega)=\sum_{A \in \mathscr{S}, A \cap \Lambda \neq \varnothing} \Phi_{A}(\omega)=-\sum_{i \vee j \in \Lambda} J_{i j} \sigma_{i} \sigma_{j}-\sum_{i \in \Lambda} h_{i} \omega_{i} .
$$


Considere então a distribuição de Gibbs associada a esse potencial

$$
\begin{aligned}
A \mapsto \gamma_{\Lambda}^{\Phi}(A \mid \omega) & \equiv \rho_{\Lambda}^{\Phi} \lambda_{\Lambda}(A \mid \omega) \\
& =Z_{\Lambda}^{\Phi}(\omega)^{-1} \int \lambda^{\Lambda}(d \xi) \exp \left[-H_{\Lambda}^{\Phi}\left(\xi \omega_{S \backslash \Lambda}\right)\right] 1_{A}\left(\xi \omega_{S \backslash \Lambda}\right) .
\end{aligned}
$$

Fixada uma condição de contorno $\eta=\omega_{S \backslash \Lambda}$ denote por $\mu_{\Lambda}^{\eta}$ a distribuição de Gibbs descrita acima.

Definição A.6.1. Considere $\Omega=\{0,1\}^{\mathbb{Z}^{d}}$ ordenado linearmente. Dizemos que uma medida $\mu$ em $\Omega_{\Lambda}$ para $\Lambda \subset \mathbb{Z}^{d}$ finito satisfaz satisfaz a desigualdade $F K G$ se para todas funções $f, g$ limitadas $\mathscr{F}_{\Lambda}$ mensuráveis não decrescentes com respeito à ordem em $\Omega_{\Lambda}$ induzida da ordem de $\Omega$ se

$$
\mu(f g) \geq \mu(f) \mu(g) .
$$

Teorema A.6.2 (Desigualdade FKG). Considere o modelo de Ising descrito no inicio desta secção. Então qualquer distribuição de Gibbs a volume finito para esta interação satisfaz a desigualdade FKG.

Demonstração. A prova é feita por indução no tamanho do volume de $\Lambda$. Como já foi observado, quando $|\Lambda|=1$ este fato é trivialmente verdade, isto é,

$$
\mu_{\{x\}}^{\eta}(f g) \geq \mu_{\{x\}}^{\eta}(f) \mu_{\{x\}}^{\eta}(g) .
$$

Agora assuma que a afirmação valha para um subconjunto finito arbitrário $\Lambda \subset \mathbb{Z}^{d}$. Tome $y \in \Lambda^{c}$ e considere $\Lambda^{\prime}=\Lambda \cup\{y\}$. Vamos mostrar que a afirmação é verdadeira para qualquer $\mu_{\Lambda^{\prime}}^{\eta}$ e quaisquer funções $f, g$ não decrescentes $\mathscr{F}_{\Lambda^{\prime}}$ mensuráveis. Note que, pelas equações de compatibilidade temos

$$
\begin{aligned}
\mu_{\Lambda^{\prime}}^{\eta}(f g) & =\int \mu_{\{y\}}^{\eta}(d \xi) \mu_{\Lambda}^{\eta}(f g)=\sum_{\eta_{y}= \pm 1} \mu_{\Lambda}^{\eta}\left(\omega_{y}=\eta_{y}\right) \mu_{\Lambda}^{\eta}(f g) \\
& \geq \sum_{\eta_{y}= \pm 1} \mu_{\Lambda}^{\eta}\left(\omega_{y}=\eta_{y}\right) \mu_{\Lambda}^{\eta}(f) \mu_{\Lambda}^{\eta}(g)
\end{aligned}
$$

onde usamos a hipótese de indução. Visto que a soma sobre $\eta_{y}$ satisfaz a desigualdade $F K G$ trivialmente, precisamos apenas mostrar que $\mu_{\Lambda}^{\eta}(f)$ é uma função monótona da variável $\eta_{y}$ quando $f$ é monótona. Suprimindo todas as variáveis com exceção de $\eta_{y}$ esta tarefa se reduz a mostrar que $\mu_{\Lambda}^{+1}(f(+1)) \geq \mu_{\Lambda}^{-1}(f(-1))$, vamos mostrar a afirmação mais forte $\mu_{\Lambda}^{+1}(f(+1)) \geq$ $\mu_{\Lambda}^{-1}(f(+1))$. Relembrando que $\eta_{y}$ pode ser visto considerado como uma variável real temos que

$$
\frac{d}{d \eta_{y}} \mu_{\Lambda}^{\eta_{y}}(f(+1))=\mu_{\Lambda}^{\eta_{y}}\left(f(+1) \sum_{z \in \Lambda} J_{z y} \sigma_{y}\right)-\mu_{\Lambda}^{\eta_{y}}\left(\sum_{z \in \Lambda} J_{z y} \sigma_{y}\right) \mu_{\Lambda}^{\eta_{y}}(f(+1)) \geq 0
$$

onde a primeira igualdade provém da diferenciação e a segunda desigualdade vale pois $\sum_{z \in \Lambda} J_{z y} \sigma_{y}$ é uma função não decrescente visto que todos os $J_{x y}$ são positivos, terminando o argumento. 


\section{A.6.2 Modelo de Isign: Limite Termodinâmico}

No capítulo 1 as medidas de Gibbs eram para nós medidas em conjuntos $\Omega_{\Lambda}$ finito. Nesta secção queremos estudar o modelo de Ising em todo o latice $\mathbb{Z}$. As medidas de Gibbs serão probabilidades definidas no espaço de configurações $\Omega=\{-1,1\}^{\mathbb{Z}}$. Estas medias serão obtidas através de limites fracos quando $\Lambda \uparrow \mathbb{Z}$. Como sempre, estamos considerando em $\{-1,1\}$ a topologia discreta e em $\Omega$ a topologia produto. Segue do Teorema de Tychonov que $\Omega$ é compacto. Naturalmente, trabalharemos em $\Omega$ com a sigma álgebra de Borel $\mathscr{B}(\Omega)$. Denotaremos por $\Omega_{\Lambda}$ o conjunto $\pi_{\Lambda}^{-1}\left(\{-1,1\}^{\Lambda}\right)$. E consideraremos em $\Omega_{\Lambda}$ a sigma álgebra induzida pela projeção $\pi_{\Lambda} \mathrm{e}$ pela sigma álgebra em $\{-1,1\}^{\Lambda}$, a qual denotaremos por $\mathscr{B}_{\Lambda}(\Omega)$.

A partir de agora vamos assumir que $J=\left(J_{i j}\right)$ é uma interação ferromagnética somável, i.e, satisfaz $\sup _{i \in \mathbb{Z}} \sum_{j \in \mathbb{Z}} J_{i j}<\infty$. Naturalmente, queremos definir Hamiltonianos $H_{\Lambda}$ a "volume finito", como foi definido no capítulo 1 . De fato as definições são bastante próximas, com efeito os $\Omega_{\Lambda}$ definidos no capítulo 1 e nesta secção são moralmente o mesmo conjunto, pois qualquer função $f: \Omega \rightarrow \mathbb{R}$ mensurável com relação à sigma álgebra $\mathscr{B}_{\Lambda}(\Omega)$ não depende das coordenadas fora de $\Omega_{\Lambda}$. A principal diferença entre os dois contextos é que no presente contexto, ao escolhermos uma configuração em $\Omega_{\Lambda}$ necessariamente precisamos escolher uma configuração em $\Omega_{\Lambda^{c}}$, a qual será chamada de condição de contorno.

Para definir os Hamiltonianos a volume finito, vamos considerar apenas os intervalos simétricos de inteiros $\Lambda=[-m, m] \cap \mathbb{Z}$. Definiremos $H_{\Lambda}: \Omega \rightarrow \mathbb{R}$ de modo muito próximo ao capítulo 1 , com a diferença de que passaremos a levar em conta no Hamiltoniano as interações dos spins em $\Lambda$ com os spins em $\Lambda^{c}$. Então nosso procedimento será, escoher uma condição de contorno $\eta$ e definir

$$
H_{\Lambda, h}^{\eta}(\omega)=\sum_{i \in \Lambda}\left\{\sum_{j \in \Lambda} J_{i j} \omega_{i} \omega_{j}-h_{i} \omega_{i}+\sum_{j \in \Lambda^{c}} J_{i j} \eta_{j} \omega_{i}\right\} .
$$

A medida de Gibbs a volume $\Lambda$ e condição de contorno $\eta$ é definida como sendo a medida de probabilidade $\mu_{\Lambda, \beta, h}^{\eta}$ definida em $\mathscr{B}_{\Lambda}(\Omega)$ que associa a cada $\omega \in \Omega_{\Lambda}$ a seguinte probabilidade:

$$
\mu_{\Lambda, \beta, h}^{\eta}(\{\omega\})=\frac{\exp \left[-\beta H_{\Lambda, h}^{\eta}(\omega)\right]}{Z_{\Lambda, \beta, h}^{\eta}}
$$

onde $Z_{\Lambda, \beta, h}^{\eta}(\omega)$ é um fator de normalização chamado função de partição, definido por

$$
Z_{\Lambda, \beta, h}^{\eta}=\sum_{\substack{\omega \in \Omega_{\Lambda} ; \omega_{i}=\eta_{i} \\-n \leq i \leq n}} \exp \left[-\beta H_{\Lambda, h}^{\eta}(\omega)\right] .
$$

Temos duas condições de contorno particularmente especiais que são quando $\eta_{1}=(1, \ldots, 1 \ldots)$ e quando $\eta_{2}=(-1, \ldots,-1, \ldots)$. Nestes dois casos as medidas de Gibbs com condição de contorno $\eta_{1}$ e $\eta_{2}$ dois são denotadas de maneira diferente, são denotadas por $\mu_{\Lambda, \beta, h}^{+}$e $\mu_{\Lambda, \beta, h}^{-}$ respectivamente.

\section{O Limite TermodinÂMico}

Podemos pensar em cada medida de Gibbs $\mu_{\Lambda, \beta, h}^{\eta}$ em $\mathcal{M}\left(\Omega_{\Lambda}\right)$ como sendo uma medida em $\mathcal{M}(\Omega)$. De fato podemos estender $\mu_{\Lambda, \beta, h}^{\eta}$ naturalmente à medida $\bar{\mu}_{\Lambda, \beta, h}^{\eta}$ em $\mathcal{M}(\Omega)$ definindo-se 
para cada $A \in \Omega$

$$
\bar{\mu}_{\Lambda, \beta, h}^{\eta}(A)=\mu_{\Lambda, \beta, h}^{\eta}\left(1_{A \cap\left[\omega \in \Omega ; \omega_{i}=\eta_{i}, \forall i \in \Lambda^{c}\right]}\right) .
$$

Por abuso de notação denotaremos a extensão $\bar{\mu}_{\Lambda, \beta, h}^{\eta}$ pelo mesmo símbolo $\mu_{\Lambda, \beta, h}^{\eta}$. Agora considere o conjunto dos pontos de acumulação, na topologia fraca, das medidas de Gibbs definidas acima

$$
\mathscr{G}_{\beta, h}^{0}=\left\{\mu \in \mathcal{M}(\Omega) ; \mu=w-\lim _{n_{i} \uparrow \infty} \mu_{\Lambda_{n_{i}}, \beta, h}^{\eta}, \eta \in \Omega\right\}
$$

onde $\left\{\Lambda_{n_{i}}\right\}_{n_{i} \in \mathbb{Z}}$ é uma sequência crescentes de intervalos simétricos em $\mathbb{Z}$ e $\eta$ é uma condição de contorno qualquer. Pela compacidade do espaço $\mathscr{M}(\Omega)$ segue que o conjunto $\mathscr{G}_{\beta, h}^{0}$ é não vazio. Finalmente definimos $\mathscr{G}_{\beta, h}$ como sendo o fecho da envoltória convexo de $\mathscr{G}_{\beta, h}^{0}$. Qualquer medida de probabilidade em $\mathscr{G}_{\beta, h}^{0}$ é dita uma medida de Gibbs a volume infinito.

\section{Pressão MangetizaÇão E Energia Livre}

Assim como no capítulo 1 temos conceitos análogos de pressão, magnetização e energia live. Para cada $\Lambda$ intervalo simétrico finito, definimos as quantidades $\Psi_{\Lambda, \beta, h}^{\eta}$ e $P_{\Lambda, \beta, h}^{\eta}$ como segue

$$
\Psi_{\Lambda, \beta, h}^{\eta}=-\frac{1}{\beta} \frac{\ln Z_{\Lambda, \beta, h}^{\eta}}{|\Lambda|}, P_{\Lambda, \beta, h}=\frac{\ln Z_{\Lambda, \beta, h}^{\eta}}{|\Lambda|}
$$

são chamadas respectivamente de energia livre e pressão a volume finito. Considere a variável aleatória que em cada configuração $\omega \in \Omega_{\Lambda}$ é dada pela soma finita $S_{\Lambda}(\omega)=\sum_{i \in \Lambda} \omega_{i}$. Definimos a magnetização a volume finito $\Lambda$ com condição de contorno $\eta$ como sendo a quantidade

$$
M_{\Lambda, \beta, h}^{\eta}=\int_{\Omega_{\Lambda}} S_{\Lambda}(\omega) d \mu_{\Lambda, \beta, h}^{\eta}
$$

Temos então o seguinte resultado:

Proposição A.6.3. Os seguintes limites abaixo sempre existem

$$
\psi(h, \beta)=\lim _{\Lambda \uparrow \mathbb{Z}}-\frac{1}{\beta} \frac{\ln Z_{\Lambda, \beta, h}^{\eta}}{|\Lambda|}, P(h, \beta)=\lim _{\Lambda \uparrow \mathbb{Z}} \frac{\ln Z_{\Lambda, \beta, h}^{\eta}}{|\Lambda|} \text { e } m(\beta, h)=\lim _{\Lambda \uparrow \mathbb{Z}} \frac{M_{\Lambda, \beta, h}^{\eta}}{|\Lambda|}
$$

independem da condição de contorno $\eta$ e além disso temos a seguintes relações:

$$
m(\beta, h)=-\frac{\partial \psi(\beta, h)}{\partial h}
$$

para cada $\beta>0$ fixado, $m(\beta, 0)=0$ e

$$
m(\beta,+)=-\frac{\partial \psi(\beta, 0)}{\partial h^{+}} \geq 0 \geq m(\beta,-)=-\frac{\partial \psi(\beta, 0)}{.} \partial h^{-}
$$

Demonstração. Ver [15] página 114, Lema IV.6.4 e página 105 Teorema IV.5.2. 
Seja $J=\left(J_{i j}\right)$ uma interação ferromagnética somável em $\mathbb{Z}$. E consideremos o modelo de Ising proveniente desta interação para algum campo magnético constante de intensidade $h$, então defina o inverso da temperatura crítica como sendo a quantidade

$$
\beta_{c}=\sup \{\beta>0: m(\beta,+)=0\},
$$

que pode ser finita ou infinita.

Proposição A.6.4. Seja $J=\left(J_{i j}\right)$ uma interação ferromagnética invariante por translação somável em $\mathbb{Z}$. Se $\sum_{k \in \mathbb{Z}}|k| J(k)<\infty$ então $\beta_{c}=+\infty$. Além disso $\psi(\beta, h)$ é diferenciável em $h=0$ e $m(\beta,+)=0=m(\beta,-)$.

Demonstração. Ver [15] página 106 Teorema IV.5.3.

Proposição A.6.5. Seja $J=\left(J_{i j}\right)$ uma interação ferromagnética invariante por translação somável em $\mathbb{Z}$ e suponha que, $\sum_{k \in \mathbb{Z}}|k| J(k)<\infty$. Então as seguinte afirmaçôes são verdadeiras

(a) Para cada $\beta>0$ os limite fracos $\mu_{\beta, h}^{+}=w$ - $\lim _{\Lambda \uparrow \mathbb{Z}} \mu_{\Lambda, \beta, h}^{+}$e $\mu_{\beta, h}^{-}=w-\lim _{\Lambda \uparrow \mathbb{Z}} \mu_{\Lambda, \beta, h}^{-}$existem e coincidem, i.e, $\mu_{\beta, h}^{-}=\mu_{\beta, h}^{+}$. Neste caso denotamos este limite fraco por $\mu_{\beta, h}$.

(b) O limite fraco $\mu_{\beta, h}$ é invariante por translação, i.e, pelo shift. Além disso é o único elemento de $\mathscr{G}_{\beta, h}$.

(c) Em particular, quando $h=0$ temos que $\int \omega_{0} d \mu_{\beta, 0}=m(\beta, 0)=0$.

Demonstração. Este teorema é um corolário da proposição anterior e do Teorema IV.6.5 na página 115 de [15].

\section{A.6.3 Desigualdades GKS-I E GKS-II Para o MOdelo De Ising}

Definição A.6.6. Dado um subconjunto $A \subset \Lambda$, considere a função $\sigma_{A}: \Omega_{\Lambda} \rightarrow\{-1,1\}$ definida por $\sigma_{A}(\omega)=\prod_{i \in A} \omega_{i}$.

Teorema A.6.7 (Desigualdade GKS-I). Seja $\Lambda \subset \mathbb{Z}$ finito e considere o modelo de ising no volume $\Lambda$ com hamiltoniano

$$
H_{\Lambda}(\omega)=-\sum_{i \in \Lambda}\left\{\sum_{j \in \Lambda} J_{i j} \omega_{i} \omega_{j}+h_{i} \omega_{i}\right\}
$$

onde $J_{i j} \geq 0$ e $h_{i} \geq 0$. Então para qualquer subconjunto não vazio $A \subset \Omega$, temos que

$$
\mu_{\Lambda}\left(\sigma_{A}\right) \geq 0
$$

Demonstração. Como a função de partição é uma soma de parcelas positivas, ou seja, $Z_{\Lambda}>0$, basta mostrar que

$$
\sum_{\omega \in \Omega_{\Lambda}} \sigma_{A}(\omega) \exp \left[-H_{\Lambda}(\omega)\right] \geq 0
$$


Concentremonos no fator de Boltzman:

$$
\exp \left[-H_{\Lambda}(\omega)\right]=\prod_{i \in \Lambda} e^{h_{i} \omega_{i}} \prod_{j \in \Lambda} \exp \left[J_{i j} \sigma_{i} \sigma_{j}\right]
$$

expandindo em série e Taylor obtemos

$$
\exp \left[J_{i j} \omega_{i} \omega_{j}\right]=1+\frac{J_{i j} \omega_{i} \omega_{j}}{1 !}+\frac{\left(J_{i j} \omega_{i} \omega_{j}\right)^{2}}{2 !}+\frac{\left(J_{i j} \omega_{i} \omega_{j}\right)^{3}}{3 !}+\cdots
$$

Agora observe que como $\omega_{i}= \pm 1, \forall i \in \Lambda$ temos que

$$
\left(J_{i j} \omega_{i} \omega_{j}\right)^{k}=\left\{\begin{array}{cc}
J_{i j}^{k} & \text { se } k \text { é par } \\
J_{i j}^{k} \omega_{i} \omega_{j} & \text { se } k \text { é impar. }
\end{array}\right.
$$

Agrupando os termos de expoentes pares separadamente dos termos de expoentes impares obtemos

$$
\begin{aligned}
\exp \left[J_{i j} \omega_{i} \omega_{j}\right] & =\cosh \left(J_{i j}\right)+\sinh \left(J_{i j}\right) \omega_{i} \omega_{j} \\
& =\cosh \left(J_{i j}\right)\left[1+\tanh \left(J_{i j} \omega_{i} \omega_{j}\right)\right]
\end{aligned}
$$

Substituindo A.20 em A.18 obtemos a seguinte expressão para o fator de Boltzman

$$
\exp \left[-H_{\Lambda}(\omega)\right]=\left\{\prod_{j \in \Lambda} \cosh \left(J_{i j}\right)\left[1+\tanh \left(J_{i j} \omega_{i} \omega_{j}\right)\right]\right\} \cdot \prod_{i \in \Lambda} e^{h_{i} \sigma_{i}}
$$

Portanto substituindo A.22 em A.17 obtemos

$$
\sum_{\omega \in \Omega_{\Lambda}} \sigma_{A}(\omega) \exp \left[-H_{\Lambda}(\omega)\right]=\sum_{\omega \in \Omega_{\Lambda}} \prod_{i \in \Lambda} e^{h_{i} \omega_{i}} \prod_{j \in \Lambda} \cosh \left(J_{i j}\right)\left[\sigma_{A}+\tanh \left(J_{i j}\right) \omega_{i} \omega_{j} \sigma_{A}\right]
$$

Desenvolvendo inicialmente os produtórios da expressão acima obtemos uma soma $\sum_{m} c_{m} P_{m}$ na qual cada termo é constituído de um produto envolvendo as variáveis de spin

$$
P_{m}=\omega_{i_{1}}^{n_{i_{1}}} \cdots \omega_{i_{m}}^{n_{i_{m}}} \prod_{i \in \Lambda} e^{h_{i} \omega_{i}}
$$

e de um prefator da forma $c_{m}$ envolvendo produtos de funções hiperbólicas, ora $\cosh \left(J_{i j}\right)$, ora $\cosh \left(J_{i j}\right) \tanh \left(J_{i j}\right)$. Sendo assim

$$
\sum_{\omega \in \Omega_{\Lambda}} \sigma_{A}(\omega) \exp \left[-H_{\Lambda}(\omega)\right]=\sum_{\omega \in \Omega_{\Lambda}} \sum_{m} c_{m} P_{m}=\sum_{m} c_{m} \sum_{\omega \in \Omega_{\Lambda}} P_{m}
$$

Observe que cada $c_{m} \geq 0$, pois $\cosh \left(J_{i j}\right)$ e $\tanh \left(J_{i j}\right)$ o são para $J_{i j} \geq 0, \forall i, j \in \Lambda$. Por outro lado caso todos os expoentes $n_{i}$ que aparecem na expressão de $P_{m}$ sejam pares teremos que $P_{m}=\prod_{i \in \Lambda} e^{h_{i} \omega_{i}}$ e por conseguinte a soma das configurações é positiva, pois

$$
\sum_{\omega \in \Omega_{\Lambda}} \prod_{i \in \Lambda} e^{h_{i} \omega_{i}}>0
$$


Entretanto, caso ocorra a existência de pelo menos um $n_{j}$ impar em $P_{m}$, teremos que $P_{m}=$ $\omega_{j}^{n_{j}} \prod_{i \in \Lambda} e^{h_{i} \omega_{i}}$ e por conseguinte a soma das configurações é positiva pois para $h_{j}$ não negativo temos

$$
\sum_{\omega_{j}= \pm 1} \omega_{j} e^{h_{j} \omega_{j}}=2 \sinh \left(h_{j}\right) .
$$

Segue daí.

Teorema A.6.8 (Desigualdade GKS-II). Seja $\Lambda \subset \mathbb{Z}$ finito e considere o modelo de ising no volume $\Lambda$ com hamiltoniano

$$
H_{\Lambda}(\omega)=-\sum_{i \in \Lambda}\left\{\sum_{j \in \Lambda} J_{i j} \omega_{i} \omega_{j}+h_{i} \omega_{i}\right\}
$$

onde $J_{i j} \geq 0$ e $h_{i} \geq 0$. Então para quaisquer $A, B \subset \Lambda$ subconjuntos não vazios temos que

$$
\mu_{\Lambda}\left(\sigma_{A} \sigma_{B}\right)-\mu_{\Lambda}\left(\sigma_{A}\right) \mu_{\Lambda}\left(\sigma_{B}\right) \geq 0 .
$$

Demonstração. Considere o novo sistema definido em $\Omega_{\Lambda} \times \Omega_{\Lambda}$ cujo Hamiltoniano associado $H_{2}: \Omega_{\Lambda} \times \Omega_{\Lambda} \rightarrow \mathbb{R}$ é dado por $H_{2}(\omega, \tilde{\omega})=H_{\Lambda}(\omega)+H_{\Lambda}(\tilde{\omega})$. Considere ainda as funções $\omega_{A}, \tilde{\omega}_{A}$ : $\Omega_{\Lambda} \times \Omega_{\Lambda} \rightarrow\{-1,1\}$ Tais que $\omega_{A}(\omega, \tilde{\omega})=\sigma_{A}(\omega)$ e $\tilde{\omega}_{A}(\omega, \tilde{\omega})=\sigma_{A}(\tilde{\omega})$. Denote por $\mu_{\Lambda}^{\text {prod }}$ a medida de Gibbs associada ao Hamiltoniano $H_{2}$. Como o estabelecido acima temos uma reformulação da desigualdade desejada, mais precisamente, basta provar que $\mu_{\Lambda}^{\text {prod }}\left(\omega_{A} \omega_{B}-\omega_{A} \tilde{\omega}_{B}\right) \geq 0$. De fato, temos

$$
\begin{aligned}
\mu_{\Lambda}\left(\sigma_{A} \sigma_{B}\right)-\mu_{\Lambda}\left(\sigma_{A}\right) \mu_{\Lambda}\left(\sigma_{B}\right) & =\mu_{\Lambda}^{\text {prod }}\left(\omega_{A} \omega_{B}\right)-\mu_{\Lambda}^{\text {prod }}\left(\omega_{A}\right) \mu_{\Lambda}^{\text {prod }}\left(\tilde{\omega}_{B}\right) \\
& =\mu_{\Lambda}^{\text {prod }}\left(\omega_{A} \omega_{B}-\omega_{A} \tilde{\omega}_{B}\right)
\end{aligned}
$$

onde a primeira igualdade provém de que pela definição de $\omega_{A}$, temos $\mu_{\Lambda}^{\text {prod }}\left(\omega_{A}\right)=\mu_{\Lambda}\left(\sigma_{A}\right)$ e a segunda igualdade decorre da igualdade $\mu_{\Lambda}^{\text {prod }}\left(\omega_{A} \tilde{\omega}_{B}\right)=\mu_{\Lambda}^{\text {prod }}\left(\omega_{A}\right) \mu_{\Lambda}^{\text {prod }}\left(\tilde{\omega}_{B}\right)$. Defina para cada $i \in \Lambda$ as funções $X_{i}, Y_{i}: \Omega_{\Lambda} \times \Omega_{\Lambda} \rightarrow\{-1,0,1\}$ pondo $X_{i}(\omega, \tilde{\omega})=\left(\omega_{i}-\tilde{\omega}_{i}\right) / \sqrt{2}$ e $Y_{i}(\omega, \tilde{\omega})=\left(\omega_{i}+\tilde{\omega}_{i}\right) / \sqrt{2}$. Podemos reescrever A.23 em termos de $X_{i}, Y_{i}$ do seguinte modo

$$
\begin{aligned}
\mu_{\Lambda}\left(\sigma_{A} \sigma_{B}\right)-\mu_{\Lambda}\left(\sigma_{A}\right) \mu_{\Lambda}\left(\sigma_{B}\right) & =\mu_{\Lambda}^{\mathrm{prod}}\left(\omega_{A} \omega_{B}-\omega_{A} \tilde{\omega}_{B}\right) \\
& =\mu_{\Lambda}^{\mathrm{prod}}\left(\prod_{i \in A} \frac{X_{i}+Y_{i}}{\sqrt{2}}\left[\prod_{i \in B} \frac{X_{i}+Y_{i}}{\sqrt{2}}-\prod_{i \in B} \frac{X_{i}-Y_{i}}{\sqrt{2}}\right]\right)
\end{aligned}
$$

expandindo os produtórios entre colchetes, todo termo com coeficiente negativo da expansão do produtório à direita terá um equivalente positivo no produtório à esquerda. Esses termos se cancelarão restando apenas termos positivos de produtórios de $X_{i}, Y_{j} \operatorname{com} i, j \in B$. Então ao expandirmos toda a expressão acima teremos uma soma de termos da forma

$$
\mu_{\Lambda}^{\mathrm{prod}}\left(\prod_{i \in C} X_{i} \prod_{j \in D} Y_{j}\right)
$$


sendo assim se provarmos que qualquer termo da forma A.27 é positivo a demonstração estará provada. Ponhamos $X_{A}=\prod_{i \in A} X_{i} Y_{B}=\prod_{i \in B} Y_{i}$, precisamos demonstrar então que $\mu_{\Lambda}^{\text {prod }}\left(X_{A} Y_{A}\right) \geq 0$. Por definição temos que

$$
\mu_{\Lambda}^{\operatorname{prod}}\left(X_{A} Y_{A}\right)=\sum_{\omega, \tilde{\omega} \in \Omega_{\Lambda}} X_{A}(\omega, \tilde{\omega}) Y_{B}(\omega, \tilde{\omega}) \frac{\exp \left[-H_{2}(\omega, \tilde{\omega})\right]}{Z_{2}},
$$

note que, como $Z_{2}$, a função de partição, é sempre positiva, basta estudarmos a expressão $\sum_{\omega, \tilde{\omega} \in \Omega_{\Lambda}} X_{A}(\omega, \tilde{\omega}) Y_{B}(\omega, \tilde{\omega}) \exp \left[-H_{2}(\omega, \tilde{\omega})\right]$. Temos que

$$
\begin{aligned}
H_{2}(\omega, \tilde{\omega}) & =H_{\Lambda}(\omega)+H_{\Lambda}(\tilde{\omega})=-\sum_{i \in \Lambda}\left\{\sum_{j \in \Lambda} J_{i j} \omega_{i} \omega_{j}+h_{i} \omega_{i}+\sum_{j \in \Lambda} J_{i j} \tilde{\omega}_{i} \tilde{\omega}_{j}+h_{i} \tilde{\omega}_{i}\right\} \\
& =-\sum_{i \in \Lambda}\left\{\sum_{i \in \Lambda} 2 J_{i j}\left[\omega_{i} \omega_{j}+\tilde{\omega}_{i} \tilde{\omega}_{j}\right]+h_{i}\left[\omega_{i}+\tilde{\omega}_{i}\right]\right\} \\
& =-\sum_{i \in \Lambda}\left\{\sum_{j \in \Lambda} 2 J_{i j}\left[X_{i}(\omega, \tilde{\omega}) X_{j}(\omega, \tilde{\omega})+Y_{i}(\omega, \tilde{\omega}) Y_{j}(\omega, \tilde{\omega})\right]+h_{i} \sqrt{2} Y_{i}(\omega, \tilde{\omega} .\} .\right.
\end{aligned}
$$

Omitindo a dependência em $\omega$ e $\tilde{\omega}$ temos a expressão mais enxuta para o Hamiltoniano $H_{2}$

$$
H_{2}=-\sum_{i \in \Lambda}\left\{J_{i j}\left[X_{i} X_{j}+Y_{i} Y_{j}\right]+h_{i} \sqrt{2} Y_{i}\right\} .
$$

Substituindo a expressão acima em $\exp \left[-H_{2}(\omega, \tilde{\omega})\right]$, cancelando os sinais de de menos e fazendo a expansão da exponencial em serie de Taylor, observamos que $\mu_{\Lambda}^{\text {prod }}\left(X_{A} Y_{A}\right)$ se decompõe em um somatório de termos da forma $X_{A} Y_{B}\left(H_{2}\right)^{n}$. Como o somatório inicial é tomado $\omega, \tilde{\omega} \in \Omega_{\Lambda}$ podemos alterar a ordem do somatório, deixando em evidência os termos dependentes apenas de $\omega_{i}, \tilde{\omega}_{i}$. O que sobra é produto de vários somatórios da forma

$$
\sum_{\omega_{i}, \tilde{\omega}_{i} \in\{+,-1\}} X_{i}^{m}(\omega, \tilde{\omega}) Y_{i}^{n}(\omega, \tilde{\omega})
$$

sendo $m, n$ dois números inteiros não negativos. Assim, basta provar que

$$
\sum_{\omega_{i}, \tilde{\omega}_{i} \in\{+,-1\}} X_{i}^{m}(\omega, \tilde{\omega}) Y_{i}^{n}(\omega, \tilde{\omega})=\sum_{\omega_{i}, \tilde{\omega}_{i} \in\{+,-1\}}\left(\frac{\omega_{i}-\tilde{\omega}_{i}}{\sqrt{2}}\right)^{m}\left(\frac{\omega_{i}+\tilde{\omega}_{i}}{\sqrt{2}}\right)^{n} \geq 0 .
$$

Se $m, n$ forem pares a desigualdade acima é trivialmente verdade. Caso contrário, $m$ for ímpar, os termos com $\omega_{i}=\tilde{\omega}_{i}$ são nulos, restando apenas, os termos com $\omega \neq \tilde{\omega}_{i}$, mas neste caso $\omega_{i}+\tilde{\omega}_{i}=0$, forçando que o somatório acima seja nulo. O caso $n$ impar é análogo, provando então o desejado.

Observação A.6.9. Uma inspeção nas provas de GKS-I e GKS II, mostra que as desigualdades de correlação são válidas também para sistemas com Hamiltoniano do tipo

$$
H_{\Lambda_{n}, k}(\omega)=-\sum_{i \in \Lambda_{n}}\left\{\sum_{j \in \Lambda_{n+k}} J_{i j} \omega_{i} \omega_{j}+h_{i} \omega_{i}\right\}
$$


Ou seja, se $\mu_{\Lambda_{n}, k}$ é a medida de Gibbs associada ao Hamiltoniano acima, temos que é verdadeira a desigualdade abaixo

$$
\mu_{\Lambda_{n}, k}\left(\sigma_{A} \sigma_{B}\right)-\mu_{\Lambda_{n}, k}\left(\sigma_{A}\right) \mu_{\Lambda_{n}, k}\left(\sigma_{B}\right) \geq 0
$$

Suponha que tenhamos a hipótese adicional de que as constantes de acoplamento $\left(J_{i j}\right)_{i, j \in \mathbb{Z}}$ além de serem não-negativas formam uma sequência tal que $\sup _{i \in \mathbb{Z}} \sum_{j \in \mathbb{Z}}\left|J_{i j}\right|<\infty$. Então as desigualdades GKS-I e GKS-II valem para limites termodinâmicos de medidas de Gibbs com o Hamiltoniano $H_{n}$ dado por

$$
H_{n}(\omega)=\sum_{i=-n}^{n}\left\{\sum_{j \in \mathbb{Z}} J_{i j} \omega_{i} \omega_{j}+h_{i} \omega_{i}\right\}
$$

e condições de contorno positiva e livre. 


\section{Apêndice B}

\section{Alguns Resultados Análise E TEORIA ESPECTRAL}

\section{B.1 Analiticidade em Espaços de Banach}

Definição B.1.1. Sejam $\left(X,\|\cdot\|_{X}\right)$ e $\left(Y,\|\cdot\|_{Y}\right)$ espaços de Banach e $U$ um subconjunto aberto de $X$. Para cada $k \in \mathbb{N}$, a função $F: U \rightarrow Y$ é dita $k$-diferenciável em $x$ se para cada $j \in\{1, \ldots, k\}$, existe a transformação j-linear limitada $D^{j} F(x): X^{j} \rightarrow Y$ tal que

$$
D^{j-1} F\left(x+v_{j}\right)\left(v_{1}, \ldots, v_{j-1}\right)-D^{j-1} F(x)\left(v_{1}, \ldots, v_{j-1}\right)=D^{j} F(x)\left(v_{1}, \ldots, v_{j}\right)+o_{j}\left(v_{j}\right)
$$

on de $o_{j}: X \rightarrow Y$ é tal que $\lim _{v \rightarrow 0}\left\|o_{j}(v)\right\|_{Y} /\left(\|v\|_{X}\right)=0$.

Dizemos que $F$ tem derivadas de todas as ordens em $U$, se para qualquer $k \in \mathbb{N}$, e qualquer $x \in U, F$ é $k$-diferenciável em $x$.

Definição B.1.2. Sejam $X$ e $Y$ espaços de Banach e $U$ um subconjunto aberto de $X$. Uma função $F: U \rightarrow Y$ é chamada analytica $U$, quando $F$ tem derivadas de todas as ordens em $U$, e para cada $x \in U$ existe uma vizinhança aberta $U_{x}$ de $x$ em $U$ tal que para todos $v \in U_{x}$, temos que

$$
F(x+v)-F(x)=\sum_{j=1}^{\infty} \frac{1}{n !} D^{j} F(x) v^{j}
$$

onde $D^{j} F(x) v^{j}=D^{j} F(x)(v, \ldots, v)$ e $D^{j} F(x)$ é j-esima derivada de $F$ em $x$.

Se $F: U \rightarrow Y$ é analitica em $U$, então para cada $n \in \mathbb{N}$, a expansão de Taylor de ordem $n$ é

$$
F(x+v)=F(x)+D^{1} F(x) v+\frac{D^{2} F(x) v^{2}}{2}+\frac{D^{3} F(x) v^{3}}{6}+\ldots+\frac{D^{n} F(x) v^{n}}{n !}+o_{n+1}(v)
$$

onde $o_{n+1}(v)=\sum_{j=n+1}^{\infty}(1 / n !) D^{j} F(x) v^{j}$ satisfaz $\lim _{v \rightarrow 0}\left\|o_{n+1}(v)\right\|_{Y} /\|v\|_{X}^{n}=0$. 


\section{B.2 Alguns Resultados de AnÁlise}

Nesta seção vamos enunciar alguns importantes teoremas de Análise que foram usados ao longo do texto.

Teorema B.2.1 (Teorema do ponto fixo de Schauder-Tychonov). Seja E um espaço de Banach e $F: E \rightarrow E$ um mapa contínuo. Seja $C \subset E$ um conjunto compacto, convexo e não vazio. Se $F(C) \subset C$ então $F$ tem pelo menos um ponto fixo em $C$.

O seguinte resultado é uma das poucas ferramentas concretas que se possui quando se deseja invstigar compacidade no espaço $C(X, \mathbb{R})$ com $X$ compacto.

Teorema B.2.2 (Arzelà-Àscoli). Seja $E \subset C(X, \mathbb{R})$ um subconjunto do espaço das funções continuas tomando valores em $\mathbb{R}$, onde $X$ é um espaço métrico compacto. A fim de que $E$ seja relativamente compacto na norma da convergência uniforme em $C(X, \mathbb{R})$ é necessário e suficiente que

(a) E seja equicontínu;

(b) para cada $x \in X$ o conjunto $E(x)=\{f(x) ; f \in E\}$ seja limitado.

Existem várias versões do Teorema de Riez Markov em diferentes contextos neste texto usamos em alguns momentos a versão enunciada abaixo.

Teorema B.2.3 (Teorema da Representação de Riez-Markov). Seja X um espaço topológico compacto e de Hausdorff. Para cada funcional linear positivo $\psi$ definido sobre $C(X)$, existe uma única medida sinalada finita $\mu$ sobre os Borelianos de $X$ tal que

$$
\psi(f)=\int_{X} f d \mu \quad \forall f \in C(X)
$$

\section{B.3 Elementos de TeOria Espectral}

Nesta seção vamos enumerar alguns resultados clássicos de Teoria Espectral básica, para mais detalhes sugerimos [33]. Seja $X$ um espaço de Banach e $T: X \rightarrow X$ um operadoe linear, definimos o espectro do operador $T$ por

$$
\operatorname{spec}(T)=\left\{\lambda \in \mathbb{C} ;(\lambda I-T)^{-1} \text { não existe }\right\} .
$$

O conjunto resolvente $\rho(T)$ de $T$ é definido como o complemento do espectro $\operatorname{spec}(T)$. O conjunto resolvente de um operador linear é um conjunto aberto pois seu complementar, o espectro, é compacto. O raio espectral do $T$ é definido como $r(T)=\sup \{|x-y| ; x, y \in \operatorname{spec}(T)\}$. O raio espectral tem a seguinte caracterização

$$
r(T)=\liminf _{n}\left\|T^{n}\right\|^{\frac{1}{n}}=\lim _{n \rightarrow \infty}\left\|T^{n}\right\|^{\frac{1}{n}} .
$$

Também é conhecido que $\operatorname{spec}(T) \subset B(0, r(T))$ e que $\operatorname{spec}(T)=\operatorname{spec}\left(T^{*}\right)$, onde $T^{*}: X^{*} \rightarrow X^{*}$ denota o adjunto de $T$. 
Definição B.3.1. Seja $T: X \rightarrow X$ um operador linear limitado e $\gamma$ uma curva de Jordan retificavel contida em $\rho(T)$, então definimos a proejeção espectral $\pi_{T}: X \rightarrow X$ do seguinte modo

$$
\pi_{T}=\frac{1}{2 \pi} \int_{\gamma}(\lambda I-T)^{-1} d \lambda .
$$

Observação B.3.2. Se o interior de $\gamma$ esta contido no interior de $\rho(T)$ então $\pi_{T}=0$. Por outro lado se $\operatorname{spec}(T)$ está contido inteiramente no interiror de $\gamma$ então $\pi_{L}=I d$.

Proposição B.3.3. Seja $T: X \rightarrow X$ é um operador linear limitado. Então $\pi_{T}$ é uma projeção, i.e, $\pi_{T}^{2}=\pi_{T}$. Alémdo mais $\pi_{T}$ comuta com $T$.

Um subconjunto $\operatorname{spec}(T)$ o qual é simultaneamente aberto e fechado em $\operatorname{spec}(T)$ é chamado conjunto espectral. Seja $\Sigma(T) \subset \operatorname{spec}(T)$ um conjunto espectral, e $\gamma$ uma curva de Jordan retificávle contida em $\rho(T)$ contendo $\Sigma(T)$ em seu interior. Denote por $\pi_{T, \Sigma(T)}$ a projeção espectral associada a $T$ e $\gamma$, i.e.,

$$
\pi_{T, \Sigma(T)}=\frac{1}{2 \pi} \int_{\gamma}(\lambda I-T)^{-1} d \lambda,
$$

onde $\gamma$ é uma curva de Jordan retificável qualquer envolvendo o conjunto espectral $\Sigma(T)$, completamente contida em $\rho(T)$ e tal que qualquer outro ponto do espectro está fora de $\gamma$.

Denotamos $X_{\Sigma(T)}=\pi_{T, \Sigma(T)} X$ e $T_{\Sigma(T)}=\left.T\right|_{X_{\Sigma(T)}}$.

Proposição B.3.4. Seja $\Sigma(T)$ um conjunto espectral de $\operatorname{spec}(T)$, então $\operatorname{spec}\left(T_{\Sigma(T)}\right)=\Sigma(T)$.

Proposição B.3.5. Sejam $\pi_{1}, \pi_{2}: X \rightarrow X$ projeções sobre $X$, então existe $\epsilon>0$ tal que se $\left\|\pi_{1}-\pi_{2}\right\|<\epsilon \pi_{1}$ e $\pi_{2}$ tem o mesmo posto, i.e, $\operatorname{dim} \pi_{1}(X)=\operatorname{dim} \pi_{2}(X)$. 
112 APÊNDICE B. ALGUNS RESUltados ANÁLISE E TEORIA ESPECTRAL 


\section{Referências Bibliográficas}

[1] A. T. Baraviera, L. M. Cioletti, A. O. Lopes, J. Mohr and R. R. Souza. On the general one-dimensional XY Model: positive and zero temperature, selection and non-selection. Rev. Math. Phys., v. 23, p. 1063-1113, 2011.

[2] V. Baladi. Positive Transfer Operators and Decay of Correlations. World Scientific Publishing Co., 2000.

[3] L. BARREIRA. Thermodynamic Formalism and Applications to Dimension Theory. Birkhauser, 2010.

[4] R. Bissacot, E. Garibaldi. Weak KAM methods and ergodic optimal problems for countable Markov shifts. Bull. Braz. Math. Soc., v. 41, p. 321-338, 2010.

[5] T. Bousch. La condition de Walters. Ann. Sci. École Norm. Sup., v. 34 p. 287-311, 2001.

[6] R. Bowen. Equilibrium states and the ergodic theory of Anosov diffeomorphisms. Lecture Notes in Mathematics, v. 470, Springer, 1994.

[7] R. Bowen. Hausdorff dimensions of quasicircles. IHES Publ. Math., v. 50, p. 11-25, 1977.

[8] M. Cassandro And E. Olivieri. Renormalization group and analyticity in one dimension: a proof of Dobrushin's theorem, Comm. Math. Phys., v. 80, p. 255-269, 1981.

[9] L. Cioletti And A. Lopes. Interactions, Specifications, DLR probabilities and the Ruelle Operator in the One-Dimensional Lattice. ArXiv-1404.3232, 2014.

[10] L. Cioletti, M.Denker, A. Lopes, M. Stadlbauer. Spectral Properties of the Ruelle Operator for Product Type Potentials on Shift Spaces. ArXiv-1605.07936, 2016.

[11] L. Cioletti And E. A. Silva. Spectral Properties of the Ruelle Operator on the Walters Class over Compact Spaces, to appear in Nonlinearity, 2017.

[12] R. L. Dobrushin. Analyticity of correlation functions in one-dimensional classical systems with slowly decreasing potentials. Comm. Math. Phys., v. 32, p. 269-289, 1973. 
[13] Y. DaOn. Bernoullicity of equilibrium measures on countable Markov shifts Discrete Contin. Dyn. Syst., v. 33, p. 4003-4015, 2013.

[14] F. J. Dyson. Existence of a phase-transition in a one-dimensional Ising ferromagnet. Comm. Math. Phys., v. 12, p. 91-107, 1969.

[15] R. ElLis Entropy, Large Deviation and Statistical Mechanics. Springer, 2005.

[16] A.H. FAn. A proof of the Ruelle theorem. Reviews Math. Phys., v. 7, p. 1241-1247, 1995.

[17] A. Fan, Y. Jiang. On Ruelle-Perron-Frobenius Operators. I. Ruelle Theorem. Comm. Math. Phys., v. 223, p. 125-141, 2001.

[18] J. FröHLich, T. SPEnCER. The phase transition in the one-dimensional Ising model with $1 / r^{2}$ interaction energy. Comm. Math. Phys. v. 84, p. 87-101, 1982.

[19] J, Glimm And A. JAFFe. Quantum physics: A functional integral point of view. Second edition, Springer-Verlag, New York, 1987.

[20] Braga, G., And Hilario, M.. O Teorema de Perron-Frobenius e a Ausência de Transiçao de Fase em Modelos da Mecânica Estatística. Anais das Jornadas de Iniciação Científica do IMPA, Rio de Janeiro, 2004.

[21] O. Jenkinson. Ergodic optimization, Discrete and Continuous. Dynamical Systems, Series A, v. 15, p. 197-224, 2006.

[22] O. Jenkinson, D. Mauldin And M. URBanski. Ergodic optimization for countable alphabet subshifts of finite type. Erg.Theo. and Dyn. Syst., v. 26, p. 1791-1803, 2006.

[23] Y. P. Jiang. A Proof of existence and simplicity of a maximal eigenvalue for RuellePerron-Frobenius operators. Letters in Math. Phys., v. 48, p. 211-219, 1999.

[24] H.-O. Georgin, Gibbs Measures and Phase Transitions. de Gruyter, Berlin, 1988.

[25] S. GouËzel. Sharp polynomial estimates for the decay of correlations. Israel J. Math., v. 139, p. 29-65, 2004.

[26] J. GiniBre. General formulation of Griffiths' inequalities. Comm. Math. Phys., v. 16, p. 310-328, 1970.

[27] R. B. Griffiths. Correlations in Ising Ferromagnets. I. J. Math. Phys. v. 8, p. 478-483, 1967.

[28] R. B. GRIfFithS. Rigorous results for Ising ferromagnets of arbitrary spin. J. Math. Phys., v. 10, p. 1559-1565, 1969.

[29] G. Keller. Equilibrium states in ergodic theory. London Mathematical Society Student Texts, Vol. 42, Cambridge University Press, 1998. 
[30] D. G. Kelly and S. Sherman. General Griffiths' Inequalities on Correlations in Ising Ferromagnets. J. Math. Phys. v. 9, p. 466-484, 1968.

[31] E. Lima. Espaços Métricos. Terceira Edição, Projeto Euclides, Rio de Janeiro, 2005.

[32] A. O. Lopes, J. Mohr, J. Mengue, And R. R. Souza. Entropy and Variational Principle for one-dimensional Lattice Systems with a general a-priori measure: finite and zero temperature. Erg. Theo. and Dyn. Syst., v. 35, p. 1925-1961, 2015.

[33] E. Lorch. Spectral theory. University Texts in the Mathematical Sciences, Oxford University Press, New York, 1962.

[34] R. MAÑÉ. Generic properties and problems of minimizing measures of Lagrangian systems. Nonlinearity, v. 9, p. 273-310, 1996.

[35] R. MAÑÉ. The Hausdorff dimension of horseshoes of diffeomorphisms of surfaces, Bol. Soc. Brasil. Mat., v. 20, p. 1-24, 1990.

[36] A. Manning, H. McCluskey. Hausdorff dimension for horseshoes, Erg. Theo. and Dyn. Syst., v. 3, p. 251-260, 1983.

[37] W. Parry And M. Pollicott. Zeta functions and the periodic orbit structure of hyperbolic dynamics. Astérisque, v. 187-188, 1990.

[38] Y. B. Pesin. Dimension theory in dynamical systems contemporary views and application. Chicago Lectures in Mathematics Series, 1997.

[39] D. RuelLe. Statistical mechanics of a one-dimensional lattice gas. Comm. Math. Phys. v. 9, p. 267-278, 1968.

[40] D. RuelLe. Thermodynamic Formalism. Second edition, Cambridge, 2004.

[41] O. SARIG . Thermodynamic formalism for countable Markov Shifts. Erg.Theo. and Dyn. Syst., v. 19, p. 1565-1593, 1999.

[42] O. SARIG. Subexponential decay of correlations. Invent. Math., v. 150, p. 629-653, 2002.

[43] O. SARIG . Lecture Notes on Thermodynamic Formalism for Topological Markov Shifts. Preprint PenState USA, 2009.

[44] E. A. Silva, R. R. Silva And R. R. Souza. The analyticity of a generalized Ruelle operator. Bull. Braz. Math. Soc., v. 45, p. 53-72, 2014.

[45] E. A. Silva. Dimensão de Hausdorff de Ferraduras. Universidade de Brasília Departamento de Matemática - Monografia de Mestrado, 2010.

[46] F. Spitzer. A Variational characterization of finite Markov chains. The Annals of Mathematical Statistics. v. 43, p. 303-307, 1972.

[47] M. VianA. Stochastic dynamics of deterministic systems. Notes IMPA, v. 21, 1997. 
[48] P. Walters. Invariant Measures and Equilibrium States for Some Mappings which Expand Distances. Trans. Amer. Math. Soc., v. 236, p. 121-153, 1978.

[49] P. WALters. An introduction to ergodic theory. Graduate Texts in Mathematics, Vol. 7, Springer-Verlag, New York-Berlin, 1982.

[50] P. WALters. A natural space of functions for the Ruelle operator theorem. Erg. Theo. and Dyn. Syst., v. 27, p. 1323-1348, 2007. 